Aus der Klinik für Allgemein-, Viszeral- und Kinderchirurgie

(Univ.-Prof. Dr. med. B. M. Ghadimi)

der Medizinischen Fakultät der Universitätsmedizin Göttingen

\title{
Oxaliplatin in der perioperativen, multimodalen Behandlung (präoperative Chemoradiotherapie, TME-Chirurgie und postoperative Chemotherapie) des Rektumkarzinoms - eine monozentrische Analyse -
}

\author{
INAUGURAL-DISSERTATION \\ zur Erlangung des Doktorgrades \\ der Medizinischen Fakultät der \\ Georg-August-Universität zu Göttingen \\ vorgelegt von \\ Beate Michels, geb. Skubich \\ aus \\ Bruchköbel
}

Göttingen 2020 
Dekan:

\section{Betreuungsausschuss}

Betreuer/in:

Ko-Betreuer/in:

\section{Prüfungskommission}

Referent/in:

Ko-Referent/in:

Drittreferent/in:
Prof. Dr. med. W. Brück

Prof. Dr. med. T. Liersch

PD Dr. med. F. Braulke

Prof. Dr. med. T. Liersch

PD Dr. med. F. Braulke

Prof. Dr. med. T. Meyer

Datum der mündlichen Prüfung: 11.02.2021 
Hiermit erkläre ich, die Dissertation mit dem Titel

„Oxaliplatin in der perioperativen, multimodalen Behandlung (präoperative Chemoradiotherapie, TME-Chirurgie und postoperative Chemotherapie) des Rektumkarzinoms - eine monozentrische Analyse -“

eigenständig angefertigt und keine anderen als die von mir angegebenen Quellen und Hilfsmittel verwendet zu haben.

Göttingen, den

(Unterschrift) 


\section{Publikationsliste}

Daten aus der vorgelegten Dissertation wurden bereits teilweise publiziert:

Quack H, Erpenbeck L, Wolff HA, Sprenger T, Seitz CS, Schön MP, Neumann S, Stanek K, B. Michael Ghadimi BM, Michels B, Middel P, Schaefer IM, Liersch T, Conradi LC (2013): Oxaliplatin-induced leukocytoclastic vasculitis under adjuvant chemotherapy for colorectal cancer: Two cases of a rare adverse event, Case Reports in Oncology, 2013 Dec 11, 6, 609-15 


\section{Inhaltsverzeichnis}

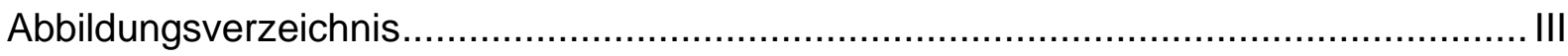

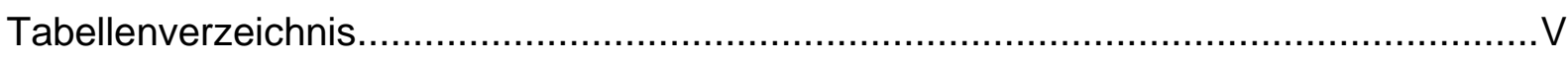

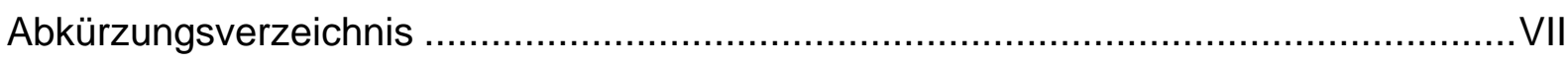

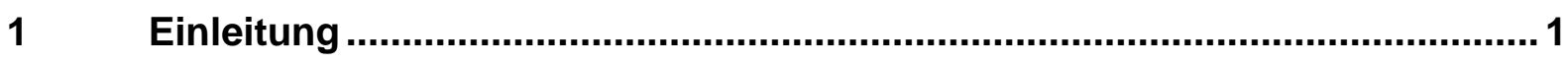

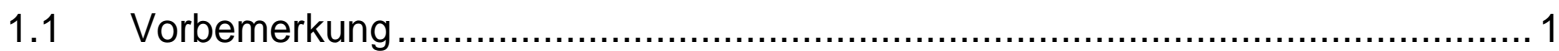

1.2 Kolorektale Karzinome - Inzidenz und Mortalität........................................ 2

1.3 Kolorektale Karzinome - Screeningmaßnahmen........................................... 3

1.4 Kolorektale Karzinome - Staging und Therapieprinzipien .............................. 5

1.5 Kolonkarzinome - chirurgische Maßnahmen .......................................... 8

1.6 Rektumkarzinome - multimodale Therapiemaßnahmen................................. 12

1.7 Nachsorge bei Kolon- und Rektumkarzinomen............................................ 13

1.8 Herleitung der aktuellen Fragestellung ................................................... 13

1.9 Zielsetzung und Fragestellung......................................................... 16

$2 \quad$ Material, Methoden und Patienten................................................................... 17

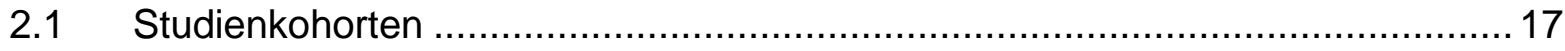

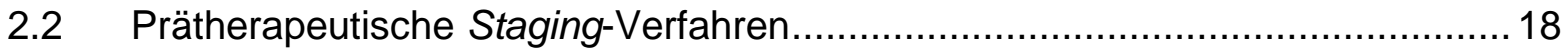

2.3 Perioperative multimodale Behandlung .................................................. 18

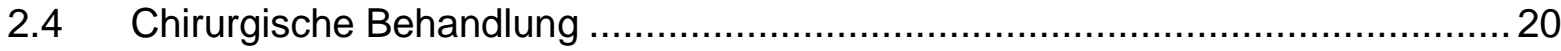

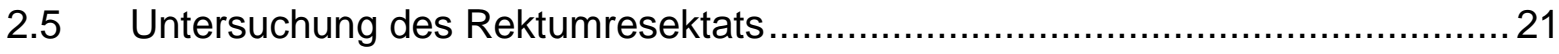

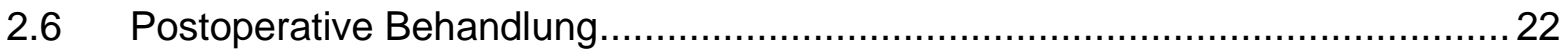

$2.7 \quad$ Chemotherapie - eingesetzte Substanzen ................................................. 23

2.8 RT-/CTx-Dosisdichte sowie auftretende Nebenwirkungen................................ 37

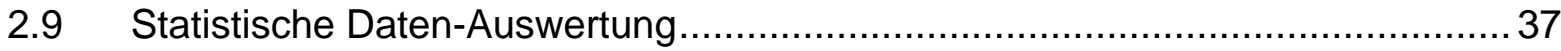

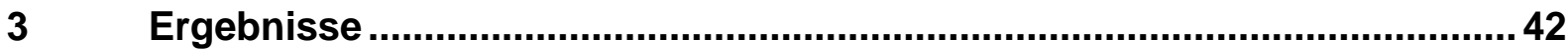

3.1 Präoperative MMT - Dosisapplikation und Toxizität ................................... 44

3.2 Operationsverfahren und TME-Qualität ................................................ 44

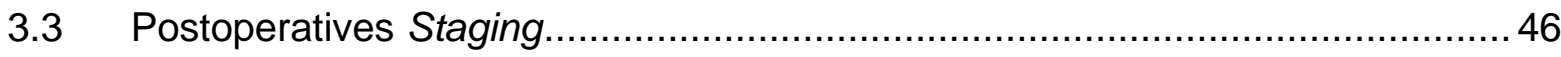


3.4 Präoperative Multimodaltherapie - Toxizitätsgrade .................................. 49

3.5 Postoperative additive CTx, Dosis und Toxizitäten ...................................... 54

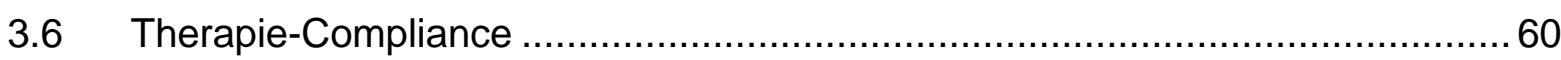

3.7 Nachsorge - Lokalrezidive und Fernmetastasen ..................................... 62

3.8 Nachsorge - Überlebenszeiten und Lebensalter ........................................ 64

3.9 Überleben nach perioperativer Multimodaltherapie ..................................... 65

3.10 Univariate Survivalanalysen zu den ypUICC-Stadien .................................. 67

3.11 Univariate Survivalanalyse zum Tumoransprechen .................................... 73

3.12 Univariate Survivalanalyse zum ypL-/V-/Pn-Status ................................. 77

3.13 Weitere Parameter nach MMT mit Einfluss auf das Überleben......................... 82

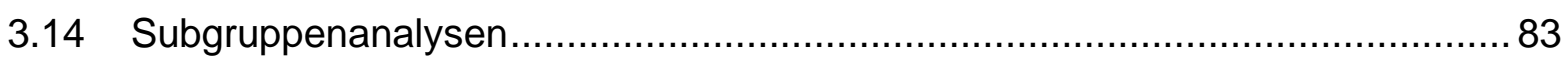

3.15 Multivariate Analysen - Überleben - Einfluss von Oxaliplatin.......................... 88

4 Diskussion

4.1 Allgemeiner Stellenwert der adCTx beim Rektumkarzinom ..........................94

4.2 Ergebnisse im Kontext zu publizierten klinischen Studien ........................... 109

Schlussfolgerung und Ausblick ......................................................... 114

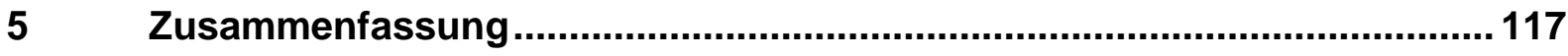

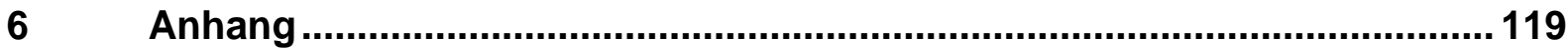

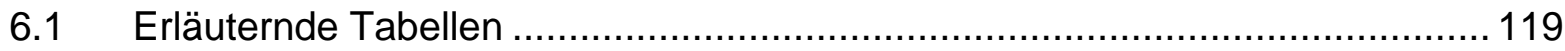

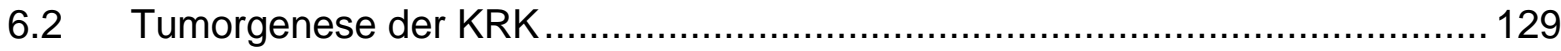

6.3 Erbliche Pathogenese und weitere Risikofaktoren der KRK ......................... 130

6.4 KRK - Karzinome unterschiedlicher Organsysteme ................................... 131

6.5 Tumor-Klassifikation - Basissystematik............................................... 133

6.6 Prinzipien der MMT und Chirurgie beim Rektumkarzinom ........................... 137

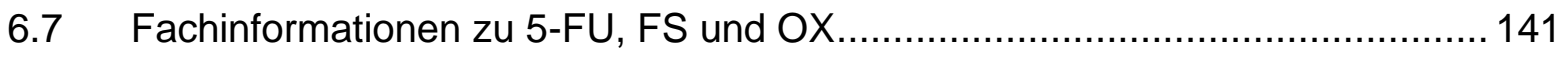

6.8 Therapiepläne der Kohorten A, B und C ............................................... 142

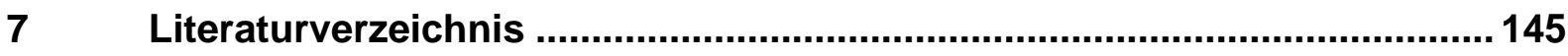




\section{Abbildungsverzeichnis}

Abbildung 1: Inzidenz der absoluten und jährlichen Neuerkrankungen für KRK (ICD-10: C18-C21) in Deutschland nach Altersgruppen (Zeitraum

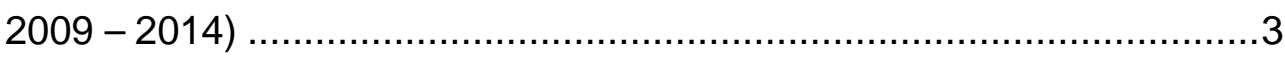

Abbildung 2: Perioperative Multimodaltherapie - Patientenkohorten A bis C ...........20

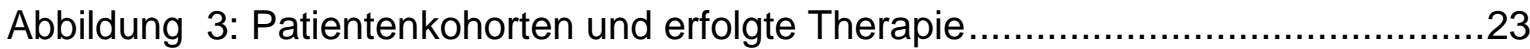

Abbildung 4: Wirkmechanismen von 5-FU und Oxaliplatin (OX) bei KRK ................24

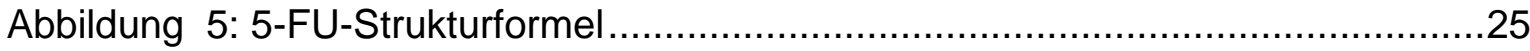

Abbildung 6: Strukturformel der Folinsäure .......................................................

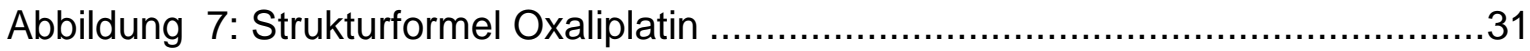

Abbildung 8: Oxaliplatin-Addukte und OX-bedingte DNA-Konformationsänderung ...32

Abbildung 9: Applikation der additiven CTx via Port-System ambulant auf der IKO des G-CCC der UMG 34

Abbildung 10: Leukozytoklastische Vaskulitis als CTx-Nebenwirkung........................36

Abbildung 11: Leukozytoklastische Vaskulitis - Immunhistochemie/-fluoreszenz ......36

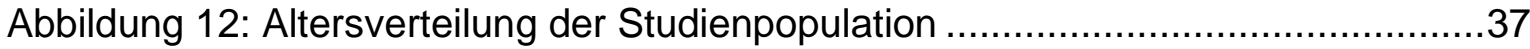

Abbildung 13: Studienpopulation und Auswertungsparameter (I) nach MMT ............66

Abbildung 14: DFS in Abhängigkeit von den ypUICC-Stadien 0-IV ...........................68

Abbildung 15: RFS in Abhängigkeit von den ypUICC-Stadien 0-IV ..........................69

Abbildung 16: CSS in Abhängigkeit von den ypUICC-Stadien 0-IV ..........................71

Abbildung 17: OS in Abhängigkeit von den ypUICC-Stadien 0-IV ............................73

Abbildung 18: DFS in Abhängigkeit zum Tumoransprechen (gut vs. partiell) .............74

Abbildung 19: CSS in Abhängigkeit zum Tumoransprechen (gut vs. partiell) .............75

Abbildung 20: OS in Abhängigkeit zum Tumoransprechen (gut vs. partiell) ...............77

Abbildung 21: DFS in Abhängigkeit zum ypL-/V-/Pn-Status ....................................78

Abbildung 22: CSS in Abhängigkeit zum ypL-/V-/Pn-Status .................................... 80

Abbildung 23: OS in Abhängigkeit des ypL-/V-/Pn-Status ........................................81

Abbildung 24: Studienpopulation und Auswertungsparameter (II) nach MMT ...........83

Abbildung 25: K-M-Überlebenskurven (I) nach Ansprechen auf die MMT ..................86

Abbildung 26: K-M-Überlebenskurven (II) nach Ansprechen auf die MMT ................86

Abbildung 27: RFS und CSS nach Tumoransprechen auf die MMT .........................87

Abbildung 28: DFS und OS nach Tumoransprechen auf die MMT ..........................88 
Abbildung 29: Multivariate Analyse (Forest-Plot-Darstellung) zum CSS. .89

Abbildung 30: CSS aller Patienten mit abgeschlossener perioperative MMT und

Eintritt in die Nachsorgephase .91

Abbildung 31: CSS in Korrelation zu den ypUICC-Stadien und Patienten-Kohorten ..93 Abbildung 32: DFS in Abhängigkeit zu kompletten Applikationen/Zyklen der adCTx .98 Abbildung 33: OS in Abhängigkeit zu kompletten Applikationen/Zyklen der adCTx ...99 Anhang

Abbildung A1: Adenom-Karzinom-Sequenz 129

Abbildung A2: Therapieplan der Kohorten A, B und C für präoperative CTx 143 Abbildung A3: Therapiepläne der Kohorten A und B für postoperative CTx, Kohorte C präoperative CTx nach CRT 144 


\section{Tabellenverzeichnis}

Tabelle 1: Kolon- und Rektumkarzinom-Screening in Deutschland und Österreich....4

Tabelle 2: Einteilung der Kolon- und Rektumkarzinome nach Lokalisation ................5

Tabelle 3: Ausbreitungsdiagnostik bei Kolon- und Rektumkarzinomen ......................7

Tabelle 4: Chirurgische Maßnahmen bei Kolonkarzinomen ....................................8

Tabelle 5: KRK-Stadien nach TNM-/UICC-Klassifikation und Überleben ...................9

Tabelle 6: Postoperative Maßnahmen beim Kolonkarzinom nach pUICC-Stadien ...10

Tabelle 7: Tumorstadien-adaptierte adCTx bei Kolonkarzinomen ...........................11

Tabelle 8: Multimodale Therapiemaßnahmen nach S3-Leitlinie KRK ......................12

Tabelle 9: Überlebensparameter nach Konsensusvereinbarungen .........................38

Tabelle 10: Überlebensparameter - Ereignisse und Endpunkte ..............................39

Tabelle 11: Patientenkohorten und auswertbare CRF-Dokumentation .......................40

Tabelle 12: Prätherapeutisches Staging und Re-Staging ....................................43

Tabelle 13: Operationsverfahren und Beurteilung der TME-Qualität ..........................45

Tabelle 14: Pathologisches Staging und Tumor-Klassifikation ................................47

Tabelle 15: Residuelle Tumorlängsausdehnung (ypTE) und Tumorregression ..........48

Tabelle 16: Akute Nebenwirkungen präoperativer MMT (Kohorte A) .........................50

Tabelle 17: Akute Nebenwirkungen präoperativer MMT (Kohorte B) .........................51

Tabelle 18: Akute Nebenwirkungen unter präoperativer MMT (Kohorte C) ................52

Tabelle 19: Akute Nebenwirkungen präoperativer mFOLFOX-CTx (Kohorte C).........53

Tabelle 20: Postoperative Therapie in den Patientenkohorten A und B.....................56

Tabelle 21: Akute Nebenwirkungen unter adCTx in Kohorte A................................58

Tabelle 22: Akute Nebenwirkungen unter adCTx in Kohorte B................................59

Tabelle 23: Therapie-assoziierte Nebenwirkungen und Therapie-Compliance ...........61

Tabelle 24: Auftreten von Lokalrezidiven (LR) und Fernmetastasen (FM) ..................62

Tabelle 25: Überlebenszeit nach Altersgruppen (Quartile) .....................................64

Tabelle 26: Überlebenszeit nach Clusterung des Lebensalters in Dekaden ................65

Tabelle 27: DFS (rMW) in Abhängigkeit von den ypUICC-Stadien 0-IV ....................67

Tabelle 28: RFS (rMW) in Abhängigkeit von den ypUICC-Stadien 0-IV ....................69

Tabelle 29: CSS (rMW) in Abhängigkeit von den ypUICC-Stadien 0-IV .....................71

Tabelle 30: OS (rMW) in Abhängigkeit von den ypUICC-Stadien 0-IV ......................72

Tabelle 31: DFS (rMW) in Abhängigkeit zum Tumoransprechen (gut vs. partiell) ......74 
Tabelle 32: CSS (rMW) in Abhängigkeit zum Tumoransprechen (gut vs. partiell) ......75

Tabelle 33: OS (rMW) in Abhängigkeit zum Tumoransprechen (gut vs. partiell) ........76

Tabelle 34: DFS (rMW) der Patienten in Abhängigkeit zum ypL-/V-/Pn-Status ..........78

Tabelle 35: CSS der Patienten in Abhängigkeit zum ypL-/V-/Pn-Status .....................79

Tabelle 36: OS (rMW) der Patienten in Abhängigkeit zum ypL-/V-/Pn-Status ............81

Tabelle 37: Überleben (rMW) in Abhängigkeit zum ypTE- und ypCRM-Status...........82

Tabelle 38: Ansprechen gruppiert nach ypUICC- und sekundär nach TRG-Status ....84

Tabelle 39: Überleben nach modifizierter Einteilung des Tumoransprechens .............85

Tabelle 40: Daten der Therapiewechsler, die FOLFOX als adCTx erhielten ..............92

Tabelle 41: DFS (rMW) und OS (rMW) der Patienten in Abhängigkeit zu

Applikationen/Zyklen adCTx

Anhang

Tabelle A 1: Patienten ohne additive Chemotherapie (adCTx)

Tabelle A 2: Patienten der Kohorten A und B mit Dosisreduktionen in der präoperativen CRT

Tabelle A 3: Patienten Kohorte C mit Dosisreduktion in der präoperativen MMT ....121

Tabelle A 4: Patienten der Kohorten A und B mit Dosisreduktion der adCTx ..........125

Tabelle A 5: Akute Nebenwirkungen unter präoperativer MMT (Kohorten A, B, C) .126

Tabelle A 6: Akute Nebenwirkungen unter adCTx (Kohorten A und B) ..................127

Tabelle A 7: Residual-, TRG-Status, Rezidive, 3-Jahre DFS und OS nach Alter.....128

Tabelle A 8: Risikofaktoren für das Auftreten von Kolon- und Rektumkarzinomen..131

Tabelle A 9: Basissystematik der TNM-Klassifikation .........................................133

Tabelle A10: Einteilung der KRK nach 8. Auflage der TNM-/UICC-Klassifikation .....134

Tabelle A11: TNM-/UICC-Stadieneinteilung z. B. beim Rektumkarzinom.................135

Tabelle A12: Residual(R)-Klassifikation ...................................................... 136

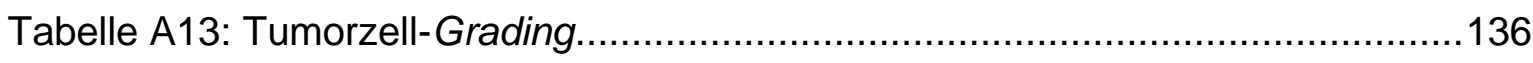

Tabelle A14: Tumor-Ansprechen auf eine präoperative Multimodaltherapie gruppiert nach TRG 


\section{Abkürzungsverzeichnis}

AIO

AJCC

APC

APR

ARO

ARR

ARDS

Al

B-RAF

BRAF

C

CI

CAO

CME

CR

CRM

CRO

CRT

CSS

CT

CTC

CTx

DACH

DFS

DGHO

DNA

DPD

DRKS

ECOG
Arbeitsgemeinschaft Internistische Onkologie

American Joint Committee of Cancer (TNM-/UICC-Klassifikation)

Adenomatous-polyposis-coli-Protein/-Gen

abdomino-perineale Rektumresektion

Arbeitsgemeinschaft Radiologische Onkologie

anteriore Rektumresektion

acute respiratory distress syndrome, akutes Atemnotsyndrom

Anastomoseninsuffizienz

rapidly acceleration fibrosarcoma oder rat fibrosarcoma, RAF-

Proteinkinase/Isoform B-RAF

BRAF-Proto-Onkogen zu B-RAF

clinical, klinisch

confidence interval, Konfidenzintervall

Chirurgische Arbeitsgemeinschaft Onkologie

complete mesocolic excision, komplette mesokolische Exzision

complete remission, komplette Remission

circumferential resection margin, zirkumferentieller Resektionsrand

Clinical Research Organisation, Organisation für klinische Forschung kombinierte Chemo-/Radiotherapie

cancer-specific survival, krebsspezifisches Überleben

Computertomographie

Common Toxicity Criteria, Allgemeine Toxizitätskriterien

Chemotherapie

1,2-Diaminocyclohexal-Ligand

disease-free survival, krankheitsfreies Überleben

Deutsche Gesellschaft für Hämatologie und Medizinische Onkologie desoxyribonucleic acid (DNS), Desoxyribonukleinsäure

Dihydropyrimidindehydrogenase

Deutsches Register Klinische Studien

Eastern Cooperative Oncology Group; ECOG/WHO Score: vereinfachter ECOG-Performance-Status mit 6 Punkten 
EGFR epidermal growth factor receptor, epidermaler WachstumsfaktorRezeptor

EMVI extramural vascular invasion, extramurale Gefäßinfiltration

ESMO European Society for Medical Oncology

EORTC European Organisation for Research and Treatment of Cancer

ETS extramural tumor spread, extramurale Tumorausbreitung

FdUMP Fluorodesoxyuridinmonophosphat

FdUTP Fluorodesoxyuridintriphosphat

FM Fernmetastasen

FOLFIRI CTx-Regime mit Folinsäure, Fluorouracil (5-FU) und Irinotecan

FOLFOX CTX-Regime mit Folinsäure, Fluorouracil (5-FU) und Oxaliplatin

FA/ FS folinic acid, Folinsäure

\section{5-FU 5-Fluorouracil}

G grading, Differenzierung von Karzinomzellen

GCP good clinical practice, gute klinische Praxis

GR good response, gutes Ansprechen des Tumors auf eine Therapie

Gy Gray, Einheit der Energiedosis

HIPEC Hypertherme intraperitoneale Chemotherapie

HNPCC hereditary nonpolyposis colorectal cancer, herditäre nicht-Polyposisassoziertes kolorektales Karzinom

HR hazard ratio, Risiko für ein Ereignis

ICD International Statistical Classification of Diseases and related health

IQR interquartile range, Interquartilsabstand

KFO Klinische Forschergruppe

KRAS KRAS-Gen/-Mutation, ein Proto-Onkogen im Kirsten RAt Sarcoma-

Virus erstmals identifiziert, K-RAS-Protein (G-Protein)

KM Kontrastmittel

K-M Kaplan-Meier

KRK Kolorektale Karzinome

L Lymphgefäße

LARC locally advanced rectal cancer, lokal fortgeschrittenes Rektumkarzinom in den Tumorstadien UICC-II und -III

LE Lungenembolie 
LFU Ioss to follow-up, Kontaktabbruch zum Patienten in der Nachsorge

LM Lebermetastasen

LMP Iamina muscularis propria

LK/LKM Lymphknoten/Lymphknotenmetastase

LR Lokalrezidiv

M Metastasen

moAk monoklonaler Antikörper

MLH MutL-Homolog 1-Gen, MLH1, 2 und 6

MMR mismatch repair system, DNA-Mismatch-Reparaturproteine-/Gene

MMT Multimodaltherapie

MRI/MRT magnetic-resonance-imaging/Magnetresonanztomographie

MSI Mikrosatelliten-Instabilität

N Nodalstatus, Lymphknoten-(LK-)status

$\mathbf{N C l} \quad$ National Cancer Institut

NCT National Cancer Trial

nCR near complete response, nahezu komplette Tumorremission

OS overall survival, Gesamtüberleben

OX Oxaliplatin

p

PCR

pathologisch, in Kombination mit dem TNM-Klassifikationssystem

pCR

polymerase chain reaction, Polymerase-Kettenreaktion

pathologisch bestätigte complete remission, komplette Remission

PFS progression-free survival, progressionsfreies Überleben

p53 Tumorsuppressor-Protein p53

PME partial mesorectal excision, partielle mesorektale Exzision

Pn Perineuralscheideninfiltration

pT (histo-)pathologisch bestimmter Tumorstatus

PR partial response, teilweises Ansprechen

R (histo-)pathologisch bestimmter postoperativer Residualstatus

RAS Rat Sarcoma, kleines G-Protein (monomer) codierendes Protoonkogen

RFS relapse-free survival, rezidivfreies Überleben

rMW restriktiver Mittelwert (auch: restricted mean survival time (RMST), beschränkte erwartete mittlere Überlebenszeit) 
RNA ribonucleic acid, Ribonukleinsäure

rES rektale Endosonographie

RT Radiotherapie

SSL Steinschnittlage

SAPV Spezialisierte ambulante Palliativversorgung

T Tumor, Tumorgröße/Tumorinfiltrationstiefe

TARR tiefe anteriore Rektumresektion

TE tumor extent, Tumorausdehnung

TDM therapeutisches Drugmonitoring

TME total mesorectal excision, totale mesorektale Exzision

TEM transanale endoskopische Mikrochirurgie

TNM Klassifikationssystem maligner Tumore: $\mathbf{T}=$ Tumor, Tumorgrö-

Be/Tumorinfiltrationstiefe, $\mathbf{N}=$ Nodalstatus, $\mathbf{M}=$ Metastasen

TP Thymidinphosphorylase

TRG tumor regression grading, Tumorregressionsgrad

TS Thymidilatsynthase

u endosonographische Einschätzung, in Kombination mit dem TNM-

Klassifikationssystem (uT)

UICC Union Internationale Contre le Cancer, Internationale Vereinigung gegen Krebs

UTP Uridintriphosphat

V Venengefäße

VEGF vascular endothelial growth factor, vaskulärer endothelialer Wachstumsfaktor

WHO World Health Organization, Weltgesundheitsorganisation

WHS Wundheilungsstörung

y Status nach präoperativer Therapie, in Kombination mit dem TNM-

Klassifikationssystem 


\section{$1 \quad$ Einleitung}

\section{$1.1 \quad$ Vorbemerkung}

In dieser Dissertation wird der Einfluss des Zytostatikums Oxaliplatin (OX) während der perioperativen Multimodaltherapie (MMT) von Patienten mit Rektumkarzinomen dargestellt. Alle Patienten wurden prospektiv unter den Bedingungen klinischer Phase-II- und Phase-III-Studien mit einer präoperativen Chemo-/Radiotherapie (CRT) +/- Chemotherapie (CTx) in der Universitätsmedizin der GeorgAugust-Universität Göttingen (UMG) behandelt. Anschließend wurden die Patienten einer qualitätskontrollierten totalen mesorektalen Exzision (TME) und einer postoperativen additiven CTx (adCTx) und/oder Nachbeobachtung zugeführt.

Die Datenauswertung umfasst 177 Patienten, die zwischen 07/2006 und 06/2016 in der Klinik für Allgemein- und Viszeralchirurgie (bis 03/2012 Direktor: Univ.-Prof. Dr. med. H. Becker, in memoriam; seit 04/2012 Direktor: Univ.-Prof. Dr. med. B. M. Ghadimi) der UMG eine TME +/- adCTx erhielten. Anschließend wurden die Patienten über $>5$ Jahre in Kooperation mit den Zuweisern/Hausärzten über die Klinik für Allgemein-, Viszeral- und Kinderchirurgie (CALL) und das CALLStudiensekretariat nachbeobachtet.

Es bestanden Kooperationen mit dem Institut für Diagnostische und Interventionelle Radiologie (Direktor: Univ.-Prof. Dr. med. J. Lotz), der Klinik für Strahlentherapie und Radioonkologie (Direktor: Univ.-Prof. Dr. med. Dr. rer. nat. C.-F. Hess), dem Institut für Pathologie (Direktoren: Univ.-Prof. Dr. med. H.-J. Radzun (bis 06/2018), Univ.-Prof. Dr. med. Ph. Ströbel), der Klinik für Hämatologie und Medizinische Onkologie (Direktor: Univ.-Prof. Dr. med. L. Trümper) und der InterdisziplinärenKurzzeit-Onkologie (IKO) des Göttinger Comprehensive Cancer Centers (G-CCC).

Im nachfolgenden Text wird auf eine genderspezifische Schreibweise verzichtet. Alle Patient*innen-bezogenen Angaben sind genderneutral zu verstehen. 


\subsection{Kolorektale Karzinome - Inzidenz und Mortalität}

Krebserkrankungen des Kolons (ICD-10: C18), des Rektosigmoids (ICD-10: C19), des Rektums (ICD-10: C20) und des Anus (ICD-10: C21) werden im medizinischen Sprachgebrauch unter dem Begriff Kolorektale Karzinome (KRK) unabhängig von ihrer Kodierung im International Classification of Disease (ICD)-10 System zusammengefasst (DIMDI 2019, Bray et al. 2018, Steliarova-Foucher et al. 2015). Im Jahr 2018 sind 58.047 KRK-Neuerkrankungen und 27.334 KRK-bedingte Todesfälle für Deutschland angegeben worden (IARC 2018a). Damit stehen KRK bei den Krebserkrankungen an dritter Stelle und bei krebsbedingten Todesfällen an zweiter Stelle. International gehört Deutschland zu den 10 Ländern mit den meisten KRK-Neuerkrankungen und Todesfällen (IARC 2018b).

In Abbildung 1 werden die Neuerkrankungsraten für KRK in Deutschland gezeigt. Derzeit erkranken Männer früher, d. h. zwischen dem 50. und 79. Lebensjahr, und häufiger an KRK als Frauen. Das mittlere Erkrankungsalter der Männer liegt bei 70,3 Jahren, und die Altersgruppe der 70- bis 74-Jährigen ist am häufigsten betroffen (Kaatsch et al. 2015). Aufgrund ihrer genderspezifischen Lebenserwartung sinkt bei den > 80-jährigen Männern die Rate an KRK im Vergleich zu den Frauen ab. Derzeit liegt das mittlere Erkrankungsalter für Frauen bei 72,9 Jahren (RKI 2016).

KRK (ICD: C18-C21) sind aber nicht nur als Alterskrebserkrankungen anzusehen. Aus epidemiologischen Studien lässt sich eine zunehmende Inzidenz der KRK in der Bevölkerung der < 65-Jährigen herleiten. Insbesondere Männer erkranken ab dem 50. Lebensjahr, und eine im berufsfähigen Alter diagnostizierte KRKErkrankung stellt für den Patienten eine starke Herausforderung dar. Neben ihrer unmittelbaren Behandlungsbedürftigkeit führt die KRK-Erkrankung häufig zu erheblichen sozioökonomischen Belastungen für den Betroffenen, seine Familie und die Gesellschaft (Brenner et al. 2016; 2014a; 2014b; Luengo-Fernandez et al. 2013). 


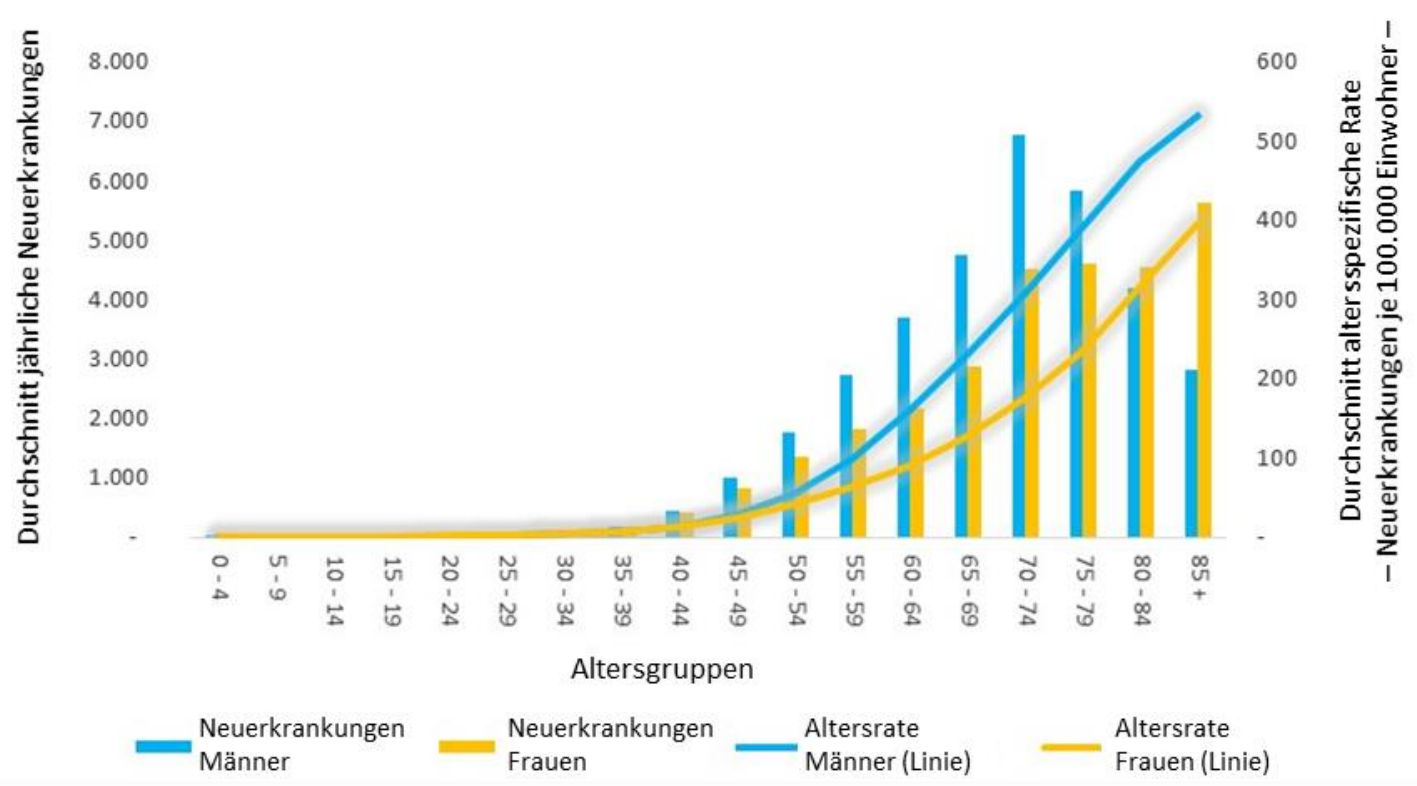

Abbildung 1: Inzidenz der absoluten und jährlichen Neuerkrankungen für KRK (ICD-10: C18-C21) in Deutschland nach Altersgruppen (Zeitraum 2009 - 2014)

KRK: kolorektale Karzinome; Balken und linke Y-Achse: Angabe der jährlichen Zahl an Neuerkrankungen; Linien und rechte Y-Achse: altersspezifische Rate an KRK; selbsterstellte Abbildung unter Nutzung von Datenbankabfragen beim Zentrum für Krebsregisterdaten des Robert-Koch-Instituts Berlin. Der dargestellte Zeitraum beginnt mit dem Jahr 2009, in dem erstmalig die statistische Erfassung aller Bundesländer zur Verfügung gestanden hatte.

Für Deutschland wird derzeit eine Rate von $12 \%$ bis $24 \%$ an KRK-Neuerkrankungen prognostiziert. Dieser Anstieg an KRK ergibt sich allein aus den Veränderungen der Bevölkerungsstruktur (DGHO 2019, Statistisches Bundesamt (Destatis) 2019).

\subsection{Kolorektale Karzinome - Screeningmaßnahmen}

Vor 25 Jahren wurden in Deutschland Programme zur Darmkrebs-Früherkennung für die Bevölkerung der <60-Jährigen eingeführt. Diese Programme umfassten eine rektal-digitale Untersuchung, den Nachweistest auf okkultes Blut in der Stuhlprobe, eine starre Rektosigmoidoskopie und die Koloskopie mit einem flexiblen Endoskop. Mittlerweile wird die Koloskopie als "Goldstandard" für alle krankenversicherten Bürger ab dem 55. Lebensjahr empfohlen. Die Kosten für die Vorsorgeuntersuchungen werden bei gesetzlich Krankenversicherten durch die Krankenkassen übernommen (Brenner et al. 2017; Krebsfrüherkennungsrichtlinie 2002). 
Im Vergleich zu einigen europäischen Nachbarländern, wie z. B. Österreich, setzen die KRK-Vorsorgeuntersuchungen in Deutschland später ein (Tabelle 1).

\begin{tabular}{|c|c|c|c|}
\hline \multicolumn{4}{|c|}{ Darmkrebs-Screening } \\
\hline Intervall & Maßnahme & Deutschland & Österreich \\
\hline \multirow[b]{2}{*}{ jährlich } & $\begin{array}{l}\text { digitale rektale } \\
\text { Untersuchung }\end{array}$ & ab dem 50. Lebensjahr & ab dem 40. Lebensjahr \\
\hline & $\begin{array}{l}\text { Test auf okkultes Blut im } \\
\text { Stuhl (immunchemisch, } \\
\text { iFOBT) }\end{array}$ & 50. bis 54. Lebensjahr & ab dem 40. Lebensjahr \\
\hline \multirow[b]{2}{*}{ > jährlich } & $\begin{array}{l}\text { Test auf okkultes Blut im } \\
\text { Stuhl (immunchemisch, } \\
\text { iFOBT) }\end{array}$ & $\begin{array}{l}\text { zweijährlich ab dem } 55 \text {.Lebens- } \\
\text { jahr als Alternative zur Kolosko- } \\
\text { pie }\end{array}$ & \\
\hline & komplette Koloskopie* & $\begin{array}{l}\text { Männer ab dem } 50 . \text { Lebens- } \\
\text { jahr }{ }^{\star \star} \text {, Frauen ab dem } 55 . \text { Le- } \\
\text { bensjahr; Wiederholung nach } \\
10 \text { Jahren bei unauffälligem } \\
\text { Befund* }\end{array}$ & $\begin{array}{l}\text { ab dem } 50 \text {. Lebensjahr; } \\
\text { alle } 5 \text { bis } 7 \text { Jahre }\end{array}$ \\
\hline
\end{tabular}

Tabelle 1: Kolon- und Rektumkarzinom-Screening in Deutschland und Österreich

Eigene tabellarische Darstellung; * abhängig vom Befund wird eine Wiederholung der Koloskopie durch einen erfahrenen ärztlichen Erstuntersucher festgelegt; ${ }^{* *}$ gemäß Beschluss des Gemeinsamen Bundesausschusses über die Richtlinie für organisierte Krebsfrüherkennungsprogramme und eine Änderung der Krebsfrüherkennungs-Richtlinie vom 19.07.2018; (BMG 2018).

Ob eine Herabsetzung des Screeningalters für Männer auf das 50. Lebensjahr auch in Deutschland zu dem gewünschten Ergebnis einer Senkung fortgeschrittener Tumorstadien bei Erstdiagnose führen wird, bleibt aufgrund der Ergebnisse der GEDA-2014/2015-EHIS-Studie abzuwarten (Starker et al. 2018). In dieser Studie des Robert-Koch-Instituts Berlin wurde bei 9.489 Personen (4.878 Frauen, 4.611 Männer) der Zeitpunkt der letztmaligen Koloskopie seit dem 55. Lebensjahr abgefragt. Nur $57 \%$ der Frauen und 61 \% der Männer gaben an, dass bei innen eine Koloskopie innerhalb der letzten 10 Jahre durchgeführt worden sei. In der Altersgruppe der 55- bis 59-Jährigen lag die Koloskopierate sogar < $50 \%$. Diese geringe Inanspruchnahme von Vorsorgemaßnahmen lässt befürchten, dass ausgedehntere Screening-Angebote von der Bevölkerung in Deutschland nicht angenommen werden. 


\subsection{Kolorektale Karzinome - Staging und Therapieprinzipien}

Klinisch beginnt die Zuordnung eines histologisch gesicherten Tumors zu Kolonoder Rektumkarzinomen bereits während der initialen Ausbreitungsdiagnostik (Staging). Nach der aktuellen S3-Leitlinie Kolorektales Karzinom (2019) hat neben der Koloskopie immer eine starre Rektosigmoidoskopie zu erfolgen. Diese ermöglicht eine endoluminale Positionsangabe des aboralen Tumorunterrands und erlaubt die Festlegung, ob die Tumorformation dem Rektum (bis $16 \mathrm{~cm}$ ab Anokutanlinie, ACL) oder dem Kolon (> $16 \mathrm{~cm}$ ab anocutan) zugerechnet werden muss (Tabelle 2). Ist die Tumorformation im Kolon, so erfolgt die Lokalisationsangabe während des Rückzugs des flexiblen Koloskops (in gestrecktem Zustand) aus dem Darm. Zuvor sollte die Tumorlokalisation mit Hilfe von Tusche oder Titan-Clips markiert worden sein. Während der Biopsie werden idealerweise mehrere $>5 \mathrm{~mm}$ messende Tumorproben einschließlich unauffälliger Darmmukosa entnommen und für diagnostische sowie molekular-genetische Analysen asserviert.

\begin{tabular}{|l|l|l|l|}
\hline \multicolumn{2}{|c|}{} & \multicolumn{1}{|c|}{ UICC-Klassifikation } & \multicolumn{1}{c|}{ ESMO-Klassifikation } \\
\hline & Tumor-Lokalisation & ab linea anocutanea (la) & ab linea anocutanea (la) \\
\hline Kolonkarzinom & gesamtes Kolon & $>16 \mathrm{~cm}$ & $>15 \mathrm{~cm}$ \\
\hline \multirow{4}{*}{ Rektumkarzinom } & oberes Rektumdrittel & $>12 \mathrm{~cm}$ bis $16 \mathrm{~cm}$ & $>10 \mathrm{~cm}$ bis $15 \mathrm{~cm}$ \\
\cline { 2 - 5 } & mittleres Drittel & $>6 \mathrm{~cm}$ bis $12 \mathrm{~cm}$ & $>5 \mathrm{~cm}$ bis $10 \mathrm{~cm}$ \\
\cline { 2 - 4 } & unteres Drittel & $\leq 6 \mathrm{~cm}$ & $\leq 5 \mathrm{~cm}$ \\
\hline
\end{tabular}

Tabelle 2: Einteilung der Kolon- und Rektumkarzinome nach Lokalisation

Eigene tabellarische Darstellung; die Distanzangabe zwischen der Anokutanlinie und dem Tumorunterrand erfolgt während der Rektoskopie (in $\mathrm{cm}$ ). Die Tumorlage wird üblicherweise nach der TNM-/UICC- (Wittekind und Meyer 2010) und weniger nach der ESMO-Klassifikation festgelegt (Schmoll et al. 2012).

Beim Rektumkarzinom (ICD-10: C20) erfolgt zusätzlich eine Zuordnung der Tumoren zum unteren ( $\leq 6 \mathrm{~cm}$ ab $A C L)$, mittleren ( $>6 \mathrm{~cm}$ bis $12 \mathrm{~cm}$ ab $A C L)$ und oberen Rektumdrittel (> $12 \mathrm{~cm}$ bis $16 \mathrm{~cm}$ ab ACL) (Wittekind und Meyer 2010). Die in Tabelle 2 sichtbare Diskrepanz zwischen der TNM-/UICC- und ESMOKlassifikation in der Höhenzuordnung von Rektumkarzinomen kann bei der Effizienzbewertung moderner Therapieverfahren von Relevanz sein. So können unterschiedliche Zuordnungen die Aussage zur Wirksamkeit einer präoperativen 
CRT z. B. bei Karzinomen im oberen Rektumdrittel verfälschen, wenn ein Großteil der Tumoren eigentlich dem mittleren Rektumdrittel hätte zugerechnet werden müssen (Tabelle 2). In dieser Dissertation wird ausschließlich die Einteilung der UICC-/TNM-Klassifikation des American Joint Committee of Cancer (AJCC, TNM-) und der Union Internationale Contre le Cancer (UICC) (Classification of Malignant Tumours; Sobin et al. 2011) verwendet (Anhang 6.5).

Neben den Befunden aus der Ausbreitungsdiagnostik (Tabelle 3) sind die histopathologischen Ergebnisse aus den Tumorbiopsaten oder nach Polypabtragung die Grundlage für eine weitere Therapie. Diese richtet sich nach dem klinischen Krankheitsstadium (cUICC-Stadium) und Risikofaktoren, wie z. B. der Tumorinfiltrationstiefe bei Frühkarzinomen (T1), der Perineuralscheiden- (Pn1), Lymphgefäß- (L1) und Blutgefäßinfiltration (V1) bei den die Lamina muscularis propria (LMP) durchbrechenden, fortgeschrittenen Tumoren (T3) sowie dem Nachweis weiterer molekulargenetischer Prognoseparameter. Dazu gehören Faktoren des Mismatch-Repair(MMR)-Systems, die Mikro-Satelliten-Instabilität (MSI) als Ausdruck einer fehlerhaften DNA-Reparatur mit Veränderungen in der repetitiven DNA-Sequenz, Mutationen des BRAF-Gens (z. B. Nachweis einer BRAF-V600EMutation, bei der nach Austausch von Valin gegen Glutaminsäure das Proteinkinase Isomer B-RAF aktiviert wird, es gehört zur Familie der RAF-Proteine: rapidly acceleration fibrosarkoma), KRAS-Mutationen des "Kirsten rat sarcoma viral oncogens" (z. B. KRAS-Mutationen in Codon 12 und 13 des Exons 2 oder 3 durch DNA-Sequenzierung) und Non-RAS-Mutationen (RAS-Protoonkogen mit Mutationen im Exon 2, 3 und 4). Sie gehören zur RAS(rat sarcoma)-Proto-OnkogenFamilie (Gains und Whiting 2002). K-RAS ist ein kleines G-Protein (molekular Gewicht von $21 \mathrm{kDa}$ ), das eine wichtige Rolle in zellulären Signaltransduktionsprozessen spielt. Eine therapeutische Konsequenz kann die Mutationsdetektion in der Therapie kolorektaler Karzinome mit Anti-EGFR-Antikörpern haben (Stintzing et al. 2009) (Abschnitt 1.5.1; 1.6; 2.7.3.1 und 6.3). 


\begin{tabular}{|c|c|c|c|}
\hline \multirow[b]{2}{*}{ Methode } & \multicolumn{2}{|c|}{ Ensatz beim } & \multirow[b]{2}{*}{ Befunderhebung } \\
\hline & \begin{tabular}{|l|} 
Kolon- \\
karzinom
\end{tabular} & $\begin{array}{l}\text { Rektum- } \\
\text { karzinom }\end{array}$ & \\
\hline \multirow[t]{2}{*}{ rektal-digital } & \multirow[t]{2}{*}{$\mathrm{x}$} & \multirow[t]{2}{*}{$\mathrm{x}$} & $\begin{array}{l}\text { Prüfung der Sphinkterfunktion und des Kneifdrucks; Beurteilung des Analkanals sow ie der } \\
\text { Schleimhaut im unteren Rektumdrittel, der Stuhlfarbe und -konsistenz, Kontrolle einer Bei- } \\
\text { mengung frischen Blutes als Zeichen einer aktiven Blutung }\end{array}$ \\
\hline & & & \begin{tabular}{|l|} 
Positionsangabe des Tumors in SSL, Mason-Kriterien (Mason 1976a; 1976b) mit digitaler \\
Überprüfung der Verschieblichkeit des Tumors gegenüber der Rektumw and \\
\end{tabular} \\
\hline \multirow{4}{*}{ starre Rektoskopie } & \multirow{4}{*}{$\mathrm{x}$} & \multirow{4}{*}{$\mathrm{X}$} & $\begin{array}{l}\text { intraluminale Bestimmung der transversalen TU-Ausdehnung im Uhrzeigersinn und in Stein- } \\
\text { Schnitt-Lage (SSL-Position) des Patienten; Angabe der longitudinalen Tumorausdehnung, } \\
\text { sofern dieser mit dem Rektoskop überw indbar ist }\end{array}$ \\
\hline & & & $\begin{array}{l}\text { Messung des Abstands (in cm) zw ischen aboralem Tumorunterrand und der Anokutanlinie } \\
\text { sow ie Angabe der Distanz zur Linea dentata }\end{array}$ \\
\hline & & & mehrfache Biopsie (jew eils > 5 mm) von Tumorgew ebe und unauffälliger Mukosa \\
\hline & & & Markierung der Tumorlage vor RT, z. B. per (Gold-) Clipmarkierung (Vorw erk et al. 2009) \\
\hline \multirow[t]{2}{*}{$\begin{array}{l}\text { rektale } \\
\text { Endosonographie } \\
\text { (rES) }\end{array}$} & \multirow[t]{2}{*}{-} & \multirow[t]{2}{*}{$\mathrm{X}$} & \begin{tabular}{|l|} 
dynamische intraluminale Untersuchung zur TU-Infiltration in die Rektumwandschichten \\
analog des TNM-/UICC-Systems; uT1- und uT2-Tumoren sind limitierte Befunde; bei \\
Durchbruch durch die Lamina muscularis propria (LMP) liegt ein uT3-Status vor, Angabe \\
der intra-mesorektalen Tiefeninfiltration in mm und Klassifikation in uT3a- bis uT3d-Status \\
Tumor-Ausbreitung in Nachbarorgane/-strukturen (UT4-Tumor); eine derartige Beurteilung \\
per rES ist häufig nur eingeschränkt möglich
\end{tabular} \\
\hline & & & \begin{tabular}{|l|} 
Beurteilung der intramesorektalen Lymphknoten (LK); bei LK > $5 \mathrm{~mm}$ und einem suspekten \\
Binnenecho besteht der V.a. auf LK-Metastasen (UN+ Status) (Liersch et al. 2003; Merkel et \\
al. 2001); insgesamt sind die Sensitiviät und Spezifität der rES in der Beurteilung von LK- \\
Metastasen analog des TNM-/UICC-Systems unzureichend (Puli et al. 2009)
\end{tabular} \\
\hline \multirow{3}{*}{ Koloskopie } & \multirow{3}{*}{$\mathrm{x}$} & \multirow{3}{*}{$\mathrm{x}$} & \begin{tabular}{|l|} 
endoskopische Kontrolle des gesamten Kolonrahmens mit Markierung der Tumorlokalisation \\
nach Biopsie; die Tumorlage bestimmt das onkologisch notw endige OP-Ausmaß
\end{tabular} \\
\hline & & & Ausschluss von Zw eitkarzinomen (5\%-15\%) (Liersch et al. 2005; Barillari et al. 1990) \\
\hline & & & \begin{tabular}{|l|} 
bei einer partiellen Koloskopie aufgrund einer tumorbedingten Passagebehinderung erfolgt \\
die Restkoloskopie intraoperativ oder 3-6 Monaten postoperativ; in Ausnahmefällen ist eine \\
CT-gestützte virtuelle Koloskopie indiziert (Neri et al. 2002)
\end{tabular} \\
\hline \multirow{5}{*}{ pelvines MRT } & \multirow{5}{*}{ - } & \multirow{5}{*}{$\mathrm{X}$} & 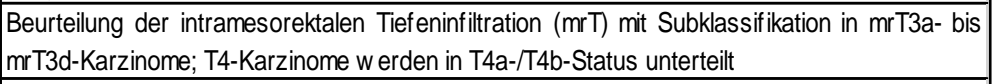 \\
\hline & & & \begin{tabular}{|l|l|} 
Beurteilung des LK- Status (mrN-Status) mit Subklassen (mrNO-, mrN1- und mrN2-Status) \\
\end{tabular} \\
\hline & & & \begin{tabular}{|l|} 
Beurteilung des zirkumferentiellen Resektionsrandes (CRM-positiv: < 2 mm tumorfreier \\
Randsaum) (Kim et al. 2009; 2006; Kw ok et al. 2000) \\
\end{tabular} \\
\hline & & & Bestimmung der cranio-caudalen (longitudinalen) Tumorausdehnung (TE) \\
\hline & & & \begin{tabular}{|l|} 
Bestimmung prognostischer Parameter wie z. B. der extramuralen vaskulären Tumorinfiltra- \\
tion (EMVI) und der Therapie-induzierten Tumorregression (mrTRG)
\end{tabular} \\
\hline Leber-MRT & $(\mathrm{X})$ & $(\mathrm{X})$ & \begin{tabular}{|l|} 
Leber-MRT (individuelle Indikationsstellung) bei V.a. Lebermetastasierung nach vorausge- \\
gangener Kontrastmittel-Sonographie und nach CT-Diagnostik (Niekel et al. 2010)
\end{tabular} \\
\hline $\begin{array}{l}\text { Abdomen } \\
\text { Sonographie }\end{array}$ & $\mathrm{x}$ & $\mathrm{x}$ & $\begin{array}{l}\text { Abdomensonographie zur "real-time“ Detektion von suspekten Raumforderungen (z. B. } \\
\text { Lebermetastasen) mit hoher Sensitivität ( } 86 \% \text { ) und Spezifität (98\%); bei V. a. LM wird der } \\
\text { Ensatz der Kontrastmittel-gestützten (KM-) Sonographie empfohlen (Floriani et al. 2010) }\end{array}$ \\
\hline Röntgen Thorax & $\mathrm{x}$ & $X$ & \begin{tabular}{|l|} 
Darstellung des Lungenparenchyms, Ausschluss größerer Lungenmetastasen (> $5 \mathrm{~mm}) ;$ \\
Kontrolle von Veränderungen der Lungenstruktur
\end{tabular} \\
\hline $\begin{array}{l}\text { CT } \\
\text { Thorax/Abdomen }\end{array}$ & $x$ & $X$ & $\begin{array}{l}\text { CT-Thorax: folgt der Röntgen-Thorax-Untersuchung bei suspekten intrathorakalen } \\
\text { Raumforderungen/Veränderungen; CT-Abdomen: Nachw eis intrahepatischer und/oder } \\
\text { intraperitonealer und/oder lymphatischer Fernmetastasen; orientierende Beurteilung von } \\
\text { ossären Läsionen (ggf. Enleitung weiterer Spezialdiagnostik); Beurteilung der pelvinen } \\
\text { Organe }\end{array}$ \\
\hline $\begin{array}{l}\text { Tumormarker } \\
\text { (CEA und CA19-9) }\end{array}$ & $X$ & $X$ & $\begin{array}{l}\text { CEA ist der einzige Tumormarker mit prognostischer Relevanz; er wird sinnvollerw eise mit } \\
\text { CA19-9 kombiniert (Duffy et al. 2001); TU-Marker-Bestimmungen erfolgen prä- und } \\
\text { postoperativ sowie im Verlauf der Nachsorge (zu definierten Zeitpunkten) }\end{array}$ \\
\hline
\end{tabular}

Tabelle 3: Ausbreitungsdiagnostik bei Kolon- und Rektumkarzinomen

Tabellarische Zusammenstellung auf der Grundlage der S3-Leitlinie Kolorektales Karzinom (2019) 


\subsection{Kolonkarzinome - chirurgische Maßnahmen}

Die nationale S3-Leitlinie Kolorektales Karzinom wurde erstmals 1998 veröffentlicht und in den Folgejahren mehrfach überarbeitet. Nach der aktuellen S3-Leitlinie (2019) werden die folgenden chirurgischen Maßnahmen empfohlen (Tabelle 4).

\begin{tabular}{|l|l|}
\hline Tumore und Dignität & \multicolumn{1}{|c|}{ Chirurgische Maßnahmen nach S3-Leitlinie KRK } \\
\hline Kolontumore unklarer oder günstiger & $\begin{array}{l}\text { endoskopische Submukosadissektion (ESD) zur histologischen Sicherung; } \\
\text { lokale Abtragung von Kolonpolypen (low-risk Tumor; pT1, G1 / G2, pL0), } \\
\text { sofern keine Hohlorganperforation zu erwarten ist }\end{array}$ \\
\hline Kolonkarziome mit ungünstiger Dignität & $\begin{array}{l}\text { onkologische Kolonresektion für high-risk Polypen und Frühkarzinome } \\
\text { (pT1, pG3 u./o. pL1/V1) (Jass und Sobin 1989) sowie T1-T4 } \\
\text { Kolonkarzinome bzw. cUICC-Stadien I-IIl; die Resektion orientiert sich an } \\
\text { der Tumorlokalisation, der Gefäßversorgung und am Lymphabfluß des } \\
\text { betroffenen Kolonabschnitts und dessen zentraler Gefäßversorgung aus } \\
\text { der Aorta abdominalis. Zur Vermeidung einer Tumorzelldissemination } \\
\text { werden bei der OP primär die radikuläre Gefäßdissektion, das no-touch- } \\
\text { Prinzip des tumortragenden Darmsegments, das Legen von Turnbull- } \\
\text { Ligaturen zur Vermeidung einer intraluminalen Tumorzellfreisetzung und } \\
\text { eine en-bloc Resektion von tumoradhärenten Nachbarstrukturen unter } \\
\text { Einhaltung eines Sicherheitsabstandes durchgeführt. Zudem sind das } \\
\text { geschlossene Absetzen des tumortragenden Darmabschnitts mit } \\
\text { Klammernahtgeräten, das in toto Bergen des Resektats sowie eine } \\
\text { Spülung des Situs mit physiologischer Kochsalzlösung wesentliche OP- } \\
\text { Schritte (Becker und Liersch 2006). }\end{array}$ \\
\hline
\end{tabular}

Tabelle 4: Chirurgische Maßnahmen bei Kolonkarzinomen

Unter Einhaltung dieser Resektionsprinzipien und unter Vermeidung onkologisch unzureichender Verfahren, wie z. B. der Transversumresektion mit Ascendodescendostomie bei Karzinomen im Colon transversum, konnten in den vergangenen 20 Jahren sowohl das rezidivfreie Überleben (recurrence-free survival, RFS) wie auch das Gesamtüberleben (overall survival, OS) der Patienten verbessert werden (André et al. 2009). Mit Einführung der kompletten mesokolischen Exzision (CME), d. h. der en-bloc Mitnahme retropankreatischer LymphknotenAreale bei flexurennahen Karzinomen im Colon ascendens ergab sich für die Hemikolektomie mit lleotransversostomie eine $10 \%$-ige Verbesserung der 5-Jahre OS-Rate (Bertelsen et al. 2015; Hohenberger et al. 2009).

Die genannten Resektionsprinzipien gelten unabhängig davon, ob die OP offen per medianer Laparotomie oder laparoskopisch bzw. Roboter-unterstützt (z. B. mit einem DaVinciß-System) durchgeführt wird (Chen et al. 2018; Brenner et al. 2017; Babaei et al. 2016; Grade et al. 2016). 


\subsubsection{Kolonkarzinome - postoperative Prognoseabschätzung}

Zur Prognoseabschätzung und vor Einleitung einer Chemotherapie ist die histopathologische Gesamtbeurteilung des Primärtumors, des Gradings (z. B. G2), des Residualstatus (z. B. R0-Status, Anhang 6.5.2), der OP-Qualität (z. B. gute Qualität der CME) und die Untersuchung von > 12 LK im Resektat erforderlich (Renzulli et al. 2006; Renzulli und Laffer 2005) (Tabelle 5).

\begin{tabular}{|c|c|c|c|c|c|c|}
\hline \multirow{2}{*}{$\begin{array}{l}\text { UICC- } \\
\text { Stadium }\end{array}$} & \multicolumn{3}{|c|}{ TNM Klassifikation } & \multirow{2}{*}{ T-/N-/M-Befund } & \multicolumn{2}{|c|}{$\begin{array}{l}5 \text { Jahre Über- } \\
\text { lebensrate (\%) }\end{array}$} \\
\hline & $\begin{array}{l}\text { Tumor- } \\
\text { Status }\end{array}$ & $\begin{array}{l}\text { Nodal- } \\
\text { Status }\end{array}$ & $\begin{array}{l}\text { FM- } \\
\text { Status }\end{array}$ & & Kolon & Rektum \\
\hline 0 & Tis & \multirow{5}{*}{ NO } & \multirow{13}{*}{ MO } & Carcinoma in situ/intramukosales Karzinom & \multicolumn{2}{|c|}{100} \\
\hline 1 & $\mathrm{~T} 1, \mathrm{~T} 2$ & & & $\begin{array}{l}\text { Tumor beschränkt sich auf Mukosa und Submukosa } \\
\text { (T1), infiltriert maximal in die LMP (T2) }\end{array}$ & \multicolumn{2}{|c|}{$>95$} \\
\hline lla & T3 & & & $\begin{array}{l}\text { LMP-Durchbruch, Infiltration in die Subserosa oder in } \\
\text { nicht peritonealisiertes perikolisches oder perirekta- } \\
\text { les/mesorektales Gewebe }\end{array}$ & \multicolumn{2}{|c|}{$>90$} \\
\hline llb & T4a & & & TU perforiert viszerales/peritoneales Gewebe & $85-90$ & 85 \\
\hline Ilc & $\mathrm{T} 4 \mathrm{~b}$ & & & TU infiltriert angrenzende Organe/Strukturen & 72 & 70 \\
\hline \multirow[t]{2}{*}{ Illa } & T1-2 & N1 & & $\begin{array}{l}\text { N1: 1-3 LKM; N1a: } 1 \text { LKM; N1b: 2-3 LKM; N1c: Satelli- } \\
\text { ten* im Fettgewebe }\end{array}$ & \multirow[t]{2}{*}{83} & \multirow[t]{2}{*}{55,1} \\
\hline & $\mathrm{T} 1$ & $\mathrm{~N} 2 \mathrm{a}$ & & N2a: 4-6 LKM & & \\
\hline \multirow{3}{*}{ Illb } & T3-4 & N1 & & N1: 1-3 LKM & \multirow{3}{*}{64} & \multirow{3}{*}{35} \\
\hline & T2-3 & $\mathrm{N} 2 \mathrm{a}$ & & N2a: 4-6 LKM & & \\
\hline & T1-2 & $\mathrm{N} 2 \mathrm{~b}$ & & N2b: $\geq 7$ LKM & & \\
\hline \multirow{3}{*}{ Illc } & T4a & $\mathrm{N} 2 \mathrm{a}$ & & N2a: 4-6 LKM & \multirow{3}{*}{44} & \multirow{3}{*}{24,5} \\
\hline & T3-T4a & $\mathrm{N} 2 \mathrm{~b}$ & & N2b: $\geq 7$ LKM & & \\
\hline & $\mathrm{T} 4 \mathrm{~b}$ & $\mathrm{~N} 1-2$ & & N1: 1-3-LKM; N2: > 4 LKM & & \\
\hline $\mathrm{IVa}$ & \multirow{3}{*}{ jedes $\mathrm{T}$} & \multirow{3}{*}{ jedes $\mathrm{N}$} & M1a & $\begin{array}{l}\text { FM auf } 1 \text { Organ begrenzt oder }>1 \text { Lokalisation ohne } \\
\text { peritoneale FM }\end{array}$ & \multicolumn{2}{|c|}{$<30$} \\
\hline $\mathrm{IVb}$ & & & M1b & $\begin{array}{l}\text { FM in } \geq 2 \text { Organen oder } \geq 2 \text { Lokalisationen ohne } \\
\text { peritoneale FM }\end{array}$ & \multicolumn{2}{|c|}{$<30$} \\
\hline IVc & & & M1c & $\begin{array}{l}\text { peritonealer Befall }+/ \text { - FM in anderen Organen/Loka- } \\
\text { lisationen }\end{array}$ & \multicolumn{2}{|c|}{ keine Angabe } \\
\hline
\end{tabular}

Tabelle 5: KRK-Stadien nach TNM-/UICC-Klassifikation und Überleben

Eigene tabellarische Zusammenstellung (modifiziert nach Fibbe 2018; Hofheinz et al. 2018a; Wittekind 2017; Wittekind und Meyer 2010, Le Voyer et al. 2003), LKM: Metastasen in Lymphknoten; *Satelliten: makro- oder mikroskopisch erkennbare Tumorzellcluster im Fettgewebe der Subserosa oder im nicht peritonealisierten perikolischen bzw. perirektalen Fettgewebe ohne histologisch erkennbare Reste eines LK

Die Angabe der pUICC-Tumorstadien nach der TNM-/UICC-Klassifikation sowie die klinische Einschätzung des Patienten sind Grundlage für die Indikationsstel- 
lung zur adCTx (Tabelle 7) (Le Voyer et al. 2003). Zudem kann die Kenntnis von molekularbiologischen Parametern, wie z. B. des KRAS-Status, die zur Behandlung eingesetzte Kombination zytostatischer Substanzen mit monoklonalen Antikörpern (moAk) beeinflussen. Diese als „Biologicals“ bezeichneten Medikamente richten sich häufig gegen zelluläre Oberflächenrezeptoren (z. B. gegen EGFRund VEGFR-Bindungsstellen) (Stintzing et al. 2009).

\subsubsection{Kolonkarzinome - postoperative additive Behandlung}

Die nachfolgende Tabelle 6 listet die postoperativen, pUICC-Stadien-adaptierten Behandlungsmaßnahmen bei Kolonkarzinomen auf (Hofheinz et al. 2018a).

\begin{tabular}{|c|c|}
\hline Kolonkarzinom mit pUICC-Stadium & postoperative Maßnahmen \\
\hline pUICC-I und pUICC-Ila & $\begin{array}{l}\text { alleinige Tumornachsorge aufgrund des sehr geringen Rezidiv- } \\
\text { risikos }\end{array}$ \\
\hline pUICC-lllb und pUICC-\|ll & $\begin{array}{l}\text { fakultativ eine adCTx mit 5-FU; trotz eines R0-Status nach } \\
\text { Kolonresektion treten in diesen high-risk Tumorstadien in }<30 \% \\
\text { Rezidive auf. Sie werden als Folge eines pT4-Karzinoms, einer } \\
\text { bereits intraabdominellen gedeckten Tumorperforation, eines } \\
\text { perioperativen iatrogenen Tumoreinrisses oder einer lleus- } \\
\text { bedingten Notfall-OP angesehen (S3-Leitlinie Kolorektales } \\
\text { Karzinom 2019). Zur high-risk Gruppe zählen auch Patienten, bei } \\
\text { denen < 12 LK im Kolonresektat untersucht worden sind, ein } \\
\text { positiver pL-/V-/Pn- oder G3-Status u./o. persistierend hohe CEA- } \\
\text { Spiegel vorliegen. }\end{array}$ \\
\hline pUICC-III & $\begin{array}{l}\text { grundsätzlich eine adCTx; dafür stehen 5-FU-basierte CTx- } \\
\text { Regime zur Verfügung, wie z. B. das AIO-Regime mit 5-FU (c.i.v. } \\
24 \text { h) + Folinsäure (FS), das FOLFOX- (5-FU + FS + OX) oder } \\
\text { das FOLFIRI-Regime (5-FU + FS + Irinotecan). Zudem kann das } \\
\text { oral einzunehmende 5-FU-Prodrug Capecitabin (Xeloda®) allein } \\
\text { oder in Kombination mit OX eingesetzt werden (Tabelle 7). }\end{array}$ \\
\hline pUICC-IV & $\begin{array}{l}\text { üblicherweise eine palliative CTx mit diversen zytotoxischen } \\
\text { Substanzen in Kombination mit moAk (Tabelle 7) }\end{array}$ \\
\hline
\end{tabular}

Tabelle 6: Postoperative Maßnahmen beim Kolonkarzinom nach pUICC-Stadien

Seit Jahrzehnten besteht die Standard-CTx bei KRK aus der Kombination von 5-FU und FS. Dabei verlangsamt die FS den 5-FU-Metabolismus und steigert indirekt die zytostatische Wirkung (Haller et al. 2011; Abbildungen 2 und 4). Durch die Hinzunahme weiterer Zytostatika, wie z. B. von OX, kann die Anti-Tumorwirkung der adCTx erhöht werden. Bei Patienten mit Kolonkarzinomen im Stadium pUICCIII kann ein FOLFOX-Regime das DFS (disease-free survival) und OS (overall survival) um $15 \%$ bis $20 \%$ verbessern (André et al. 2009). 


\begin{tabular}{|c|c|c|c|c|c|c|c|}
\hline \multirow[b]{2}{*}{$\begin{array}{l}\text { pUICC } \\
\text { Status }\end{array}$} & \multicolumn{3}{|c|}{ Postoperatives Staging } & \multirow[b]{2}{*}{ OP } & \multirow[b]{2}{*}{$\begin{array}{c}\text { R- } \\
\text { Status }\end{array}$} & \multirow[b]{2}{*}{$\begin{array}{l}\text { Postop. } \\
\text { Therapie }\end{array}$} & \multirow[b]{2}{*}{ CTx } \\
\hline & \begin{tabular}{|c|} 
pT- \\
Status \\
\end{tabular} & $\begin{array}{c}\mathrm{pN}- \\
\text { Status }\end{array}$ & $\begin{array}{c}\text { FM- } \\
\text { Status }\end{array}$ & & & & \\
\hline I & $\mathrm{pT} 1, \mathrm{pT} 2$ & \multirow{4}{*}{ pNO } & \multirow{6}{*}{ cMO } & \multirow{7}{*}{ CME } & $\mathrm{RO}$ & koino & koino \\
\hline II a & $\mathrm{pT} 3 \mathrm{a} / \mathrm{b}$ & & & & חט & gentre & hentre \\
\hline$\| \mathrm{b}$ & pT3c/d & & & & \multirow{3}{*}{$\mathrm{R} 0, \mathrm{R} 1$} & optional & \multirow{3}{*}{$\begin{array}{l}\text { - FOLFOX } \\
\text { - 5-FU oder Capecitabin } \\
\text { - FOLFIRI }\end{array}$} \\
\hline$\| \mathrm{c}$ & $\mathrm{pT} 4 \mathrm{a} / \mathrm{b}$ & & & & & $\operatorname{adCTx}$ & \\
\hline \multirow{2}{*}{ III a-c } & \multirow{2}{*}{ pT1 - pT4 } & \multirow{2}{*}{$\mathrm{pN} 1 / \mathrm{N} 2$} & & & & $\operatorname{adCTx}$ & \\
\hline & & & & & R2 & & \\
\hline IV a-c & pTx & $\mathrm{pN} 1 / \mathrm{N} 2$ & pM1 & & $\mathrm{R} 1, \mathrm{R} 2$ & $\begin{array}{l}\text { palliative } \\
\mathrm{CTx}, \\
{ }^{\star *} \mathrm{CT} \text { x vor } \\
\text { OP von } \mathrm{FM}\end{array}$ & $\begin{array}{l}\text { - FOLFOXIRI (OX + 5-FU + FS } \\
+ \text { Irinotecan) +/- moAk } \\
\text { - FOLFOX + moAk } \\
\text { - FOLFIRI + moAk } \\
\text { - 5-FU oder Capecitabin +/- moAk }\end{array}$ \\
\hline
\end{tabular}

Tabelle 7: Tumorstadien-adaptierte adCTx bei Kolonkarzinomen

Eigene Darstellung; R0: Residualstatus ohne Tumorrest; R1-Status: mikroskopischer Tumorrest; R2-Status: makroskopischer (R2a) oder mikroskopischer (R2b) Nachweis von in situ verbliebenem Karzinomgewebe; moAk: monoklonale Antikörper, wie z. B. VEGFR-Antikörper: Aflibercept (Zaltrap®), Bevacizumab (Avastin®); oder EGFRAntikörper: Panitumumab (Vectibix®), Cetuximab (Erbitux®); CME: komplette mesokolische Exzision; ${ }^{*}$ CTx: FOLFOXIRI +/- moAk; FOLFOX 6 +/- moAk; FOLFIRI +/- moAk; FM: Fernmetastasen, ggf. sekundäre Resektion der FM nach erfolgter CTx + moAk unter potentiell kurativer Intention möglich 


\subsection{Rektumkarzinome - multimodale Therapiemaßnahmen}

Beim Rektumkarzinom ist die prätherapeutische Einschätzung des cUICCStadiums die wichtigste Voraussetzung für eine erfolgreiche Behandlung. Derzeit wird in Deutschland die in Tabelle 8 aufgeführte Vorgehensweise (S3-Leitlinie Kolorektales Karzinom 2019; Hofheinz et al. 2018b) empfohlen.

\begin{tabular}{|c|c|}
\hline $\begin{array}{l}\text { Rektumkarzinom } \\
\text { cUICC-Stadium }\end{array}$ & Multimodale Therapiemaßnahmen nach S3-Leitlinie KRK \\
\hline $\begin{array}{l}\text { cUICC-I, geringes } \\
\text { Rezidivisiko } \\
\text { (pT1, low-risk) }\end{array}$ & $\begin{array}{l}\text { bei Tumoren }<3 \mathrm{~cm} \text { und G1- oder G2-Status ist eine lokale Vollwandexzision (per TEM) ausreichend, sofern } \\
\text { postoperativ pV0/L0/Pn0- und R0-Status bestätigt werden können }\end{array}$ \\
\hline $\begin{array}{l}\text { cUICC-I, hohes } \\
\text { Rezidivisiko } \\
\text { (pT1, high-risk) }\end{array}$ & $\begin{array}{l}\text { bei Tumoren }<3 \mathrm{~cm} \text { mit G3- oder G4-Differenzierung erfolgt ohne Einsatz einer präoperativen Multimodaltherapie } \\
\text { eine TME bei Karzinomen im unteren (distaler Sicherheitsabstand } \geq 2 \mathrm{~cm} \text { ) und mittleren Rektumdrittel }(\geq 5 \mathrm{~cm} \\
\text { Sicherheitsabstand); bei pT1-high-risk Tumoren im oberen Rektumdrittel ist eine PME (distaler } \\
\text { Sicherheitsabstand } \geq 5 \mathrm{~cm} \text { ) möglich }\end{array}$ \\
\hline \multirow[t]{2}{*}{$\begin{array}{l}\text { cUICC-II } \\
\text { (cT3-cT4 cN0) } \\
\text { und } \\
\text { cUICC-III } \\
\text { (cTx cN1-2) }\end{array}$} & $\begin{array}{l}\text { alleiniger Einsatz einer präoperativen Kurzzeit-RT (Einzeldosis: } 5 \text { Gy/d, Gesamtdosis: } 25 \text { Gy) mit nachfolgender } \\
\text { Therapiepause von 8-12 Wochen oder einer präoperativen } 5 \text {-FU-haltigen CRT (Einzeldosis: } 1,8 \text { Gy bis 2,0 Gy/d, } \\
\text { d 1-d 5, Gesamtdosis: 45,0-50,4 Gy); insbesondere durch die präoperative CRT kann eine Reduktion der } \\
\text { Tumorausdehnung (Tumor-Downsizing) und/oder des UICC-Stadiums (UICC-Downstaging) erreicht werden. } \\
\text { Zudem kann die präoperative CRT bei cT4-Rektumkarzinomen deren Resektabilität (Ziel: Erreichen eines R0- } \\
\text { Status mit einem tumorfreien, zirkumferentiellen Resektionssaum (CRM) von } \geq 2 \mathrm{~mm} \text { ) fördern und zu einer } \\
\text { langanhaltenden Reduktion der Lokalrezidivate führen. Postoperativ komplettiert eine adCTx mit 5-FU diese } \\
\text { multimodale Behandlung; sie ist aufgrund der Langzeitergebnisse der CAO/ARO/AIO-94-Studie in Deutschland } \\
\text { zum Standard für lokal fortgeschrittene Rektumkarzinome des unteren und mittleren Rektumdrittels geworden } \\
\text { (Sauer et al. 2012; 2004) }\end{array}$ \\
\hline & $\begin{array}{l}\text { Eine postoperative CRT (analog des Kontrollarms der CAO/ARO/AIO-94-Studie) bei primär operierten Patienten } \\
\text { mit Rektumkarzinomen der Stadien pUICC-II oder pUICC-III; vor Einführung der präoperativen CRT konnten mit } \\
\text { Hilfe dieser postoperativen RT/CRT die LR-Rate um } 46 \% \text { und die FM-Rate um } 37 \% \text { gesenkt werden mit einer } \\
\text { Verbesserung des krebsspezifischen Überlebens um } 36 \% \text { (Krook et al. 1991); nach postoperativer RT/CRT } \\
\text { erhalten die Patienten eine adCTx mit 5-FU }\end{array}$ \\
\hline \multirow[t]{3}{*}{$\begin{array}{l}\text { cUICC-IV } \\
\text { (cTx, cNx, M1) }\end{array}$} & $\begin{array}{l}\text { multimodale Therapiekonzepte unter Nutzung verschiedener Zytostatika }+/ \text { - moAk; vorab muss allerdings immer } \\
\text { das Ausmaß der Metastasierung bekannt sein }\end{array}$ \\
\hline & $\begin{array}{l}\text { In Fällen mit polytopen, nicht resektablen Leber- und/oder Lungenmetastasen kann eine palliative CTx mit } \\
\text { FOLFOX oder FOLFIRI oder FOLFOXIRI } \pm \text { EGFR/VEGFR-Inhibitoren zu einer Verlängerung des } \\
\text { progressionsfreien Überlebens führen; durch derartige CTX-Regime kann eine Steigerung des OS von } 8 \text { Monaten } \\
\text { auf bis zu } 30 \text { Monate erreicht werden (Kopetz et al. 2009) }\end{array}$ \\
\hline & $\begin{array}{l}\text { unter dem Ziel einer maximal möglichen Lebenszeitverlängerung bei zufriedenstellender Lebensqualität muss, } \\
\text { unter Einbindung eines in der hepatobiliären Chirurgie erfahrenen Viszeralchirurgen, unterschieden werden } \\
\text { zwischen: } \\
\text { - } \quad \text { primär resektablen Leber- und Lungenmetastasen } \\
\text { - sekundär resektablen Leber- und/oder Lungenmetastasen nach systemischer mit CTx +/- moAk oder } \\
\text { CTx +/- Tyrosinkinase-Inhibitoren (TKI) } \\
\text { Einzelfällen mit einer limitierten oder ausgedehnten peritonealen Metastasierung; in letzteren Fällen kann } \\
\text { in Abhängigkeit der Malignomdynamik eine HIPEC-Prozedur mit intraperitonealer CTx erwogen werden } \\
\text { (Verwaal et al. 2008). Die Effektivität der HIPEC, einer Kombination aus ausgedehnter } \\
\text { Multiviszeralresektion (u. a. Peritonektomie) und intra-/perioperativer, in die freie Bauchhöhle applizierter } \\
\text { CTx, ist bisher nur in Fallkohorten-Studien belegt worden. In Deutschland musste } 2018 \text { eine prospektive } \\
\text { Multizenterstudie, die GASTRIPEC-Studie (Rau et al. 2017), aufgrund mangelhafter Patientenrekrutierung } \\
\text { vorzeitig geschlossen werden. }\end{array}$ \\
\hline
\end{tabular}

Tabelle 8: Multimodale Therapiemaßnahmen nach S3-Leitlinie KRK 


\subsection{Nachsorge bei Kolon- und Rektumkarzinomen}

Die Nachsorge KRK erfolgt UICC-Stadien-adaptiert. Nach der S3-Leitlinie Kolorektales Karzinom (2019) wird für das pUICC-I Stadium des Kolonkarzinoms eine Nachsorge nur optional empfohlen.

Für Kolonkarzinome der Stadien pUICC-II/-III,-IV besteht die Nachsorge in den ersten zwei Jahren in halbjährlichen, danach jährlichen Kontrollen bestehend aus einer Anamnese, der körperlichen Untersuchung, einer Abdomen-Sonographie, einem Röntgen-Thorax und der CEA-Bestimmung bis zum 5. Jahr post operationem. Eine Koloskopie sollte nach einem Jahr und nach fünf Jahren durchgeführt werden.

Bei Rektumkarzinomen der Stadien pUICC-II/-III und pUICC-IV nach potentieller R0-Resektion sollte ein CT des Thorax/Abdomens/Beckens drei Monate nach Beendigung der Multimodaltherapie stattfinden. Alle sechs Monate (über mindestens zwei Jahre) sollte eine Rektosigmoidoskopie bei jenen Patienten erfolgen, bei denen zuvor keine präoperative (neoadjuvante) oder postoperative (adjuvante) CRT durchgeführt worden war. Eine jährliche Röntgen-Thorax-Kontrolle wird über mindestens fünf Jahre empfohlen.

Die alleinige endoskopische Kontrolle mit starrer Rektosigmoidoskopie und Koloskopie bilden die Nachsorgemaßnahmen für Rektumkarzinome im pUICC-I Stadium mit R0-Status.

Die o. g. Nachsorge-Empfehlungen gelten auch für alle Tumorstadien (ypUICC-0 bis ypUICC-IV bei R0-Status) nach perioperativer Multimodaltherapie und kurativintendierter Metastasenresektion.

\subsection{Herleitung der aktuellen Fragestellung}

Die präoperative CRT umfasst bei lokal fortgeschrittenen Adenokarzinomen (LARC, locally advanced rectal cancer) des unteren oder mittleren Rektumdrittels die Langzeitbestrahlung (RT: 50,4 Gy) in Kombination mit 5-FU +/- OX gefolgt von einer TME und einer adCTx mit 5-FU +/- OX (Rödel et al. 2015; 2012; Sauer et al. 2012; 2004; García-Aguilar et al. 2003). In einzelnen klinischen Studien ist gezeigt worden, dass die Hinzunahme von OX zur präoperativen CRT zu einem längeren 
RFS, einer Reduktion der LR-Rate und einer stärkeren Tumorregression führen kann (Fokas et al. 2018; 2017; 2015; 2014; Rödel et al. 2015; Martijnse et al. 2012). Dabei reichte der unter Anwendung eines 5-stufigen Tumorregressions$\operatorname{grad}(T R G$ )-Systems (Dworak et al. 1997) ermittelte Therapieeffekt von einer guten (TRG 3 und TRG 4) und partiellen (TRG 1 und TRG 2) Regression bis zur Resistenz (TRG 0). Im Gegensatz zur Wirkung der präoperativen CRT ist die Bedeutung der adCTx ungeklärt. Insbesondere der zusätzliche Effekt von OX wird sehr kontrovers diskutiert (Carvalho und Glynne-Jones 2017).

Auch in der aktuellen Version der S3-Leitlinie Kolorektales Karzinom (2019) wird eine eindeutige Empfehlung zum routinemäßigen Einsatz einer adCTx bei Rektumkarzinom-Patienten nach einer präoperativen CRT und TME vermieden. Diese Zurückhaltung ergibt sich einerseits aus der bekannten Overstaging-Rate von $15 \%$ in der rES zwischen UT2- und UT3-Karzinomen an der Grenzschicht der LMP (Liersch et al. 2003; Mackay et al. 2003; Harewood et al. 2002). Andererseits hat die Begrenzung einzelner eingesetzter Staging-Prozeduren wie z. B. der Verzicht auf die starre Rektoskopie, rES, CT oder pelvine MRT, mit einem Overstaging von $18 \%$ bis $>20 \%$ erheblichen Einfluss auf die Bewertung bisheriger klinischer Studien (Bosset et al. 2014; Sainato et al. 2014; Sauer et al. 2004; BeetsTan und Beets 2004; Beets-Tan et al. 2001).

Unzureichende Definitionen des CRM im Staging und im postoperativen (histo-)pathologischen Befund (Smith et al. 2008) sowie ein heterogenes Therapieinduziertes Karzinomansprechen erschweren zusätzlich die Empfehlung zu einer adCTx. Insbesondere das Ansprechen auf eine präoperative Therapie kann von der kompletten Remission (CR) des Tumorgeschehens (ypT0 N0-Status, ypUICC0 Stadium) in $\leq 15 \%$ der Fälle über eine nahezu CR (ypUICC-I Stadium) in $20 \%$ der Fälle (Maas et al. 2010) bis zu den prognostisch ungünstigen Stadien ypUICCIII und -IV reichen (Chand et al. 2014; Gosens et al. 2007; Mawdsley et al. 2005). Weitere Gründe, die eine allgemeingültige Empfehlung zur adCTx bisher nicht zuließen, liegen in einer in klinischen Studien bisher unzureichenden Qualitätskontrolle der TME (Quirke et al. 2009) und der Resektatoberfläche (wie z. B. bei der APR) als Parameter für die Einhaltung chirurgisch-onkologischer Resektionsprin- 
zipien (Heald 1998; Heald et al. 1998) sowie in einer erheblich eingeschränkten Compliance $(43 \%$ bis $58 \%$ ) der Studienteilnehmer gegenüber der geplanten postoperativen CTx (Mari et al. 2019; Xu et al. 2017; Chand et al. 2014; Wolf et al. 2012; Bosset et al. 2006). Zudem wird immer wieder das unterschiedliche mediane Alter zwischen den Teilnehmern an klinischen Studien gegenüber den NichtTeilnehmern (real-life patients) mit Auswirkungen auf das OS als Argument gegen eine allgemeingültige Empfehlung zur adCTx angeführt (Elliot et al. 2016). 


\subsection{Zielsetzung und Fragestellung}

Das Ziel der vorliegenden Dissertation besteht darin, den Stellenwert von OX in der perioperativen Multimodaltherapie (MMT) bei 177 LARC-Patienten darzustellen. Alle Patienten dieser monozentrischen Auswertung waren im Rahmen prospektiver klinischer Studien standardisierten Staging-Verfahren, einer präoperativen CRT (+/- CTx), einer qualitätskontrollierten TME mit nachfolgender extensiver (histo-)pathologischen Resektataufarbeitung, einer adCTx und/oder einer alleinigen Nachbeobachtung (> 5 Jahre) zugeführt worden.

Folgende Aspekte werden bearbeitet:

1. Beschreibung der Studienpopulation bestehend aus Kohorte A (präoperative $\mathrm{CRT}+\mathrm{TME}+$ adCTx mit 5-FU), Kohorte B (präoperative CRT+OX + TME + adCTx mit 5-FU + OX) und Kohorte C (präoperative CRT + 5-FU + OX und 3x mFOLFOX + TME)

2. Darstellung tumorspezifischer Parameter

3. Darstellung der adCTx (Anzahl applizierter Therapiezyklen, dokumentierte Toxizitätsgrade und Patienten-Compliance); Vergleich mit den Ergebnissen aus der präoperativen Behandlungsphase

4. Beurteilung eines frühen Behandlungserfolgs (z. B. anhand der CRTinduzierten Tumorregression, des UICC-Downstagings sowie weiterer Parameter des Ansprechens auf die MMT)

5. Einfluss der Therapie-induzierten Tumorremission auf das Überleben (DFS, RFS, CSS und OS) und Evaluation von möglichen Prognose-Parametern, wie z. B. des Tumor(T)-Status, der longitudinalen Tumorausdehnung (TE), einer lymphatischen (L1-Status) und/oder venösen Gefäßbeteiligung (V1-Status) und/oder Perineuralscheideninfiltration (Pn1-Status), der individuellen Tumorregression (TRG) und des UICC-Status nach MMT

6. Versuch einer altersadjustierten Analyse der Therapie- und Überlebensergebnisse

7. Vergleich der eigenen Daten mit der Literatur und Schlussfolgerungen. 


\section{Material, Methoden und Patienten}

Nach dem Staging mit klinischer Einschätzung des Tumorstatus ( $\geq$ CUICC-II Stadien) waren alle Patienten dem interdisziplinären Tumorboard des G-CCC der UMG vorgestellt worden. Sämtliche Befunde waren prospektiv dokumentiert, durch eine externe CRO (Clinical Research Organisation) evaluiert und nochmals intern auf Validität überprüft worden. Der weitere Therapieablauf innerhalb der PatientenKohorten $A$ bis $C$ ist in Abbildung 2 übersichtartig dargestellt. Während der MMT angewandte klinische (z. B. die Erhebung von Toxizitäten nach der Common Terminology Criteria for Adverse Events of the National Cancer Institute (CTCAE, Version 4.03, gültig seit 14.06.2010) ( $\mathrm{NCl} 2010$ ) sowie pathologische Einteilungssysteme (TNM-/UICC-Klassifikation) wurden fortlaufend aktualisiert und sind in der zuletzt gültigen Version angegeben.

\subsection{Studienkohorten}

Insgesamt wurden 177 LARC-Patienten mit einem WHO-Performance-Status $E C O G \leq 2$ und einem histologisch nachgewiesenen Adenokarzinom der unteren beiden Rektumdrittel (von 0 bis $12 \mathrm{~cm}$ ab Anokutanlinie) ausgewertet. Vor Erweiterung der Ausbreitungsdiagnostik und vor Einleitung therapeutischer Maßnahmen, einschließlich studienbegleitender Untersuchungen, wurde die schriftliche Einverständniserklärung aller Patienten nach Aufklärung über die vorliegende Erkrankung, die geplanten Therapie-, Nachsorge- und Analyseverfahren eingeholt.

Die perioperative MMT wurde jeweils nach den Vorgaben der CAO/ARO/AIO-04(Rödel et al. 2015; 2012) oder der TransValid-KFO-179/GRCSG-B-Phase-I/-IIStudie (WHO-UTN-U1111-1132-0235) der German Rectal Cancer Study Group (GRCSG) durchgeführt. Die Datenerfassung war prospektiv im Teilprojekt 5aKFO-179-2 (Projektleiter: Prof. Dr. med. T. Liersch; „Prädiktive und prognostische Biomarker beim lokal fortgeschrittenen Rektumkarzinom“; Ethikvotum der Medizinischen Fakultät Göttingen mit Antragsnummer 9/8/08) der Klinischen Forschergruppe 179 begonnen und fortgesetzt worden (KFO-179; Sprecher: Prof. Dr. med. 
B. M. Ghadimi; gefördert durch die Deutsche Forschungsgemeinschaft, DFG). Für beide Studien liegen positive Ethikvoten vor (für die CAO/ARO/AIO-04-Studie, EudraCT-Nr.: 2006-002385-20, ein zentrales Ethikvotum der Medizinischen Fakultät Erlangen-Nürnberg (Bearbeitung-Nr.: 07_2006); und für die TransValid-KFO179/GRCSG-B-Studie, EudraCT-Nr.: 2011-004228-37, ein zentrales Ethikvotum der Medizinischen Fakultät Göttingen (Antrag-Nr.: 23/10/11)). Die Studien und die vorliegenden Analysen wurden nach ICH-GCP (E6) Leitlinie und gemäß der Erklärung von Helsinki (1964) durchgeführt (Wiesing und Parsa-Parsi 2009).

\subsection{Prätherapeutische Staging-Verfahren}

Das prätherapeutische Staging bestand aus einer rektal-digitalen Untersuchung, der starren Rektosigmoidoskopie mit Tumorbiopsien, einer rES und einer pelvinen MRT. Zudem erfolgten Kontrastmittel gestützte CT-Scans von Thorax, Abdomen und Becken zum Ausschluss von FM. Sämtliche Staging-Ergebnisse wurden nach Kontrolle unter oberärztlicher Supervision anhand der TNM-/UICC-Klassifikation als cUICC-Stadium zusammengefasst (Amin et al. 2017; Sobin et al. 2011; Greene et al. 2002). Dabei wurden Rektumkarzinome als cT4-Tumoren angesehen, sofern die Beckenboden- (Ebene des M. levator ani) oder seitliche Beckenwandmuskulatur infiltriert zu sein schien. Ein LARC, dass in den inneren Analsphinktermuskel ( $M$. sphincter ani internus) einbrach, wurde als cT3-Karzinom eingestuft (Beets-Tan et al. 2018). Karzinome mit dem Verdacht auf intramesorektale Lymphknotenmetastasen (LKM) wurden als CUICC-III Stadium gewertet.

\subsection{Perioperative multimodale Behandlung}

Die Indikationsstellung zur Durchführung einer perioperativen MMT erfolgte nach Vorstellung des Patienten im multiprofessionellen Tumorboard des G-CCC. Dort wurde die interdisziplinäre Diskussion mit dem Ziel geführt, ein optimales Therapiekonzept unter Berücksichtigung einer Teilnahme des Patienten an klinischen Studien zu finden. Die Behandlungsmaßnahmen (Abbildung 2) bestanden für die 
Patientenkohorte A $(n=64)$ aus einer präoperativen CRT (RT, Gesamtdosis: 50,4 Gray (Gy), appliziert in 28 Fraktionen, Einzeldosis pro Tag 1,8 Gy, Tag (d) 15 pro Woche) mit 5-FU. Die 5-FU Gaben erfolgten simultan zur RT an d 1-d 5 und an d 29-d 33 als 120 h-ige Dauerinfusion (Dosierung von 5-FU: $1000 \mathrm{mg} / \mathrm{m}^{2} / \mathrm{d}$ ) via Port-System. Nach einem therapiefreien Intervall von fünf bis sechs Wochen wurde die TME durchgeführt. Vier bis fünf Wochen nach der TME wurde die adCTx (5-FU: $500 \mathrm{mg} / \mathrm{m}^{2}$, als i.v. Bolus über 2-5 Minuten, ein Zyklus: d 1-d 5; Wiederholung an d 29) mit insgesamt vier Zyklen eingeleitet.

Bei der Patientenkohorte B $(n=63)$ erfolgte die präoperative CRT mit 5-FU + OX (Dosierung 5-FU: $250 \mathrm{mg} / \mathrm{m}^{2} / \mathrm{d}$, Dauerinfusion an den d 1-d 14 und d 22-d 35 via Port-System; Dosierung OX: $50 \mathrm{mg} / \mathrm{m}^{2}$ an d 1, d 8, d 22 und d 29 als $2 \mathrm{~h}$ i.v. Infusion in $500 \mathrm{ml}$ Glukose $5 \%$ ). Nach einem therapiefreien Intervall von fünf bis sechs Wochen, einer TME und einer erneuten Therapiepause von vier bis fünf Wochen wurde die adCTx mit 5-FU + OX durchgeführt (OX: $100 \mathrm{mg} / \mathrm{m}^{2}$ i.v. als $2 \mathrm{~h}$ Infusion in $500 \mathrm{ml}$ Glukose $5 \%$, d 1; FS: $400 \mathrm{mg} / \mathrm{m}^{2}$ als $2 \mathrm{~h}$-Infusion, d 1; 5-FU: $2400 \mathrm{mg} / \mathrm{m}^{2}$ als $46 \mathrm{~h}$-Infusion mittels Baxter®-Pumpe via Port-System (Abbildung 9); Wiederholung d 15, insgesamt acht Applikationen (vier Zyklen).

In der Patientenkohorte $C(n=50)$ erhielten die Patienten eine präoperative CRT analog der Kohorte B (5-FU: $250 \mathrm{mg} / \mathrm{m}^{2} / \mathrm{d}$, c.i.v. via Port-System an d 1-d 14 und an d 22-d 35; OX: $50 \mathrm{mg} / \mathrm{m}^{2}$, i.v. appliziert über $2 \mathrm{~h}$, an $\mathrm{d} 1$, d 8, d 22 und d 29) gefolgt von drei Gaben einer modifizierten FOLFOX-CTx (FS: $400 \mathrm{mg} / \mathrm{m}^{2}$, i.v. über $2 \mathrm{~h}$, an d 1, d 15 und d 30; 5-FU: $2400 \mathrm{mg} / \mathrm{m}^{2}$, c.i.v. über $46 \mathrm{~h}$ Infusion über Baxter®-Pumpe, an d 1, d 15 und d 30; OX: $100 \mathrm{mg} / \mathrm{m}^{2}$, i.v. über $2 \mathrm{~h}$, an $\mathrm{d} 1, \mathrm{~d} 15$ und d 30). Nach einem therapiefreien Intervall von vier bis fünf Wochen wurde eine TME durchgeführt. 
Patienten-Kohorten A bis C - Multimodale Therapie beim Rektumkarzinom

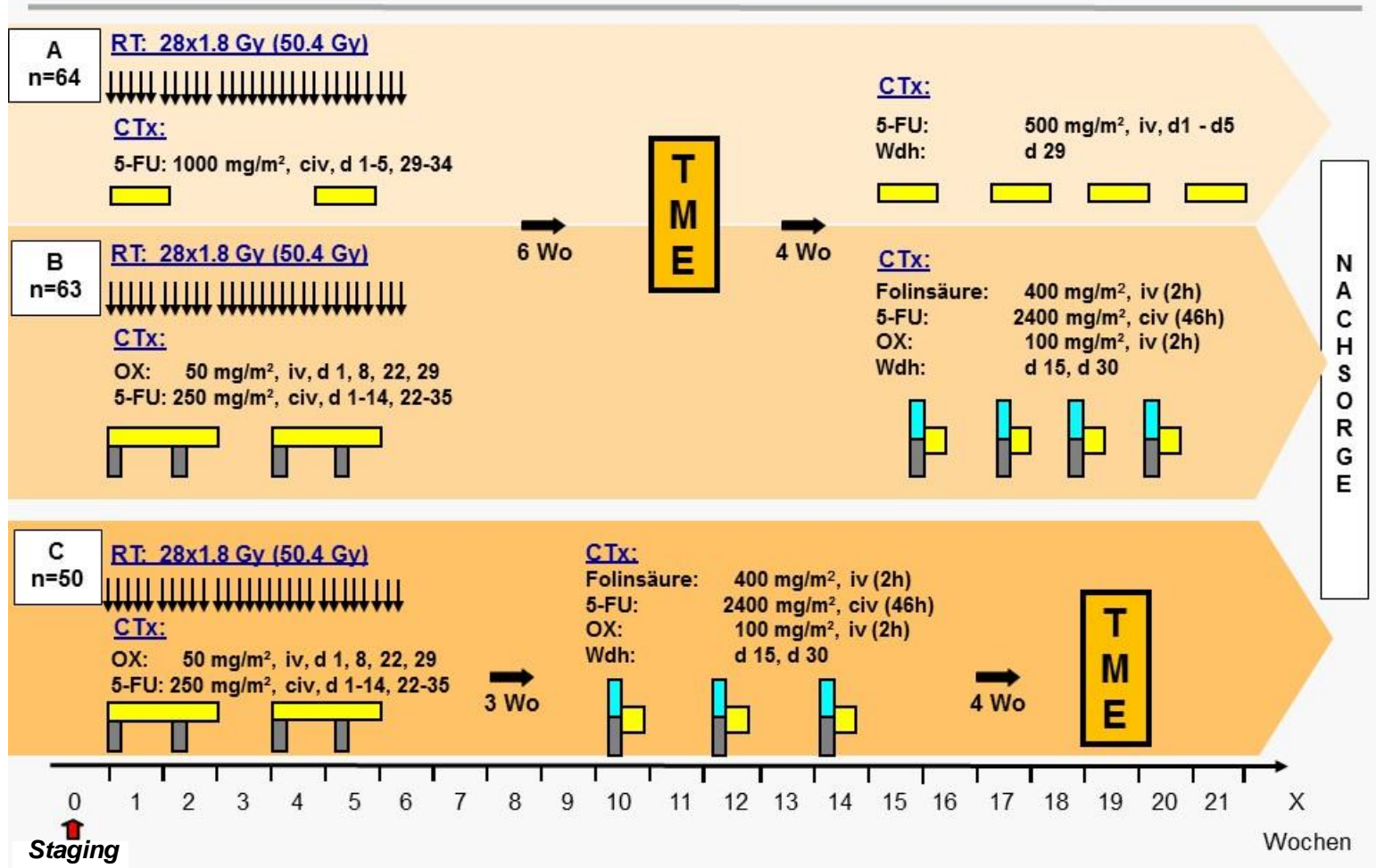

Abbildung 2: Perioperative Multimodaltherapie - Patientenkohorten A bis $\mathrm{C}$

Eigene Darstellung nach den Studienprotokollen; Abkürzungen: RT: Radiatio, alle Patienten erhielten eine Strahlentherapie mit einer Gesamtdosis von 50,4 Gy; CTx: Chemotherapie; OX: Oxaliplatin; 5-FU: 5-Fluorouracil; Folinsäure (FS): FS + 5-FU + OX (FOLFOXCTx); TME: totale mesorektale Exzision; civ: kontinuierliche intravenöse Infusion via PortSystem; iv: intravenös, WdH: Wiederholung; d: Tag der Applikation; Wo: Wochen

\subsection{Chirurgische Behandlung}

Nach Abschluss der präoperativen MMT wurde bei allen Patienten eine TME durchgeführt. Die Rektumkarzinome wurden en-bloc mit dem umgebenden mesorektalen Weichgewebe im Rahmen kontinenzerhaltender tiefer anteriorer Rektumresektionen (TARR) entfernt. Bei abdomino-perinealen Rektumresektionen (APR) wurden zusätzlich das gesamte extraperitoneale/perineale Fett- und Lymphgewebe oberhalb der Ebene des M. levator ani reseziert (Sprenger et al. 2016; Peeters et al. 2007; Heald 1998; Heald et al. 1998; Enker et al. 1997; Heald et al. 1982). In Einzelfällen war die Mitresektion des M. levator ani i. S. einer erweiterten extralevatorischen und zylindrischen APR erforderlich (West et al. 2008; Holm et al. 2007). 
Die Festlegung des jeweiligen OP-Verfahrens erfolgte nach den Staging-Befunden anhand der anatomischen Lage und des Ausmaßes des Rektumkarzinoms. Grundsätzlich hatte die Einhaltung eines ausreichenden Sicherheitsabstands zwischen dem Rektumkarzinom und der chirurgischen Resektionsebene Vorrang. Sphinktererhaltende Verfahren einschließlich der intersphinktären Resektion mit koloanaler Anastomose wurden nur bei Gewährleistung eines makroskopischen Sicherheitsabstandes von $>1,5 \mathrm{~cm}$ (nach distal) favorisiert. Die Qualität der TME wurde perioperativ (ex situ) von Chirurgen beurteilt (Anhang 6.6.1).

\subsection{Untersuchung des Rektumresektats}

Postoperativ wurde die Qualität der TME auch durch Pathologen beurteilt. Unter Anwendung der MERCURY-Kriterien wurde die Unversehrtheit der mesorektalen Hüllfaszie gegenüber der intramesorektalen und LMP-Resektionsebene bewertet (Hermanek et al. 2015; MERCURY Study Group 2007; Quirke 2003; Hermanek et al. 2003; Merkel et al. 2001; Nagtegaal et al. 2002b; Quirke et al. 1986). In Fällen einer APR wurde zusätzlich die chirurgische Resektatoberfläche im extraperitonealen/-perinealen Bereich beurteilt (Kitz et al. 2018; Rödel et al. 2015; 2012; Quirke et al. 2009; Nagtegaal et al. 2005; Anhang 6.6.2). Eine im Rektumresektat nachweisbare L-, V- oder Pn-Tumorzellinfiltration wurde als ypL-/V-/Pn-positiv klassifiziert. Zudem wurde die extramurale Tumorausdehnung (ETS, extramural tumor spread), d. h. die Ausbreitung von Karzinomzellverbänden über die LMP ins Mesorektum hinausgehend, bei allen T3-Tumoren gemessen und nach präoperativer CRT als урTЗа (<1 mm Tumorzellinfiltration), урT3b $(<5 \mathrm{~mm})$, урT3с $(<15 \mathrm{~mm})$ und ypT3d $(\geq 15 \mathrm{~mm}$ ) Status eingeteilt. Die proximalen, lateralen und distalen Resektionsränder wurden einzeln beurteilt und bei mikroskopisch karzinomfreien Schnitträndern als R0-Residualstatus gewertet. Ein R1-Status lag bei einem mikroskopisch nachweisbaren Tumorkontakt zur chirurgischen Schnittebene vor. Ein R2-Status wurde angegeben, sofern intraoperativ makroskopisch Karzinomgewebe in situ verblieben war (Tabelle 7 und A12). Der zirkumferentielle Resektionsrand (CRM) wurde als negativ eingestuft, sofern der Abstand zwischen den Karzi- 
nomzellen und dem Resektionsrand $\geq 2 \mathrm{~mm}$ betragen hatte (Nagtegaal et al. 2017; 2002a; Fokas et al. 2014; Glynne-Jones et. al. 2006). Die Therapieinduzierte Tumorregression wurde mit Hilfe eines fünfstufigen Tumor-RegressionGrading-Systems (TRG-System) beschrieben (Dworak et al. 1997) (Anhang 6.5.4). Als gutes Tumoransprechen (GR; good response) auf die MMT wurden jene Fälle gewertet, bei denen ein TRG-3 oder ein TRG-4 vorlag. Fälle mit einem TRG-1- oder TRG-2-Status wurden als partielles Ansprechen (PR, partial response) dokumentiert. Die Therapie-induzierte Fibrose wurde als prozentualer Anteil am Tumorgewebe (\% im Sichtfeld des Lichtmikroskops) bestimmt.

\subsection{Postoperative Behandlung}

Für die Patienten der Kohorten $A$ und $B$ waren vier bis sechs Wochen nach der TME die Einleitung einer adCTx mit 5-FU oder mit 5-FU + FS + OX (mFOLFOX) vorgesehen gegenüber der alleinigen Nachbeobachtung in Kohorte $C$ (Abbildung 3). Bei zehn Patienten konnte nach der TME keine Zuteilung zu den postoperativen Therapiegruppen erfolgen. Die Gründe dafür sind in Tabelle A1 aufgeführt. Zudem erhielten neun Patienten der Kohorte A (Patient H2O, H30; H35, H41, H50, $\mathrm{H} 71, \mathrm{H} 79, \mathrm{H} 88, \mathrm{H} 168$ ) eine adCTx nach dem Behandlungsregime der Kohorte B (Abbildung 3). Zwei Patienten (Patient H63, H83) aus der Kohorte B wurde 5-FU Monotherapie nach dem Protokoll der Kohorte A verabreicht. Insgesamt starteten 52 Patienten mit 5-FU und 66 Patienten mit mFOLFOX die adCTx. 


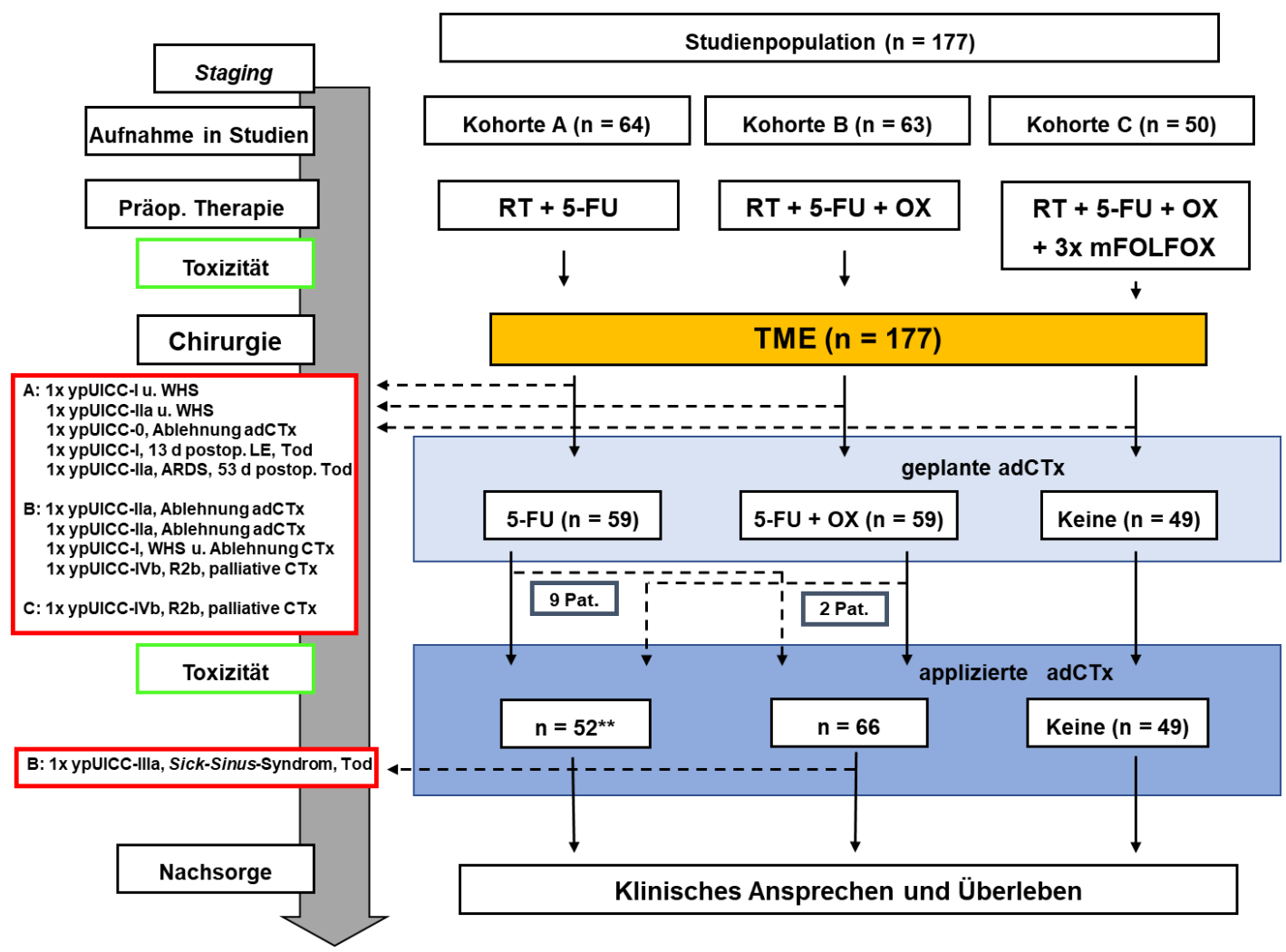

Abbildung 3: Patientenkohorten und erfolgte Therapie

Eigene Darstellung; adCTx: postoperative additive Chemotherapie; OX: Oxaliplatin; 5-FU: 5-Fluorouracil; mFOLFOX: modifizierte CTx mit Folinsäure (FS), 5-FU und OX; ${ }^{* *}$ : bei einem Patienten wurden statt infusionalem 5-FU äquivalente Dosen an oralem 5-FU (Xeloda $\left.{ }^{\circledR}\right)$ verabreicht; rot umrandet: Patienten mit schwerer Protokollverletzung (Tabelle A1, Anhang 6.1) oder Common Toxicity Criteria(CTC)-Grad 5 (Tabelle 22); blau umrandet: Therapiewechsler zwischen den Kohorten $\mathrm{A}$ und $\mathrm{B}$

\subsection{Chemotherapie - eingesetzte Substanzen}

In den Patientenkohorten A, B und C wurden verschiedene CTx-Regime durchgeführt. Die jeweilige Dosis wurde Körperoberflächen (KOF) adaptiert ermittelt nach der Formel: $A=0,007184 \mathrm{~m}^{2} \times(\mathrm{h} / \mathrm{cm})^{0,725} \times(\mathrm{m} / \mathrm{kg})^{0,425}(\mathrm{Du}$ Bois und $\mathrm{Du}$ Bois 1989). Dabei wurde die KOF (A: xy $\mathrm{mg} / \mathrm{m}^{2}$ ) aus der Körpergröße ( $\mathrm{h}$ in $\mathrm{cm}$ ) und dem Körpergewicht ( $\mathrm{m}$ in $\mathrm{kg}$ ) bestimmt. In Fällen mit einer KOF $>2 \mathrm{~m}^{2}$ wurde eine maximale KOF von 2,1 $\mathrm{m}^{2}$ zur CTx-Dosisberechnung festgelegt (sogenanntes „dose-capping“). Im Folgenden werden die CTx-Arzneistoffe in ihrer Struktur, Wirkung, Applikationsform und ihren Nebenwirkungen veranschaulicht (Abbildung 4). 


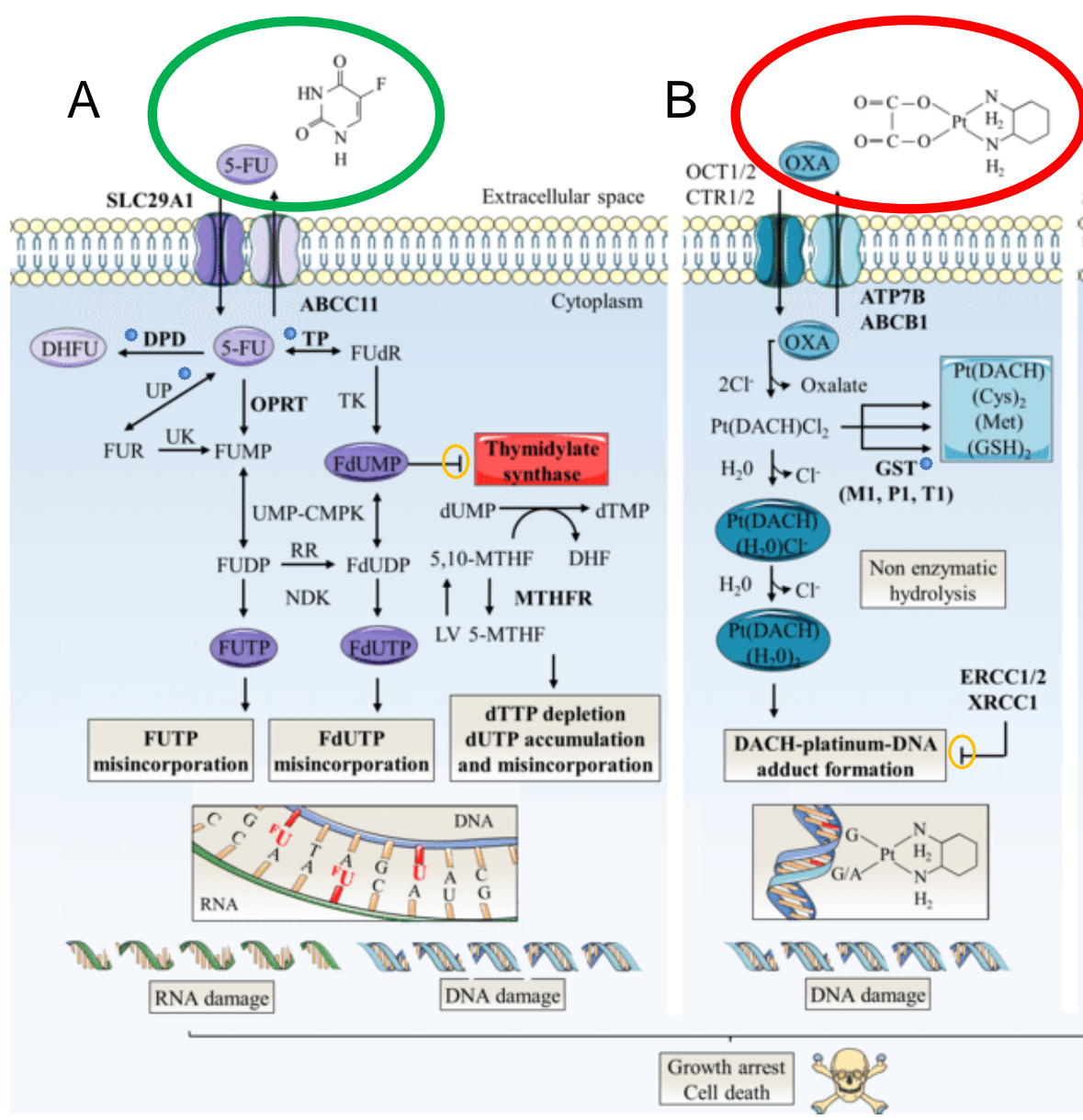

Abbildung 4: Wirkmechanismen von 5-FU und Oxaliplatin (OX) bei KRK

Modifizierte Abbildung nach Very et al. (2018); schwarze Pfeile: Metabolisierungsweg; Strichlinien mit gelbem Kreis: Hemmung des chemischen Prozesses (OX-Weg) oder der Enzymfunktion; blaue Punkte: anaboler Metabolisierungsweg; fett gedruckt: Biomarker für CTx-Resistenz. 5-MTHF: 5-Methyltetrahydrofolat; Cl: Chlor; DHF: Dihydrofolat; dTTP: Desoxythymidintriphosphat; dUTP: Desoxyuridintriphosphat; FdUDP: Fluordesoxyuridindiphosphat; 5-FU:5-Fluorouracil; FUDP: Fluoruridindiphosphat; FUMP: Fluoruridinmonophosphat; FUR: Fluoruridin; MTHFR: Methylentetrahydrofolatreduktase; NDK: Nucleosiddiphosphatkinase; Pt $(\mathrm{DACH})\left(\mathrm{Cl}_{2}\right)$ : Dichlor-DACH-Platin; Pt $(\mathrm{DACH})\left(\mathrm{H}_{2} \mathrm{O}\right)$ 2: DiaquoDACH-Platin; Pt (DACH) $\left(\mathrm{H}_{2} \mathrm{O}\right)$ Cl: Monoaquo-DACH-Platin; RR: Ribonukleotidreduktase; UK: Uridinkinase; UMP-CMPK: Uridinmonophosphat/Cytidinmonophosphat-Kinase; UP: Uridinphosphorylase.

A: grüner Kreis mit 5-FU-Strukturformel; 5-FU-Metabolismus: 5-FU dringt über SLCTransporter (Nucleoside Solute Carrier), wie SLC29A1, in die Tumorzelle. Es wird in die Hauptmetabolite Fluordesoxyuridinmonophosphat (FdUMP), Fluordesoxyuridintriphosphat (FdUTP) und Fluoruridintriphosphat (FUTP) umgewandelt. FdUMP verringert die Biosynthese von Pyrimidinnukleotiden durch Hemmung von Thymidilatsynthase (TS). Die TS katalysiert als Enzym die reduktive Methylierung von Desoxyuridinmonophosphat (dUMP) zu Desoxythymidinmonophosphat (dTMP) unter Verwendung von 5,10Methylentetrahydrofolat (5,10-MTHF). FdUMP hemmt die DNA-Synthese und -Reparatur mit der Folge eines DNA-Strangbruchs und einer Apoptose-Induktion. 5-FU kann auch in die DNA (über die FdUTP) oder RNA (über das FUTP) eingebaut werden mit einer nach- 
folgenden DNA-Fragmentierung und Reduktion der Proteinbiosynthese. Die Folinsäure (FS) erhöht den intrazellulären Gehalt an 5,10-MTHF und steigert so auch die Toxizität von 5-FU. DPD (Dihydropyrimidindehydrogenase) wandelt 5-FU zu inaktivem Dihydrofluoruracil (DHFU) um. 5-FU-Metabolite werden durch ABC-Transporter, wie ABCC11, aus der Zelle geschleust. Intrazelluläre TS- und OPRT-Überexpressionen sind wichtige molekulare Marker für eine 5-FU-Resistenz.

B: roter Kreis OX-Strukturformel); Oxaliplatin-Metabolismus: OX, wird von organischen Kationen (OCT1/2) und Kupfer (CTR1/2) in die Zelle transportiert. ATPase (ATP7A/B)Transporter vom P-Typ und ABC-Transporter fördern ihren Ausstrom. Durch Hydrolyse erfolgt die Umwandlung von OX in die aktiven Metaboliten: Monoaquo-1,2-diaminocyclohexan (DACH)-Platin, Diaquo-DACH-Platin mit der Folge einer Alkylierung der DNA (G-/ G- oder G-/A-Intra-Strang-Vernetzungen), einer Blockade der DNA-Replikation-/Transkription und der Einleitung der Apoptose. (CC BY 3.0)

\subsubsection{5-Fluorouracil (5-FU)}

5-Fluorouracil (5-FU, Firma: Medac, Gesellschaft für klinische Spezialpräparate $\mathrm{GmbH}$, Hamburg, Deutschland; Arzneimittelfachinformation im Anhang 6.7) hat die Strukturformel $\mathrm{C}_{4} \mathrm{H}_{3} \mathrm{FN}_{2} \mathrm{O}_{2}$ (Longley et al. 2003) und die molare Masse von 130,08 g/mol. 5-FU ist ein Pyrimidinantagonist und gehört zur Gruppe der Antimetabolite (Heidelberger et al. 1957).<smiles>O=c1[nH]cc(F)c(=O)[nH]1</smiles>

Abbildung 5: 5-FU-Strukturformel

Bereits vor über 40 Jahren wurde die Synthese von 5-FU aus der Biotransformation von Fluoracetat (Salz der Fluoressigsäure) zu Fluorcitrat als heterocyclische organische Verbindung beschrieben (Heidelberger 1975; Elliort und Birch 1972). Diese Verbindung, bestehend aus einem Pyrimidingrundgerüst mit zwei Carbonylgruppen und Fluor, bildet ein Derivat von großer Strukturähnlichkeit mit der Nukleinbase Uracil. Wird dieses Derivat als funktionsloser Antimetabolit während der DNA-/RNA-Replikation anstelle der Pyrimidinbasen Cytosin und Thymidin (DNA-Nukleotide) bzw. Uracil (RNA-Nukleotid) eingebaut, so kommt es zu einer Hemmung der Zellteilung (Abbildung 4). Letztlich ist 5-FU ein Prodrug, d. h. 5-FU wird erst intrazellulär in aktive Nukleotide, wie z. B. das FluorouridinMonophosphat(5-FUMP), umgewandelt. Dieses Nukleotid wird entweder über eine 
Kinase in Fluorouridin-Monophosphat (5-FUTP) oder über eine Reduktase in 5-Fluorodesoxyuridin-Monophosphat (5-FdUMP) und weiter in FluorodesoxyuridinTriphosphat (5-FdUTP) metabolisiert. 5-FU wird als "falscher” Metabolit (5-FdUMP) in die DNA-/RNA-Replikation eingebaut (Pizzorno et al. 1997) und führt zur Hemmung der zelleigenen Thymidilatsynthase (TS). Diese bewirkt die Umwandlung von Uridin in Thymin (Walther et al. 2009; Locker et al. 2006). Zudem ist die TS essentiell für die DNA-Synthese und das Zellwachstum. Somit wirkt 5-FdUMP in der Interphase des Zellzyklus und hemmt neben der DNA-/RNASynthese und -Reparatur auch den für die Zellen lebensnotwendigen ExosomKomplex (Fang et al. 2004). Es kommt zu Einzelstrangbrüchen in der DNA, einem Stopp der Proteinbiosynthese (Liao et al. 2005; Lönn und Lönn 1984; Mandel 1981) und zum Zelltod durch Apoptose-Induktion (Abbildung 4).

Die enzymatische Elimination von 5-FU findet vorwiegend in der Leber statt und nur $20 \%$ des intravenös applizierten 5-FU werden unverändert renal ausgeschieden (Mutschler et al. 2008; Mutschler 2001; Pinedo und Peters 1988). Die Metabolisierung von 5-FU (normale Halbwertszeit: 10-15 min) kann aber durch Genpolymorphismen in den abbauenden Enzymen (z. B. der Dihydropyrimidindehydrogenase und der TS) erheblich verlängert sein (pathologische Halbwertszeit: $159 \mathrm{~min}$ ) (Saif et al. 2009).

Die kumulative Anflutung von intrazellulärem 5-FU führt zu einer stärkeren zytostatischen Wirkung sowie erhöhten Nebenwirkungen. Mit dem 5-FU induzierten Absterben von Epithelzellen kann es zu starken Entzündungsreaktionen an Schleimhäuten und zur Knochenmarkdepression kommen. Zudem zeigen 1,6\% aller Patienten unter 5-FU-Therapie eine Angina Pectoris-Symptomatik oder entwickeln eine Myokardischämie. Bei Patienten mit vorbekannten Herzerkrankungen beträgt der Anteil an schweren Koronargefäßspasmen und Vaskulitiden sogar 4,5\% (Sara et al. 2018; Scheffold et al. 2005; Labianca et al.1982). 


\subsubsection{DPD-Exon 14 Skipping Mutationstest}

Eine zentrale Rolle im 5-FU-Stoffwechsel spielt die auf Chromosom 1 p22 lokalisierte Dihydropyrimidindehydrogenase (DPD) (Abbildung 4 und A1). Dieses Enzym verstoffwechselt $>80 \%$ des applizierten 5 -FU, wobei die DPD-Aktivität intraund interindividuell stark schwanken kann (Adjei et. al 2002). Eine Exon-14Skipping-Punktmutation der DPD verursacht ein inaktives Enzym. In der Folge kann in therapeutischer Dosis verabreichtes 5-FU von DPD-Mutationsträgern nur schlecht abgebaut werden. Die kumulative Anflutung von 5-FU wirkt stark toxisch und kann zu schweren bis lebensbedrohlichen Nebenwirkungen führen, wie z. B. zur febrilen Neutropenie, zu progredient ulzerierenden Mukositiden, zur kardialen Dekompensation infolge rezidivierender Koronarspasmen und zu schweren neurologischen sowie gastrointestinalen Begleiterscheinungen (Capitain et al. 2008; Locker et al. 2006; Wei et al. 1996). Etwa 40 \% der heterozygoten Merkmalträger einer Exon-14-Skipping-Mutation erleiden Toxizitäten mit Common Toxicity Criteria(CTC)-Grad 4 (van Kuilenburg 2004).

In Europa ist bei $5 \%$ der Bevölkerung ein partielles und in 0,2\% ein komplettes DPD-Defizit bekannt (Saif et al. 2009; Walther et al. 2009). Es wurden bisher $>30$ autosomal vererbbare DPD-Genpolymorphismen identifiziert, die für eine reduzierte DPD-Aktivität verantwortlich gemacht werden können (Saif et al. 2009). Durch den Einsatz eines DPD-Exon-14-Skipping-Mutationstests können $>35 \%$ aller 5-FU-induzierbaren, schweren Toxizitätsfälle vorhergesagt werden. Wird der Nachweis einer heterozygoten Mutationsträgerschaft geführt, so kann dem Patienten nach Überprüfung der Indikationsstellung zur 5-FU-haltigen CTx nur eine strengstens überwachte 5-FU-Dosisermittlung empfohlen werden. Nach der 5-FU Einzeltestdosis kann der Patient durch Messungen des Dihydrouracil/UracilVerhältnisses und der 5-FU Plasmaclearance als tatsächlicher Risikopatient identifiziert werden (Gamelin et al. 2007). Bei $17 \%$ der Patienten mit phänotypisch reduzierter DPD-Aktivität kann eine molekulargenetische Ursache nachgewiesen werden (Gamelin et al. 2007). Eine Kontraindikation zur CTx mit 5-FU CTx besteht immer bei komplett homozygotem DPD-Mutationsstatus, der in < 0,1 \% der Bevölkerung vorliegt (Boisdron-Celle et al. 2007). 
Bei allen Patienten der vorliegenden Dissertation waren prätherapeutisch DPDExon-14-Skipping-Mutationsteste zum Ausschluss eines homozygoten DPD-Mutationsstatus erfolgt. Zur Durchführung eines Tests waren den Patienten 4 ml EDTAVollblut abgenommen worden (Raida et al. 2001; van Kuilenburg et al. 2000; 1999).

\subsubsection{Applikationsformen und Nebenwirkungen von 5-FU}

5-FU kann als kontinuierliche Gabe (über 46-48 h) über ein subkutan implantiertes, zentralvenöses Port-Kathetersystem in einer Dosierung von $2400 \mathrm{mg} / \mathrm{m}^{2} \mathrm{KOF}$ verabreicht werden. Vor der 5-FU-Gabe erhalten die Patienten üblicherweise eine FS-Infusion zur Hemmung der intrazellulären TS und der intrazellulären Thymidinsynthese. Aufgrund des nachfolgend verzögerten 5-FU Metabolismus bewirkt die FS-Applikation aus therapeutischer Sicht deutlich höhere 5-FU Dosen ohne relevante Steigerung der Nebenwirkungen. Mit einer ambulant durchführbaren 5-FUDauerinfusion nach vorausgegangener FS-Gabe können bei Patienten mit mKRK Remissionsraten > 30 \% erreicht werden (Gramont et al. 1997; Lokich et al. 1989). Die Pharmakokinetik von 5-FU wird durch das Alter, das Geschlecht, die Organfunktionen, zahlreiche Medikamenten-Interaktionen und vom Genotypus der Patienten beeinflusst (Kaldate et al. 2012; Extermann et al. 2004; Milano et al. 1992). Die toxischen Auswirkungen von 5-FU zeigen sich als Mukositis, Stomatitis, Übelkeit, Erbrechen, Diarrhoen und Alopezie. Zudem kann 5-FU eine passagere Knochenmarkdepression mit Suppression der Erythro-, Granulo- und Thrombopoese auslösen. Besonders gefürchtet sind aber die 5-FU-getriggerten Koronargefäßspasmen, die von einer initialen Angina Pectoris-Symptomatik bis zum fulminanten Myokardinfarkt führen können (Scheffold et al. 2005). Diese kardiale Problematik kann auch unter oraler 5-FU-Medikation mit Capecitabin (Xeloda®) auftreten (Frickhofen et al. 2002). Im Gegensatz zu den im EKG ablesbaren regionalen linksventrikulären Kontraktilitätsstörungen sind komplexe Herzrhythmusstörungen unter 5-FU viel seltener zu beobachten (Patel et al. 1987). Die kardialen Symptome treten in den ersten Stunden unter 5-FU Gabe auf. Für Patienten mit einer 
vorbekannten koronaren Herzerkrankung ist das Risiko für klinisch manifeste bis lebensbedrohliche Nebenwirkungen um das 7-fache unter 5-FU Exposition erhöht (Meyer et al. 1997).

Unter der fortgesetzten Applikation hoher Dosen an 5-FU oder Capecitabin ist mit Epitheliolysen an den Akren zu rechnen. Dieses sogenannte Hand-Fuß-Syndrom zeigt sich durch erythematöse Hautveränderungen in den Handinnenflächen und an den Fußsohlen (Salzberg und Herrmann 2000). Wesentlich seltener treten unter 5-FU Schädigungen der Gliazellen auf (Han et al. 2008), die sich klinisch als zerebelläres Syndrom mit Ataxie, Dysarthrie und Dysmetrie äußern. Verantwortlich für die kardio- und neurotoxischen Schädigungen unter 5-FU-haltiger CTx (Upshaw et al. 2019; Sara et al. 2018; Polk et al. 2014; 2013; Scheffold et al. 2005; Yamashita et al. 2004; Arellano et al. 1998; Koenig und Patel 1970a; 1970b) ist der Metabolit Fluoracetat (Heidelberger 1975). Die klinische Konsequenz beim Auftreten einer 5-FU-Toxizität ist die sofortige Dosisreduktion bzw. der vorzeitige Abbruch der CTx. Zur Vermeidung höhergradiger Toxizitäten, deren Auswirkungen die Therapie-Compliance der Patienten negativ beeinflussen könnten, ist ein 5-FU-Drug-Monitoring geeignet. Untersuchungen aus der Arbeitsgruppe TP5 der KFO-179-2 zeigten, dass 5-FU Spiegelmessungen im Blutserum (5-FU-AUCSpiegelmessungen) mit Hilfe des Nanopartikelimmunoassays 5-Fluorouracil (My5-FU ${ }^{\mathrm{TM}}$ ) Assay (CE-zertifiziertes Verfahren der Firma Saladax Biomedical, Bethlehem, PA, USA) reproduzierbar gewesen sind (Quack 2015; Quack et al. 2013). Die Bestimmung der individuellen 5-FU-Metabolisierung war unabhängig vom Geschlecht der Patienten, vom Alter und einem DPD-Defizit. Interessanterweise traten höhergradige Toxizitäten beim Erreichen von 5-FU-AUC-Spiegeln im therapeutischen Zielbereich auf $(p=0,0118)$. Bei Frauen konnten deutlich höhere AUC-Spiegel erzielt $(p=0,00235)$ werden, allerdings ging ein besseres Tumoransprechen auch mit einer höheren, passageren Hämatotoxizität einher (Quack 2015). 


\subsubsection{Folinsäure}

Folinsäure (FS, folinic acid oder auch FA, folinic acetate; Firma: Pfizer Pharma PEE GmbH, Berlin, Deutschland; aktuelle Arzneimittelfachinformation, Anhang 6.7) wird auch als Citrovorum-Faktor (CF) oder Leukovorin (LV), kurz CHO-FH4, bezeichnet.<smiles>Nc1nc(=O)c2c([nH]1)NCC(CNc1ccc(C(=O)N[C@@H](CCC(=O)O)C(=O)O)cc1)N2C=O</smiles>

Abbildung 6: Strukturformel der Folinsäure

Sie hat die Strukturformel $\mathrm{C}_{20} \mathrm{H}_{23} \mathrm{~N}_{7} \mathrm{O}_{7}$ und eine molare Masse von 473,44 g/mol. FS ist das 5-Formylderivat der Tetrahydrofolsäure und stellt die aktive Form der Folsäure dar. Sie ist an zahlreichen intrazellulären Stoffwechselprozessen (z. B. Nukleotidsynthese, Aminosäurestoffwechsel) beteiligt. In der Tumortherapie KRK wird FS häufig mit 5-FU eingesetzt, um synergistisch die zytostatische Wirkung von 5-FU auf Tumorzellen zu erhöhen. Dabei erfolgen Dosierungen der FS mit 20$500 \mathrm{mg} / \mathrm{m}^{2}$ KOF (Abbildung 2) in Abhängigkeit vom jeweiligen CTx-Regime. Durch Bindung von FS an die 5-FU-metabolisierende TS wird die intrazelluläre Thymidilat-Konzentration reduziert und nachfolgend die zytostatische Wirkung von 5-FU verstärkt. Zudem kann die sequentielle i.v. Applikation von FS zur 5-FU-haltigen CTx zytotoxische Nebenwirkungen von Arzneistoffen aus der Gruppe der Folsäure-Antagonisten reduzieren. Weiterhin können mit einer niedrig dosierten FS-Gabe Folsäure-Mangelzustände ausgeglichen werden. Derzeit existieren vier Isomere der Folinsäure, die zwei Stereozentren haben: das Calciumfolinat (Calcium-Salz), das Calciumfolinat-Pentahydrat, das Natriumfolinat (Dinatriumsalz) sowie die Levofolinsäure als rein biologisch aktives L-Isomer.

Diese vier Isomere werden als Fertigarzneimittel eingesetzt (PubChem https://pubchem.ncbi.nlm.nih.gov/compound/149436; abgerufen am 12.01.2020). 


\subsubsection{Oxaliplatin}

Oxaliplatin (OX; Eloxatin®, Firma: Sanofi-Aventis Deutschland GmbH - Industriepark Höchst in Frankfurt am Main, aktuelle Arzneimittelfachinformation, Anhang 6.7) hat die Strukturformel C8-H14-N2-O4-Pt (Aktories et al. 2010) und eine molare Masse von 397,28 g/mol.<smiles>O=C1O[P]2(N[C@H]3CCCC[C@H]3N2)OC1=O</smiles>

Abbildung 7: Strukturformel Oxaliplatin

Oxaliplatin ist ein Platinanalogon der dritten Generation (Kidani et al. 1978a; 1978b). Gegenüber dem stark nephro- und ototoxischen Cisplatin wurden bei OX die Chloridionen durch Sauerstoffionen und Amino-Liganden $\left(\mathrm{NH}_{2}\right.$ statt $\left.\mathrm{NH}_{3}\right)$ ersetzt. Dies führte in Tumorgeweben zu einer veränderten parenchymatösen und intrazellulären Verteilung. Durch den neutralen 1,2-Diaminocyclohexyl-Liganden (DACH-Ligand) ist für OX eine Kreuzresistenz gegenüber Cisplatin ausgeblieben. Zudem ist ein Platin-lon als aktiver Metabolit mit dem DACH-Liganden und dem Oxalation gebunden. Dieser Komplex ist das Enantiomer cis-[oxalato (trans-L-1,2Diaminocyclohexan]platin (Bruck et al. 1984).

\subsubsection{Wirkmechanismen Oxaliplatin}

Die zytotoxische Wirkung von OX beruht auf der Biotransformation von PlatinDNA-Addukten. Diese Aquakomplexe reagieren aufgrund einer hohen Nukleophilie des Imidazolrings mit den Nukleotiden der DNA (Di Francesco et al. 2002; Graham et al. 2000; Luo et al. 1999; Chaney et al. 1995). Die Bindung der Basen an die DNA führt zur Bildung von Di-Platin-DNA-Addukten. In der Folge werden die $\mathrm{H}$-Brückenbindungen zwischen den komplementären Basen abgeschwächt und die thermische Stabilität der DNA reduziert. Aufgrund ihrer bifunktionellen Struktur können die Mono- und Diaquakomplexe zwei Nukleotide auf einem DNA-Strang (Intrastrangverbindung), zwei DNA-Strängen (Interstrangverbindungen) miteinan- 
der und die DNA mit Proteinen (Interstrang-Crosslinks) vernetzen (Mani et al. 2002, Raymond et al. 2002). Diese DACH-Liganden (1,2-DiaminoçyclohexylLigand) hindern aufgrund ihrer Konformationsänderungen Zellen daran, PlatinAddukte zu tolerieren. In der Folge werden die DNA-Transkription-/Replikation und die Proteinbiosynthese gestört. DNA-Polymerasen können sich nicht mehr ausreichend an die DNA anlagern und es kommt zu DNA-Strangbrüchen. Diese führen zur Unterbrechung des Zellzyklus und zur schnelleren, verstärkten Einleitung der Apoptose gegenüber Cis-Diamino-Platin-Addukten (Raymond et al. 1998; Saris et al. 1996; NGTATG 1992) (Abbildungen 4, 8, 10 und 11).

Außerdem zeigt OX im Vergleich zu Cis-/Carboplatin keine Interaktion mit dem zellulären DNA-Mismatch-Repair-System (MMR-System). Gerade bei KRKPatienten können Störungen im MMR-System zu Resistenzentwicklungen gegenüber Cis-/Carboplatinhaltigen CTx-Regimen führen. Demgegenüber bleibt $\mathrm{OX}$ auch bei Störungen des MMR-Systems tumortoxisch wirksam (Di Francesco et al. 2002; Scheeff et al.1999).
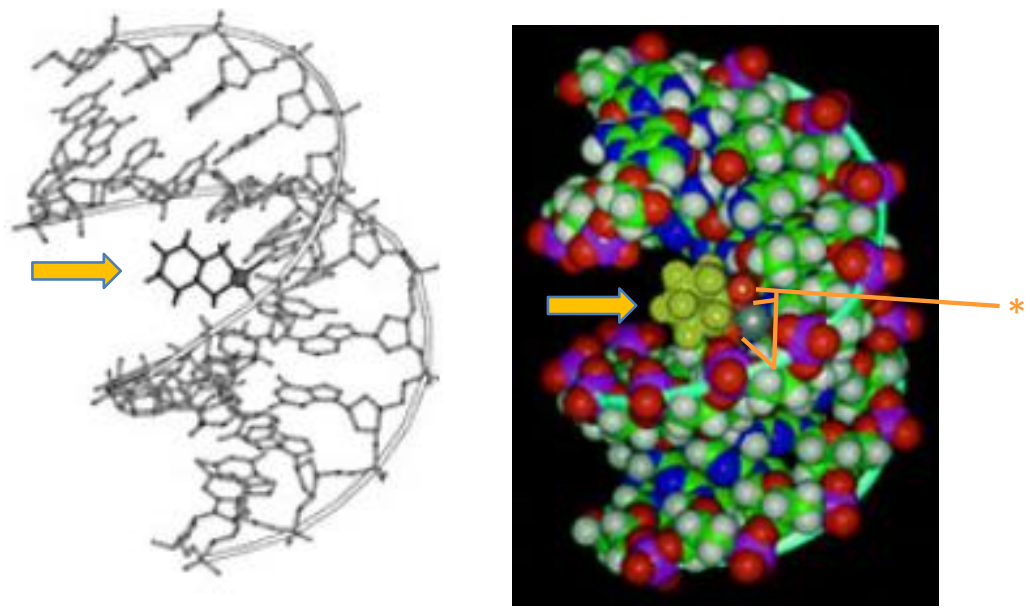

Abbildung 8: Oxaliplatin-Addukte und OX-bedingte DNA-Konformationsänderung Grün: Kohlenstoff; blau: Stickstoff; rot: Sauerstoff; weiß: Wasserstoff; magenta: Phosphor; grau: Platin; orange $\left({ }^{*}\right)$ : Amine bzw. Amin-Stickstoffe; gelb-grün: Cyclohexanring an Oxaliplatin (OX); bei der Konformationsänderung entwindet sich die DNA um ca. $80 \%$ und knickt um 20-40 \% in Richtung der "großen Furche“ ab; stärkere DNA-Furche durch den Diaminocyclohexanring (siehe Pfeile). Modifizierte Abbildung nach Scheef et al. 1999, die Verwendung erfolgt mit freundlicher Genehmigung von ASPET.

In Deutschland ist OX seit 1999 in der Therapie des mKRK zugelassen. Seit 2004 ist es auch für die postoperative adjuvante Behandlung (pUICC-III Stadien) auf- 
grund der Resultate der MOSAIC-Studie (André et al. 2004) zugelassen. In dieser Studie konnte durch die Hinzunahme von OX zur üblichen Standardbehandlung mit 5-FU das 3-Jahre DFS und das 6-Jahre OS verbessert werden. In weiteren klinischen Studien wurde die synergistische Wirkung von OX, wie z. B. im FOLFOX-4 Regime (OX: $85 \mathrm{mg} / \mathrm{m}^{2}$ KOF d 1 i.v.; 5-FU c.i.v. über $46-48 \mathrm{~h}, \mathrm{WH}$ d 14, 12 Gaben), mit 5-FU und FS bestätigt (Schmoll et al. 2015; Graeven et al. 2004). Seit 2005 ist OX bei Erwachsenen sowohl in der Erstlinientherapie von KRK im Stadium pUICC-III als auch in der Zweitlinientherapie von mKRK zugelassen.

Eine Kombination der CTx mit moAk gegen zelluläre EGF- und VEGFRezeptorstrukturen, wie z. B. mit Panitumumab (Vectibix®), Cetuximab (Erbitux®), Bevacizumab (Avastin $\AA$ ) oder Aflibercept (Zaltrap $\AA$ ), kann die Überlebensrate von Patienten mit mKRK deutlich verbessern (Hofheinz et al. 2018a). So erhöhten sich die Remissionsraten bei KRAS-Wildtyp-Patienten mit einem linksseitigen Kolonkarzinom unter FOLFOX, FOLFIRI oder 5-FU + FS in Kombination mit Cetuximab auf $55-65 \%$ gegenüber $8 \%$ unter alleiniger Cetuximab-Gabe. Ähnliches zeigte sich für Panitumumab, das als Monotherapie nur eine $10 \%$-ige Remissionsrate nach Versagen einer CTx mit FOLFOX +/- Bevacizumab erbrachte. In Kombination mit dem FOLFIRI-Regime konnten allerdings Remissionsraten von $35 \%$ erreicht werden. In der Zweitlinientherapie steigerten auch die Kombinationen von Bevacizumab mit FOLFOX- oder FOLFIRI-Regimen die Remissionsraten um $50 \%$, das PFS von 4,7 auf 6,9 Monate (HR: 0,758; $p<0,001$ ) und das OS um 1,4 Monate (Median; HR: 0,817; $p=0,0032$ ) (Stellungnahme der DGHO 2013). In der ACROBAT Phase-II Studie ergab die Kombination von FOLFOX-4 mit Cetuximab ein PFS von 12,3 Monaten (im Median, Díaz-Rubio et al. 2005) und in der CRYSTAL-Phase-I/II-Studie (Folprecht et al. 2006) ein OS von $\leq 33$ Monaten. Somit war das bis dahin bekannte 20-monatige OS von mKRK-Patienten unter einer alleinigen FOLFOX- oder FOLFIRI-CTx durch deren Kombination mit moAk deutlich verbessert worden (Reich 2006; Köhne et al. 2005; Tournigand et al. 2004; Folprecht et al. 2004; Goldberg et al. 2004; Simmonds 2000). Zudem können derartig intensivierte CTx-Regime auch ambulant, appliziert (Abbildung 9) und eine Hospitalisierung der Patienten vermieden werden. 


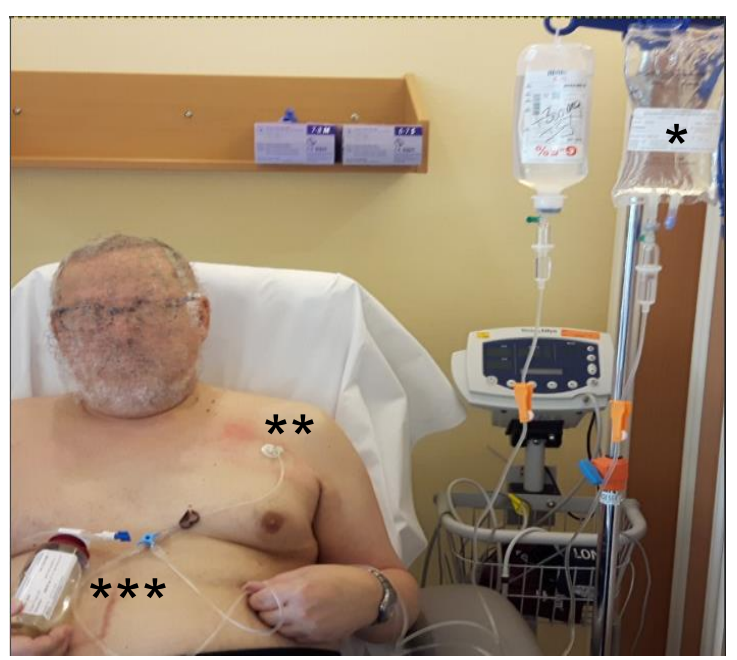

Abbildung 9: Applikation der additiven CTx via Port-System ambulant auf der IKO des G-CCC der UMG

Die additive CTx $\left(^{*}\right)$ wird den Patienten über ein links präpectoral implantiertes, zentralvenöses Portsystem $\left(^{* *}\right)$ und eine Baxter®-Pumpe $\left({ }^{* * *}\right)$ infusional auf der IKO des G-CCC der UMG appliziert. Die Veröffentlichung des Fotos erfolgte mit schriftlicher Einverständniserklärung des Patienten vom 20.05.2019

\subsubsection{Nebenwirkungen von Oxaliplatin}

Klinisch relevante, d. h. behandlungsbedürttige CTC Grade 3/4 Nebenwirkungen sind bei $>10 \%$ der Patienten unter FOLFOX-CTx zu erwarten. Sie umfassen Diarrhoen, Übelkeit, Erbrechen, Mukositiden/Stomatitiden, myelosuppressive Blutbildveränderungen (Anämie, Thrombopenie, Neutropenien der CTC-Grade 3/4) und periphere sensorische Polyneuropathien (PNP). Die Hälfte aller mit OX behandelten Patienten entwickelt eine PNP Grad 1 (Monga und O'Connell 2006). Diese Neurotoxizität unter FOLFOX kann mit Kalzium- und Magnesium-Infusionen gemildert werden (Gamelin et al. 2004). Die Verabreichung von Gabapentin (Antiepileptikum, auch wirksam gegen Neuralgien/Neuropathien) hat bei der OXassoziierten PNP keinen Stellenwert (Wong et al. 2005). Zudem können unter OX vermehrt Phlebitiden, tiefe Beinvenenthrombosen oder Lungenembolien (LE) auftreten (1 von 10 behandelten Patienten). Bei 1 von 10.000 Patienten kommt es zu Gefäßerkrankungen der Leber. Die Häufigkeit einer allergischen Vaskulitis, einer Angina Pectoris, von Herzrhythmusstörungen (QT-Verlängerung im EKG) oder eines Myokardinfarkts (z. B. linksventrikulärer Myokardinfarkt durch Koronarspas- 
men ausgelöst; Maurer et al. 1996) ist nicht sicher bekannt (Gebrauchsinformation des Herstellers Sanofi-Aventis Deutschland GmbH 2019 (Anhang 6.7); Schlitt et al. 2014).

Ein seltenes Ereignis stellt die OX-assoziierte leukozytoklastische Vaskulitis dar, die bisher nur in zwei Fällen beschrieben worden ist (Quack et al. 2013). Diese multifaktorielle, inflammatorische Erkrankung der dermalen postkapillären Venolen ist gekennzeichnet durch eine Mitbeteiligung innerer Organe, insbesondere der Nieren, des zentralen Nervensystems und/oder der Augen verbunden mit Allgemeinsymptomen wie Müdigkeit, Arthralgie und Fieber. Es sind sehr schwere Krankheitsverläufe mit akuten Glomerulonephritiden und Nierenversagen beschrieben worden, trotz des Einsatzes einer hochdosierten Corticoid-Medikation. Treten unter OX-Therapie klinische Zeichen einer Vaskulitis auf, so ist ein sofortiger Abbruch der CTx vorzunehmen. Zumindest die OX-Gabe darf nicht weitergeführt werden (Abbildungen 10 und 11; Fallbeispiel). 
A

B
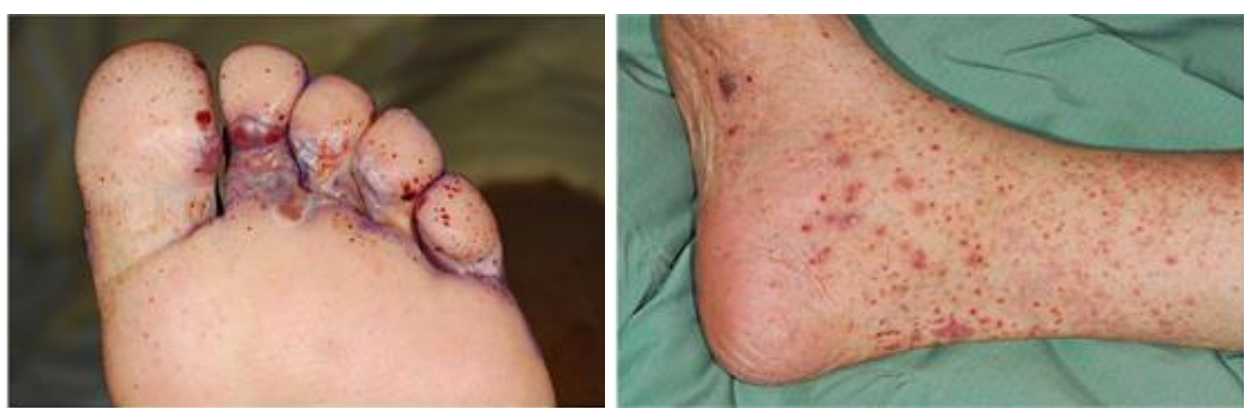

Abbildung 10: Leukozytoklastische Vaskulitis als CTx-Nebenwirkung

Leukozytoklastische Vaskulitis mit sichtbaren Hautläsionen 2 Tage nach OXApplikation (FOLFOX-CTx) bei einer Patientin mit KRK. Histologie: Entzündung/Ödem mit lymphozytären Infiltraten und Ablagerungen von Zellkerntrümmern und Fibrin, Schwellung der Endothelzellen in dermalen Venolen. Klinische Symptome: Purpura an den Zehen (Abbildung 10A) und Unterschenkeln (Abbildung 10B) (Quack et al. 2013) (CC-BY-NC 3.0)

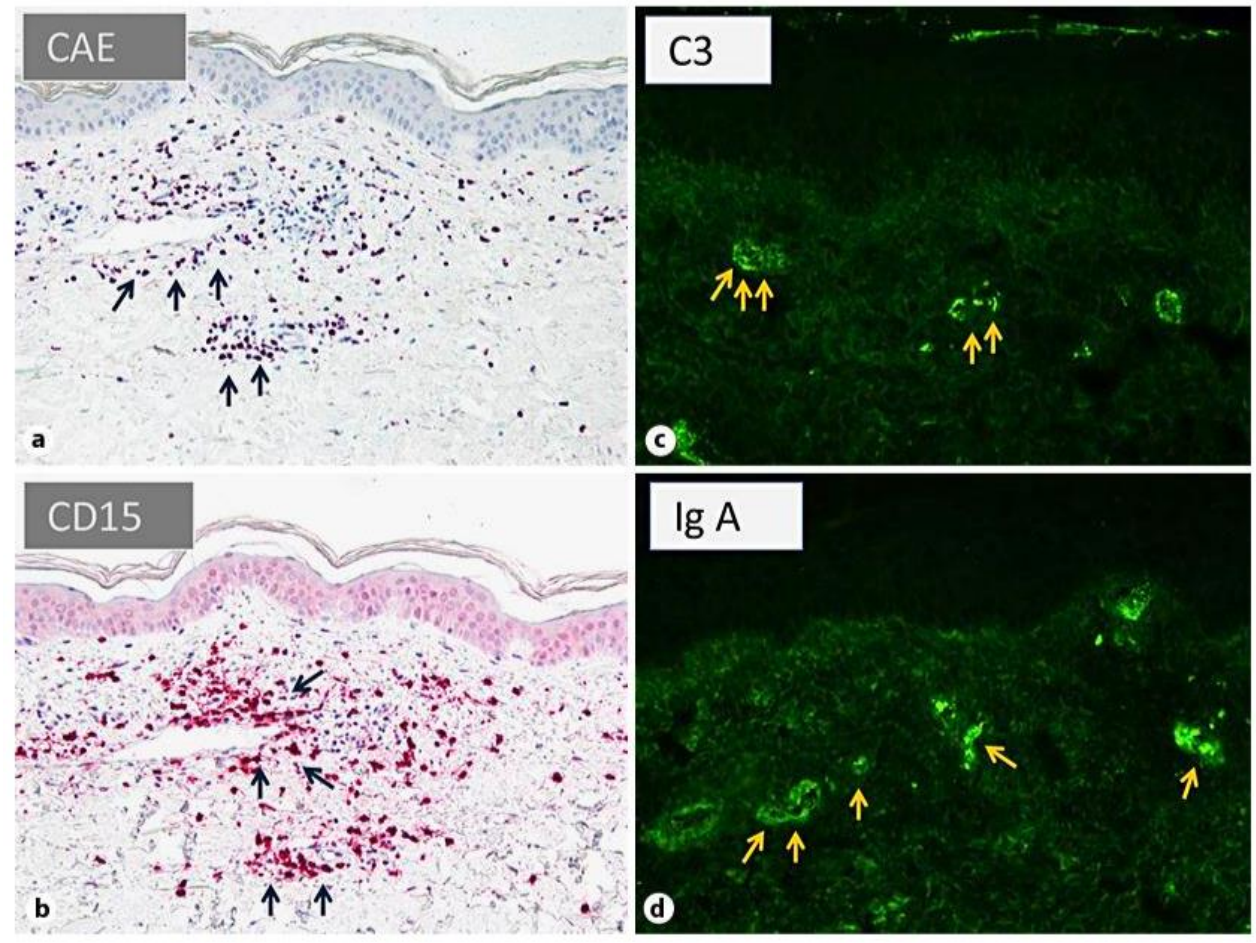

Abbildung 11: Leukozytoklastische Vaskulitis - Immunhistochemie/-fluoreszenz Immunhistochemische Färbungen gegen Chloroacetatesterase (CAE) und direkte Immunfluoreszenz-Analyse gegen den Komplementfaktor C3 und IgA an Hautbiopsien aus den Vaskulitisläsionen. Die CAE-Färbung (a) und die IHC-Färbung gegen CD15 (b) zeigen eine Anhäufung von Granulozyten um kleinste Gefäße. Die Immunfluoreszenz weist den Komplementfaktor C3 (c) und IgA-Ablagerungen (d) in kleinsten Gefäßstrukturen der papillären Dermis nach Quack et al. (2013). (CC-BY-NC 3.0) 


\subsection{RT-/CTx-Dosisdichte sowie auftretende Nebenwirkungen}

Während der perioperativen MMT aufgetretene Nebenwirkungen wurden nach der Common Terminology Criteria for Adverse Events (CTCAE) Version 4.03 (gültig seit dem 14.06.2010) des U.S. Department of Health and Human Services (National Institutes of Health, National Cancer Institute ( $\mathrm{NCl}$ 2010), bewertet und dokumentiert. Jede unter der perioperativen MMT notwendige Dosisreduktion wurde in papierbasierten CRFs erfasst und zentral supervidiert.

\subsection{Statistische Daten-Auswertung}

Die Datenanalyse wurde in Kooperation mit Dr. rer. nat. Andreas Leha, Institut für Medizinische Statistik der Universitätsmedizin Göttingen, durchgeführt.

\subsubsection{Patienten und auswertbare Datendokumentation}

Die untersuchten Patientenkohorten zeigten eine ausgewogene Altersverteilung (Abbildung 12). Allein in Kohorte $C$ fällt der geringere Anteil der > 69-Jährigen im Vergleich zu den Kohorten A und B auf.

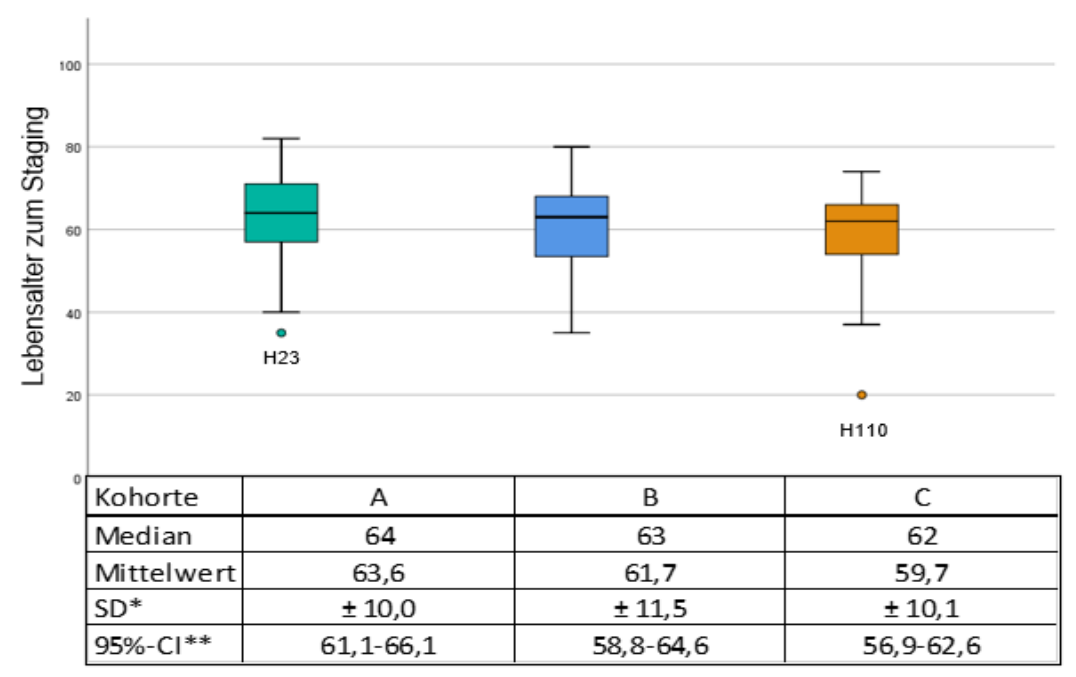

Abbildung 12: Altersverteilung der Studienpopulation Grün: Kohorte $A(n=64)$; blau: Kohorte $B(n=63)$; orange: Kohorte $C(n=50)$; durch die farbigen Punkte sind Ausreißerwerte markiert (Kohorte A: Patient H23, 35 Jahre; Kohorte

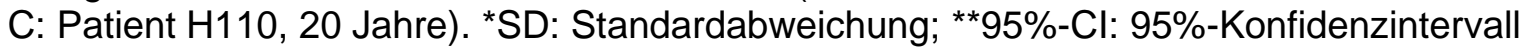




\subsubsection{Definition von Überlebensparametern}

Die Überlebensparameter wurden nach internationalen Empfehlungen definiert (Tabellen 9 und 10).

\begin{tabular}{|l|l|}
\hline Parameter & Definition \\
\hline $\begin{array}{l}\text { DFS } \\
\text { sisease-free }\end{array}$ & $\begin{array}{l}\text { Zeitraum zwischen der Rektumresektion und dem Nachweis eines LR, } \\
\text { einer FM, von sekundären Primärkarzinomen (Karzinome des Kolons oder } \\
\text { anderer Entitäten) oder des Todes (jeder Ursache); zensiert wurde ein } \\
\text { Kontaktverlust zum Patienten. }\end{array}$ \\
\hline $\begin{array}{l}\text { RFS } \\
\text { (relapse-free } \\
\text { survival) }\end{array}$ & $\begin{array}{l}\text { Zeitraum zwischen der Rektumresektion und dem Nachweis eines LR, } \\
\text { einer FM, des Todes (jeder Ursache); zensiert wurde ein Kontaktverlust. } \\
\text { lgnoriert wurden kolorektale Zweitkarzinome und neu aufgetretene } \\
\text { Karzinome anderer Entität. }\end{array}$ \\
\hline $\begin{array}{l}\text { CSS } \\
\text { (cancer-specific } \\
\text { survival) }\end{array}$ & $\begin{array}{l}\text { Zeitraum zwischen der Rektumresektion und dem Zeitpunkt des } \\
\text { Rektumkarzinom-bedingten Todes; dabei wurden beobachtete Ereignisse, } \\
\text { wie der Tod aufgrund anderer Ursachen, zensiert und ignoriert. Verstarben } \\
\text { Patienten unter einem progredienten Tumorleiden (progrediente FM) infolge } \\
\text { von Komplikationen nach interventionellen und/oder chirurgischen } \\
\text { Maßnahmen, die allein in palliativ-supportiver Intention erfolgt waren, so } \\
\text { wurde der Tod (und der Zeitpunkt des Versterbens) als Rektumkarzinom- } \\
\text { bedingt gewertet. Starben Patienten nach interventionellen und/oder } \\
\text { chirurgischen Interventionen bei stabiler Tumorerkrankung, d. h. bei FM } \\
\text { ohne eindeutigen Progress, so wurden diese Ereignisse als nicht durch das } \\
\text { Rektumkarzinom verursacht bewertet. }\end{array}$ \\
\hline $\begin{array}{l}\text { Zeitraum zwischen der Rektumresektion bis zum Auftreten eines } \\
\text { Ereignisses oder bis zum letzten Beobachtungszeitpunkt; ignoriert wurden } \\
\text { LR, FM, Zweitkarzinome im Kolon und primäre Zweitkarzinome anderer } \\
\text { Entität. Als Ereignis gewertet wurden der Tod verursacht durch das } \\
\text { Rektumkarzinom, der Tod aufgrund einer anderen Ursache, der nicht } \\
\text { krebsbedingte sowie der therapiebedingte Tod. Zensiert wurde der } \\
\text { Kontaktabbruch zum Patienten. }\end{array}$ \\
\hline $\begin{array}{l}\text { OS } \\
\text { (overall survival }\end{array}$ \\
\hline
\end{tabular}

Tabelle 9: Überlebensparameter nach Konsensusvereinbarungen

Tabelle modifiziert nach Bonnetain et al. 2017; Birgisson et al. 2011; Punt et al. 2007. 


\begin{tabular}{|l|c|c|c|c|}
\hline Ereignis: & DFS & RFS & CSS & OS \\
\hline Lokalrezidiv & E & E & I & I \\
\hline Fernmetastasen & E & E & I & I \\
\hline Zweitkarzinom, KRK & E & I & I & I \\
\hline Zweitkarzinom anderer Entität & E & I & I & I \\
\hline Tod durch Rektumkarzinom & E & E & E & E \\
\hline Tod durch Karzinom anderer Entität & E & E & C & E \\
\hline Tod, nicht Karzinom verursacht & E & E & C & E \\
\hline Therapie-assoziierter Tod & E & E & C & E \\
\hline Kontaktverlust (in der Nachsorge) & C & C & C & C \\
\hline
\end{tabular}

Tabelle 10: Überlebensparameter - Ereignisse und Endpunkte

$\mathrm{E}$ : Ereignis (event); C: zensiertes Ereignis (censored event); I: ignoriertes Ereignis (ignored event); Kontaktverlust: Kontaktabbruch zum Patienten (loss to follow-up); eine weitere Datenerhebung in dem geplanten Nachsorge-Zeitraum war nicht möglich. Somit wurde der letzte Beobachtungszeitpunkt dokumentiert und bewertet (Birgisson et al. 2011; Punt et al. 2007).

In die präsentierten Überlebenszeitanalysen gingen allein Patienten mit einem vom Pathologen bestätigten R0-/R1-Status nach TME ein. Dabei wurden immer die lokoregionäre und systemische Malignomsituation berücksichtigt. Einzelfälle, bei denen nach erfolgter Rektumresektion mit lokalem R0-Status aufgrund von synchronen Lebermetastasen in vivo ein tatsächlicher R2a-Status vorlag und bei denen später innerhalb von vier Monaten nach der Rektumresektion eine komplette Metastasenresektion durchgeführt werden konnte, wurden in den Überlebenszeitanalysen berücksichtigt und gekennzeichnet (Tabelle A6).

Insgesamt wurden 177 Patienten in die Auswertungen eingeschlossen (Tabelle 11). Alle Patienten hatten die geplante präoperative CRT +/- mFOLFOX-CTx erhalten. Nach der präoperativen MMT und einem Re-Staging war bei allen Patienten eine qualitätskontrollierte TME erfolgt. Postoperativ war eine adCTx für die Patienten der Kohorte A (5-FU) und Kohorte B ( $F S+5-F U+O X)$ gegenüber der alleinigen Beobachtung in Kohorte $C$ vorgesehen. Zehn Patienten standen für die adCTx nicht zur Verfügung (Tabelle A1). Bei den verbliebenen 167 (94,4\%) Studienteilnehmern wurde bei 11 Patienten ein Wechsel der ursprünglich geplanten adCTx durchgeführt ( $9 x$ von Kohorte $A$ nach $B ; 2 x$ von Kohorte $B$ nach $A$ ). Letztlich wurden 52 Patienten mit 5-FU adCTx und 66 Patienten mit mFOLFOX adCTx weiter behandelt. Ein Patient (0,9\%), der nach dem CTx-Protokoll der Kohorte B 
behandelt worden war, verstarb vor Beginn der Nachsorgephase (Tabelle A4, Patient H161).

\begin{tabular}{|c|c|c|c|c|}
\hline & Gesamt & $\begin{array}{c}\text { Kohorte A } \\
\text { 5-FU }\end{array}$ & $\begin{array}{l}\text { Kohorte B } \\
5-F U+O X\end{array}$ & $\begin{array}{c}\text { Kohorte C } \\
5-F U+O X+\text { mFOLFOX }\end{array}$ \\
\hline & n & n & n & n \\
\hline $\begin{array}{l}\Sigma \text { Patienten nach } \\
\text { Intention-to-treat (ITT) }\end{array}$ & 177 & 64 & 63 & 50 \\
\hline \multicolumn{5}{|l|}{ Auswertbarkeit bzgl. } \\
\hline Basisdaten & \multirow{3}{*}{177} & \multirow{3}{*}{64} & \multirow{3}{*}{63} & \multirow{3}{*}{50} \\
\hline Staging & & & & \\
\hline Präoperative CRT & & & & \\
\hline $\begin{array}{l}\text { Präoperative CTx mit 3x } \\
\text { mFOLFOX* nach CRT }\end{array}$ & 50 & 0 & 0 & 50 \\
\hline NWK, Toxizitäten & \multirow{4}{*}{177} & \multirow{4}{*}{64} & \multirow{4}{*}{63} & \multirow{4}{*}{50} \\
\hline Operationen & & & & \\
\hline Postoperative Komplikationen & & & & \\
\hline Pathologie & & & & \\
\hline Postoperative adCTx (ITT) & 118 & 59 & 59 & 0 \\
\hline $\begin{array}{l}\text { Postoperative adCTx } \\
\text { (as-treated) }\end{array}$ & \multirow[t]{2}{*}{118} & \multirow[t]{2}{*}{52} & \multirow[t]{2}{*}{66} & \multirow[t]{2}{*}{0} \\
\hline NWK, Toxizitäten & & & & \\
\hline Therapieabschluss & 167 & 52 & 66 & 49 \\
\hline Nachsorge & 166 & 52 & 65 & 49 \\
\hline
\end{tabular}

Tabelle 11: Patientenkohorten und auswertbare CRF-Dokumentation

ITT (intention-to-treat): Zuordnung der Patienten zu den Kohorten A bis C vor Therapiebeginn; CRT: Chemoradiotherapie; CTx: Chemotherapie; adCTx (intention-to-treat): geplante postoperative adCTx nach der initialen Zuteilung in Kohorte $A(n=59)$ und $B$ $(n=59)$; adCTx (as-treated): Patienten $(n=118)$, die mindestens einen Zyklus adCTx erhalten hatten und nach dem CTx-Regime der Kohorte $A(n=52)$ oder $B(n=66)$ behandelt wurden (inklusive der Therapiewechsler); ${ }^{*} \mathrm{mFOLFOX:} \mathrm{drei} \mathrm{Gaben} \mathrm{der} \mathrm{modifi-}$ zierten FOLFOX-CTx

\subsubsection{Testverfahren und multiparametrische Überlebensmodelle}

Insgesamt 167 Patienten (94,4 \%) der ursprünglichen Studienpopulation erfüllten die Einschlusskriterien für Überlebenszeitanalysen. Diese wurden nach Intentionto-treat- und As-treated-Kriterien bei jenen Patienten durchgeführt, die entweder die Rektumresektion und die adCTx begonnen hatten oder die den ersten Nachsorgetermin nach Therapieabschluss wahrnehmen konnten. Details werden in den jeweiligen Abbildungslegenden angegeben. 
Gruppenvergleiche in den klinischen und pathologischen Variablen wurden mit dem logrank-Test (Mantel-Cox) und Chi-Quadrat-Test durchgeführt. Mit allen betrachteten Variablen wurde nach manueller Auswahl ein multiparametrisches CoxRegressions-Überlebenszeitmodell für CSS entwickelt. Die Ergebnisse sind in den nachfolgenden Tabellen mit der Patienten- und der Ereignisanzahl, der Hazard Ratio (HR, bei multivariater Analyse), dem 95\%-Konfidenzintervall $(\mathrm{Cl})$ und den jeweiligen $\mathrm{p}$-Werten angegeben. Die Überlebenswahrscheinlichkeiten werden anhand von Kaplan-Meier-Überlebenskurven (K-M-Kurven) veranschaulicht.

Eine Korrelation zwischen klinisch-pathologischen Parametern (z. B. GR) und Überlebenszeiten (z. B. DFS) wurde mit Hilfe des Cox-Regression-Modells geprüft. Das mediane Überleben wurde mit einem Konfidenzintervall $(\mathrm{Cl})$ von $95 \%$ angegeben. Sank der Anteil an Überlebenden im Nachbeobachtungszeitraum nicht unter $50 \%$, so erfolgte die Angabe als NR (not reached). Zudem werden die Überlebensparameter als beschränkte erwartete mittlere Überlebenszeit (restriktiver Mittelwert (rMW); auch restricted mean of survival time (RMST)) gezeigt (A'Hern 2016; Buyse et al. 2016; Trinquart et al. 2016; Royston und Parmar 2013; Tang et al. 2007; Prentice 1989).

Die uni- und multivariaten Analysen zur Ermittlung eines prognostischen Einflusses klinischer Parameter erfolgten mit dem Cox-Proportional-Hazards-Modell (Cox 1972). Alle Ergebnisse wurden mit IBM® SPSS Statistics $\AA^{\circledR}$ Version 26 (IBM Corp. Armonk New York, US) und der Statistischen Programmierumgebung R (The R Project for Statistical Computing, Wien, Österreich; Version 3.4.2 verfügbar über: http://www.r-project.org) unter Verwendung des "survival"-Pakets berechnet. Alle Tests erfolgten auf einem Signifikanzniveau $\alpha=5 \%$. 


\section{$3 \quad$ Ergebnisse}

Die perioperative Multimodaltherapie wurde bei 55 (31,1\%) Frauen und 122 $(68,9 \%)$ Männern mit fortgeschrittenen Rektumkarzinomen der klinischen Stadien cUICC-II $(n=7 ; 4,0 \%)$, cUICC-III $(n=161 ; 90,9 \%)$ und cUICC-IV $(n=9 ; 5,1 \%)$ untersucht. Das mediane Alter betrug 63 Jahre (Mittelwert: $62 \pm$ 10,7 Jahre, Min.Max.: 20-82 Jahre) und der WHO-ECOG-Performance-Status $\leq 2$. Die Patienten hatten entweder eine präoperative CRT mit 5-FU (Patientenkohorte A, $\mathrm{n}=64$ ) oder mit $5-\mathrm{FU}+\mathrm{OX}$ (Kohorte $\mathrm{B} ; \mathrm{n}=63$ ) erhalten (Abbildungen 2 und 3 , Tabelle 12). Allein in der Patientenkohorte $C(n=50)$ folgten der präoperativen CRT mit 5-FU + OX nach einem Intervall von drei Wochen drei Applikationen einer präoperativen CTx mit mFOLFOX. Insgesamt waren 46,9\% $(n=83)$ der Karzinome im mittleren und 53,1\% ( $n=94)$ im unteren Rektumdrittel lokalisiert (Tabelle 12). Das prätherapeutische Staging ergab in $96,6 \%(n=171)$ einen klinischen $\geq$ cT3-Status. Bei $24,9 \%$ der Patienten wurde ein organüberschreitendes cT4-Rektumkarzinom vermutet. Eine CRM-Positivität wurde in 57,1\% $(n=101)$ und eine Metastasierung in intramesorektalen Lymphknoten in 89,8\% $(n=159)$ der Fälle diagnostiziert. Klinisch fortgeschrittene Tumorstadien $\geq$ cUICC-III lagen somit bei $96,1 \%$ ( $n=170)$ der Patienten vor, sodass für die Studienpopulation von einer ungünstigen Malignomsituation ausgegangen werden musste. Nach der präoperativen MMT zeigte sich im klinischen Re-Staging (yc) eine veränderte Verteilung in den UICC-Tumorstadien. Es wurden in 2,8\% $(n=5)$ der Patienten yUICC-0, in $12,4 \%(n=22)$ yUICC-I, in $27,7 \%(n=49)$ yUICC-II, in $56,5 \%$ $(n=100)$ yUICC-III und in $0,6 \%(n=1)$ yUICC-IV Stadien diagnostiziert. Die auffällig hohe Anzahl an Patienten $(n=76)$ mit < yUICC-III Stadien konnte bereits als Zeichen eines deutlichen Tumoransprechens auf die Behandlung gewertet werden. Neben der Anzahl an ycUICC-0/-I Stadien ( $n=27)$ erhöhte sich auch die Anzahl der ycUICC-II Fälle von $n=7$ auf $n=49$. 


\begin{tabular}{|c|c|c|c|c|c|c|c|c|}
\hline & \multicolumn{2}{|c|}{ Gesamt } & \multicolumn{2}{|c|}{$\begin{array}{c}\text { Kohorte A } \\
\text { 5-FU }\end{array}$} & \multicolumn{2}{|c|}{$\begin{array}{l}\text { Kohorte B } \\
\text { 5-FU + OX }\end{array}$} & \multicolumn{2}{|c|}{$\begin{array}{c}\text { Kohorte C } \\
5-\mathrm{FU}+\mathrm{OX}+\mathrm{mFOLFOX} \\
\end{array}$} \\
\hline \multicolumn{9}{|l|}{ Altersstruktur ${ }^{*}$} \\
\hline Median & 63 & & 64 & & 63 & & 62 & \\
\hline Min.-Max. & $20-82$ & & $35-82$ & & $35-81$ & & $20-74$ & \\
\hline \multicolumn{9}{|c|}{ Patientenverteilung } \\
\hline & $\mathrm{n}$ & $\%$ & $\mathrm{n}$ & $\%$ & $\mathrm{n}$ & $\%$ & $\mathrm{n}$ & $\%$ \\
\hline Kohorte & 177 & 100,0 & 64 & 36,2 & 63 & 35,6 & 50 & 28,2 \\
\hline \multicolumn{9}{|l|}{ Alter (Median)** } \\
\hline$\leq 63$ Jahre & 93 & 52,5 & 32 & 50,0 & 32 & 50,8 & 29 & 58,0 \\
\hline > 63 Jahre & 84 & 47,5 & 32 & 50,0 & 31 & 49,2 & 21 & 42,0 \\
\hline \multicolumn{9}{|l|}{ 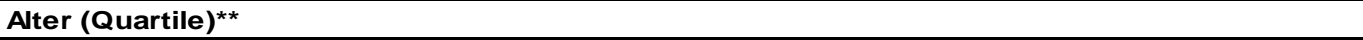 } \\
\hline$\leq 54$ Jahre & 45 & 25,4 & 12 & 18,8 & 18 & 28,6 & 15 & 30,0 \\
\hline$>54 \leq 63$ Jahre & 48 & 27,1 & 20 & 31,3 & 14 & 22,2 & 14 & 28,0 \\
\hline$>63 \leq 69$ Jahre & 40 & 22,6 & 11 & 17,2 & 15 & 23,8 & 14 & 28,0 \\
\hline$>69$ Jahre & 44 & 24,9 & 21 & 32,8 & 16 & 25,4 & 7 & 14,0 \\
\hline \multicolumn{9}{|l|}{ Gender } \\
\hline männlich & 122 & 68,9 & 46 & 71,9 & 39 & 61,9 & 37 & 74,0 \\
\hline weiblich & 55 & 31,1 & 18 & 28,1 & 24 & 38,1 & 13 & 26,0 \\
\hline \multicolumn{9}{|c|}{ Staging (klinisch, c) } \\
\hline \multicolumn{9}{|c|}{ Tumorlokalisation } \\
\hline$\leq 6 \mathrm{~cm}$ & 94 & 53,1 & 34 & 53,1 & 35 & 55,6 & 25 & 50,0 \\
\hline$>6 \mathrm{~cm}-\leq 12 \mathrm{~cm}$ & 83 & 46,9 & 30 & 46,9 & 28 & 44,4 & 25 & 50,0 \\
\hline$>12 \mathrm{~cm}$ & - & - & - & - & - & - & - & - \\
\hline \multicolumn{9}{|l|}{ Tumor (T)-Status } \\
\hline cT2 & 6 & 3,4 & 3 & 4,7 & 3 & 4,8 & - & - \\
\hline cT3 & 127 & 71,8 & 45 & 70,3 & 45 & 71,4 & 37 & 74,0 \\
\hline cT4 & 44 & 24,9 & 16 & 25,0 & 15 & 23,8 & 13 & 26,0 \\
\hline \multicolumn{9}{|l|}{ Nodal (N)-Status } \\
\hline cNO & 18 & 10,2 & 10 & 15,6 & 7 & 11,1 & 1 & 2,0 \\
\hline cN1 & 121 & 68,4 & 54 & 84,4 & 56 & 88,9 & 11 & 22,0 \\
\hline $\mathrm{cN} 2$ & 38 & 21,5 & - & - & - & - & 38 & 76,0 \\
\hline \multicolumn{9}{|c|}{ UICC-Tumorstadien } \\
\hline cUICC-0 & - & - & - & - & - & - & - & - \\
\hline cUICC-I & - & - & - & - & - & - & - & - \\
\hline cUICC-II & 7 & 4,0 & 5 & 7,8 & 1 & 1,6 & 1 & 2,0 \\
\hline cUICC-III & 161 & 91,0 & 56 & 87,5 & 59 & 93,7 & 46 & 92,0 \\
\hline cUICC-IV*** & 9 & 5,1 & 3 & 4,7 & 3 & 4,8 & 3 & 6,0 \\
\hline \multicolumn{9}{|l|}{ cCRM $^{\star \star \star \star}$} \\
\hline negativ ( $\geq 2 \mathrm{~mm}$ ) & 76 & 42,9 & 37 & 57,8 & 34 & 54,0 & 5 & 10,0 \\
\hline positiv (<2 mm) & 101 & 57,1 & 27 & 42,2 & 29 & 46,0 & 45 & 90,0 \\
\hline \multicolumn{9}{|c|}{ Präoperatives klinisches RE-Staging nach erfolgter Multimodaltherapie (yc) } \\
\hline \multicolumn{9}{|c|}{ UICC-Tumorstadien } \\
\hline ycUICC-0 & 5 & 2,8 & 1 & 1,6 & 3 & 4,8 & 1 & 2,0 \\
\hline ycUICC-I & 22 & 12,4 & 8 & 12,5 & 8 & 12,7 & 6 & 12,0 \\
\hline ycUICC-II & 49 & 27,7 & 16 & 25,0 & 16 & 25,4 & 17 & 34,0 \\
\hline ycUICC-III & 100 & 56,5 & 39 & 60,9 & 36 & 57,1 & 25 & 50,0 \\
\hline ycUICC-IV*** & 1 & 0,6 & - & - & - & - & 1 & 2,0 \\
\hline
\end{tabular}

\section{Tabelle 12: Prätherapeutisches Staging und Re-Staging}

FOLFOX: modifizierte (m) FOLFOX-CTx bestehend aus Folinsäure (FS), 5-Fluorouracil (5-FU) und Oxaliplatin (OX); Kohorte A: präoperative CRT mit 5-FU; Kohorte B: präoperative CRT und 5-FU + OX; Kohorte C: präoperative CRT und 5-FU + OX sowie drei Applikationen mFOLFOX; * Median und Quartile für die Gesamtpopulation ( $n=177)$; ${ }^{*} \mathrm{CUICC}$ IV und ycUICC-IV Stadium: Patienten mit Fernmetastasen (FM); ${ }^{* *}{ }^{*} \mathrm{CCRM}$ : zirkumferentieller Resektionsrand 


\subsection{Präoperative MMT - Dosisapplikation und Toxizität}

Von den 177 Studienteilnehmern konnten drei (1,7 \%) Patienten die geplante RTDosis nicht erhalten (Tabelle A2). Bei zwei dieser drei Patienten wurde die begleitende CTx aufgrund der Entscheidung des behandelnden Strahlentherapeuten unterbrochen. Im ersten Fall (Patient H170) wurde die OX-Dosis aufgrund von Übelkeit, Erbrechen und Diarrhoe (CTC-Grad 3) während der CRT im ersten Zyklus auf $50 \%$ reduziert. Im zweiten CTx-Zyklus wurde OX bei zunehmender gastrointestinaler Schmerzsymptomatik ausgesetzt. Die adCTx konnte fristgerecht gestartet werden, jedoch wurde die OX-Dosis auf $50 \%$ reduziert aufgrund einer prolongierten Strahlenenteritis (CTC-Grad 3) und einer PNP (CTC-Grad 2). Im zweiten Fall (H177) wurde ebenfalls aufgrund von Übelkeit, Erbrechen und Diarrhoe (CTC-Grad 3) die OX-Gabe im ersten Zyklus auf $50 \%$ reduziert und der zweite Zyklus CTx ausgesetzt. Postoperativ konnte die adCTx mit vier Zyklen FOLFOX problemlos verabreicht werden. Es waren in der Kohorte $A$ bei $1,6 \%(n=1$, Patient H97), in der Kohorte B bei 12,7\% ( $n=8$, Tabelle A11.1) und in der Kohorte $C$ bei $18,0 \%(n=9$, Tabelle A3) der Patienten Dosisreduktionen vorgenommen worden. Gemessen an der Summe aller RT-Applikationen/CTx-Zyklen $(n=1.153)$ betrug die Rate an Dosisreduktionen lediglich 12,2 \% ( $n=141$, Tabelle 23).

\subsection{Operationsverfahren und TME-Qualität}

Nach dem klinischen Re-Staging erfolgte innerhalb von zwei Wochen eine tiefe anteriore Rektumresektion (TARR) mit TME bei 126 (71,2\%) Patienten. Bei 51 $(28,8 \%)$ Patienten wurde eine abdominoperineale Rektumexstirpation (APR) mit TME durchgeführt. Aufgrund sehr weit fortgeschrittener Karzinome im unteren Rektumdrittel war bei 19 Patienten (36,5\%) eine erweiterte, zylindrische APR (eAPR) notwendig. Dies betraf vor allem Patienten der Kohorte C. In dieser Kohorte betrug der APR-Anteil insgesamt $44 \%$ infolge der Tumorlokalisation.

Die perioperative Kontrolle zeigte in 79,7\% $(n=141)$ der Rektumresektate eine gute TME-Qualität. In 17,5\% ( $n=31)$ wurde die TME-Qualität einschließlich des extraperitonealen und perinealen Weichgewebes als moderat und in 2,8 \% ( $n=5)$ als schlecht bewertet (Tabelle 13). 


\begin{tabular}{|c|c|c|c|c|c|c|c|c|}
\hline & \multicolumn{2}{|c|}{ Gesamt } & \multicolumn{2}{|c|}{$\begin{array}{c}\text { Kohorte A } \\
\text { 5-FU }\end{array}$} & \multicolumn{2}{|c|}{$\begin{array}{l}\text { Kohorte B } \\
5-F U+O X\end{array}$} & \multicolumn{2}{|c|}{$\begin{array}{c}\text { Kohorte C } \\
5-\mathrm{FU}+\mathrm{OX}+\mathrm{mFOLFOX}\end{array}$} \\
\hline & $\mathrm{n}$ & $\%$ & $\mathrm{n}$ & $\%$ & $\mathrm{n}$ & $\%$ & $\mathrm{n}$ & $\%$ \\
\hline$\Sigma$ Patienten & 177 & 100,0 & 64 & 100,0 & 63 & 100,0 & 50 & 100,0 \\
\hline \multicolumn{9}{|c|}{ OP-Verfahren mit TME } \\
\hline TARR & 126 & 71,2 & 46 & 71,9 & 52 & 82,5 & 28 & 56,0 \\
\hline APR & 51 & 28,8 & 18 & 28,1 & 11 & 17,5 & 22 & 44,0 \\
\hline \multicolumn{9}{|c|}{ Resektatqualität I* } \\
\hline gut & 141 & 79,7 & 49 & 76,6 & 52 & 82,5 & 40 & 80,0 \\
\hline moderat & 31 & 17,5 & 11 & 17,2 & 11 & 17,5 & 9 & 18,0 \\
\hline schlecht & 5 & 2,8 & 4 & 6,3 & - & - & 1 & 2,0 \\
\hline \multicolumn{9}{|c|}{ Resektatqualität II** } \\
\hline gut & 139 & 78,5 & 45 & 70,3 & 49 & 77,8 & 45 & 90,0 \\
\hline moderat & 32 & 18,1 & 15 & 23,4 & 13 & 20,6 & 4 & 8,0 \\
\hline schlecht & 6 & 3,4 & 4 & 6,3 & 1 & 1,6 & 1 & 2,0 \\
\hline
\end{tabular}

Tabelle 13: Operationsverfahren und Beurteilung der TME-Qualität

Nach erfolgter totaler mesorektaler Exzision (TME) wurde die Integrität der Resektatoberfläche durch Chirurgen (perioperativ) und später durch Pathologen (postoperativ) nach den MERCURY-Kriterien (TNM-/UICC-Klassifikation) bewertet. TARR: tiefe anteriore Rektumresektion mit Sphinktererhalt; APR: abdominoperineale Rektumexstirpation; I*: perioperative (ex situ) Untersuchung der Qualität der chirurgischen Resektatoberfläche; II**: postoperative Untersuchung der Qualität der Resektatoberfläche durch Pathologen

Unabhängig von der perioperativen TME-Beurteilung ergab die Begutachtung durch den Pathologen in 78,5\% $(n=139)$ eine gute, in $18,1 \%(n=32)$ eine moderate und in 3,4\% $(n=6)$ eine schlechte Qualität der TME einschließlich des extraperitonealen/perinealen Weichgewebes. Im Gruppenvergleich war die Resektatqualität in Kohorte $\mathrm{C}$ trotz ihres großen APR-Anteils von den Pathologen am höchsten bewertet worden. Offensichtlich hatten Chirurgen die Unversehrtheit des perinealen Weichgewebes (18,0 \% moderate Resektat-Qualität versus 8,0 \%, Tabelle 13) kritischer beurteilt. 


\subsection{Postoperatives Staging}

\subsubsection{Postoperatives pathologisches Staging}

Bei 171 (96,6 \%) Patienten wurde ein R0-Status bestätigt und bei 15 (8,5 \%) Rektumresektaten lag nach der MMT noch eine ypCRM-Positivität (CRM $<2 \mathrm{~mm}$, nach Nagtegaal et al. 2005) vor (Tabelle 14). Der Anteil an Resektaten mit einem ypCRM $<1 \mathrm{~mm}$ betrug 5,1\% ( $=9)$. In 54 (30,5\%) Rektumresektaten wurden eine ypL-/V-/Pn-Positivität und in 59 (33,3 \%) Fällen residuelle ypG3-Status diagnostiziert. Sowohl die ypL-/V-/Pn-Positivität als auch die verbliebene ypG3Differenzierung (Anhang 6.5.3) sind als unabhängige, prognostisch ungünstige Parameter einzustufen.

Unter Anwendung institutionell etablierter Methoden zur verbesserten LKAufarbeitung (Scheel et al. 2015; Gehoff et al. 2012) wurden in den 177 Rektumresektaten insgesamt 4.481 Lymphknoten untersucht. Die LK-Ausbeute betrug 22 LK im Median. Pro Resektat wurden im Mittel $25 \pm 14$ LK (Min.-Max.: 7 bis 81 LK) bewertet. Insgesamt konnten 184 LKM bei 54 (30,5\%) Patienten nachgewiesen werden. Im Median wurden 2 LKM entdeckt (Mittelwert: $3 \pm 4$ LKM pro Rektumresektat, Min.-Max.: 1 bis 22 LKM). Die Ratio zwischen der Gesamtzahl an LMK zu untersuchten LK betrug 0,041. Das Verhältnis von LKM zur LK-Zahl pro Resektat lag im Median bei 0,075 und variierte von 0,02 bis 1,0.

Nach der präoperativen CRT +/- mFOLFOX wurden als Tumorstadien 14,7\% ( $n=26)$ ypUICC-0, 23,1\% $(n=41)$ ypUICC-I, in $27,7 \%(n=49)$ ypUICC-II, $24,9 \%(n=44)$ ypUICC-III und 9,6\% $(n=17)$ ypUICC-IV Stadien diagnostiziert (Tabelle 14). 


\begin{tabular}{|c|c|c|c|c|c|c|c|c|}
\hline & \multicolumn{2}{|c|}{ Gesamt } & \multicolumn{2}{|c|}{$\begin{array}{c}\text { Kohorte A } \\
5-F U\end{array}$} & \multicolumn{2}{|c|}{$\begin{array}{l}\text { Kohorte B } \\
5 \text {-FU + OX }\end{array}$} & \multicolumn{2}{|c|}{$\begin{array}{c}\text { Kohorte C } \\
5-\mathrm{FU}+\mathrm{OX}+\mathrm{mFOLFOX}\end{array}$} \\
\hline & $\mathrm{n}$ & $\%$ & \begin{tabular}{l|l}
$\mathrm{n}$ & $\mathrm{a}$ \\
\end{tabular} & $\%$ & \begin{tabular}{l|l}
$n$ &
\end{tabular} & $\%$ & \begin{tabular}{l|l}
$\mathrm{n}$ & \\
\end{tabular} & $\%$ \\
\hline$\Sigma$ Patienten & 177 & 100,0 & 64 & 100,0 & 63 & 100,0 & 50 & 100,0 \\
\hline \multicolumn{9}{|l|}{ Residualstatus* } \\
\hline Ro & 171 & 96,6 & 62 & 96,9 & 62 & 98,4 & 47 & 94,0 \\
\hline R1 & 5 & 2,8 & 1 & 1,6 & 1 & 1,6 & 3 & 6,0 \\
\hline R2 & 1 & 0,6 & 1 & 1,6 & - & - & - & - \\
\hline \multicolumn{9}{|l|}{ ypCRM $^{\star \star}$} \\
\hline negativ ( $\geq 2 \mathrm{~mm}$ ) & 162 & 91,5 & 59 & 92,2 & 57 & 90,5 & 46 & 92,0 \\
\hline positiv $(<2 \mathrm{~mm})$ & 15 & 8,5 & 5 & 7,8 & 6 & 9,5 & 4 & 8,0 \\
\hline \multicolumn{9}{|l|}{ ypL-/V-/Pn-Status ${ }^{\star \star \star}$} \\
\hline positiv & 54 & 30,5 & 22 & 34,4 & 16 & 25,4 & 16 & 32,0 \\
\hline negativ & 123 & 69,5 & 42 & 65,6 & 47 & 74,6 & 34 & 68,0 \\
\hline \multicolumn{9}{|l|}{ ypTumor (T)-Status } \\
\hline урт0 & 29 & 16,4 & 9 & 14,1 & 9 & 14,3 & 11 & 22,0 \\
\hline ypT1 & 13 & 7,3 & 4 & 6,2 & 8 & 12,7 & 1 & 2,0 \\
\hline урТ2 & 38 & 21,5 & 16 & 25,0 & 11 & 17,5 & 11 & 22,0 \\
\hline урТ3 & 85 & 48,0 & 32 & 50,0 & 32 & 50,8 & 21 & 42,0 \\
\hline урт3а $(<1 \mathrm{~mm})$ & 27 & 15,3 & 8 & 12,5 & 9 & 14,3 & 10 & 20,0 \\
\hline урТзь (1-5 mm) & 32 & 18,1 & 14 & 21,9 & 13 & 20,6 & 5 & 10,0 \\
\hline урТЗс (> 5-15 mm) & 21 & 11,9 & 7 & 10,9 & 8 & 12,7 & 6 & 12,0 \\
\hline урТЗd (> 15 mm) & 5 & 2,8 & 3 & 4,7 & 2 & 3,2 & - & - \\
\hline урT4 & 12 & 6,8 & 3 & 4,7 & 3 & 4,8 & 6 & 12,0 \\
\hline урT4a & 3 & 1,7 & 2 & 3,1 & 1 & 1,6 & - & - \\
\hline урT4b & 9 & 5,1 & 1 & 1,6 & 2 & 3,2 & 6 & 12,0 \\
\hline \multicolumn{9}{|l|}{ ypNodal (N)-Status } \\
\hline ypNO & 123 & 69,5 & 42 & 65,5 & 47 & 74,6 & 34 & 68,0 \\
\hline ypN1 & 38 & 21,5 & 15 & 23,5 & 13 & 20,6 & 10 & 20,0 \\
\hline ypN1a (1 LNM) & 23 & 13,0 & 7 & 10,9 & 11 & 17,5 & 5 & 10,0 \\
\hline ypN1b (2-3 LNM) & 14 & 7,9 & 8 & 12,5 & 2 & 3,2 & 4 & 8,0 \\
\hline ypN1c (Satellit) & 1 & 0,6 & - & - & - & - & 1 & 2,0 \\
\hline ypN2 & 16 & 9,0 & 7 & 10,9 & 3 & 4,8 & 6 & 12,0 \\
\hline ypN2a & 8 & 4,5 & 3 & 4,7 & 2 & 3,2 & 3 & 6,0 \\
\hline ypN2b & 8 & 4,5 & 4 & 6,3 & 1 & 1,6 & 3 & 6,0 \\
\hline \multicolumn{9}{|l|}{ ypUICC-Tumorstadien } \\
\hline ypUICC-0 & 26 & 14,7 & 9 & 14,1 & 9 & 14,3 & 8 & 16,0 \\
\hline ypUICC-I & 41 & 23,1 & 14 & 21,8 & 17 & 27,1 & 10 & 19,9 \\
\hline ypUICC-II & 49 & 27,7 & 16 & 25,0 & 19 & 30,2 & 14 & 28,0 \\
\hline ypUICC-Ila & 45 & 25,4 & 16 & 25,0 & 17 & 27,0 & 12 & 24,0 \\
\hline ypUICC-llb & 3 & 1,7 & - & - & & 3,2 & 1 & 2,0 \\
\hline ypUICC-Ilc & 1 & 0,6 & - & - & - & - & 1 & 2,0 \\
\hline ypUICC-III & 44 & 24,9 & 19 & 29,7 & 12 & 19,0 & 13 & 26,0 \\
\hline ypUICC-IIla & 9 & 5,1 & 4 & 6,3 & 2 & 3,2 & 3 & 6,0 \\
\hline ypUICC-IIIIb & 23 & 13,0 & 9 & 14,1 & 10 & 15,9 & 4 & 8,0 \\
\hline ypUICC-IIIc & 12 & 6,8 & 6 & 9,4 & - & - & 6 & 12,0 \\
\hline ypUICC-IV**** & 17 & 9,6 & 6 & 9,4 & 6 & 9,5 & 5 & 10,0 \\
\hline ypUICC-IVa & 10 & 5,6 & 4 & 6,3 & 5 & 7,9 & 1 & 2,0 \\
\hline ypUICC-IVb & 7 & 4,0 & 2 & 3,1 & 1 & 1,6 & 4 & 8,0 \\
\hline
\end{tabular}

Tabelle 14: Pathologisches Staging und Tumor-Klassifikation

${ }^{*} \mathrm{R}$ : postoperativer lokoregionärer Residualstatus; ${ }^{* *} y \mathrm{pCRM}$ : histopathologisch ermittelter zirkumferentieller Resektionsrand; ${ }^{* * *} y \mathrm{pL}-/ \mathrm{V}$-/Pn-Positivität: residuelle Karzinominfiltration in Lymphgefäßen, Venen und/oder Perineuralscheiden; ${ }^{* * * *}$ ypUICC-IV Stadium: die komplette Resektion der FM war beim Staging angenommen worden. Grau: Substadien 


\subsubsection{Therapie-induzierte Tumorremission}

Die präoperative MMT führte in 16,4 \% $(n=29)$ der Patienten zu einer vollständigen Remission (CR, TRG 4, ypT0-Status) des Rektumkarzinoms (Anhang 6.5.4). Eine Tumorregression der TRG 3 und TRG 2 traten in 76,8\% $(n=139)$ und eine TRG 1 in 6,8\% $(n=12)$ ein. Insgesamt wurden bei 97 (54,8\%) Patienten ein gutes Therapieansprechen des Rektumkarzinoms (GR, good response; TRG 3/ TRG 4) und bei 80 Patienten (45,2\%) ein partielles Ansprechen (PR, partial response; TRG 1/TRG 2) nachgewiesen (Tabelle 15).

\begin{tabular}{|c|c|c|c|c|c|c|c|c|}
\hline & \multicolumn{2}{|c|}{ Gesamt } & \multicolumn{2}{|c|}{$\begin{array}{c}\text { Kohorte A } \\
\text { 5-FU }\end{array}$} & \multicolumn{2}{|c|}{$\begin{array}{l}\text { Kohorte B } \\
\text { 5-FU + OX }\end{array}$} & \multicolumn{2}{|c|}{$\begin{array}{c}\text { Kohorte C } \\
5-\mathrm{FU}+\mathrm{OX}+\mathrm{mFOLFOX}\end{array}$} \\
\hline & $\mathrm{n}$ & $\%$ & $\mathrm{n}$ & $\%$ & $\mathrm{n}$ & $\%$ & $\mathrm{n}$ & $\%$ \\
\hline$\Sigma$ Patienten & 177 & & 64 & & 63 & & 50 & \\
\hline \multicolumn{9}{|l|}{ Tumorlängsausdehnung* } \\
\hline$\leq 25 \mathrm{~mm}$ & 97 & 54,8 & 30 & 46,9 & 37 & 58,7 & 30 & 60,0 \\
\hline$>25 \mathrm{~mm}$ & 80 & 45,2 & 34 & 53,1 & 26 & 41,3 & 20 & 40,0 \\
\hline \multicolumn{9}{|l|}{ Tumor-Regressiongrade $^{\star \star}$} \\
\hline ypTRG 4 & 29 & 16,4 & 9 & 14,1 & 9 & 14,3 & 11 & 22,0 \\
\hline ypTRG 3 & 68 & 38,4 & 28 & 43,7 & 29 & 46,0 & 11 & 22,0 \\
\hline ypTRG 2 & 68 & 38,4 & 22 & 34,4 & 22 & 34,9 & 24 & 48,0 \\
\hline ypTRG 1 & 12 & 6,8 & 5 & 7,8 & 3 & 4,8 & 4 & 8,0 \\
\hline yPTRG 0 & - & - & - & - & - & - & - & - \\
\hline \multicolumn{9}{|c|}{ Ansprechen auf präoperative $\mathrm{CRT}^{\star \star \star}$} \\
\hline gut (TRG 3, TRG 4; GR) & 97 & 54,8 & 37 & 57,8 & 38 & 60,3 & 22 & 44,0 \\
\hline partiell (TRG 1, TRG 2; PR) & 80 & 45,2 & 27 & 42,2 & 25 & 39,7 & 28 & 56,0 \\
\hline \multicolumn{9}{|l|}{ ypTRG in $\%$ an Fibrose ${ }^{\star \star * *}$} \\
\hline$<20 \%$ & 3 & 1,7 & 1 & 1,6 & 1 & 1,6 & 1 & 2,0 \\
\hline $20-39 \%$ & 7 & 4,0 & 4 & 6,2 & 1 & 1,6 & 2 & 4,0 \\
\hline $40-49 \%$ & 2 & 1,1 & 1 & 1,6 & 1 & 1,6 & - & - \\
\hline $50-59 \%$ & 16 & 9,0 & 6 & 9,4 & 4 & 6,3 & 6 & 12,0 \\
\hline $60-69 \%$ & 21 & 11,9 & 7 & 10,9 & 5 & 7,9 & 9 & 18,0 \\
\hline 70-79 \% & 22 & 12,4 & 8 & 12,5 & 12 & 19,1 & 2 & 4,0 \\
\hline $80-89 \%$ & 27 & 15,3 & 8 & 12,5 & 9 & 14,3 & 10 & 20,0 \\
\hline $90-99 \%$ & 50 & 28,3 & 20 & 31,2 & 21 & 33,3 & 9 & 18,0 \\
\hline $100 \%$ & 29 & 16,4 & 9 & 14,1 & 9 & 14,3 & 11 & 22,0 \\
\hline
\end{tabular}

Tabelle 15: Residuelle Tumorlängsausdehnung (ypTE) und Tumorregression

yp: nach präoperativer MMT pathologischer Befund; * postoperative Tumorlängsausdehnung (ypTE) nach MMT (Median: $25 \mathrm{~mm}$ ); ** Tumor-Regressionsgrade (ypTRG): 5stufiges Tumor-Regressionsgrading-System (Dworak et al. 1997); ${ }^{\star \star \star}$ Ansprechen auf präoperativeCRT: gutes Ansprechen (GR, good response) umfasst alle Fälle mit ypTRG 3 und ypTRG 4; partielles Ansprechen (PR, partial response) gilt für alle Fälle mit ypTRG 1 und ypTRG 2; ${ }^{* * *}$ ypTRG in \% an Fibrose: Angabe der Therapieinduzierten Tumorfibrose in Prozent 
Darüber hinaus konnte nach der präoperativen CRT eine Schrumpfung der Tumorlängsausdehnung (ypTE) auf $25 \mathrm{~mm}$ (Median und Mittelwert: 25,0 mm, Standardabweichung $\pm 18 \mathrm{~mm}$, Min.-Max.: 0-115 mm) festgestellt werden. Bezogen auf diesen Medianwert betrug die residuelle Tumorausdehnung bei 54,8\% $(\mathrm{n}=97)$ der Patienten $\leq 25 \mathrm{~mm}$ und bei 23,3\% $(\mathrm{n}=41)$ der Patienten $\leq 10 \mathrm{~mm}$. Die im Staging diagnostizierte CRM-Positivität von $57,1 \%$ war nach der präopereativen CRT +/- mFOLFOX auf 8,5\% ( $n=15)$ gesunken (Tabellen 12, 14 und 15).

\subsection{Präoperative Multimodaltherapie - Toxizitätsgrade}

In den nachfolgenden Tabellen (Tabellen 16 bis 22, im Anhang Tabelle A5 und A6) sind die während der präoperativen CRT +/- mFOLFOX-CTx aufgetretenen Nebenwirkungen (NWK) nach Symptomen und Toxizitätsgraden der NCl-CTCAEKlassifikation (Version 4.03) ( $\mathrm{NCl} 2010$ ) dargestellt. Für alle 177 Patienten aus den Kohorten A, B und C wurden 918 CTx-bedingte Ereignisse erfasst. Davon waren $646(70,4 \%)$ Ereignisse unbedeutend (Grad 1: mit Anämie (HB-Abfall), Zystitis, Hauttoxizität, Strahlenproktitis) und 232 (25\%) Ereignisse hatten eine z. T. geringe klinische Relevanz (Grad 2: Hauttoxizität, Strahlenproktitis, intestinale NW). Neurologische Nebenwirkungen traten in 41 Fällen mit CTC-Grad 1 und bei 6 Patienten mit CTC-Grad 2 (z. B. PNP) auf. Insgesamt wurden 40 Ereignisse mit Grad 3 und/oder Grad 4 Toxizitäten festgestellt. Diese führten zu einer Dosisreduktion der CTx, wie z. B. in zwei Fällen mit einer schweren Zystitis (CTC-Grad 4, Tabelle A5). Unter den CTC-Grad 3 Toxizitäten fanden sich zu $25 \%$ intestinale Nebenwirkungen (10 Ereignisse, Tabelle A5). Jeweils 5x wurden Hauttoxizitäten und gastrale Nebenwirkungen im CTC-Grad 3 aufgeführt. Eine Strahlenproktitis CTC-Grad 3 wurde 4x erfasst und die verbleibenden 15 Fälle mit CTC-Grad $3 \mathrm{Ne}$ benwirkungen verteilten sich auf Ereignisse wie Fieber, Infektionen, allergische Reaktionen sowie sonstige kurzzeitig pathologisch veränderte Laborwerte. 


\subsubsection{Präoperative CRT - Toxizitätsgrade in den Kohorten A, B und C}

In den Tabellen 16 bis 18 sind die während der präoperativen CRT aufgetretenen Toxizitäten aufgelistet. Es ließen sich keine signifikanten Unterschiede im Auftreten klinisch relevanter Toxizitätsgrade feststellen.

\begin{tabular}{|c|c|c|c|c|c|c|c|c|c|}
\hline \multirow{2}{*}{$\begin{array}{l}n=64 \text { Patienten } \\
\text { Nebenwirkungen } \\
\text { in CTC-Grade } \geq 1\end{array}$} & \multicolumn{2}{|c|}{ Gesamt } & \multicolumn{7}{|c|}{$\begin{array}{c}\text { Kohorte A } \\
\text { Toxizitätsgrade (NCI-CTCAE Version 4.03) }\end{array}$} \\
\hline & Ereignisse* & $\%$ & 1 & 2 & 3 & 4 & 5 & $\geq 2$ & $\geq 3$ \\
\hline \multicolumn{10}{|l|}{ Unter präop. CRT } \\
\hline Geschmacksinn/-irritation & - & - & - & - & - & - & - & - & - \\
\hline Schmerz & 2 & 0,9 & 1 & 1 & - & - & - & 1 & - \\
\hline Fatigue & 1 & 0,4 & 1 & - & - & - & - & - & - \\
\hline Hämoglobin & 37 & 16,5 & 28 & 7 & 2 & - & - & 9 & 2 \\
\hline Leukozyten & 13 & 5,8 & 8 & 4 & 1 & - & - & 5 & 1 \\
\hline Thrombozyten & 10 & 4,5 & 10 & - & - & - & - & - & - \\
\hline konstitutionelle Symptome ** & - & - & - & - & - & - & - & - & - \\
\hline Kreatinin & - & - & - & - & - & - & - & - & - \\
\hline Haut & 51 & 22,8 & 28 & 22 & 1 & - & - & 23 & 1 \\
\hline Stomatitis/Mukositis & - & - & - & - & - & - & - & - & - \\
\hline Zystitis & 21 & 9,4 & 16 & 5 & - & - & - & 5 & - \\
\hline Proktitis & 51 & 22,8 & 23 & 28 & - & - & - & 28 & - \\
\hline intestinale NWK & 28 & 12,5 & 21 & 7 & - & - & - & 7 & - \\
\hline gastrale NWK & 8 & 3,6 & 4 & 4 & - & - & - & 4 & - \\
\hline renale NWK & - & - & - & - & - & - & - & - & - \\
\hline neurologische NWK & - & - & - & - & - & - & - & - & - \\
\hline kardiale NWK & - & - & - & - & - & - & - & - & - \\
\hline Fieber/Infektion & 2 & 0,9 & - & 1 & 1 & - & - & 2 & 1 \\
\hline Alopezie & - & - & - & - & - & - & - & - & - \\
\hline Allergische Reaktion & - & - & - & - & - & - & - & - & - \\
\hline$A Z$ & - & - & - & - & - & - & - & - & - \\
\hline Dyspnoe & - & - & - & - & - & - & - & - & - \\
\hline IL-6-Syndrom & - & - & - & - & - & - & - & - & - \\
\hline Neutrophile & - & - & - & - & - & - & - & - & - \\
\hline Thrombose (TVT) & - & - & - & - & - & - & - & - & - \\
\hline Hepar/Transaminasen, Bilirubin & - & - & - & - & - & - & - & - & - \\
\hline Hand-Fuß-Syndrom & - & - & - & - & - & - & - & - & - \\
\hline Sonstige & - & - & - & - & - & - & - & - & - \\
\hline Summe & 224 & 100,0 & 140 & 79 & 5 & - & - & 84 & 5 \\
\hline
\end{tabular}

Tabelle 16: Akute Nebenwirkungen präoperativer MMT (Kohorte A)

*Ereignisse: jedes Ereignis einer akuten hämatologischen und/oder Organ-Toxizität wurde mit dem höchsten CTC-Grad dokumentiert, die während und drei Wochen nach der präoperativen CRT bei Patienten der Kohorte $A(n=64)$ aufgetreten waren; **Ernährungszustand und Kognition wurden als "konstitutionelle Symptome“ zusammengefasst. Farblich hervorgehoben sind in der Tabelle die prozentual häufigsten Symptome; zudem werden klinisch relevante Toxizitäten aufgeführt ( $\geq$ Grad 3 bzw. bei Leukozyten $\geq$ Grad 2), da sie häufig als Begründung für Dosisreduktionen dienten. 


\begin{tabular}{|c|c|c|c|c|c|c|c|c|c|}
\hline \multirow{2}{*}{$\begin{array}{l}\mathrm{n}=63 \text { Patienten } \\
\text { Nebenwirkungen } \\
\text { in CTC-Grade } \geq 1\end{array}$} & \multicolumn{2}{|c|}{ Gesamt } & \multicolumn{7}{|c|}{$\begin{array}{c}\text { Kohorte B } \\
\text { Toxizitätsgrade (NCI-CTCAE Version 4.03) }\end{array}$} \\
\hline & \begin{tabular}{|l|} 
Ereignisse \\
\end{tabular} & $\%$ & 1 & 2 & 3 & 4 & 5 & $\geq 2$ & $\geq 3$ \\
\hline \multicolumn{10}{|l|}{ Unter präop. CRT } \\
\hline Geschmacksinn/-irritation & - & - & - & - & - & - & - & - & - \\
\hline Schmerz & 4 & 1,6 & 2 & 1 & 1 & - & - & 2 & 1 \\
\hline Fatigue & 2 & 0,8 & 1 & 1 & - & - & - & - & - \\
\hline Hämoglobin & 39 & 15,4 & 36 & 3 & - & - & - & 3 & - \\
\hline Leukozyten & 23 & 9,1 & 16 & 6 & 1 & - & - & 7 & 1 \\
\hline Thrombozyten & 4 & 1,6 & 4 & - & - & - & - & - & - \\
\hline konstitutionelle Symptome ** & 7 & 2,8 & 6 & 1 & - & - & - & 1 & - \\
\hline Kreatinin & - & - & - & - & - & - & - & - & - \\
\hline Haut & 37 & 14,6 & 20 & 15 & 2 & - & - & 17 & 2 \\
\hline Stomatitis/Mukositis & 2 & 0,8 & 1 & - & 1 & - & - & 1 & 1 \\
\hline Zystitis & 23 & 9,1 & 12 & 9 & 1 & 1 & - & 11 & 2 \\
\hline Proktitis & 46 & 18,2 & 27 & 19 & - & - & - & 19 & - \\
\hline intestinale NWK & 32 & 12,6 & 15 & 10 & 7 & - & & 17 & 7 \\
\hline gastrale NWK & 17 & 6,7 & 7 & 5 & 5 & - & - & 10 & 5 \\
\hline renale NWK & - & - & - & - & - & - & - & - & - \\
\hline neurologische NWK & 7 & 2,8 & 6 & 1 & - & - & - & 1 & - \\
\hline kardiale NWK & 1 & 0,4 & 1 & - & - & - & - & - & - \\
\hline Fieber/Infektion & 5 & 2,0 & 1 & 2 & 2 & - & - & 4 & 2 \\
\hline Alopezie & - & - & - & - & - & - & - & - & - \\
\hline Allergische Reaktion & 2 & 0,8 & - & 1 & 1 & - & - & 2 & 1 \\
\hline $\mathrm{AZ}$ & - & - & - & - & - & - & - & - & - \\
\hline Dyspnoe & - & - & - & - & - & - & - & - & - \\
\hline IL-6-Syndrom & 1 & 0,4 & - & 1 & - & - & - & 1 & - \\
\hline Neutrophile & - & - & - & - & - & - & - & - & - \\
\hline Thrombose (TVT) & - & - & - & - & - & - & - & - & - \\
\hline Hepar/Transaminasen, Bilirubin & - & - & - & - & - & - & - & - & - \\
\hline Hand-Fuß-Syndrom & 1 & 0,4 & - & 1 & - & - & - & 1 & - \\
\hline Sonstige & - & - & - & - & - & - & - & - & - \\
\hline Summe & 253 & 100,0 & 155 & 76 & 21 & 1 & - & 97 & 22 \\
\hline
\end{tabular}

Tabelle 17: Akute Nebenwirkungen präoperativer MMT (Kohorte B)

${ }^{*}$ Ereignisse: jedes Ereignis einer akuten hämatologischen und/oder Organ-Toxizität wurde mit dem höchsten CTC-Grad dokumentiert, die während und innerhalb von drei Wochen nach der präoperativen CRT bei Patienten der Kohorte B $(n=63)$ aufgetreten waren; **Ernährungszustand und Kognition wurden als "konstitutionelle Symptome“ zusammengefasst. Farblich hervorgehoben sind in der Tabelle die prozentual häufigsten Symptome; zudem werden klinisch relevante, höhergradige Toxizitäten mit klinischer Konsequenz aufgeführt ( $\geq$ Grad 3 bzw. bei Leukozyten $\geq$ Grad 2), da sie häufig als Begründung für Dosisreduktionen dienten.

Im Vergleich zu den Patientenkohorten A und C weist Kohorte B eine höhere Anzahl an CTC-Grad 3 Toxizitäten auf. 


\begin{tabular}{|c|c|c|c|c|c|c|c|c|c|}
\hline \multirow{2}{*}{$\begin{array}{l}\mathrm{n}=50 \text { Patienten } \\
\text { Nebenwirkungen } \\
\text { in CTC-Grade } \geq 1\end{array}$} & \multicolumn{2}{|c|}{ Gesamt } & \multicolumn{7}{|c|}{$\begin{array}{c}\text { Kohorte C } \\
\text { Toxizitätsgrade (NCI-CTCAE Version 4.03) }\end{array}$} \\
\hline & \begin{tabular}{|l|} 
Ereignisse ${ }^{*}$ \\
\end{tabular} & $\%$ & 1 & 2 & 3 & 4 & 5 & $\geq 2$ & $\geq 3$ \\
\hline \multicolumn{10}{|l|}{ Unter präop. CRT } \\
\hline Geschmacksinn/-irritation & - & - & - & - & - & - & - & - & - \\
\hline Schmerz & 4 & 2,0 & 3 & 1 & - & - & - & 1 & - \\
\hline Fatigue & - & - & - & - & - & - & - & - & - \\
\hline Hämoglobin & 29 & 14,5 & 26 & 3 & - & - & - & 3 & - \\
\hline Leukozyten & 16 & 8,0 & 12 & 4 & - & - & - & 4 & - \\
\hline Thrombozyten & 6 & 3,0 & 5 & 1 & - & - & - & 1 & - \\
\hline konstitutionelle Symptome ${ }^{\star *}$ & 1 & 0,5 & - & 1 & - & - & - & 1 & - \\
\hline Kreatinin & - & - & - & - & - & - & - & - & - \\
\hline Haut & 30 & 15,0 & 16 & 12 & 2 & - & - & 14 & 2 \\
\hline Stomatitis/Mukositis & - & - & - & - & - & - & - & - & - \\
\hline Zystitis & 24 & 12,0 & 20 & 3 & 1 & - & - & 4 & 1 \\
\hline Proktitis & 39 & 19,5 & 22 & 13 & 4 & - & - & 17 & 4 \\
\hline intestinale NWK & 29 & 14,5 & 15 & 11 & 3 & - & - & 14 & 3 \\
\hline gastrale NWK & 8 & 4,0 & 6 & 2 & - & - & - & 2 & - \\
\hline renale NWK & - & - & - & - & - & - & - & - & - \\
\hline neurologische NWK & 1 & 0,5 & - & 1 & - & - & - & 1 & - \\
\hline kardiale NWK & - & - & - & - & - & - & - & - & - \\
\hline Fieber/Infektion & 3 & 1,5 & 3 & - & - & - & - & - & - \\
\hline Alopezie & - & - & - & - & - & - & - & - & - \\
\hline Allergische Reaktion & 1 & 0,5 & 1 & - & - & - & - & - & - \\
\hline $\mathrm{AZ}$ & - & - & - & - & - & - & - & - & - \\
\hline Dyspnoe & - & - & - & - & - & - & - & - & - \\
\hline IL-6-Syndrom & - & - & - & - & - & - & - & - & - \\
\hline Neutrophile & - & - & - & - & - & - & - & - & - \\
\hline Thrombose (TVT) & - & - & - & - & - & - & - & - & - \\
\hline Hepar/Transaminasen, Bilirubin & - & - & - & - & - & - & - & - & - \\
\hline Hand-Fuß-Syndrom & - & - & - & - & - & - & - & - & - \\
\hline Sonstige & 9 & 4,5 & 3 & 4 & 2 & - & - & 6 & 2 \\
\hline Summe & 200 & 100,0 & 132 & 56 & 12 & - & - & 68 & 12 \\
\hline
\end{tabular}

Tabelle 18: Akute Nebenwirkungen unter präoperativer MMT (Kohorte C)

*Ereignisse: jedes Ereignis einer akuten hämatologischen und/oder Organ-Toxizität wurde mit dem höchsten CTC-Grad dokumentiert, die während und innerhalb von drei Wochen nach der präoperativen CRT bei Patienten der Kohorte $C(n=50)$ aufgetreten waren; **Ernährungszustand und Kognition wurden als "konstitutionelle Symptome“ zusammengefasst. Farblich hervorgehoben sind in der Tabelle die prozentual häufigsten Symptome; zudem werden klinisch relevante, höhergradige Toxizitäten mit klinischer Konsequenz aufgeführt ( $\geq$ Grad 3 bzw. bei Leukozyten $\geq$ Grad 2), da sie häufig als Begründung für Dosisreduktionen dienten.

\subsubsection{Präoperative CTx nach CRT - Toxizitätsgrade Kohorte C}

In Kohorte $C(n=50)$ kam es nach der Komplettierung der präoperativen CRT während der mFOLFOX-Applikationen zu 241 Ereignissen (Tabelle 19). Darunter waren 219 Ereignisse mit Grad 1, 21 Ereignisse mit Grad 2 und ein Ereignis mit Grad 3 (Leukopenie) Nebenwirkungen. Die CTx-assoziierten Organ-Toxizitäten umfassten 44 Hämoglobinabfälle, die bei 39 Patienten einen passageren CTC-Grad 1 und in 5 Fällen einen CTC-Grad 2 aufwiesen. Zudem waren 39 neu- 
rologische Ereignisse mit CTC-Grad 1 in 35 Fällen und Grad 2 Toxizitäten bei vier Patienten dokumentiert worden. Temporäre Leukopenien traten in 28 Fällen auf, davon in 23 Fällen mit Grad 1, in vier Fällen mit Grad 2 und in einem Fall mit Grad 3. Die Thrombozytenwerte waren in 21 Fällen leicht reduziert (CTC-Grad 1).

\begin{tabular}{|c|c|c|c|c|c|c|c|c|c|}
\hline \multirow{2}{*}{$\begin{array}{l}\mathrm{n}=50 \text { Patienten } \\
\text { Nebenwirkungen } \\
\text { in CTC-Grade } \geq 1\end{array}$} & \multicolumn{2}{|c|}{ Gesamt } & \multicolumn{7}{|c|}{$\begin{array}{c}\text { Kohorte C } \\
\text { Toxizitätsgrade (NCI-CTCAE Version 4.03) }\end{array}$} \\
\hline & \begin{tabular}{|l|} 
Ereignisse \\
\end{tabular} & $\%$ & 1 & 2 & 3 & 4 & 5 & $\geq 2$ & $\geq 3$ \\
\hline \multicolumn{10}{|l|}{ Unter präop. CTx nach CRT } \\
\hline Geschmacksinn/-irritation & 8 & 3,3 & 8 & - & - & - & - & - & - \\
\hline Schmerz & 11 & 4,6 & 9 & 2 & - & - & - & 2 & - \\
\hline Fatigue & 19 & 7,9 & 18 & 1 & & - & - & 1 & - \\
\hline Hämoglobin & 44 & 18,3 & 39 & 5 & - & - & - & 5 & - \\
\hline Leukozyten & 28 & 11,6 & 23 & 4 & 1 & - & - & 5 & 1 \\
\hline Thrombozyten & 21 & 8,7 & 21 & - & - & - & - & - & - \\
\hline konstitutionelle Symptome ** & 17 & 7,1 & 16 & 1 & - & - & - & 1 & - \\
\hline Kreatinin & 4 & 1,7 & 4 & - & - & - & - & - & - \\
\hline Haut & 7 & 2,9 & 6 & 1 & - & - & - & 1 & - \\
\hline Stomatitis/Mukositis & 5 & 2,1 & 5 & - & - & - & - & - & - \\
\hline Zystitis & - & - & - & - & - & - & - & - & - \\
\hline Proktitis & - & - & - & - & - & - & - & - & - \\
\hline intestinale NWK & 14 & 5,8 & 13 & 1 & & - & - & 1 & - \\
\hline gastrale NWK & 13 & 5,4 & 11 & 2 & - & - & - & 2 & - \\
\hline renale NWK & 3 & 1,2 & 3 & - & - & - & - & - & - \\
\hline neurologische NWK & 39 & 16,2 & 35 & 4 & - & - & - & 4 & - \\
\hline kardiale NWK & - & - & - & - & - & - & - & - & - \\
\hline Fieber/Infektion & 2 & 0,8 & 2 & - & - & - & - & - & - \\
\hline Alopezie & 2 & 0,8 & 2 & - & - & - & - & - & - \\
\hline Allergische Reaktion & - & - & - & - & - & - & - & - & - \\
\hline $\mathrm{AZ}$ & 2 & 0,8 & 2 & - & - & - & - & - & - \\
\hline Dyspnoe & - & - & - & - & - & - & - & - & - \\
\hline IL-6-Syndrom & - & - & - & - & - & - & - & - & - \\
\hline Neutrophile & - & - & - & - & - & - & - & - & - \\
\hline Thrombose (TVT) & - & - & - & - & - & - & - & - & - \\
\hline Hepar/Transaminasen, Bilirubin & - & - & - & - & - & - & - & - & - \\
\hline Hand-Fuß-Syndrom & - & - & - & - & - & - & - & - & - \\
\hline Sonstige & 2 & 0,8 & 2 & - & - & - & - & - & - \\
\hline Summe & 241 & 100,0 & 219 & 21 & 1 & - & - & 22 & 1 \\
\hline
\end{tabular}

Tabelle 19: Akute Nebenwirkungen präoperativer mFOLFOX-CTx (Kohorte C)

Die drei Applikationen der mFOLFOX-CTx erfolgten nach der präoperativen CRT mit 5-FU + OX; *Ereignisse: jedes Ereignis einer akuten hämatologischen und/oder OrganToxizität wurde mit dem höchsten CTC-Grad dokumentiert, die während und innerhalb von drei Wochen nach Komplettierung der präoperativen CRT bei Patienten der Kohorte $B(n=50)$ aufgetreten waren; **Ernährungszustand und Kognition wurden als „konstitutionelle Symptome“ zusammengefasst. Farblich hervorgehoben sind die prozentual häufigsten Symptome; zudem werden klinisch relevante, höhergradige Toxizitäten mit klinischer Konsequenz aufgeführt ( $\geq$ Grad 3 bzw. bei Leukozyten $\geq$ Grad 2), da sie häufig als Begründung für Dosisreduktionen dienten. 


\subsection{Postoperative additive CTx, Dosis und Toxizitäten}

Nach Abschluss der präoperativen CRT und erfolgter TME war für 127 Patienten der Kohorten $A(n=64)$ und $B(n=63)$ eine adCTx mit 5-FU oder FOLFOX geplant. Zehn Patienten erhielten postoperativ keine adCTx aufgrund der Entscheidung ihrer behandelnden Ärzte oder auf Wunsch der Patienten (Abbildung 3, Tabelle A1 im Anhang 6.1). Dies betraf fünf Patienten der Kohorte A (Patienten H1, H42, H49, H64 und H154) sowie vier Patienten der Kohorte B (Patienten H51, H70, H96 und H100). Bei einem Patienten der Kohorte B (H96, 60 Jahre, APR, ypUICC-IVb Stadium) wurde aufgrund eines R2b-Status und einer progredienten ypM1-Situation eine palliative CTx eingeleitet. Dieser Patient starb fünf Monate (OS) nach Rektumresektion am Tumorprogress. Insgesamt lehnten vier Patienten die geplante adCTx nachträglich unter Hinweis auf ihr fortgeschrittenes Alter (> 80 Jahre) und ihre bisherigen Therapieerfahrungen ab. Bei einem weiteren Patienten (H113, 69 Jahre, Tabelle A1) der Kohorte C mit R2b-Status wurde die polytope hepatische Metastasierung (ypUICC-IVb) mit drei Zyklen FOLFIRI-CTx weiter behandelt. Dieser Patient verstarb acht Monate nach OP am Malignomprogress. Bei elf Patienten hatte ein Wechsel der ursprünglich geplanten CTx stattgefunden (CONSORT-Schema, Abbildung 3).

Außerdem wurden vier Patienten der Kohorte $C$ entgegen der ursprünglichen Planung einer alleinigen Nachbeobachtung postoperativ mit einer FOLFOX-CTx (nach Kohorte B) behandelt. Im ersten Fall (H102, 51 Jahre, APR, ypUICC-IVa) mit resektabel erscheinenden synchronen bilobulären LM wurden vor der zweizeitig geplanten Hemihepatektomie (Segmente V-VIII) noch drei Einzelgaben mFOLFOX verabreicht. Bei diesem Patienten betrug das RFS 40 Monate bis zum Auftreten von pulmonalen Metastasen. Letztlich verstarb der Patient an progredienten ossären und cerebralen Metastasen in der heimatnahen spezialisierten ambulanten Palliativversorgung (SAPV) 60 Monate (OS) nach der Rektumresektion. In dem zweiten Patientenfall (H131, 63 Jahre, ypUICC-IVb, APR, Kohorte C) wurde nach der erweiterten Rektumresektion mit LM-Resektion ein R0-Status im Stadium ypUICC-IVb bestätigt. Aufgrund der histopathologisch nachgewiesenen hochgradigen Tumorregression im Primarius sowie in den LM in den Segmenten II 
(LM: 0,5 cm, ypG3) und VI (LM: 0,4 cm, ypG3) wurde postoperativ die FOLFOXTherapie in „pseudoadjuvanter" Intention mit drei Zyklen fortgesetzt. Der Patient war bis zum letzten Beobachtungszeitpunkt tumorfrei geblieben (RFS: bisher 50 Monate). Beim dritten Patienten (H123, 57 Jahre, m, APR, ypUICC-IVb, Kohorte C) mit einem R1-Status im viszeralen Peritoneum wurde auf Empfehlung des behandelnden Chirurgen und seines Verdachts auf eine R2b-Situation in situ nach dem dritten Zyklus FOLFOX eine weitere CTx mit FOLFIRI + Cetuximab (drei Zyklen) eingeleitet. Im weiteren Verlauf bestätigte sich leider eine peritoneale und lymphogene Metastasierung. Der Patient starb aufgrund eines rasanten Tumorprogresses 10 Monate nach der Rektumresektion (OS, RFS: 0 Monate). Der vierte Patient (H128, 66 Jahre, m, ypUICC-Ila, TARR, Kohorte C) wurde aufgrund eines R1- und eines positiven CRM-Status postoperativ mit drei Zyklen FOLFOX behandelt. Der Patient hatte bis zur letzten Beobachtung ein RFS von 61 Monaten.

Von insgesamt 118 Patienten, die für eine postoperative adCTx qualifiziert waren, starteten tatsächlich 52 (44,1\%) Patienten mit 5-FU und 66 (55,9\%) Patienten mit mFOLFOX (Tabelle 20). Die adCTx musste bei einem Patienten nach Abschluss von zwei Zyklen FOLFOX-CTx aufgrund von allergischen Reaktionen und kardialen Nebenwirkungen abgebrochen werden. Dieser Patient (H161, Tabelle A4) verstarb 14 Tage nach der letzten CTx-Gabe in seiner häuslichen Umgebung infolge eines kardialen Sick-Sinus-Syndroms. Bei dem Patienten war der obligatorische prätherapeutische DPD-Exon-14-Skipping-Mutationstest unauffällig gewesen. 


\begin{tabular}{|c|c|c|c|c|c|c|}
\hline & \multicolumn{2}{|c|}{ Gesamt } & \multicolumn{2}{|c|}{$\begin{array}{c}\text { Kohorte A } \\
\text { 5-FU }\end{array}$} & \multicolumn{2}{|c|}{$\begin{array}{l}\text { Kohorte B } \\
5-F U+O X\end{array}$} \\
\hline & $\mathrm{n}$ & & $\mathrm{n}$ & & $\mathrm{n}$ & \\
\hline \multicolumn{7}{|l|}{ Patienten / Therapie } \\
\hline Patienten (postop. adCTx geplant) & \multicolumn{2}{|l|}{127} & \multicolumn{2}{|l|}{64} & \multicolumn{2}{|l|}{63} \\
\hline Postop. adCTx nicht möglich & \multicolumn{2}{|l|}{8} & \multicolumn{2}{|l|}{5} & \multicolumn{2}{|l|}{3} \\
\hline Postop. palliative CTx & \multicolumn{2}{|l|}{1} & \multicolumn{2}{|l|}{-} & \multicolumn{2}{|l|}{$1^{*}$} \\
\hline $\begin{array}{l}\text { Postop. Therapiewechsel } \\
\text { zwischen den Kohorten A und B }\end{array}$ & \multicolumn{2}{|l|}{11} & \multicolumn{2}{|l|}{9} & \multicolumn{2}{|l|}{2} \\
\hline Patienten as-treated & \multicolumn{2}{|l|}{118} & \multicolumn{2}{|l|}{52} & \multicolumn{2}{|l|}{66} \\
\hline & $n$ & $\%$ & $\bar{n}$ & $\%$ & n & $\%$ \\
\hline \multicolumn{7}{|c|}{ Patienten mit tatsächlich applizierten Zyklen der adCTx } \\
\hline adCTx gestartet & 118 & 100,0 & 52 & 100,0 & 66 & $\overline{100,0}$ \\
\hline 1 Zyklus adCTx & 118 & 100,0 & 52 & 100,0 & 66 & 100,0 \\
\hline$\leq 2$ Zyklen adCTx & 116 & 98,3 & 51 & 98,1 & 65 & 98,5 \\
\hline$\leq 3$ Zyklen adCTx & 112 & 94,9 & 50 & 96,2 & 62 & 93,9 \\
\hline$\leq 4$ Zyklen adCTx & 105 & 89,0 & 49 & 94,2 & 56 & 84,8 \\
\hline \multicolumn{7}{|c|}{ Patienten mit und ohne Dosisreduktion adCTx } \\
\hline ohne Dosisreduktion & 84 & 71,2 & 46 & 88,5 & 38 & 57,6 \\
\hline mit Dosisreduktion & 34 & 28,8 & 6 & 11,5 & 28 & 42,4 \\
\hline
\end{tabular}

Tabelle 20: Postoperative Therapie in den Patientenkohorten A und B *Patient mit einem R2b- und ypUICC-IVb Status

Insgesamt erhielten alle 118 Patienten mindestens einen Zyklus der adCTx, 116 Patienten zwei (98,3\%) Zyklen, 112 (94,9\%) Patienten drei Zyklen und 105 $(89,0 \%)$ Studienteilnehmer vier Zyklen (Tabelle 20). Eine Dosisreduktion erfolgte bei $34(28,8 \%)$ Patienten. Es betraf sechs der 52 (11,5\%) Patienten unter additiver 5-FU CTx und 28 (42,4\%) Patienten unter dem FOLFOX Regime. Bei fünf $(4,2 \%)$ Patienten wurden allein die OX-Dosierungen reduziert. Darüber hinaus wurde bei zwei Patienten (1,7 \%) die OX-Gabe während der adCTx aufgrund von allergischen Reaktionen, Unwohlsein und subjektiven intolerablen neurologischen Nebenwirkungen, wie z. B. Kribbelparästhesien und Kälteempfindlichkeit in den Akren, unterbrochen bzw. abgesetzt (Kohorte B). Interessanterweise war bei keinem der 46 Patienten aus der Kohorte C (CONSORT-Schema, Abbildung 3) während der präoperativen mFOLFOX-CTx ein Therapieabbruch aufgrund von relevanten Nebenwirkungen notwendig gewesen.

Bei insgesamt drei Patienten (Tabelle A4) wurde das postoperativ geplante adCTx-Regime modifiziert. In einem Fall wurde statt vier Zyklen mit infusionalem 5-FU eine äquivalente Medikation mit Capecitabin (Xeloda®) verabreicht (Patient H63, Kohorte B). Die Umstellung der postoperativen adCTx auf die alleinige orale 
5-FU-Medikation erfolgte als Vorsichtsmaßnahme, da der Patient (H63, Kohorte B, Tabelle A4) während der präoperativen CRT allergische Reaktionen auf die Kombination von infusionalem 5-FU + OX angab. Klinisch konnten diese Angaben nicht verifiziert werden, aber aufgrund eines zusätzlichen Portinfekts erfolgte die Entscheidung zur Portexplantation und nachfolgenden Therapieumstellung. In der Bewertung der CTx, z. B. der CTx-assoziierten Nebenwirkungen, wird dieser Patient weiterhin als 5-FU Kasus geführt.

Die unter der adCTx aufgetretenen akuten Nebenwirkungen sind für alle 118 Patienten in Tabelle A6 aufgeführt. Die Toxizitäten zeigen ein heterogenes Bild. Jeweils weniger als $16 \%$ der beobachteten Ereignisse entfallen auf gastrale $\mathrm{Ne}$ benwirkungen (15,7 \%) und passagere HB-Abfälle (Anämie, 15,4\%). Aus den verbleibenden $70 \%$ der Toxizitätsereignisse lässt sich keine zukünftige Handlungsanweisung ableiten. Von 635 dokumentierten Toxizitätsereignissen entfielen $65 \%(n=413)$ auf den CTC-Grad 1 und 29,3\% $(n=186)$ auf den CTC-Grad 2. Die klinisch relevanten CTC-Grad 3 Toxizitäten $(n=29,4,6 \%)$ ergaben sich aus intestinalen Nebenwirkungen $(n=7)$, Fieber/Infektionen $(n=7)$, gastralen Begleiterscheinungen $(n=4)$ und potentiell lebensbedrohlichen Gefäßthrombosen $(n=3)$. Sechs Fälle mit CTC-Grad 4 Ereignissen zeigten keinen organbezogenen Symptomkomplex. Der einzige kardial bedingte Todesfall (CTC-Grad 5, Tabelle 22; Tabelle A4: Patient H161, postoperativer adCTx), ist aufgrund der anatomischen Normvariante nur eingeschränkt als Folge der MMT zu werten.

In den nachfolgenden Tabellen 21 und 22 sind die Toxizitätsereignisse für die Kohorten $A$ und $B$ jeweils separat dargestellt. Auffällig ist die nahezu doppelt so hohe Anzahl der Toxizitätsereignisse in der Kohorte B. Die Anzahl der klinisch relevanten $\geq$ Grad 3 Nebenwirkungen weisen dagegen nur einen geringen Unterschied auf (Kohorte $A: n=16$ und Kohorte $B: n=20$ ). Im Vergleich der höhergradigen Toxizitäten ( $\geq$ Grad 3) zur Gesamtzahl, einem bewährten Instrument zur klinischen Beurteilung intensivierterer Therapiemaßnahmen, hatte Kohorte A mit 7,4 \% sogar ein schlechteres Verhältnis als Kohorte B mit 4,8\%. Allerdings sind die relativ geringen OX-assoziierten Nebenwirkungen eher als Folge frühzeitiger Dosismodifikationen als ein Ergebnis besserer Verträglichkeit zu werten. In Bezug auf das 
Tumoransprechen und die Überlebensparameter ist eine um OX erweiterte MMT der MMT mit alleiniger 5-FU-CTx vorzuziehen. Während der perioperativen MMT traten unter FOLFOX-CTx in der Kohorte B 672 Nebenwirkungen auf ( $\Sigma$ Tabellen 17 und 22). Für die Kohorten $A$ ( $\Sigma$ Tabellen 16 und 21) und $C$ ( $\Sigma$ Tabellen 18 und 19) wurden 440 und 441 Ereignisse gezählt. Interessanterweise lagen die behandlungsbedürttigen Nebenwirkungen $\geq$ Grad 3 in den Kohorten $A(n=21)$ und Kohorte $C(n=13)$ deutlich unter den Angaben zu Kohorte $B(n=42)$.

\begin{tabular}{|c|c|c|c|c|c|c|c|c|c|}
\hline \multirow{2}{*}{$\begin{array}{l}\mathrm{n}=52 \text { Patienten (as-treated) } \\
\text { Nebenwirkungen } \\
\text { in CTC-Grade } \geq 1\end{array}$} & \multicolumn{2}{|c|}{ Gesamt } & \multicolumn{7}{|c|}{$\begin{array}{c}\text { Kohorte A } \\
\text { Toxizitätsgrade (NCI-CTCAE Version 4.03) }\end{array}$} \\
\hline & Ereignisse* & $\%$ & 1 & 2 & 3 & 4 & 5 & $\geq 2$ & $\geq 3$ \\
\hline \multicolumn{10}{|l|}{ Unter postop. CTx } \\
\hline Geschmacksinn/-irritation & - & - & - & - & - & - & - & - & - \\
\hline Schmerz & 7 & 3,2 & 2 & 4 & 1 & - & - & 5 & 1 \\
\hline Fatigue & 2 & 0,9 & 2 & - & - & - & - & - & - \\
\hline Hämoglobin & 43 & 19,9 & 33 & 10 & - & - & - & 10 & - \\
\hline Leukozyten & 20 & 9,3 & 11 & 7 & - & 2 & - & 9 & 2 \\
\hline Thrombozyten & 7 & 3,2 & 6 & - & - & 1 & - & 1 & 1 \\
\hline konstitutionelle Symptome ** & 10 & 4,6 & 7 & 3 & - & - & - & 3 & - \\
\hline Kreatinin & 15 & 6,9 & 13 & 2 & - & - & - & 2 & - \\
\hline Haut & 4 & 1,9 & 3 & 1 & - & - & - & 1 & - \\
\hline Stomatitis/Mukositis & 6 & 2,8 & 1 & 5 & - & - & - & 5 & - \\
\hline Zystitis & - & - & - & - & - & - & - & - & - \\
\hline Proktitis & 10 & 4,6 & 3 & 7 & - & - & - & 7 & - \\
\hline intestinale NWK & 18 & 8,3 & 10 & 6 & 2 & - & - & 8 & 2 \\
\hline gastrale NWK & 26 & 12,0 & 20 & 3 & 3 & - & - & 6 & 3 \\
\hline renale NWK & 6 & 2,8 & 2 & 4 & - & - & - & 4 & - \\
\hline neurologische NWK & 4 & 1,9 & 3 & 1 & - & - & - & 1 & - \\
\hline kardiale NWK & 4 & 1,9 & 1 & 2 & - & 1 & - & 3 & 1 \\
\hline Fieber/Infektion & 6 & 2,8 & 2 & 2 & 2 & - & - & 4 & 2 \\
\hline Alopezie & 1 & 0,5 & 1 & - & - & - & - & - & - \\
\hline Allergische Reaktion & 2 & 0,9 & - & 2 & - & - & - & 2 & - \\
\hline $\mathrm{AZ}$ & 1 & 0,5 & - & 1 & - & - & - & 1 & - \\
\hline Dyspnoe & 5 & 2,3 & 4 & 1 & - & - & - & 1 & - \\
\hline IL-6-Syndrom & - & - & - & - & - & - & - & - & - \\
\hline Neutrophile & 1 & 0,5 & - & 1 & - & - & - & 1 & - \\
\hline Thrombose (TVT) & 4 & 1,9 & 1 & 1 & 2 & - & - & 3 & 2 \\
\hline Hepar/Transaminasen, Bilirubin & 7 & 3,2 & 6 & 1 & - & - & - & 1 & - \\
\hline Hand-Fuß-Syndrom & - & - & - & - & - & - & - & - & - \\
\hline Sonstige & 7 & 3,2 & 1 & 4 & 1 & 1 & - & 6 & 2 \\
\hline Summe & 216 & 100,0 & 132 & 68 & 11 & 5 & - & 84 & 16 \\
\hline
\end{tabular}

Tabelle 21: Akute Nebenwirkungen unter adCTx in Kohorte $A$

Die Therapie der Kohorte A ( $n=52$; As-treated-Prinzip) erfolgte nach Komplettierung der präoperativen MMT; *Ereignisse: jedes Ereignis einer akuten hämatologischen und/oder Organ-Toxizität wurde mit dem höchsten CTC-Grad dokumentiert, die während und innerhalb von drei Wochen nach der adCTx aufgetreten waren; **Ernährungszustand und Kognition wurden unter „konstitutionelle Symptome“ zusammengefasst. Farblich hervorgehoben werden die Toxizitäten $\geq$ CTC-Grad 3 mit den häufigsten Symptomkomplexen und der möglichen Konsequenz einer CTXDosisreduktion (z. B. bei Leukozyten $\geq$ CTC-Grad 2). 
Mit den Ereignissen Leukopenie (CTC-Grad 2) und PNP (CTC-Grad 2) erhöhte sich der Anteil an Nebenwirkungen in Kohorte B ( $n=67, \geq \operatorname{Grad} 2)$. Die ab Grad 2 als schwerwiegend empfundene PNP führte zur Dosisreduktion oder CTx-Intervallverlängerung (Tabelle A2: Pat. H19, H45; Tabelle A3: Pat. H118, H149).

\begin{tabular}{|c|c|c|c|c|c|c|c|c|c|}
\hline \multirow{2}{*}{$\begin{array}{l}\mathrm{n}=66 \text { Patienten (as-treated) } \\
\text { Nebenwirkungen } \\
\text { in CTC-Grade } \geq 1\end{array}$} & \multicolumn{2}{|c|}{ Gesamt } & \multicolumn{7}{|c|}{$\begin{array}{c}\text { Kohorte B } \\
\text { Toxizitätsgrade (NCI-CTCAE Version 4.03) }\end{array}$} \\
\hline & Ereignisse* & $\%$ & 1 & 2 & 3 & 4 & 5 & $\geq 2$ & $\geq 3$ \\
\hline \multicolumn{10}{|l|}{ Unter postop. CTx } \\
\hline Geschmacksinn/-irritation & 3 & 0,7 & 1 & 2 & - & - & - & 2 & - \\
\hline Schmerz & 10 & 2,4 & 6 & 4 & - & - & - & 4 & - \\
\hline Fatigue & 5 & 1,2 & 4 & 1 & - & - & - & 1 & - \\
\hline Hämoglobin & 55 & 13,1 & 42 & 12 & 1 & - & - & 13 & 1 \\
\hline Leukozyten & 31 & 7,4 & 21 & 10 & - & - & - & 10 & - \\
\hline Thrombozyten & 7 & 1,7 & 7 & - & - & - & - & - & - \\
\hline konstitutionelle Symptome ** & 20 & 4,8 & 15 & 4 & 1 & - & - & 5 & 1 \\
\hline Kreatinin & 20 & 4,8 & 15 & 4 & - & 1 & - & 5 & 1 \\
\hline Haut & 6 & 1,4 & 4 & 2 & - & - & - & 2 & - \\
\hline Stomatitis/Mukositis & 9 & 2,1 & 6 & 3 & - & - & - & 3 & - \\
\hline Zystitis & 2 & 0,5 & 1 & 1 & - & - & - & 1 & - \\
\hline Proktitis & 10 & 2,4 & 4 & 6 & - & - & - & 6 & - \\
\hline intestinale NWK & 28 & 6,7 & 8 & 15 & 5 & - & - & 20 & 5 \\
\hline gastrale NWK & 74 & 17,7 & 54 & 19 & 1 & - & - & 20 & 1 \\
\hline renale NWK & 9 & 2,1 & 5 & 4 & - & - & - & 4 & - \\
\hline neurologische NWK & 46 & 11,0 & 36 & 8 & 2 & - & - & 10 & 2 \\
\hline kardiale $\mathrm{NWK}^{\star \star *}$ & 3 & 0,7 & 1 & 1 & - & - & 1 & 2 & 1 \\
\hline Fieber/Infektion & 22 & 5,3 & 12 & 5 & 5 & - & - & 10 & 5 \\
\hline Alopezie & 3 & 0,7 & 2 & 1 & - & - & - & 1 & - \\
\hline Allergische Reaktion & 6 & 1,4 & 3 & 3 & - & - & - & 3 & - \\
\hline $\mathrm{AZ}$ & 1 & 0,2 & - & 1 & - & - & - & 1 & - \\
\hline Dyspnoe & 4 & 1,0 & 2 & 1 & 1 & - & - & 2 & 1 \\
\hline IL-6-Syndrom & 3 & 0,7 & 1 & 2 & - & - & - & 2 & - \\
\hline Neutrophile & - & - & - & - & - & - & - & - & - \\
\hline Thrombose (TVT) & 3 & 0,7 & - & 2 & 1 & - & - & 3 & 1 \\
\hline Hepar/Transaminasen, Bilirubin & 31 & 7,4 & 27 & 4 & - & - & - & 4 & - \\
\hline Hand-Fuß-Syndrom & - & - & - & - & - & - & - & - & - \\
\hline Sonstige & 8 & 1,9 & 4 & 3 & 1 & - & - & 4 & 1 \\
\hline Summe & 419 & 100,0 & 281 & 118 & 18 & 1 & 1 & 138 & 20 \\
\hline
\end{tabular}

Tabelle 22: Akute Nebenwirkungen unter adCTx in Kohorte B

Die Therapie der Kohorte B ( $n=66$; As-treated-Prinzip) erfolgte nach der präoperativen MMT; *Ereignisse: jedes Ereignis einer akuten hämatologischen und/oder OrganToxizität wurde mit dem höchsten CTC-Grad dokumentiert, die während und drei Wochen nach der adCTx aufgetreten waren; ${ }^{*}$ Ernährungszustand und Kognition wurden unter "konstitutionelle Symptome“ zusammengefasst. ${ }^{* * *}$ Der Patient mit dem CTCGrad 5 (Sick-Sinus-Syndrom) verstarb 14 Tage nach dem zweiten Zyklus mFOLFOX. Farblich hervorgehoben sind die Toxizitäten $\geq$ CTC-Grad 3 mit den häufigsten Symptomkomplexen und der möglichen Konsequenz einer CTx-Dosisreduktion (z. B. bei Leukozyten $\geq$ CTC-Grad 2). 


\subsection{Therapie-Compliance}

In Tabelle 23 werden die therapieassoziierten Nebenwirkungen nach CTC-Kriterien, die applizierten CTx-Zyklen mit den durchgeführten Dosisreduktionen und die Compliance der Patienten dargestellt. Der Anteil der Grad 3 und Grad 4 Toxizitäten beträgt 4,8 \% (Kohorte A: 1,5\%, Kohorte B: 2,5\% und Kohorte C: 0,8\%). Auffällig sind die hohen Anteile an gastrointestinalen und neurologischen/sensorischen Nebenwirkungen in der Kohorte B (Tabelle 22 und 23), die vor allem während der adCTx aufgetreten waren.

Bis zur TME-OP konnten alle Patienten nach den geplanten Therapieschemata (intention-to-treat) behandelt werden. Bei $98 \%$ aller Patienten erfolgte keine Reduktion der RT-Dosis und bei $95 \%$ keine Dosisreduktion der begleitenden präoperativen CTx. Die nachfolgende mFOLFOX-CTx in Kohorte $\mathrm{C}$ konnte $\mathrm{zu}>86 \%$ in der geplanten Dosis appliziert werden. Zusammengefasst weisen die drei Patienten-Kohorten eine hohe Therapie-Compliance auf. In der postoperativen Behandlungssituation wird dieses hohe Niveau ebenfalls für die ersten beiden Zyklen der adCTx erreicht. Bei den nachfolgenden Zyklen sinkt die Compliance auf lediglich $88,2 \%$ (im dritten Zyklus) und 82,7 \% (im vierten Zyklus). 


\begin{tabular}{|c|c|c|c|c|c|c|c|c|}
\hline & \multicolumn{2}{|c|}{ Gesamt } & \multicolumn{2}{|c|}{$\begin{array}{c}\text { Kohorte A } \\
5 \text {-FU }\end{array}$} & \multicolumn{2}{|c|}{$\begin{array}{l}\text { Kohorte B } \\
5-F U+O X\end{array}$} & \multicolumn{2}{|c|}{$\begin{array}{c}\text { Kohorte C } \\
5-\mathrm{FU}+\mathrm{OX}+\mathrm{mFOLFOX}\end{array}$} \\
\hline & $\mathrm{n}$ & $\%$ & $\mathrm{n}$ & $\%$ & $\mathrm{n}$ & $\%$ & $\mathrm{n}$ & $\%$ \\
\hline \multicolumn{9}{|c|}{ Toxizitäts (Tox.)-grade (NCl-CTCAE Version 4.03) ${ }^{\star}$} \\
\hline$\Sigma$ aller Ereignisse & 1.553 & 100,0 & 482 & 31,0 & 630 & 40,6 & 441 & 28,4 \\
\hline$\Sigma$ Grad 3 / Grad 4 & 75 & 100,0 & 24 & 32,0 & 38 & 50,7 & 13 & 17,3 \\
\hline$\Sigma$ Grad 3 / Grad 4 hämatologisch & 9 & 100,0 & 6 & 66,7 & 2 & 22,2 & 1 & 11,1 \\
\hline$\Sigma$ Grad 3 / Grad 4 gastrointestinal & 30 & 100,0 & 5 & 16,7 & 18 & 60,0 & 7 & 23,3 \\
\hline$\Sigma$ Grade 2 / 3 / 4 Sensorik ${ }^{* *}$ & 35 & 100,0 & 7 & 20,0 & 20 & 57,1 & 8 & 22,9 \\
\hline davon Grad 3 / 4 & 4 & 100,0 & 1 & 25,0 & 3 & 75,0 & - & - \\
\hline$\Sigma$ Grad 3 / Grad 4 laborchemisch & 1 & 100,0 & - & - & 1 & 100,0 & - & - \\
\hline$\Sigma$ Grad 3 / Grad 4 kardial & 1 & 100,0 & 1 & 100,0 & - & - & - & - \\
\hline$\Sigma$ Grad 3 / Grad 4 urologisch & 3 & 100,0 & - & - & 2 & 66,7 & 1 & 33,3 \\
\hline$\Sigma$ weitere Tox. Grad 3 / Grad 4 & 27 & 100,0 & 8 & 29,6 & 15 & 55,6 & 4 & 14,8 \\
\hline \multicolumn{9}{|c|}{ Therapie-Compliance ${ }^{\star \star \star}$ (präoperativer RT) } \\
\hline \multicolumn{9}{|c|}{ Radiatio (50,4 Gy) } \\
\hline komplett appliziert & 173 & 97,7 & 64 & 100,0 & 59 & 93,7 & 50 & 100,0 \\
\hline mit Dosisreduktion & 4 & 2,3 & - & - & 4 & 6,3 & - & - \\
\hline \multicolumn{9}{|c|}{ Therapie-Compliance ${ }^{\star \star \star}($ präoperativer CRT) } \\
\hline \multicolumn{9}{|c|}{ Anzahl Patienten mit vollständigen CTx Zyklen / Applikationen } \\
\hline $1(5-F U) / 1+2$ (FOLFOX) & 172 & 97,2 & 63 & 98,4 & 60 & 95,2 & 49 & 98,0 \\
\hline $2(5-F U) / 3+4$ (FOLFOX) & 168 & 94,9 & 63 & 98,4 & 56 & 88,9 & 49 & 98,0 \\
\hline \multicolumn{9}{|c|}{ 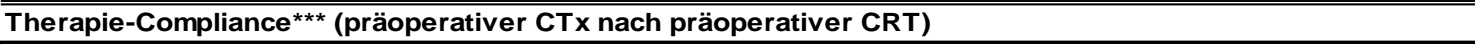 } \\
\hline \multicolumn{9}{|c|}{ Anzahl Patienten mit vollständigen präoperativer CTx Applikationen } \\
\hline 1 (mFOLFOX) & 45 & 90,0 & - & - & - & - & 45 & 90,0 \\
\hline 2 (mFOLFOX) & 43 & 86,0 & - & - & - & - & 43 & 86,0 \\
\hline 3 (mFOLFOX) & 46 & 92,0 & - & - & - & - & 46 & 92,0 \\
\hline \multicolumn{9}{|c|}{ Therapie-Compliance ${ }^{\star \star \star \star}(\operatorname{adCT} x)$} \\
\hline \multicolumn{9}{|c|}{ Anzahl Patienten mit vollständigen adCTx Zyklen / Applikationen } \\
\hline $1(5-\mathrm{FU}) / 1+2$ (FOLFOX) & 118 & 100,0 & 52 & 100,0 & 66 & 100,0 & - & - \\
\hline 2 (5-FU) / $3+4$ (FOLFOX) & 116 & 98,3 & 51 & 98,1 & 65 & 98,5 & - & - \\
\hline $3(5-\mathrm{FU}) / 5+6$ (FOLFOX) & 112 & 94,9 & 50 & 96,2 & 62 & 93,9 & - & - \\
\hline 4 (5-FU) / $7+8$ (FOLFOX) & 105 & 89,0 & 49 & 94,2 & 56 & 84,8 & - & - \\
\hline \multicolumn{9}{|c|}{ Zyklen / Applikationen CTx und Radiatio } \\
\hline$\Sigma$ & 1.153 & 100,0 & 400 & 100,0 & 453 & 100,0 & 300 & 100,0 \\
\hline komplett applizierte MMT & 1.012 & 87,8 & 376 & 94,0 & 354 & 78,1 & 282 & 94,0 \\
\hline mit Dosisreduktion & 141 & 12,2 & 24 & 6,0 & 99 & 21,9 & 18 & 6,0 \\
\hline$\Sigma$ präoperativ & 681 & 100,0 & 192 & 100,0 & 189 & 100,0 & 300 & 100,0 \\
\hline präoperativ mit geplanter Dosis & 648 & 95,2 & 190 & 99,0 & 176 & 93,1 & 282 & 94,0 \\
\hline präoperativ mit Dosisreduktion & 33 & 4,8 & 2 & 1,0 & 13 & 6,9 & 18 & 6,0 \\
\hline$\Sigma$ postoperativ & 472 & 20,5 & 208 & 26,0 & 264 & 29,1 & - & - \\
\hline postoperativ mit geplanter Dosis & 365 & 77,3 & 186 & 89,4 & 179 & 67,8 & - & - \\
\hline postoperativ mit Dosisreduktion & 107 & 22,7 & 22 & 10,6 & 85 & 32,2 & - & - \\
\hline
\end{tabular}

Tabelle 23: Therapie-assoziierte Nebenwirkungen und Therapie-Compliance

MMT: multimodale Therapie; *Toxizität: NCI-CTCAE Grade 1-4; bei einem Patienten trat eine Toxizität Grad 5 (H161; Kohorte B; kardial bedingter Exitus; Tabelle A4) auf; ein weiterer Patient (H64; Kohorte A, Tabelle A1) war 10 Tage nach Relaparotomie aufgrund einer Lungenembolie gestorben; ${ }^{* *}$ sensorische Toxizität der Grade $2-4 ;{ }^{* * *}$ die Prozentangabe bezieht sich auf die Patienten Kohorten (Kohorte A: $n=64$, Kohorte B: $n=63$, Kohorte C: $n=50)$ oder Gesamtzahl $(n=177)$; ${ }^{* * *}$ bei 14 Patienten wurde die geplante adCTx geändert; die Prozentangaben beziehen sich nach dem As-treated-Prinzip auf alle mit postoperativer CTx behandelten Patienten (Kohorte A: $n=52$, Kohorte B: $n=66$ ). 


\subsection{Nachsorge - Lokalrezidive und Fernmetastasen}

Nach Abschluss der perioperativen Multimodalbehandlung traten 166 (93,8 \%) der 177 Patienten in die geplante Nachsorge-Phase ein. Diese Patienten standen im Fokus von Überlebenszeitanalysen (Abbildung 13). Während der Nachbeobachtung (Median: 73 Monate; Mittelwert: 77,3 Monate; Min.-Max.: 03-149 Monate) traten bei 52 (31,3 \%) von 166 Patienten Fernmetastasen auf (Tabelle 24).

\begin{tabular}{|c|c|c|c|c|c|c|c|c|}
\hline & \multicolumn{2}{|c|}{ Gesamt } & \multicolumn{2}{|c|}{$\begin{array}{c}\text { Kohorte A } \\
\text { 5-FU }\end{array}$} & \multicolumn{2}{|c|}{$\begin{array}{l}\text { Kohorte B } \\
5-F U+O X\end{array}$} & \multicolumn{2}{|c|}{$\begin{array}{c}\text { Kohorte C } \\
5-F U+O X+\text { mFOLFOX }\end{array}$} \\
\hline & $n$ & $\%$ & $n$ & $\%$ & $\mathrm{n}$ & $\%$ & $n$ & $\%$ \\
\hline $\begin{array}{l}\Sigma \text { Fernmetastasen } \\
(\text { FM) }\end{array}$ & 52 & 100,0 & 16 & 100,0 & 26 & 100,0 & 10 & 100,0 \\
\hline \multicolumn{9}{|l|}{ Primärlokalisation FM } \\
\hline hepar & 22 & 42,3 & 3 & 18,8 & 16 & 61,5 & 3 & 30,0 \\
\hline pulmonal & 17 & 32,7 & 8 & 50,0 & 6 & 23,1 & 3 & 30,0 \\
\hline peritoneal & 9 & 17,3 & 4 & 25,0 & 2 & 7,7 & 3 & 30,0 \\
\hline ossär & 3 & 5,8 & 1 & 6,3 & 1 & 3,8 & 1 & 10,0 \\
\hline inguinal & 1 & 1,9 & - & - & 1 & 3,8 & - & - \\
\hline \multicolumn{9}{|c|}{ Anzahl der von FM-betroffenen Organe } \\
\hline 1 Organ & 41 & 78,8 & 14 & 87,5 & 20 & 76,9 & 7 & 70,0 \\
\hline 2 Organe & 8 & 15,4 & 2 & 12,5 & 3 & 11,5 & 3 & 30,0 \\
\hline 3 Organe & 1 & 1,9 & - & - & 1 & 3,8 & - & - \\
\hline$>3$ Organe & 2 & 3,8 & - & - & 2 & 7,7 & - & - \\
\hline \multicolumn{9}{|c|}{ Auftreten von FM und Lokalrezidiven (LR) } \\
\hline Anzahl Patienten & 166 & 100,0 & 52 & 100,0 & 66 & 100,0 & 48 & 100,0 \\
\hline davon nur FM & 43 & 25,9 & 12 & 23,1 & 21 & 31,8 & 10 & 20,8 \\
\hline davon nur LR & & - & - & - & & - & - & - \\
\hline davon FM und LR & 9 & 5,4 & 4 & 7,7 & 5 & 7,6 & - & - \\
\hline davon keine & 114 & 68,7 & 36 & 69,2 & 40 & 60,6 & 38 & 79,2 \\
\hline
\end{tabular}

Tabelle 24: Auftreten von Lokalrezidiven (LR) und Fernmetastasen (FM)

Die Angabe der fernmetastatischen Rezidive während der Nachsorge (nach Therapieabschluss) von 166 Patienten erfolgt nach der primären Lokalisation und der Anzahl an betroffenen Organsystemen; FM und LR: bei diesen Patienten traten neben den FM im Verlauf auch lokoregionäre Rezidive auf.

Bei den Patienten der Kohorte $A(n=52)$ wurden in 16 Fällen $(31 \%)$, in der Kohorte $B(n=66)$ in 26 Fällen (39\%) und in der Kohorte $C(n=48)$ in 10 Fällen (21\%) Fernmetastasen nachgewiesen. Im kürzeren Nachbeobachtungszeitraum der Kohorte $C$ traten bisher weniger FM auf (Tabelle 24). Lokoregionäre Rezidive (LR) wurden insgesamt bei neun Patienten (5,0 \%) zeitgleich mit den FM oder se- 
kundär diagnostiziert. Ein alleiniges Lokalrezidiv wurde nicht entdeckt.

Die FM waren in 22 Fällen in der Leber (Kohorte A: $n=3$, Kohorte $B: n=16$, Kohorte $C: n=3$ ), in 17 Fällen in der Lunge (Kohorte $A: n=8$, Kohorte $B: n=6$, Kohorte $C: n=3$ ) und bei einem Patienten zeitgleich intrahepatisch und pulmonal festgestellt worden. Bei einem Patienten (Kohorte B) lag eine inguinale FM vor. In drei Fällen konnten ossäre (Kohorte A, B und C mit jeweils einem Patienten) und bei neun Studienteilnehmern intraperitoneale Metastasen (Kohorte $A: n=4$, Kohorte $B: n=2$, Kohorte $C: n=3$ ) diagnostiziert werden. Insgesamt wurden bei 41 Patienten (Kohorte $A: n=14$, Kohorte $B: n=20$, Kohorte $C: n=7$ ) FM in einem Organsystem nachgewiesen. Polytope FM wurden bei acht Patienten (Kohorte $A: n=2$, Kohorte $B: n=3$, Kohorte $C: n=3$ ) in zwei Organsystemen, bei einem Patienten (Kohorte B) in drei Organsystemen und bei 2 Patienten (Kohorte B: $n=2)$ in > 3 Organsystemen entdeckt. Bis zum letzten Tag der Datenerhebung (31.12.2018) waren 22,0 \% $(n=39)$ der 177 Patienten aufgrund ihrer Malignomerkrankung gestorben.

Weitere 31 (17,5\%) Patienten starben während der Nachsorgephase nicht Rektumkarzinom-bedingt infolge einer schweren Herzinsuffizienz $(n=5)$, einer pulmonalen Infektion $(n=2)$, einer Lungenembolie $(n=4)$ oder fortschreitender Sekundärmalignome $(n=5)$ sowie anderer Ursache (Sepsis: $n=4$; Apoplex: $n=2$; Suizid: $\mathrm{n}=1$ ).

Bei insgesamt neun (5,4 \%) von 166 Patienten, die sich der Tumornachsorge unterzogen hatten (Patient H161 verstarb noch während der adCTx-Phase, Tabelle A4), wurden sekundäre Malignome anderer Entitäten festgestellt. Diese Malignomerkrankungen wurden als chronische lymphatische Leukämie (CLL, $n=1 ; H 41$ ), Harnblasenkarzinome $(n=2 ; H 24, H 152)$, Vulvakarzinom $(n=1 ; H 61)$ und Mammakarzinom ( $n=1$; H87) sowie HCC ( $n=1$; H9) diagnostiziert. Bei drei Patienten (H41, H103, H116) traten sekundäre Darmkrebserkrankungen auf. Der Patient H41 wurde aufgrund eines sekundären Karzinoms im Colon ascendens (Stadium pUICC-II) mit einer Hemikolektomie (rechts) ohne postoperativer adCTx kurativ behandelt (bisheriges OS/CSS: 121 Monate, DFS/RFS: 10 Monate). Der Patient $\mathrm{H} 103$ entwickelte bei polytoper pulmonaler Metastasierung unter Therapie mit FOLFIRI + Cetuximab sowie anschließender FOLFOX + Cetuximab Behandlung 
ein Zweitkarzinom im Colon ascendens. Nach rechtseitiger Hemikolektomie starb der Patient am Progress der pulmonalen Metastasierung (CSS: 42 Monate, RFS: 12 Monate). Der dritte Patient, Fall H116 mit einem sekundären Sigmakarzinom (pUICC-III), erhielt postoperativ eine adCTx mit sechs Zyklen (12 Applikationen) FOLFIRI in kurativer Intention. Dieser Patient ist weiterhin tumorfrei und das OS/CSS beträgt bislang 71 Monate (DFS: 55 Monate, RFS: 56 Monate).

\subsection{Nachsorge - Überlebenszeiten und Lebensalter}

Das mittlere DFS nach der TME betrug für 167 Patienten 91,47 Monate, das RFS 92,61 Monate, das CSS 121,67 Monate und das OS 105,89 Monate (Tabelle 25).

\begin{tabular}{|c|c|c|c|c|}
\hline & DFS & RFS & CSS & OS \\
\hline \multicolumn{5}{|c|}{ Kohorte A, B und C in Summe nach Altersgruppen } \\
\hline \multicolumn{5}{|c|}{ bis 54 Jahre } \\
\hline $\mathrm{rMW}^{*}$ & 83,76 & 88,40 & 112,32 & 108,44 \\
\hline $95 \%-\mathrm{Cl}$ & $64,92-102,60$ & $69,11-107,69$ & $95,87-128,77$ & $91,97-124,92$ \\
\hline \multicolumn{5}{|c|}{$>54$ bis 63 Jahre } \\
\hline $\mathrm{rMW}^{*}$ & 95,51 & 95,51 & 122,00 & 105,54 \\
\hline $95 \%-\mathrm{Cl}$ & $78,32-112,70$ & $78,32-112,70$ & $108,09-135,92$ & $90,16-120,91$ \\
\hline \multicolumn{5}{|c|}{$>63$ bis 69 Jahre } \\
\hline $\mathrm{rMW}^{*}$ & 93,04 & 93,04 & 115,61 & 99,8 \\
\hline $95 \%-\mathrm{Cl}$ & $74,66-111,41$ & $74,66-111,41$ & $100,05-131,17$ & $82,61-117,00$ \\
\hline \multicolumn{5}{|l|}{$>69$ Jahre } \\
\hline $\mathrm{rMW}^{*}$ & 87,20 & 87,20 & 124,52 & 102,16 \\
\hline $95 \%-\mathrm{Cl}$ & $69,28-105,12$ & $69,28-105,12$ & $111,46-137,59$ & $87,06-117,25$ \\
\hline \multicolumn{5}{|l|}{ gesamt } \\
\hline $\mathrm{rMW}^{*}$ & 91,47 & 92,61 & 121,67 & 105,86 \\
\hline $95 \%-\mathrm{Cl}$ & $82,08-100,86$ & $83,19-102,04$ & $113,83-129,50$ & $97,50-114,22$ \\
\hline logrank** & $p=0,695$ & $p=0,839$ & $p=0,536$ & $p=0,951$ \\
\hline
\end{tabular}

Tabelle 25: Überlebenszeit nach Altersgruppen (Quartile)

$\mathrm{rMW}^{*}$ : beschränkte erwartete Überlebenszeit (restricted mean of survival time) als Mittelwert für das jeweilige Überleben, diese Schätzung ist auf die längste Überlebenszeit begrenzt, wenn sie zensiert wurde; 95\%-Cl: 95\%-Konfidenzintervall; ${ }^{* *} \mathrm{p}$-Werte nach logrankTest 
Beim Vergleich der Altersgruppen zeigt sich kein signifikanter Unterschied in den Überlebenszeiten (Tabelle 25 und 26). Dabei wurden in der Gruppe der $<49$ Jährigen vier Patienten miterfasst, die jünger als 40 Jahre waren. Auch in der Altersgruppe der > 70-Jährigen wurden vier Patienten berücksichtigt, die zum Zeitpunkt der Diagnosestellung älter als 79 Jahre waren (Tabelle 26).

\begin{tabular}{|c|c|c|c|c|}
\hline & DFS & RFS & CSS & os \\
\hline \multicolumn{5}{|c|}{ Kohorte A, B und C in Summe nach Altersgruppen } \\
\hline \multicolumn{5}{|c|}{\begin{tabular}{|l|l|l} 
bis 49 Jahre $^{*}$ & & \\
\end{tabular}} \\
\hline $\mathrm{rMW}^{* *}$ & 92,35 & 92,35 & 108,47 & 105,88 \\
\hline $95 \%-\mathrm{Cl}$ & $64,52-120,18$ & $64,52-120,18$ & $84,01-132,92$ & $81,94-129,82$ \\
\hline \multicolumn{5}{|l|}{50 bis 59 Jahre } \\
\hline $\mathrm{rMW}^{* *}$ & 88,02 & 92,05 & 120,30 & 107,73 \\
\hline $95 \%-\mathrm{Cl}$ & $70,992-105,039$ & $74,51-109,58$ & $105,75-134,84$ & $92,26-123,19$ \\
\hline \multicolumn{5}{|l|}{60 bis 69 Jahre } \\
\hline $\mathrm{rMW}^{* *}$ & 91,16 & 91,16 & 114,95 & 100,44 \\
\hline $95 \%-\mathrm{Cl}$ & $76,55-105,76$ & $76,55-105,76$ & $102,86-127,04$ & $87,14-113,73$ \\
\hline \multicolumn{5}{|l|}{ ab 70 Jahre* } \\
\hline$r \mathrm{rMW}^{\star *}$ & 87,20 & 87,20 & 124,520 & 102,16 \\
\hline $95 \%-\mathrm{Cl}$ & $69,28-105,12$ & $69,28-105,12$ & $111,46-137,60$ & $87,06-117,25$ \\
\hline \multicolumn{5}{|l|}{ gesamt } \\
\hline $\mathrm{rMW}^{\star \star *}$ & 91,47 & 92,61 & 121,67 & 105,86 \\
\hline $95 \%-\mathrm{Cl}$ & $82,08-100,86$ & $83,19-102,04$ & $113,83-129,50$ & $97,50-114,22$ \\
\hline $\operatorname{logran}^{\star * *}$ & $p=0,897$ & $p=0,926$ & $p=0,593$ & $p=0,945$ \\
\hline
\end{tabular}

Tabelle 26: Überlebenszeit nach Clusterung des Lebensalters in Dekaden

* beim Clustern bis 49 Jahre und ab 70 Jahre wurden jeweils vier Patienten erfasst, deren Lebensalter außerhalb der Dekade lag; ${ }^{* *} r M W$ : beschränkte erwartete Überlebenszeit (restricted mean of survival time) als Mittelwert für das jeweilige Überleben, diese Schätzung ist auf die längste Überlebenszeit begrenzt, wenn sie zensiert wurde; 95\%-Cl: 95\%Konfidenzintervall; ${ }^{* * *} p$-Werte nach logrank-Test

\section{9 Überleben nach perioperativer Multimodaltherapie}

In den folgenden Abschnitten werden das DFS, RFS, CSS und OS für jene 167 Patienten angegeben, die nach erfolgter präoperativer MMT bereit waren, die geplante postoperative Weiterbehandlung und Nachsorge anzutreten. Dabei bestand die adCTx für Kohorte A aus vier Zyklen (vier Applikationen, 1-5 d) 5-FU-Monotherapie und für Kohorte B aus vier Zyklen FOLFOX (acht Applikationen, Abbil- 
dung 13). Die Überlebenszeitanalysen erfolgten nach den Intention-to-treat- und As-treated-Verfahrensweisen, wie jeweils in den Legenden der nachfolgenden Darstellungen im Ergebnisteil angegeben. Dabei wurden unter besonderen Fragestellungen auch diejenigen Patienten ausgewertet, die die adCTx gewechselt hatten (neun Patienten von Kohorte A nach B; zwei Patienten von Kohorte B nach A).

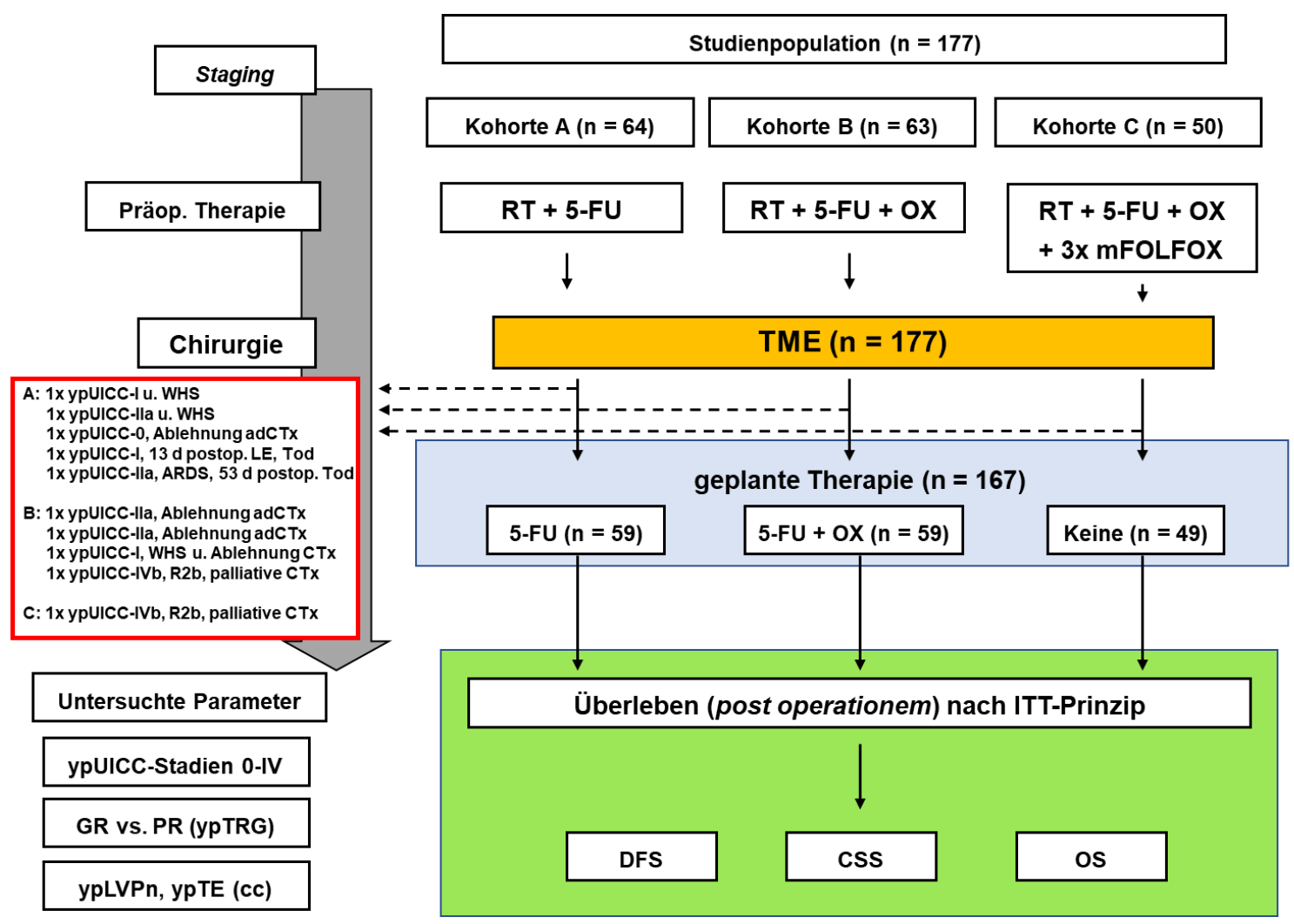

Abbildung 13: Studienpopulation und Auswertungsparameter (I) nach MMT Eigene Darstellung; die Auswertung erfolgte nach präoperativer Multimodaltherapie, TME und Start der postoperativen Therapie (+/- adCTx; $n=167$ Patienten) 


\subsection{Univariate Survivalanalysen zu den ypUICC-Stadien}

\subsection{1 ypUICC-Stadien - krankheitsfreies Überleben (DFS)}

Das krankheitsfreie Überleben (DFS) aller 167 Patienten mit einem initial fortgeschrittenen Rektumkarzinom (Kohorten A-C) betrug in den Stadien ypUICC-0 und ypUICC-I im Median 140 Monate bzw. 106 Monate. Demgegenüber hatten Patienten mit Tumorstadien ypUICC-III und ypUICC-IV ein medianes DFS von 84 Monaten (95\%-Cl: 29,19-138,81 Monate) und 9 Monaten (95\%-Cl: 2,37-15,63 Monate).

\begin{tabular}{|c|c|c|c|c|c|c|c|}
\hline \multirow{3}{*}{$\frac{\text { Stadium }}{\text { ypUICC-0 }}$} & \multirow{3}{*}{$\frac{N}{25}$} & \multirow{3}{*}{$\begin{array}{r}\text { Ereignisse } \\
3\end{array}$} & \multicolumn{4}{|c|}{ Krankheitsfreies Überleben (DFS, in Monaten) } & \multirow[b]{2}{*}{ logrank*** } \\
\hline & & & $r M W^{*}$ & SD & \multicolumn{2}{|c|}{$95 \% \mathrm{Cl}^{* *}$} & \\
\hline & & & 133,78 & 6,54 & 120,96 & 146,59 & \multirow{6}{*}{$p<0,0009$} \\
\hline ypUICC-I & 38 & 17 & 96,74 & 8,82 & 79,46 & 114,02 & \\
\hline ypUICC-II & 45 & 20 & 92,11 & 8,38 & 75,70 & 108,53 & \\
\hline ypUICC-III & 44 & 25 & 79,99 & 9,26 & 61,83 & 98,14 & \\
\hline ypUICC-IV & 15 & 13 & 22,87 & 8,86 & 5,51 & 40,23 & \\
\hline$\Sigma$ & 167 & 78 & 91,47 & 4,79 & 82,08 & 100,86 & \\
\hline
\end{tabular}

Tabelle 27: DFS (rMW) in Abhängigkeit von den ypUICC-Stadien 0-IV $\mathrm{rMW}^{*}$ : beschränkte erwartete Überlebenszeit (restricted mean of disease-free survival) als Mittelwert für das krankheitsfreie Überleben (DFS); diese Schätzung ist auf die längste Überlebenszeit begrenzt, wenn sie zensiert wurde; SD: Standardabweichung; $95 \%-\mathrm{Cl}^{* *}$ : 95\%-Konfidenzintervall; Testung auf Gleichheit der Überlebensverteilung in der KaplanMeier (K-M)-Darstellung für die verschiedenen ypUICC-Stadien: Chi-Quadrat 50,908, 4 Freiheitsgrade; logrank ${ }^{* * *}$ : logrank-Test: $p<0,0009$

Das beschränkte, erwartete mittlere DFS (rMW, Tabelle 27) in den K-MÜberlebenskurven (Abbildung 14) unterscheidet sich in den Tumorstadien signifikant (logrank-Test: $p<0,0009$ ). Es zeigte sich ein exzellentes DFS für Patienten mit einem ypUICC-0 Stadium nach perioperativer MMT. Es waren nur drei Ereignisse bei den 25 Patienten mit CR aufgetreten. Bei den Patienten mit den Stadien ypUICC-I und ypUICC-II sind innerhalb der ersten fünf Jahre der Langzeitbeobachtung passagere Unterschiede im DFS festzustellen (ypUICC-I: DFS (rMW): 96,74 Monate; 95\%-Cl: 79,46-114,02 Monate; ypUICC-II: DFS (rMW): 92,11 Monate; 95\%-Cl: 75,70-108,53 Monate). Allerdings grenzen die Patienten sich deutlich von jenen Fällen mit einem ypUICC-III Stadium ab (DFS (rMW): 79,99 Mona- 
te; 95\%-Cl: 61,83-98,14 Monate). Patienten mit einem ypUICC-IV Stadium hatten ein DFS (rMW) von 22,87 Monaten (95\%-Cl: 5,51-40,23 Monate). Bei einer im Verhältnis zur Patientenzahl erwartungsgemäß hohen Anzahl an Ereignissen (Tabelle 27; $87 \%$ ) haben sie gegenüber den anderen Tumorstadien (12-57\%) ein deutlich schlechteres krankheitsfreies Überleben.

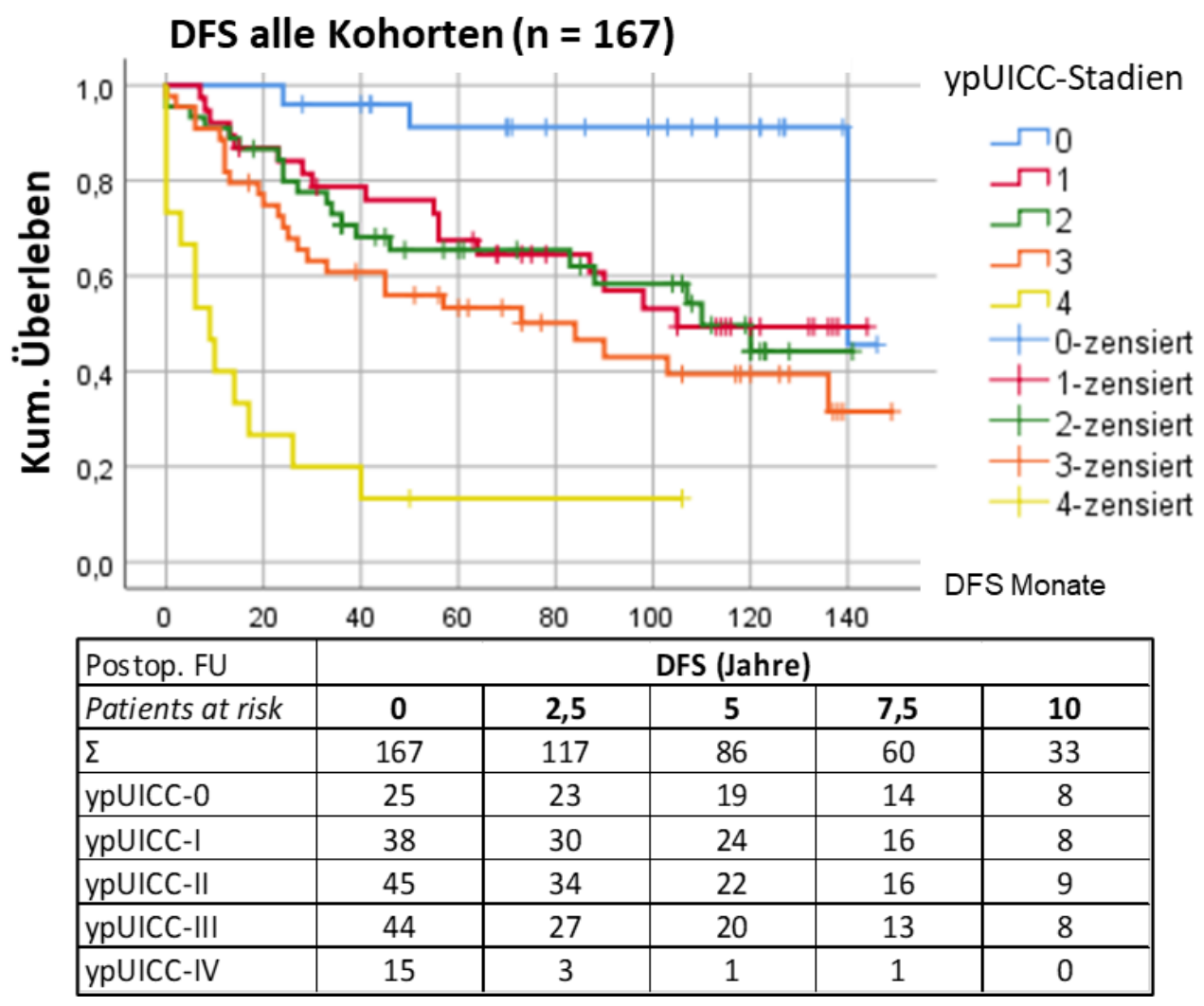

Abbildung 14: DFS in Abhängigkeit von den ypUICC-Stadien 0-IV Postop. FU: postoperatives Follow up

\subsection{2 ypUICC-Tumorstadien - rezidivfreies Überleben (RFS)}

Das mediane rezidivfreie Überleben (RFS) aller 167 Patienten beträgt im Stadium ypUICC-0 140 Monate, im Stadium ypUICC-II 110 Monate, im Stadium ypUICC-III 84 Monate sowie 9 Monate im ypUICC-IV. Für das ypUICC-I Tumorstadium kann kein RFS-Medianwert angegeben werden. Folglich sind in Tabelle 28 die rMW für das RFS, die Anzahl der Ereignisse und das $95 \%-\mathrm{Cl}$ in Abhängigkeit zu den Stadien ypUICC-0 bis ypUICC-IV aufgeführt. Es besteht im Vergleich zu den Angaben des DFS nur ein geringer Unterschied, sodass in den folgenden Abschnitten auf 
die Darstellung des RFS verzichtet wird.

\begin{tabular}{|c|c|c|c|c|c|c|c|}
\hline \multirow{3}{*}{$\frac{\text { Stadium }}{\text { ypUICC-0 }}$} & \multirow{3}{*}{$\frac{N}{25}$} & \multirow{3}{*}{$\frac{\text { Ereignisse }}{3}$} & \multicolumn{4}{|c|}{ Überleben (RFS, in Monaten) } & \multirow[b]{2}{*}{ logrank*** } \\
\hline & & & $\mathrm{rMW}^{*}$ & SD & \multicolumn{2}{|c|}{$95 \% \mathrm{Cl}^{* *}$} & \\
\hline & & & 133,78 & 6,54 & 120,96 & 146,59 & \multirow{6}{*}{$p<0,0009$} \\
\hline ypUICC-I & 38 & 15 & 102,04 & 9,19 & 84,03 & 120,04 & \\
\hline ypUICC-II & 45 & 20 & 92,11 & 8,38 & 75,70 & 108,53 & \\
\hline ypUICC-III & 44 & 25 & 79,96 & 9,26 & 61,83 & 98,14 & \\
\hline ypUICC-IV & 15 & 13 & 22,87 & 8,86 & 5,51 & 40,23 & \\
\hline$\Sigma$ & 167 & 76 & 92,61 & 4,81 & 83,19 & 102,04 & \\
\hline
\end{tabular}

Tabelle 28: RFS (rMW) in Abhängigkeit von den ypUICC-Stadien 0-IV

$\mathrm{rMW}^{*}$ : beschränkte erwartete Überlebenszeit (restricted mean of RFS) als Mittelwert für das RFS, diese Schätzung ist auf die längste Überlebenszeit begrenzt, wenn sie zensiert wurde; SD: Standardabweichung; 95\%-Cl**: 95\%-Konfidenzintervall; Testung auf Gleichheit der Überlebensverteilung in der K-M-Darstellung für die ypUICC-Stadien: Chi-Quadrat 52,0, 4 Freiheitsgrade; logrank ${ }^{* * *}$ : logrank-Test: $p<0,0009$

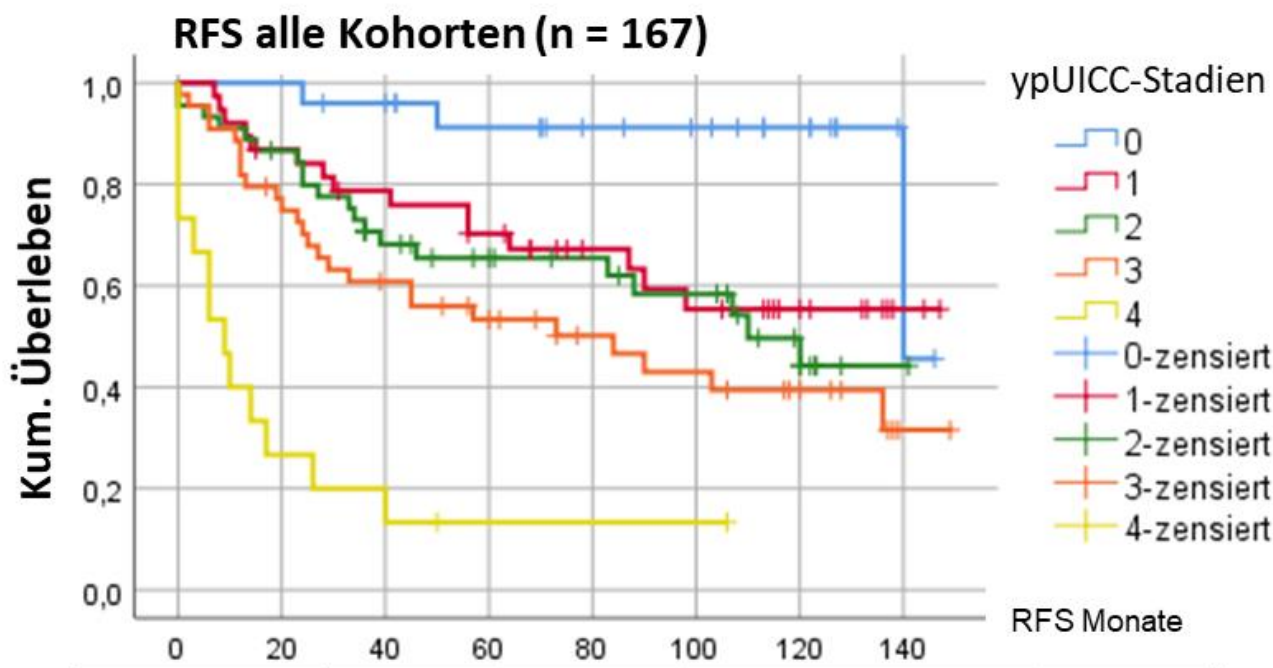

\begin{tabular}{|l|c|c|c|c|c|}
\hline Postop. FU & \multicolumn{5}{|c|}{ RFS (Jah re) } \\
\hline Patients at risk & $\mathbf{0}$ & $\mathbf{2 , 5}$ & $\mathbf{5}$ & $\mathbf{7 , 5}$ & $\mathbf{1 0}$ \\
\hline$\Sigma$ & 167 & 117 & 86 & 60 & 35 \\
\hline ypUICC-0 & 25 & 23 & 19 & 14 & 8 \\
\hline ypUICC-I & 38 & 30 & 24 & 16 & 9 \\
\hline ypUICC-II & 45 & 34 & 22 & 16 & 9 \\
\hline ypUICC-III & 44 & 27 & 20 & 13 & 9 \\
\hline ypUICC-IV & 15 & 3 & 1 & 1 & 0 \\
\hline
\end{tabular}

Abbildung 15: RFS in Abhängigkeit von den ypUICC-Stadien 0-IV Postop. FU: postoperatives Follow up 
Die Analyse des RFS zeigt eine noch deutlichere prognostische Abgrenzbarkeit der Patienten mit $\leq$ ypUICC-II Tumorstadien gegenüber den Stadien ypUICC-III und ypUICC-IV. Neben dem exzellenten Krankheitsverlauf von Patienten mit einer CR haben auch Patienten mit einem ypUICC-I Stadium ein gutes Langzeitansprechen auf die MMT.

\subsection{3 ypUICC-Stadien - krebsspezifisches Überleben (CSS)}

Das mediane krebsspezifische Überleben (CSS) beträgt nach perioperativer MMT bei Patienten mit einem Tumorstadium ypUICC-IV 47,0 Monaten. Wie aus den signifikant unterschiedlich verlaufenden K-M-Überlebenskurven (logrank-Test: $\mathrm{p}<0,0009)$ für das CSS deutlich wird, haben Patienten mit ypUICC-0 bis -II Stadien ein sehr gutes Rektumkarzinom-freies Langzeitüberleben ( $\geq 80 \%$ ) unabhängig von der tatsächlich durchgeführten perioperativen MMT. Der rMW für das CSS von Patienten mit einer CR (ypUICC-0 Stadium) beträgt 141,94 Monate $( \pm 3,94$ Monate; 95\%-Cl: 134,22-149,67 Monate), für Patienten mit einem Stadium ypUICC-I 133,82 Monate ( \pm 6,28 Monate; 95\%-Cl: 121,52-146,12 Monate) und für Patienten mit einem ypUICC-II Stadium 121,89 Monate ( \pm 7,00 Monate; $95 \%-\mathrm{Cl}$ : 108,17-135,62). Demgegenüber sind die rMW des CSS für Patienten mit einem residuellen ypUICC-III Stadium oder gar ypUICC-IV Stadium auf 111,50 Monaten ( \pm 8,48 Monate; 95\%-Cl: 94,89-128,12 Monate) und 56,31 Monate ( \pm 11,28 Monate; $95 \%-\mathrm{Cl}: 34,21-78,42$ Monate, Tabelle 29) reduziert. Unter Berücksichtigung der Studieneingangskriterien scheinen Patienten mit einem Tumorstadium $\leq$ ypUICC-II die eigentlichen "Gewinner" der perioperativen MMT zu sein. Diese These muss allerdings in multiparametrischen Überlebensmodellen überprüft werden (Abschnitt 3.15). Ähnlich wie bei den K-M-Kurven zum RFS kann bei den CSSÜberlebenskurven über zwei prognostisch unterschiedliche Subgruppen im ypUICC-II Stadium diskutiert werden. Zudem ist auch das CSS von Patienten im Stadium ypUICC-IV bemerkenswert. Trotz dieses allgemein infausten Status leben immerhin noch $50 \%$ der Patienten 45 Monate post operationem. Ein Ergebnis, das vor Einführung multimodaler Therapiekonzepte undenkbar war (Sahlmann et al. 2017; Liersch et al. 2007b). 


\begin{tabular}{|c|c|c|c|c|c|c|c|}
\hline \multirow{3}{*}{$\frac{\text { Stadium }}{\text { ypUICC-0 }}$} & \multirow{3}{*}{$\frac{N}{25}$} & \multirow{3}{*}{$\frac{\text { Ereignisse }}{1}$} & \multicolumn{4}{|c|}{ Überleben (CSS, in Monaten) } & \multirow[b]{2}{*}{$\log r a n k^{* * *}$} \\
\hline & & & $\mathrm{rMW}^{*}$ & SD & \multicolumn{2}{|c|}{$95 \%-\mathrm{Cl}^{* *}$} & \\
\hline & & & 141,94 & 3,94 & 134,22 & 149,67 & \multirow{6}{*}{$p<0,0009$} \\
\hline ypUICC-I & 38 & 4 & 133,82 & 6,28 & 121,52 & 146,12 & \\
\hline ypUICC-II & 45 & 8 & 121,89 & 7,00 & 108,17 & 135,62 & \\
\hline ypUICC-III & 44 & 13 & 111,50 & 8,48 & 94,89 & 128,12 & \\
\hline ypUICC-IV & 15 & 10 & 56,31 & 11,28 & 34,21 & 78,42 & \\
\hline $\boldsymbol{\Sigma}$ & 167 & 36 & 121,67 & 4,00 & 113,83 & 129,50 & \\
\hline
\end{tabular}

Tabelle 29: CSS (rMW) in Abhängigkeit von den ypUICC-Stadien 0-IV

$\mathrm{rMW}^{*}$ : beschränkte erwartete Überlebenszeit (restricted mean of CSS) als Mittelwert für das krebsspezifische Überleben (CSS); die Schätzung ist auf die längste Überlebenszeit begrenzt, wenn sie zensiert ist; SD: Standardabweichung; $95 \%-\mathrm{Cl}^{* *}$ : 95\%-Konfidenzintervall; Testung auf Gleichheit der Überlebensverteilung in der Kaplan-Meier-Darstellung für die verschiedenen ypUICC-Stadien: Chi-Quadrat 36,234, 4 Freiheitsgrade; logrank ${ }^{\star * *}$ logrank-Test: $p<0,0009$

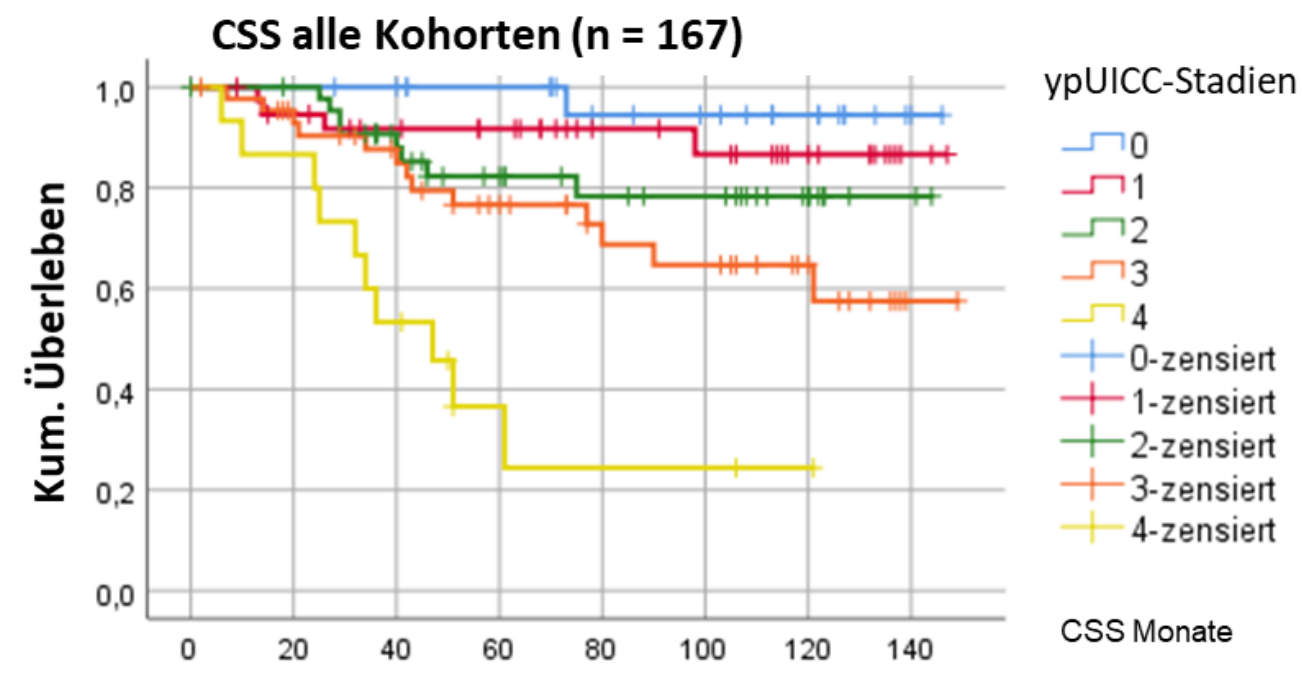

\begin{tabular}{|l|c|c|c|c|c|}
\hline Postop. FU & \multicolumn{5}{|c|}{ CSS (Jahre) } \\
\hline Patients at risk & $\mathbf{0}$ & $\mathbf{2 , 5}$ & $\mathbf{5}$ & $\mathbf{7 , 5}$ & $\mathbf{1 0}$ \\
\hline$\Sigma$ & 167 & 141 & 100 & 71 & 42 \\
\hline ypUICC-0 & 25 & 24 & 21 & 15 & 9 \\
\hline ypUICC-I & 38 & 32 & 27 & 19 & 11 \\
\hline ypUICC-II & 45 & 39 & 25 & 18 & 11 \\
\hline ypUICC-III & 44 & 35 & 24 & 17 & 10 \\
\hline ypUICC-IV & 15 & 11 & 3 & 2 & 1 \\
\hline
\end{tabular}

Abbildung 16: CSS in Abhängigkeit von den ypUICC-Stadien 0-IV

Postop. FU: postoperatives Follow up 


\subsection{4 ypUICC-Tumorstadien - Gesamtüberleben (OS)}

Das beschränkte mittlere (restricted mean) OS der 167 Patienten mit initial fortgeschrittenen Rektumkarzinomen beträgt über alle Tumorstadien (ypUICC-0 bis -IV) 105,86 Monate ( \pm 4,26 Monate; 95\%-Cl: 97,50-114,22 Monate, Tabelle 30). Wie es schon bei den anderen Überlebensparametern gesehen wurde, unterscheiden sich die K-M-Kurven für das OS in Abhängigkeit von den residuellen Tumorstadien signifikant (logrank-Test: $p<0,0009$ ). Patienten mit einer CR (ypUICC-0 Stadium) haben im OS sowohl nach Dauer (mittleres OS: 139,11 Monate \pm 4,28 Monate; 95\%-Cl: 130,73-147,50 Monate) als auch nach Anzahl der patients at risk (92\%) ein hohes Langzeitüberleben (Abbildung 17). In deutlichem Abstand dazu liegt das mittlere OS für Patienten im ypUICC-I Stadium bei 113,59 Monaten $\pm 8,28$ Monaten (95\%-Cl: 97,37-129,81 Monate). Das OS dieser Patienten liegt aber immer noch über den Werten der Gesamtpopulation (MW: 105,86 Monate \pm 4,26 Monate; 95\%-Cl: 97,50-114,22 Monate).

\begin{tabular}{|c|c|c|c|c|c|c|c|}
\hline \multirow{3}{*}{$\frac{\text { Stadium }}{\text { ypUICC-0 }}$} & \multirow{3}{*}{$\frac{N}{25}$} & \multirow{3}{*}{$\frac{\text { Ereignisse }}{2}$} & \multicolumn{4}{|c|}{ Überleben (OS, in Monaten) } & \multirow[b]{2}{*}{ logran $k^{* * *}$} \\
\hline & & & $\mathrm{rMW}^{*}$ & SD & \multicolumn{2}{|c|}{$95 \%$ Cl** } & \\
\hline & & & 139,11 & 4,28 & 130,73 & 147,50 & \multirow{6}{*}{$\mathrm{p}<0,0009$} \\
\hline ypUICC-I & 38 & 12 & 113,59 & 8,28 & 97,37 & 129,81 & \\
\hline ypUICC-II & 44 & 18 & 101,69 & 7,79 & 86,43 & 116,96 & \\
\hline ypUICC-III & 45 & 20 & 97,66 & 8,40 & 81,12 & 114,20 & \\
\hline ypUICC-IV & 15 & 11 & 53,40 & 10,47 & 32,89 & 73,91 & \\
\hline $\boldsymbol{\Sigma}$ & 167 & 63 & 105,86 & 4,26 & 97,50 & 114,22 & \\
\hline
\end{tabular}

Tabelle 30: OS (rMW) in Abhängigkeit von den ypUICC-Stadien 0-IV

$\mathrm{rMW}^{*}$ : beschränkte erwartete Überlebenszeit (restricted mean of OS) als Mittelwert für das Gesamtüberleben (OS); die Schätzung ist auf die längste Überlebenszeit begrenzt, wenn sie zensiert ist; SD: Standardabweichung; $95 \%-\mathrm{Cl}^{* *}$ : $95 \%$-Konfidenzintervall; Testung auf Gleichheit der Überlebensverteilung in der K-M-Darstellung für die ypUICCStadien: Chi-Quadrat 27,582, 4 Freiheitsgrade; logrank ${ }^{* \star *}$ : logrank-Test: $p<0,0009$

Die OS-Werte der ypUICC-II und ypUICC-III Stadien unterscheiden sich nur wenig und liegen unterhalb der rMW-Werte für das OS der Gesamtpopulation. Offensichtlich überwiegt bei diesen beiden Subgruppen die Co-Morbidität der Patienten und hat einen lebenslimitierenden Einfluss (Abschnitt 3.15). Mit der MMT wird für 
Patienten im Stadium ypUICC-IV ein deutlich schlechteres Überleben mit einem rMW des OS von 53,40 Monaten \pm 10,47 Monaten (95\%-Cl: 32,89-73,91 Monate) erreicht.

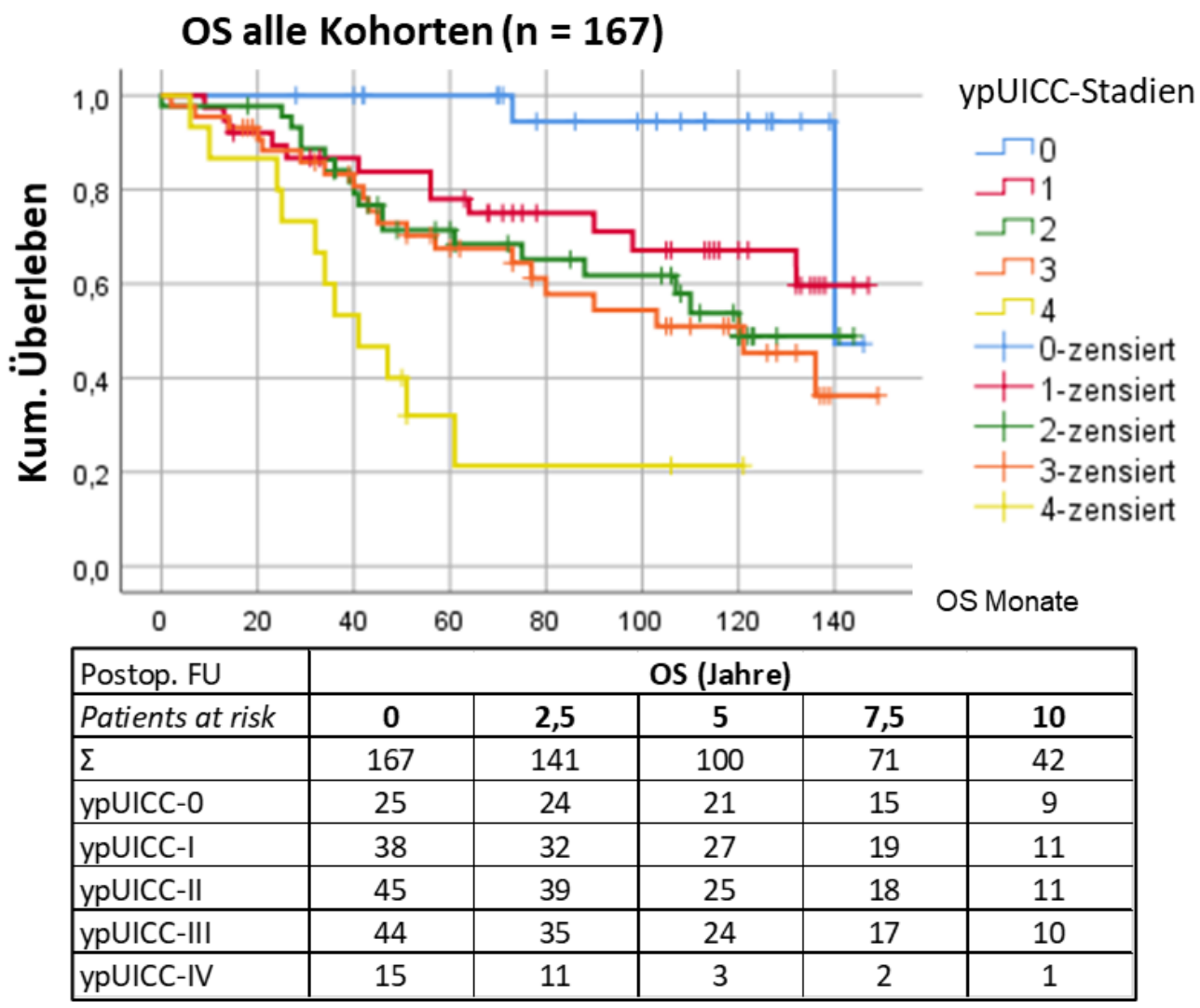

Abbildung 17: OS in Abhängigkeit von den ypUICC-Stadien 0-IV Postop. FU: postoperatives Follow up

\subsection{Univariate Survivalanalyse zum Tumoransprechen}

\subsubsection{Tumoransprechen - krankheitsfreies Überleben (DFS)}

Unabhängig vom Therapieregime zeigte sich für alle Patienten $(n=90$, Tabelle 31) mit einem GR-Status (TRG 3 und TRG 4) nach der präoperativen MMT ein signifikant (logrank-Test: $p=0,001$ ) besseres DFS (rMW für DFS: 105,35 Monate $\pm 6,3$ Monate, 95\%-Cl: 93,08-117,62 Monate) gegenüber Patienten mit PR-Status (TRG1 und TRG 2). Der rMW für das DFS von Patienten mit einer PR lag bei 72,75 Monaten (95\%-Cl: 59,97-85,53 Monate). 


\begin{tabular}{|c|c|c|c|c|c|c|c|}
\hline \multirow{3}{*}{$\begin{array}{l}\text { Response } \\
\text { GR (TRG } 3 \text { / 4) }\end{array}$} & \multirow{3}{*}{$\frac{N}{90}$} & \multirow{3}{*}{$\frac{\text { Ereignisse }}{32}$} & \multicolumn{4}{|c|}{ Überleben (DFS, in Monaten) } & \multirow[b]{2}{*}{ logrank*** } \\
\hline & & & $\mathrm{rMW}^{*}$ & SD & \multicolumn{2}{|c|}{$95 \% \mathrm{Cl}^{* *}$} & \\
\hline & & & 105,35 & 6,26 & 93,08 & 117,62 & \multirow{3}{*}{$p=0,001$} \\
\hline PR (TRG 1 / 2) & 77 & 46 & 72,75 & 6,52 & 59,97 & 85,53 & \\
\hline $\boldsymbol{\Sigma}$ & 167 & 78 & 91,47 & 4,79 & 82,08 & 100,86 & \\
\hline
\end{tabular}

Tabelle 31: DFS (rMW) in Abhängigkeit zum Tumoransprechen (gut vs. partiell)

GR: gutes TU-Ansprechen (good response, GR) umfasst alle Patienten mit TRG 4 und TRG 3 (nach Dworak et al. 1997); PR: partielles Ansprechen (partial response), betrifft Patienten mit TRG 1 und TRG 2; rMW*: beschränkte erwartete Überlebenszeit (restricted mean of DFS) als Mittelwert für das DFS, diese Schätzung ist auf die längste Überlebenszeit begrenzt, wenn sie zensiert wurde; SD: Standardabweichung; $95 \%-\mathrm{Cl}^{\star *}$ : $95 \%-$ Konfidenzintervall; Testung auf Gleichheit der Überlebensverteilung in der Kaplan-MeierDarstellung für das unterschiedliche Tumoransprechen: Chi-Quadrat 11,05, 1 Freiheitsgrad; logrank ${ }^{* \star}$ : logrank-Test: $p=0,001$

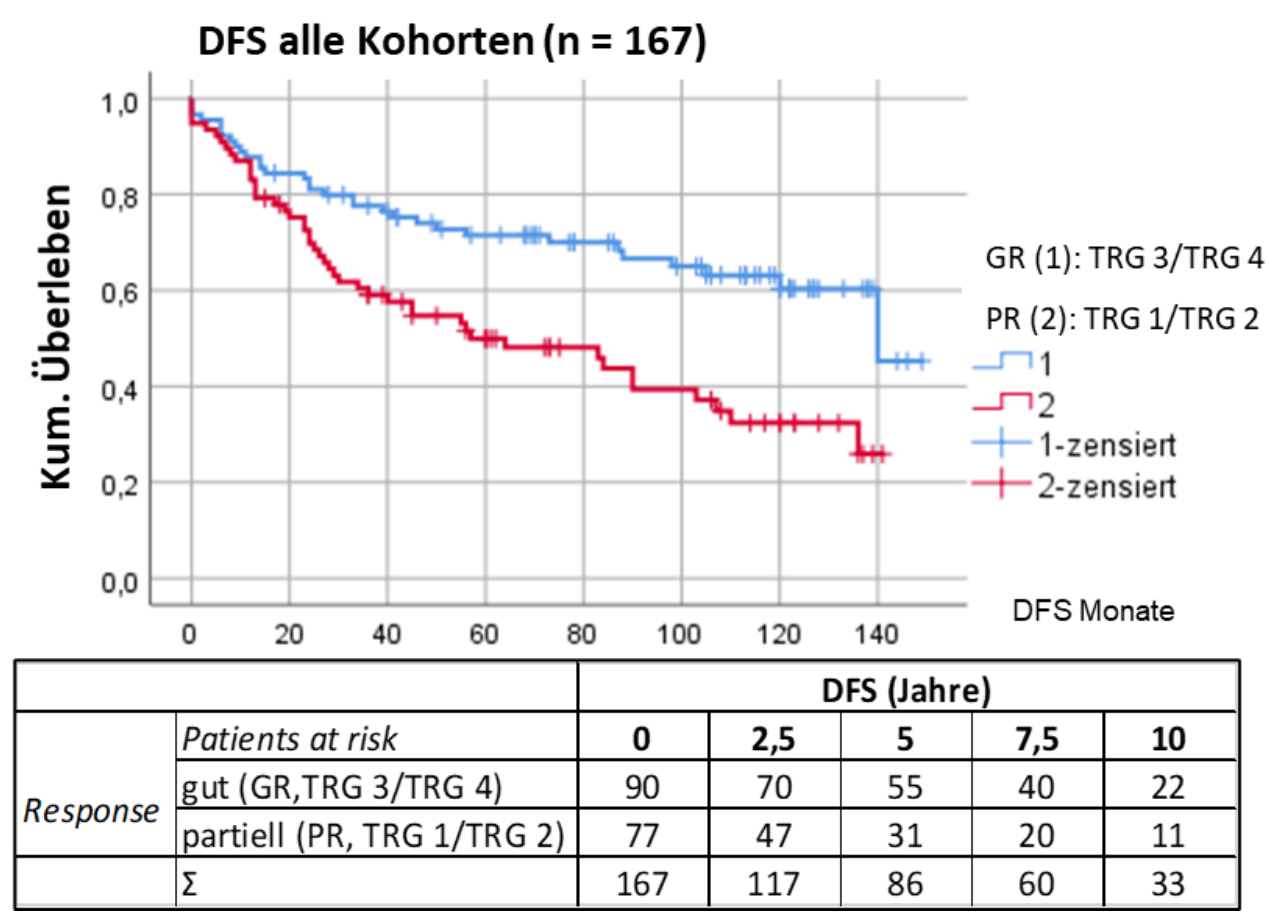

Abbildung 18: DFS in Abhängigkeit zum Tumoransprechen (gut vs. partiell) 


\subsubsection{Tumoransprechen - krebsspezifisches Überleben (CSS)}

Für das krebsspezifische Überleben (CSS) zeigt sich abweichend vom DFS und RFS (nicht dargestellt) ab dem 40. Monat post operationem nur ein tendenzieller Überlebensvorteil für Patienten mit einem GR-Status. Trotz des höheren 95\%-Cl (118,40-137,77 Monate versus 97,59-121,80 Monate) im CSS besteht für die Patienten mit GR im univariaten Vergleich kein signifikanter Überlebensvorteil (logrank-Test: $p=0,062$ ).

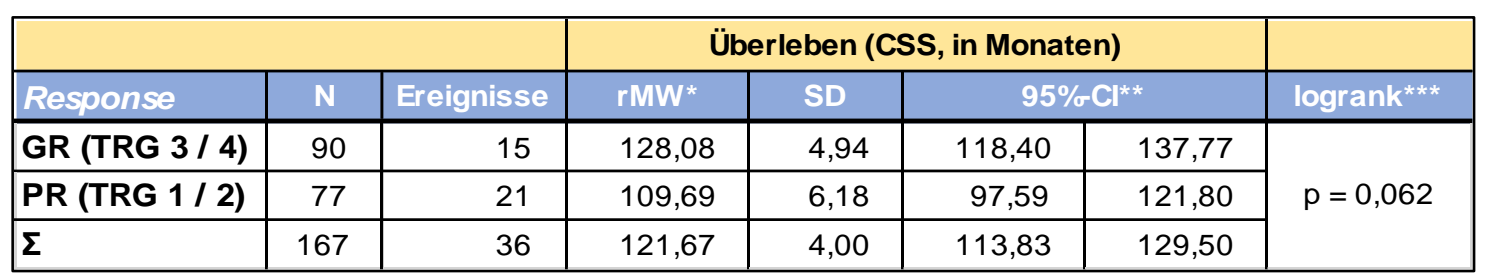

Tabelle 32: CSS (rMW) in Abhängigkeit zum Tumoransprechen (gut vs. partiell) GR: gutes TU-Ansprechen (good response), Patienten einer Tumorremission TRG 4 und TRG 3 (nach Dworak et al. 1997); PR: partielles Ansprechen (partial response), Patienten mit einem TRG 1 und TRG 2; rMW*: beschränkte erwartete Überlebenszeit (restricted mean of CSS) als Mittelwert für das CSS, diese Schätzung ist auf die längste Überlebenszeit begrenzt, wenn sie zensiert wurde; SD: Standardabweichung; $95 \%-\mathrm{Cl}^{\star *}$ : $95 \%-$ Konfidenzintervall; Testung auf Gleichheit der Überlebensverteilung in der K-MDarstellung für das Tumoransprechen: Chi-Quadrat 3,486, 1 Freiheitsgrad; logrank ${ }^{* \star *}$ : logrank-Test: $p=0,062$

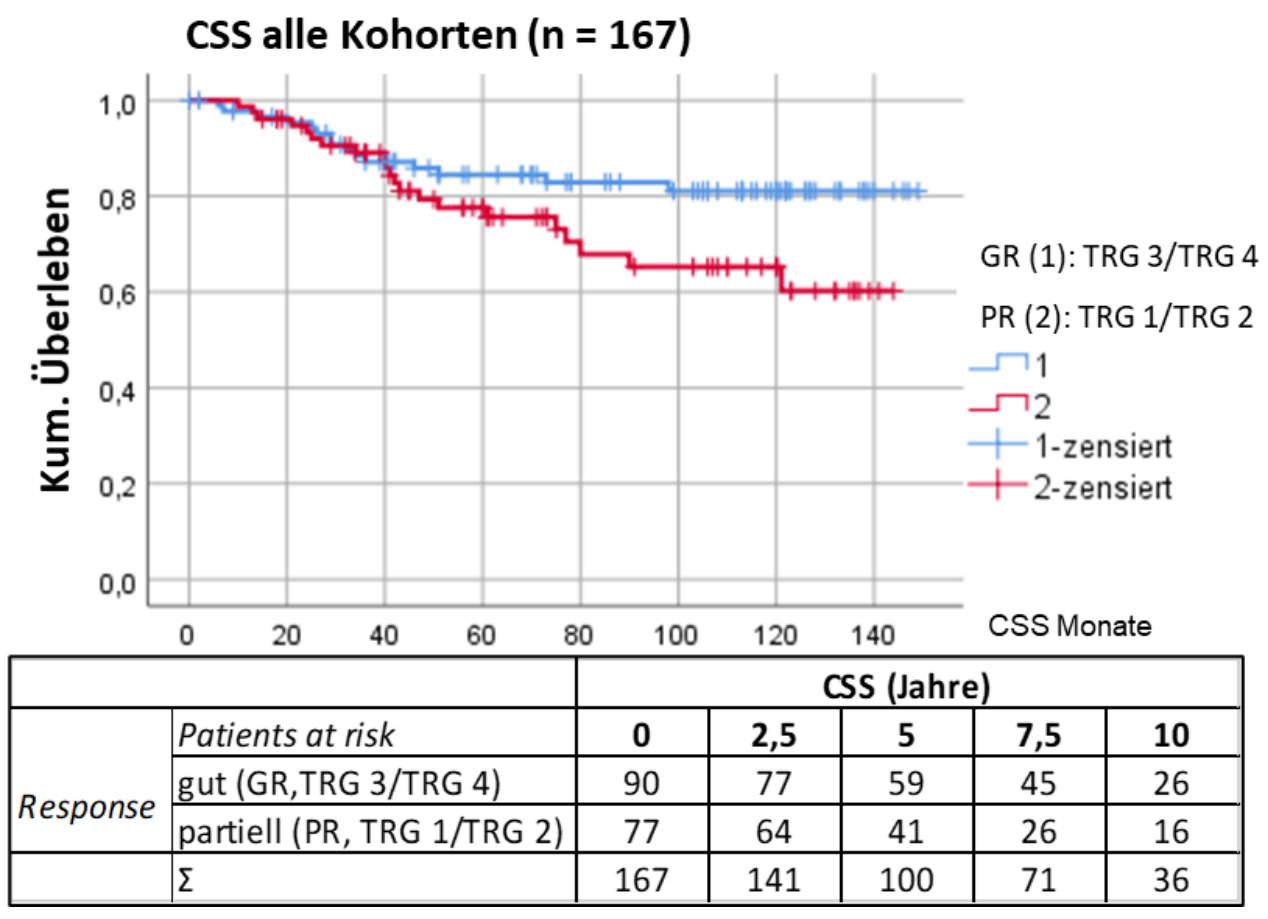

Abbildung 19: CSS in Abhängigkeit zum Tumoransprechen (gut vs. partiell) 


\subsubsection{Tumoransprechen - Gesamtüberleben}

Über den gesamten Beobachtungszeitraum haben Patienten $(n=90)$ mit einem guten Ansprechen des Tumors (GR) auf die MMT ein signifikant (logrank-Test: $p=0,007$ ) besseres OS (rMW für OS: 115,03 Monate $\pm 5,66$ Monate; $95 \%-C l$ : 103,94-126,13 Monate) gegenüber Patienten $(n=77)$ mit einem partiellen Tumoransprechen (PR). Allein in den ersten 24 Monaten der Nachbeobachtung scheinen Patienten mit einer PR in den K-M-Verlaufskurven und anhand der patients at risk Daten ein nahezu gleiches OS gegenüber den Patienten mit einer GR zu haben. Aus dieser Beobachtung lässt sich folgern, dass längere Nachbeobachtungszeiten (> 36 Monate) notwendig sind, um die aufgrund eines Tumoransprechens erst verzögert auftretenden Rezidivereignisse adäquat erfassen und zuordnen zu können.

\begin{tabular}{|c|c|c|c|c|c|c|c|}
\hline \multirow{3}{*}{$\frac{\text { Response }}{\text { GR (TRG } 3 \text { / 4) }}$} & \multirow{3}{*}{$\frac{N}{90}$} & \multirow{3}{*}{$\frac{\text { Ereignisse }}{26}$} & \multicolumn{4}{|c|}{ Überleben (OS, in Monaten) } & \multirow[b]{2}{*}{ logrank ${ }^{* * *}$} \\
\hline & & & rMW* & SD & \multicolumn{2}{|c|}{$95 \% \mathrm{Cl}^{* *}$} & \\
\hline & & & 115,03 & 5,66 & 103,94 & 126,13 & \multirow{3}{*}{$p=0,007$} \\
\hline PR (TRG 1 / 2) & 77 & 37 & 92,80 & 5,96 & 81,12 & 104,48 & \\
\hline $\boldsymbol{\Sigma}$ & 167 & 63 & 105,86 & 4,26 & 97,50 & 114,22 & \\
\hline
\end{tabular}

Tabelle 33: OS (rMW) in Abhängigkeit zum Tumoransprechen (gut vs. partiell)

OS: Gesamtüberleben; GR: gutes TU-Ansprechen (good response), umfasst Patienten mit einer Tumorremission TRG 4 und TRG 3 (nach Dworak et al. 1997) im Rektumresektat; PR: partielles Ansprechen (partial response), betrifft Patienten mit TRG 1 und TRG 2 nach präoperativer Multimodaltherapie. $\mathrm{rMW}^{*}$ : beschränkte erwartete Überlebenszeit (restricted mean of survival) als Mittelwert für das OS, diese Schätzung ist auf die längste Überlebenszeit begrenzt, wenn sie zensiert wurde; SD: Standardabweichung; $95 \%-\mathrm{Cl}^{* *}$ : 95\%-Konfidenzintervall; Testung auf Gleichheit der Überlebensverteilung in der KaplanMeier-Darstellung für das unterschiedliche Tumoransprechen: Chi-Quadrat 7,188, 1 Freiheitsgrad; logrank ${ }^{\star * *}$ : logrank-Test: $p=0,007$ 


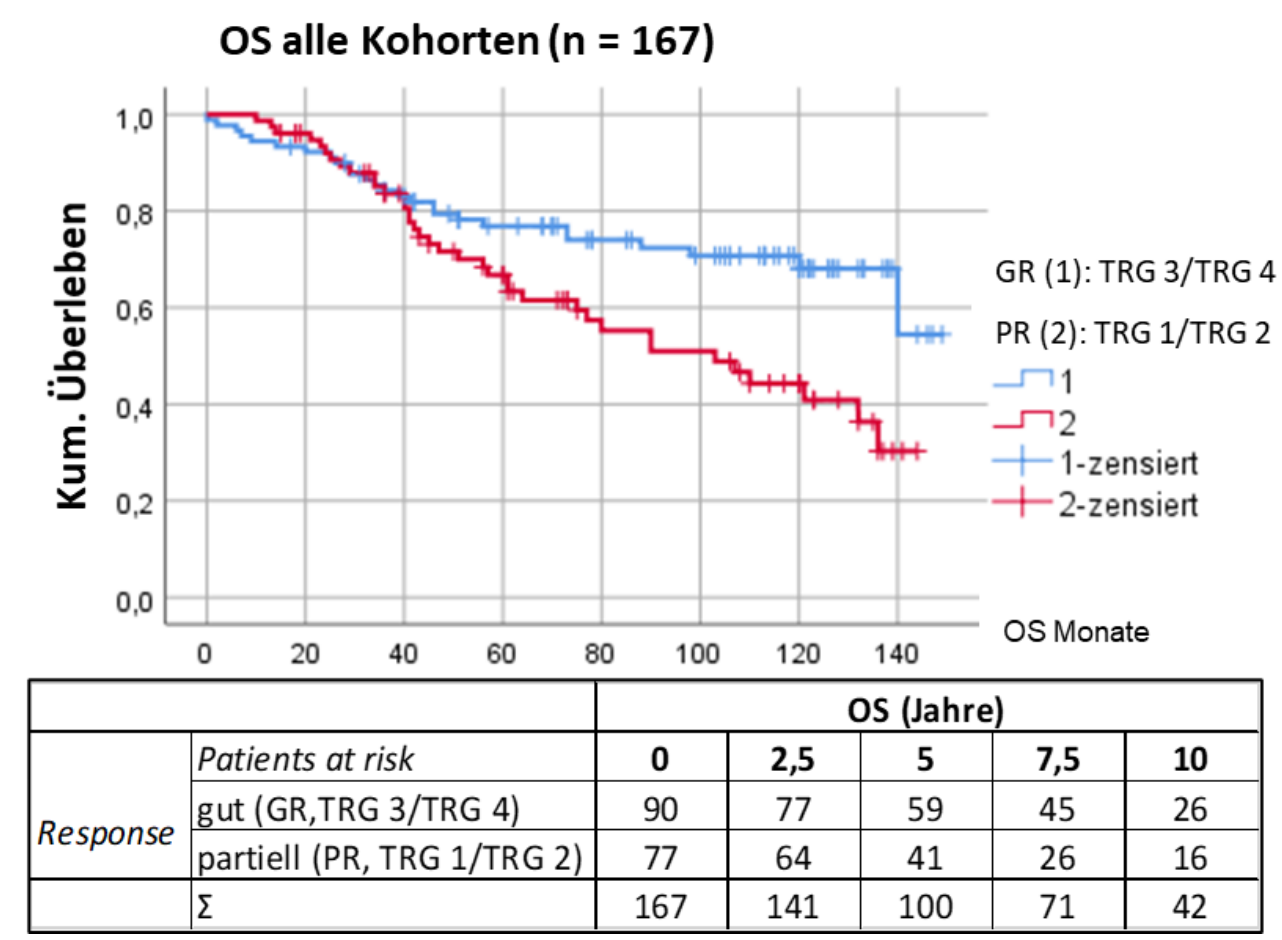

Abbildung 20: OS in Abhängigkeit zum Tumoransprechen (gut vs. partiell)

\subsection{Univariate Survivalanalyse zum ypL-/V-/Pn-Status}

\subsection{1 ypL-/V-/Pn-Status - krankheitsfreies Überleben (DFS)}

Analog der zunehmenden Bedeutung einer in der MRT erkennbaren extramuralen Gefäßinfiltration (extramural vascular invasion, EMVI) bei Rektumkarzinomen wurde in der Studienpopulation die Bedeutung eines positiven ypL-/V-/Pn-Status geprüft. Interessanterweise zeigten Patienten $(n=121)$ mit einem negativen ypL-/V-/Pn-Status in der univariaten Analyse, unabhängig vom Tumorstadium und anderen Parametern, eine signifikant besseres DFS (rMW für DFS: 100,25 Monate $\pm 5,26$ Monate; 95\%-Cl: 89,94-110,56 Monate) gegenüber Patienten $(n=46)$ mit einem positiven ypL-/V-/Pn-Status (logrank-Test: $p=0,003$ ). Bei letzteren Patienten betrug das DFS im Mittel 65,06 Monate \pm 8,94 Monate (Tabelle 34). Das 95\%-Cl lag mit 47,54 bis 82,58 Monaten unter dem Minimalwert des 95\%-Cl jener Studienteilnehmer mit unauffälligem ypL-/V-/Pn-Status. Dieses Ergebnis lässt sich auch aus dem Verlauf der K-M-Kurve ablesen, allerdings fällt auf, dass nach ca. 
70-monatiger Nachbeobachtungszeit das DFS in beiden Subkohorten von anderen Co-Faktoren beeinflusst zu sein scheint.

\begin{tabular}{|c|c|c|c|c|c|c|c|}
\hline \multirow{3}{*}{$\frac{\text { ypL-/V-/Pn-Status }}{\text { ypL-/V-/Pn-negativ }}$} & \multirow{3}{*}{$\frac{N}{121}$} & \multirow{3}{*}{$\frac{\text { Ereignisse }}{50}$} & \multicolumn{4}{|c|}{ Überleben (DFS, in Monaten) } & \multirow[b]{2}{*}{$\log r a n k^{* * *}$} \\
\hline & & & rMW* & SD & \multicolumn{2}{|c|}{$95 \% \mathrm{Cl}^{* *}$} & \\
\hline & & & 100,25 & 5,26 & 89,94 & 110,56 & \multirow{3}{*}{$p=0,003$} \\
\hline ypL-/V-/Pn-positiv & 46 & 28 & 65,06 & 8,94 & 47,54 & 82,58 & \\
\hline $\boldsymbol{\Sigma}$ & 167 & 78 & 91,47 & 4,79 & 82,08 & 100,86 & \\
\hline
\end{tabular}

Tabelle 34: DFS (rMW) der Patienten in Abhängigkeit zum ypL-/V-/Pn-Status ypL-/V-/Pn-negativ: kein histopathologischer Nachweis einer Lymphangiosis carcinomatosa (L), keine karzinomatöse Infiltration in Gefäße (V) und/oder Perineuralscheiden (Pn) nach präoperativer MMT; ypL-/V-/Pn-positiv: histopathologischer ( $p$ ) Nachweis einer verbliebenen Karzinominfiltration in den Tumor-begleitenden Lymphgefäßen (L1) und/oder Blutgefäßen (V1) und/oder Perineuralscheiden (Pn1) nach präoperativer CRT +/- CTx (y); $\mathrm{rMW}^{*}$ : beschränkte erwartete Überlebenszeit (restricted mean of survival) als Mittelwert für das DFS, diese Schätzung ist auf die längste Überlebenszeit begrenzt, wenn sie zensiert wurde; SD: Standardabweichung; 95\%-Cl**: 95\%-Konfidenzintervall; Testung auf Gleichheit der Überlebensverteilung in der Kaplan-Meier-Darstellung für den ypL-/V-/PnStatus: Chi-Quadrat 8,553, 1 Freiheitsgrad; logrank ${ }^{* * *}$ : logrank-Test: $p=0,003$

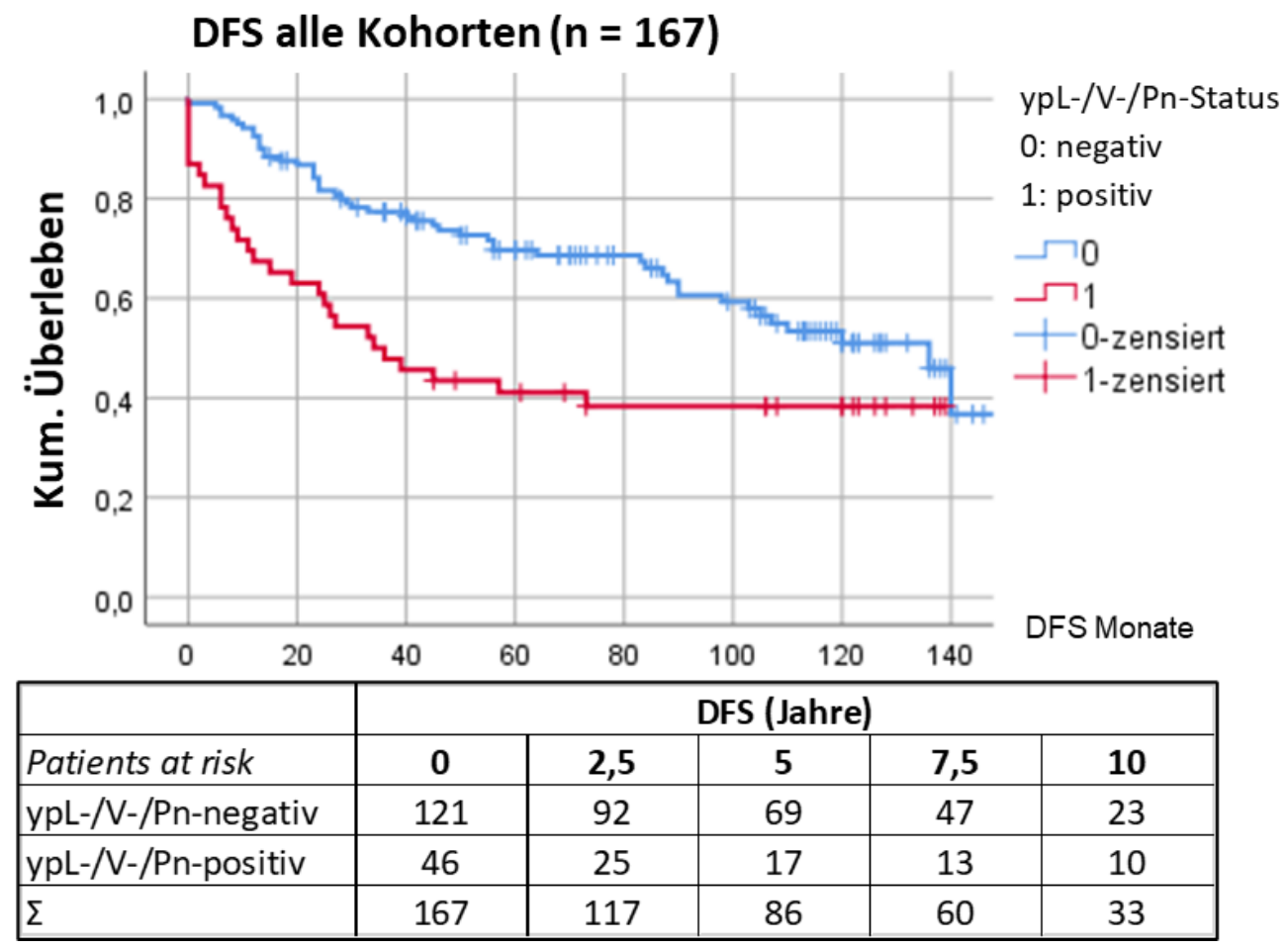

Abbildung 21: DFS in Abhängigkeit zum ypL-/V-/Pn-Status 


\subsection{2 ypL-/V-/Pn-Status - krebsspezifisches Überleben (CSS)}

Ähnlich wie beim DFS haben Patienten mit einem negativen ypL-/V-/Pn-Status $(n=121)$ ein signifikant (logrank-Test: $p=0,008$ ) längeres krebsspezifisches Überleben (rMW für CSS: 127,97 Monate \pm 4,1 Monate; 95\%-Cl: 119,86-136,31 Monate) gegenüber Patienten $(n=46)$ mit einem positiven ypL-/V-/Pn-Status (rMW für CSS: 97,56 Monate \pm 8,56 Monate; 95\%-Cl: 80,79-114,33 Monate, Tabelle 35). Wie es bereits in den K-M-Überlebenskurven zum DFS vermutet wurde, zeigen die CSS-Kurven ab dem 70. Nachbeobachtungsmonat einen annähernd parallelen Verlauf. Auch hier scheinen Rektumkarzinom-unabhängige Co-Faktoren für eine Reduktion des Überlebens der Patienten verantwortlich gewesen zu sein.

\begin{tabular}{|c|c|c|c|c|c|c|c|}
\hline \multirow{3}{*}{$\frac{\text { ypL-/V-/Pn-Status }}{\text { ypL-/V-/Pn-negativ }}$} & \multirow{3}{*}{$\frac{N}{121}$} & \multirow{3}{*}{$\frac{\text { Ereignisse }}{21}$} & \multicolumn{4}{|c|}{ Überleben (CSS, in Monaten) } & \multirow[b]{2}{*}{ logrank*** } \\
\hline & & & rMW* & SD & \multicolumn{2}{|c|}{$95 \% \mathrm{Cl}^{* *}$} & \\
\hline & & & 127,97 & 4,14 & 119,86 & 136,08 & \multirow{3}{*}{$p=0,008$} \\
\hline ypL-/V-/Pn-positiv & 46 & 15 & 97,56 & 8,56 & 80,79 & 114,33 & \\
\hline $\boldsymbol{\Sigma}$ & 167 & 36 & 121,67 & 4,00 & 113,83 & 129,50 & \\
\hline
\end{tabular}

Tabelle 35: CSS der Patienten in Abhängigkeit zum ypL-/V-/Pn-Status

ypL-/V-/Pn-negativ: kein histopathologischer Nachweis einer Lymphangiosis carcinomatosa (L), keine karzinomatöse Infiltration in Gefäße (V) und/oder Perineuralscheiden (Pn) nach präoperativer MMT; ypL-/V-/Pn-positiv: histopathologischer (p) Nachweis einer Karzinominfiltration in Lymph- (L1) und/oder Blutgefäße (V1) und/oder Perineuralscheiden (Pn1) nach präoperativer CRT +/- CTx (y); rMW*: beschränkte erwartete Überlebenszeit als Mittelwert (restricted mean of CSS), diese Schätzung ist auf die längste Überlebenszeit begrenzt, wenn sie zensiert wurde; SD: Standardabweichung; $95 \%-\mathrm{Cl}^{* *}$ : $95 \%-$ Konfidenzintervall; Testung auf Gleichheit der Überlebensverteilung in der Kaplan-MeierDarstellung für den ypL-/V-/Pn-Status: Chi-Quadrat 7,111, 1 Freiheitsgrad; logrank ${ }^{\star \star *}$ : logrank-Test: $p=0,008$ 


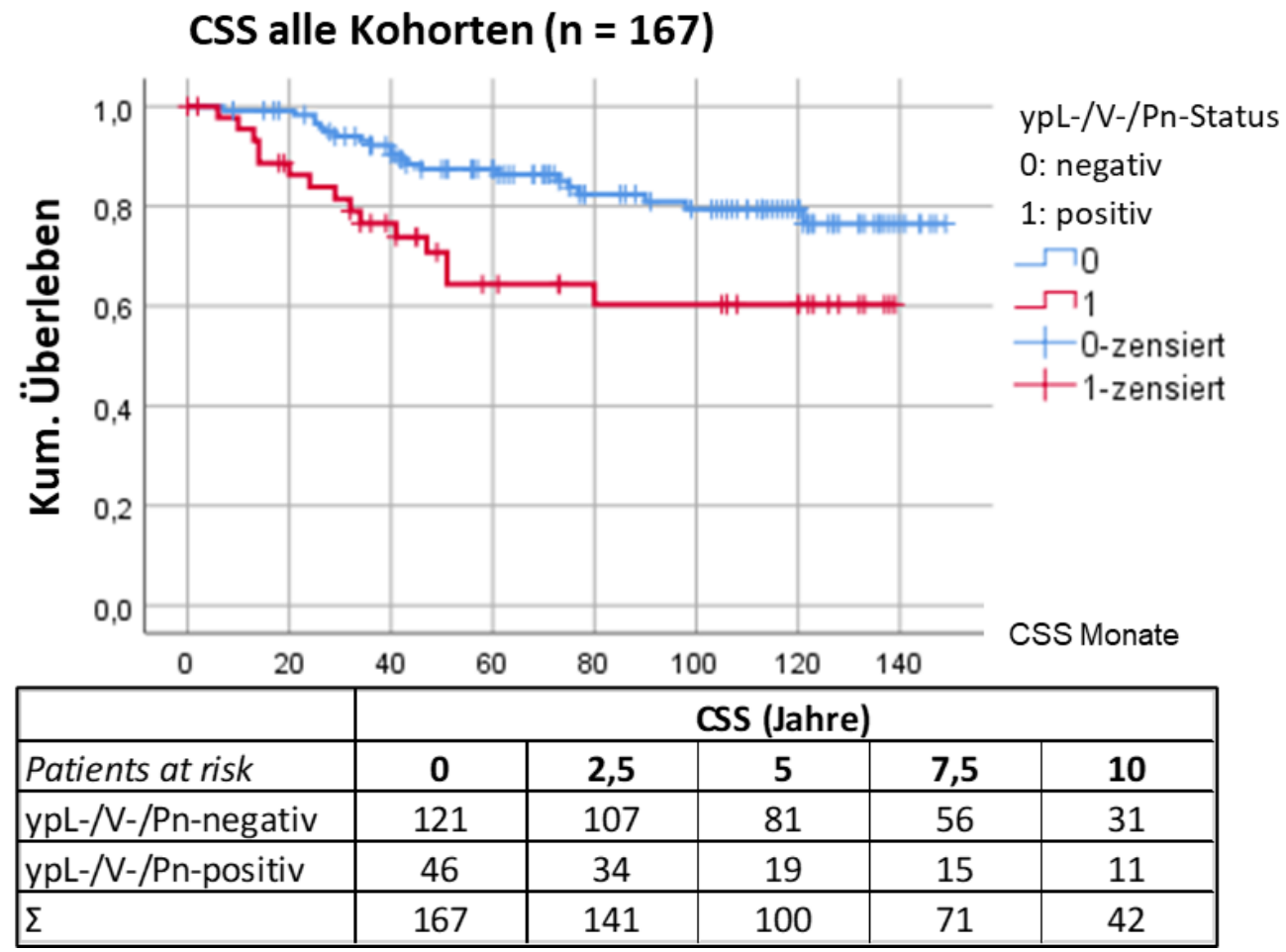

Abbildung 22: CSS in Abhängigkeit zum ypL-/V-/Pn-Status

\subsection{3 ypL-/V-/Pn-Status - Gesamtüberleben (OS)}

Der bereits beim DFS und CSS beschriebene, signifikante Unterschied in den K-M-Überlebenskurven findet sich auch beim OS (logrank-Test: $p=0,003$ ). Die OS-Kurven der Patienten mit einem negativen bzw. positiven ypL-/V-/Pn-Status verlaufen analog der DFS-Kurven mit einer Verschiebung auf der Zeitachse (x-Achse) um 14 Monate (rMW des DFS: 91,5 Monate vs. rMW des OS: 105,9 Monate bei Patienten mit ypL-/V-/Pn-Status). Die K-M-Kurven für das OS liegen bei beiden Gruppen am Beobachtungsende bei knapp über 40 \%. Beim Vergleich der Kurvenverläufe zeigt sich jedoch ein schnelleres Absinken der K-M-Kurven für EMVI-positive Patienten im Gegensatz zu den EMVI-negativen Fällen. Für die EMVI-positiven Patienten ist bereits bei 60 Monaten (roter Kreis, Abbildung 23) das mediane OS erreicht gegenüber 140 Monaten (blauer Kreis, Abbildung 23) bei EMVI-negativen Patienten. Dieser gegenüber dem CSS beobachtete Kurvenabfall kann als Hinweis auf lebenslimitierende Nebenerkrankungen gewertet werden. Der flachere Kurvenverlauf bei EMVI-negativen Patienten lässt einen relativen Überlebensvorteil dieser Patientengruppe vermuten. 


\begin{tabular}{|c|c|c|c|c|c|c|c|}
\hline \multirow{3}{*}{$\frac{\text { ypL-/V-/Pn-Status }}{\text { ypL-/V-/Pn-negativ }}$} & \multirow{3}{*}{$\frac{N}{121}$} & \multirow{3}{*}{$\frac{\text { Ereignisse }}{39}$} & \multicolumn{4}{|c|}{ Überleben (OS, in Monaten) } & \multirow[b]{2}{*}{ logrank $k^{* * *}$} \\
\hline & & & $r M W^{*}$ & SD & \multicolumn{2}{|c|}{$95 \% \mathrm{Cl}^{* *}$} & \\
\hline & & & 114,20 & 4,53 & 105,32 & 123,08 & \multirow{3}{*}{$p=0,003$} \\
\hline ypL-/V-/Pn-positiv & 46 & 24 & 79,79 & 8,31 & 63,50 & 96,07 & \\
\hline $\boldsymbol{\Sigma}$ & 167 & 63 & 105,86 & 4,26 & 97,50 & 114,22 & \\
\hline
\end{tabular}

Tabelle 36: OS (rMW) der Patienten in Abhängigkeit zum ypL-/V-/Pn-Status

ypL-/V-/Pn-negativ: kein histopathologischer Nachweis einer Lymphangiosis carcinomatosa (L), keine karzinomatöse Infiltration in Gefäße (V) und/oder Perineuralscheiden (Pn) nach präoperativer CRT +/- CTx (y); ypL-/V-/Pn-positiv: histopathologischer (p) Nachweis einer Karzinominfiltration in tumorbegleitenden Lymph- (L1) und/oder Blutgefäßen (V1) und/oder Perineuralscheiden (Pn1); rMW*: beschränktes erwartetes Gesamtüberleben als Mittelwert (restricted mean of OS), diese Schätzung ist auf die längste Überlebenszeit begrenzt, wenn sie zensiert wurde; SD: Standardabweichung; 95\%-Cl** : 95\%-Konfidenzintervall; Testung auf Gleichheit der Überlebensverteilung in der Kaplan-Meier-Darstellung für den ypL-/V-/Pn-Status: Chi-Quadrat 9,140, 1 Freiheitsgrad; logrank ${ }^{\star * *}$ : logrank-Test: $p=0,003$

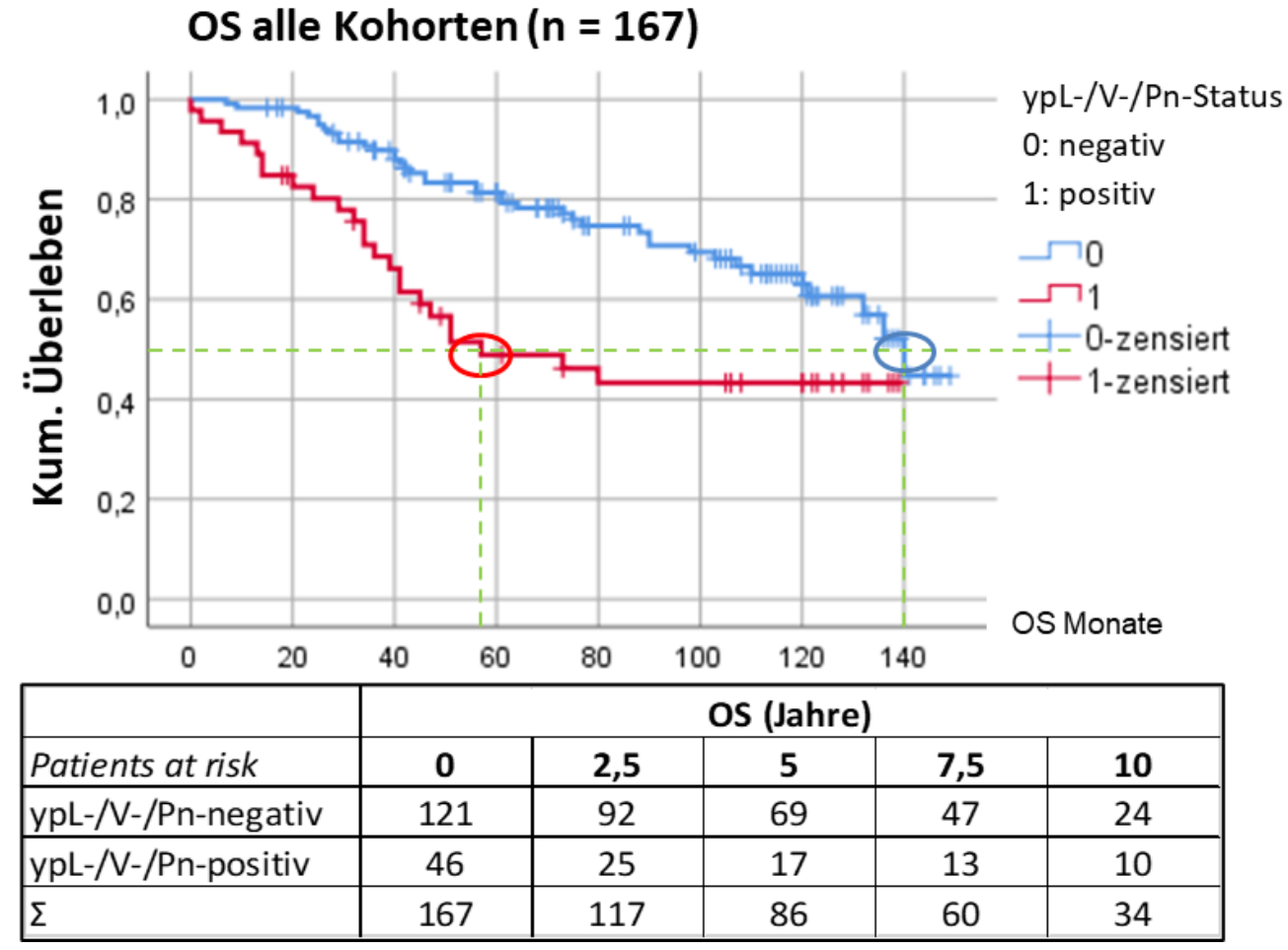

Abbildung 23: OS in Abhängigkeit des ypL-/V-/Pn-Status

Roter Kreis: mediane Überlebenswahrscheinlichkeit der Patienten mit positivem ypL-/V-/Pn-Status; blauer Kreis: mediane Überlebenswahrscheinlichkeit der Patienten mit negativem ypL-/V-/Pn-Status 


\subsection{Weitere Parameter nach MMT mit Einfluss auf das Überleben}

In der Tabelle 37 werden die residuelle Tumorlängsausdehnung (урTE) und der zirkumferentielle Resektionsrand (ypCRM) als weitere Parameter nach der MMT dargestellt, die in der univariaten Analyse Einfluss auf die Überlebensparameter DFS, CSS und OS hatten. Für beide Parameter zeigten sich signifikante Unterschiede in den K-M-Überlebenskurven der Patienten mit ypTE $\leq 25 \mathrm{~mm}$ vs. ypTE $>25 \mathrm{~mm}$ und ypCRM $\geq 2 \mathrm{~mm}$ vs. ypCRM $<2 \mathrm{~mm}$ (K-M-Kurven sind nicht dargestellt).

\begin{tabular}{|c|c|c|c|c|c|c|}
\hline & DFS & css & os & DFS & css & os \\
\hline & \multicolumn{3}{|c|}{ ypTE $\leq 25 \mathrm{~mm}$} & \multicolumn{3}{|c|}{ ypCRM $\geq 2 \mathrm{~mm}$} \\
\hline $\mathrm{N}$ & 92 & 92 & 92 & 153 & 153 & 153 \\
\hline Ereignisse & 35 & 13 & 26 & 68 & 29 & 54 \\
\hline $\mathrm{rMW}^{*}$ & 100,99 & 130,93 & 115,75 & 95,03 & 125,37 & 109,32 \\
\hline SD & 6,39 & 4,62 & 5,45 & 4,90 & 3,92 & 4,31 \\
\hline \multirow[t]{2}{*}{$95 \%-\mathrm{Cl}^{\star *}$} & $88,46-113,52$ & $121,89-139,98$ & $105,07-126,43$ & $85,44-104,63$ & $117,70-133,05$ & $100,87-117,77$ \\
\hline & \multicolumn{3}{|c|}{ УРТЕ > 25 mm } & \multicolumn{3}{|c|}{ ypCRM < 2 mm } \\
\hline $\mathrm{N}$ & 75 & 75 & 75 & 14 & 14 & 14 \\
\hline Ereignisse & 43 & 23 & 37 & 10 & 7 & 9 \\
\hline $\mathrm{rMW}^{*}$ & 78,12 & 107,56 & 92,31 & 49,07 & 75,07 & 64,29 \\
\hline SD & 6,60 & 6,26 & 6,17 & 15,41 & 15,91 & 14,31 \\
\hline \multirow[t]{2}{*}{$95 \%-\mathrm{Cl}^{\star *}$} & $65,18-91,07$ & $95,28-119,83$ & $80,22-104,40$ & $18,87-79,27$ & $43,88-106,26$ & $36,25-92,34$ \\
\hline & \multicolumn{3}{|c|}{ gesamt } & \multicolumn{3}{|c|}{ gesamt } \\
\hline $\mathrm{N}$ & 167 & 167 & 167 & 167 & 167 & 167 \\
\hline Ereignisse & 78 & 36 & 63 & 78 & 36 & 63 \\
\hline $\mathrm{rMW}^{*}$ & 91,47 & 121,67 & 100,86 & 91,47 & 121,67 & 105,86 \\
\hline SD & 4,79 & 4,00 & 4,26 & 4,79 & 4,00 & 4,26 \\
\hline $95 \%-C l^{\star *}$ & $82,08-100,86$ & $113,83-129,50$ & $97,50-114,22$ & $82,08-100,86$ & $113,83-129,50$ & $97,50-114,22$ \\
\hline $\operatorname{logrank}^{\star \star \star}$ & $p=0,023$ & $p=0,011$ & $p=0,007$ & $p=0,002$ & $p<0,0009$ & $p=0,001$ \\
\hline
\end{tabular}

Tabelle 37: Überleben (rMW) in Abhängigkeit zum ypTE- und ypCRM-Status

ypTE $\leq 25 \mathrm{~mm}$ : Patienten mit einer Tumorlängsausdehnung von $\leq 25 \mathrm{~mm}$ (Medianwert der Gesamtpopulation) nach präoperativer Multimodaltherapie; ypTE $>25 \mathrm{~mm}$ : Patienten mit einer residuellen Tumorlängsausdehnung von $>25 \mathrm{~mm}$; ypCRM $\geq 2 \mathrm{~mm}$ : Patienten mit einem histopathologisch überprüften tumorfreien zirkumferentiellen Sicherheitsabstand von $\geq 2 \mathrm{~mm}$ bis zur chirurgischen Resektionsebene; ypCRM $<2 \mathrm{~mm}$ : Patienten mit einem tumorfreien CRM von $<2 \mathrm{~mm}$ in der postoperativen histopathologischen Resektatbeurteilung; $\mathrm{rMW}^{*}$ : beschränktes erwartetes Überleben (restricted mean of survival) als Mittelwerte für das DFS, CSS oder OS; die Schätzungen sind auf die längste Überlebenszeit begrenzt, wenn sie zensiert wurden; SD: Standardabweichung; 95\%-Cl**: 95\%Konfidenzintervall; Testung auf Gleichheit der Überlebensverteilung; logrank ${ }^{* * *}$ : logrankTest

In der histopathologischen Untersuchung der Rektumresektate nach präoperativer MMT waren bei nur wenigen Patienten $(n=14)$ Tumorzellen im unmittelbaren Ab- 
stand zum zirkumferentiellen Resektionsrand (ypCRM: $<2 \mathrm{~mm}$ ) nachweisbar gewesen.

\subsection{Subgruppenanalysen}

Nach der präoperativen Multimodaltherapie wurde bei 107 Patienten der Kohorten A und B eine adCTx eingeleitet und von 106 Patienten beendet (Abbildung 24). Im Folgenden wird über Subgruppen-Analysen versucht, die prognostische Bedeutung des Tumoransprechens auf die MMT zu klären.

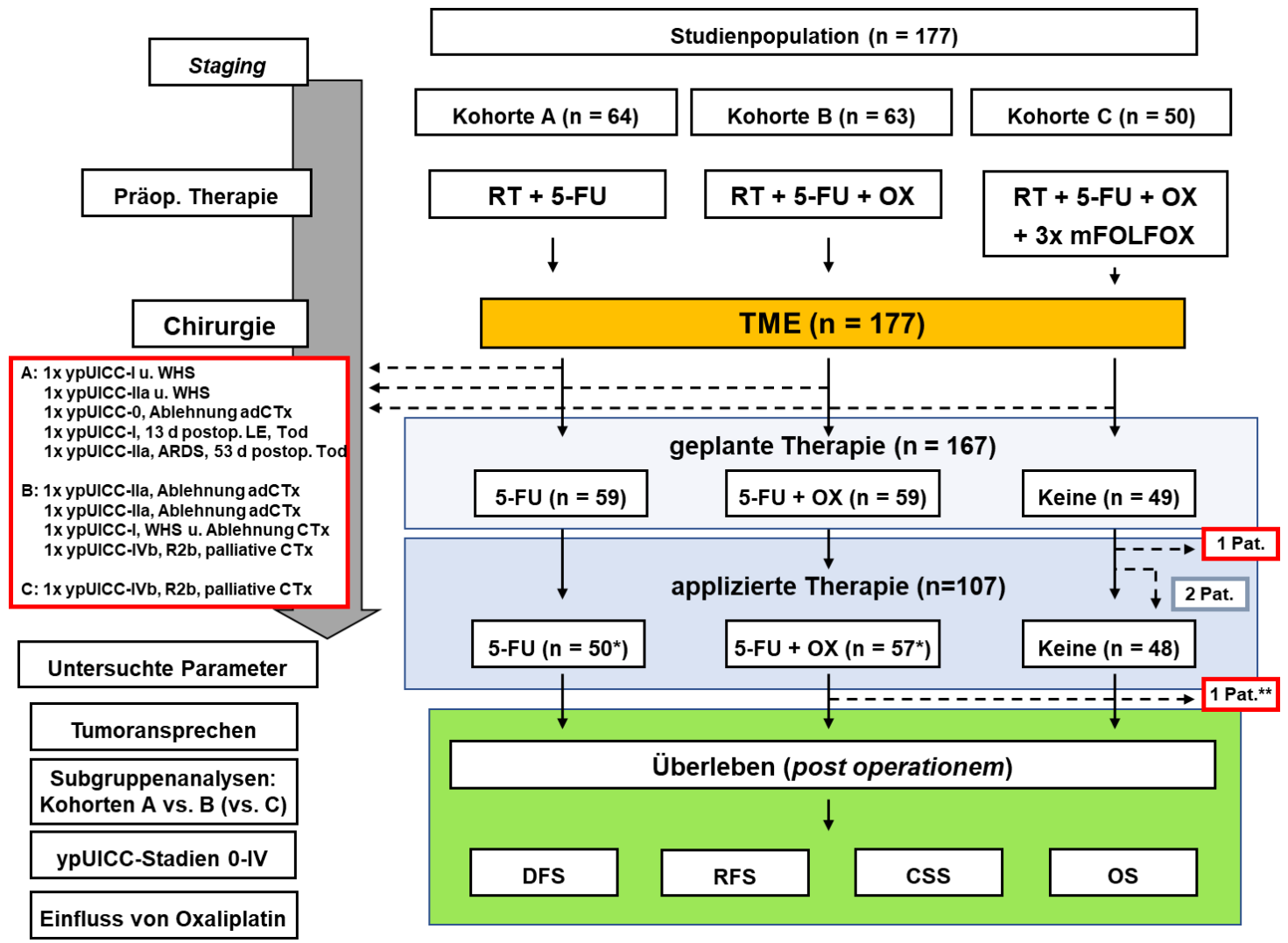

Abbildung 24: Studienpopulation und Auswertungsparameter (II) nach MMT

Eigene Darstellung; *: Patientenkohorten $A(n=50)$ und $B(n=57)$ ohne jene neun Patienten, die postoperativ die adCTx wechselten (9x Kohorte A nach B, 2x Kohorte B nach A). In Kohorte $C(n=48)$ sind jene 2 Patienten ausgeschlossen, die nach der kompletten präoperativer MMT einen ypR2b-Status aufwiesen. Nach der totalen mesorektalen Exzision (TME) hatten 107 Patienten der Kohorten A und B die geplante adCTx begonnen. Diese adCTx wurde von 106 Patienten beendet. Es trat ${ }^{* *} 1$ Todesfall in Kohorte B während der postoperativen Therapiephase aufgrund eines Sick-Sinus-Syndroms mit kardialer Dekompensation ein. Zwei Patienten (blau umrahmt) der Kohorte $\mathrm{C}$ erhielten als Einzelfallentscheidung eine limitierte adCTx mit FOLFOX. Insgesamt wurden 155 Patienten (Kohorte A: $n=50$, Kohorte B: $n=57$, Kohorte C: $n=48$ ) post operationem beobachtet. 


\subsubsection{Tumoransprechen - postoperative Therapie (as-treated)}

In der univariaten Auswertung der 167 Patienten, die nach der TME ihrer geplanten adCTx (Kohorten A und B) oder alleinigen Nachsorge (Kohorte C) zugeführt werden sollten (Abbildung 24), ergaben sich für Patienten mit TRG 3 und TRG 4 Status ein besseres DFS (logrank-Test: $p=0,001$; Abschnitt 3.11.1) und RFS (Iogrank-Test: $p=0,001$ ), unabhängig vom UICC-Status und der Tumorgröße.

\begin{tabular}{|c|c|c|c|c|c|}
\hline Ansprechen* & ypUICC-Stadium & TRG & $\mathbf{N}$ & $\Sigma$ Pati & nten \\
\hline \multirow{4}{*}{ gut } & ypUICC-0 (урTON0) & TRG 4 & 25 & \multirow{4}{*}{68} & \multirow{24}{*}{ 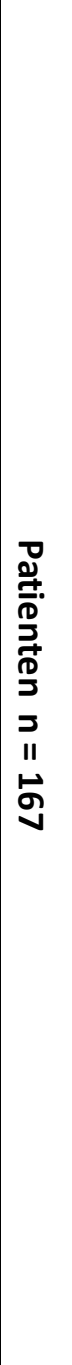 } \\
\hline & ypUICC-I (ypT1/2N0) & TRG 3 & 23 & & \\
\hline & ypUICC-Ila (ypT3N0) & TRG 3 & 18 & & \\
\hline & ypUICC-IIla (ypTON1) & TRG 4 & 2 & & \\
\hline \multirow{7}{*}{ moderat } & \multirow{2}{*}{ ypUICC-I (ypT1/2N0) } & TRG 1 & 3 & \multirow{7}{*}{42} & \\
\hline & & TRG 2 & 12 & & \\
\hline & \multirow{2}{*}{ ypUICC-Ila (ypT3No) } & TRG 1 & 3 & & \\
\hline & & TRG 2 & 20 & & \\
\hline & \multirow{2}{*}{ ypUICC-Ilb (ypT4aN0) } & TRG 2 & 2 & & \\
\hline & & TRG 3 & 1 & & \\
\hline & ypUICC-IIc (ypT4bN0) & TRG 3 & 1 & & \\
\hline \multirow{7}{*}{ schlecht } & \multirow{2}{*}{ ypUICC-IIla (ypT1/2N1, ypT1N2a) } & TRG 2 & 2 & \multirow{7}{*}{42} & \\
\hline & & TRG 3 & 5 & & \\
\hline & & TRG 1 & 3 & & \\
\hline & ypUICC-IIlb (урT1/2 N2b, урT2/3 N2a, ypT3/T4aN1) & TRG 2 & 15 & & \\
\hline & & TRG 3 & 5 & & \\
\hline & \multirow{2}{*}{ ypUICC-IIlc (урT4b N1/2, ypT3/4b N2b, ypT4aN2a) } & TRG 2 & 9 & & \\
\hline & & TRG 3 & 3 & & \\
\hline \multirow{6}{*}{$\begin{array}{c}\text { keine } \\
\text { Änderung }\end{array}$} & \multirow{2}{*}{ ypUICC IVa (jedes Tund jedes N M1a) } & TRG 2 & 5 & \multirow{6}{*}{15} & \\
\hline & & TRG 3 & 5 & & \\
\hline & \multirow{4}{*}{ ypUICC IVb (jedes T und jedes N M1b) } & TRG 1 & 1 & & \\
\hline & & TRG 2 & 2 & & \\
\hline & & TRG 3 & 1 & & \\
\hline & & TRG 4 & 1 & & \\
\hline
\end{tabular}

Tabelle 38: Ansprechen gruppiert nach ypUICC- und sekundär nach TRG-Status *: Ansprechen des Rektumkarzinoms mit cUICC-II oder -III Status auf die präoperative MMT mit Gewichtung des ypUICC-Stadiums und der Tumorregression (TRG 0 bis TRG 4) 
Insofern scheint neben der CRT-induzierten Tumorregression auch der ypUICCStatus eine erhebliche prognostische Bedeutung zu haben. Unter Gewichtung des nach den Vorgaben der TNM-/UICC-Klassifikation erhobenen ypUICC-Status und der Tumorregression (TRG 0 bis TRG 4) wurde das Ansprechen der Rektumkarzinome auf die MMT unabhängig von der Patientenkohorte in ein gutes, moderates und schlechtes Tumoransprechen neu eingeteilt (Tabelle 38).

Nach Ausschluss der ypUICC-IV Stadien ( $n=15$, Tabelle 38) verblieben 152 Patienten, bei denen die Wirksamkeit der Behandlungsmaßnahmen nach dem Astreated-Prinzip untersucht wurde (Tabelle 39).

\begin{tabular}{|c|c|c|c|c|c|c|c|c|c|}
\hline & \multirow{3}{*}{\begin{tabular}{|l} 
TU-Ansprechen \\
gut
\end{tabular}} & & \multirow{3}{*}{$\begin{array}{r}\text { Ereignis } \\
19\end{array}$} & \multicolumn{5}{|c|}{ Überleben (in Monaten) } & \\
\hline & & & & \multirow{2}{*}{$\frac{\text { Median }}{140}$} & \multirow{2}{*}{$\frac{\mathrm{rMW}^{*}}{115,01}$} & \multirow{2}{*}{$\frac{\text { SD }}{6,10}$} & \multicolumn{2}{|c|}{$95 \% \mathrm{Cl} * *$} & logrank \\
\hline \multirow{4}{*}{ DFS } & & & & & & & 103,07 & 126,95 & \multirow{4}{*}{$p=0,002$} \\
\hline & moderat & 42 & 21 & 90 & 84,43 & 8,71 & 67,36 & 101,49 & \\
\hline & schlecht & 42 & 25 & 73 & 78,19 & 9,37 & 59,82 & 96,56 & \\
\hline & $\Sigma^{\# \#}$ & 152 & 65 & 136 & 97,84 & 4,83 & 88,38 & 107,30 & \\
\hline \multirow{4}{*}{ RFS } & gut & 68 & 18 & $\mathrm{nr}^{\star * *}$ & 116,88 & 6,15 & 104,82 & 128,94 & \multirow{4}{*}{$p=0,001$} \\
\hline & moderat & 42 & 20 & 107 & 86,48 & 8,78 & 69,26 & 103,69 & \\
\hline & schlecht & 42 & 25 & 73 & 78,20 & 9,37 & 59,82 & 96,56 & \\
\hline & $\Sigma^{\# \#}$ & 152 & 63 & 136 & 99,08 & 4,26 & 89,59 & 108,57 & \\
\hline \multirow{4}{*}{ CSS } & gut & 68 & 7 & $\mathrm{nr}^{\star * *}$ & 134,67 & 4,44 & 125,97 & 143,37 & \multirow{4}{*}{$p=0,015$} \\
\hline & moderat & 42 & 6 & $\mathrm{nr}^{\star \star \star}$ & 125,49 & 6,90 & 111,96 & 139,01 & \\
\hline & schlecht & 42 & 13 & $\mathrm{nr}^{* * *}$ & 110,60 & 8,64 & 93,66 & 127,54 & \\
\hline & $\Sigma^{\# \#}$ & 152 & 26 & $\mathrm{nr}^{\star \star *}$ & 127,46 & 3,82 & 119,97 & 134,94 & \\
\hline \multirow{4}{*}{ os } & gut & 68 & 16 & $\mathrm{nr}^{\star \star *}$ & 120,83 & 5,77 & 109,52 & 132,13 & \multirow{4}{*}{$p=0,029$} \\
\hline & moderat & 42 & 16 & 132 & 104,36 & 7,77 & 89,13 & 119,60 & \\
\hline & schlecht & 42 & 20 & 103 & 96,51 & 8,58 & 79,70 & 113,31 & \\
\hline & $\Sigma^{\# \#}$ & 152 & 52 & 140 & 110,58 & 4,31 & 102,14 & 119,02 & \\
\hline
\end{tabular}

Tabelle 39: Überleben nach modifizierter Einteilung des Tumoransprechens

*rMW: beschränktes erwartetes Überleben (restricted mean of survival) als Mittelwert für das DFS, RFS, CSS oder OS; die Schätzungen sind auf die längste Überlebenszeit begrenzt, wenn sie zensiert wurden; ${ }^{* *} 95 \%-\mathrm{Cl}$ : 95\%-Konfidenzintervall; ${ }^{* *} \mathrm{nr}$ : not reached, der Medianwert wurde nicht erreicht; "logrank: logrank- Test; ${ }^{\#} \Sigma: n=152$ Patienten ohne ypUICC-IV Stadien 


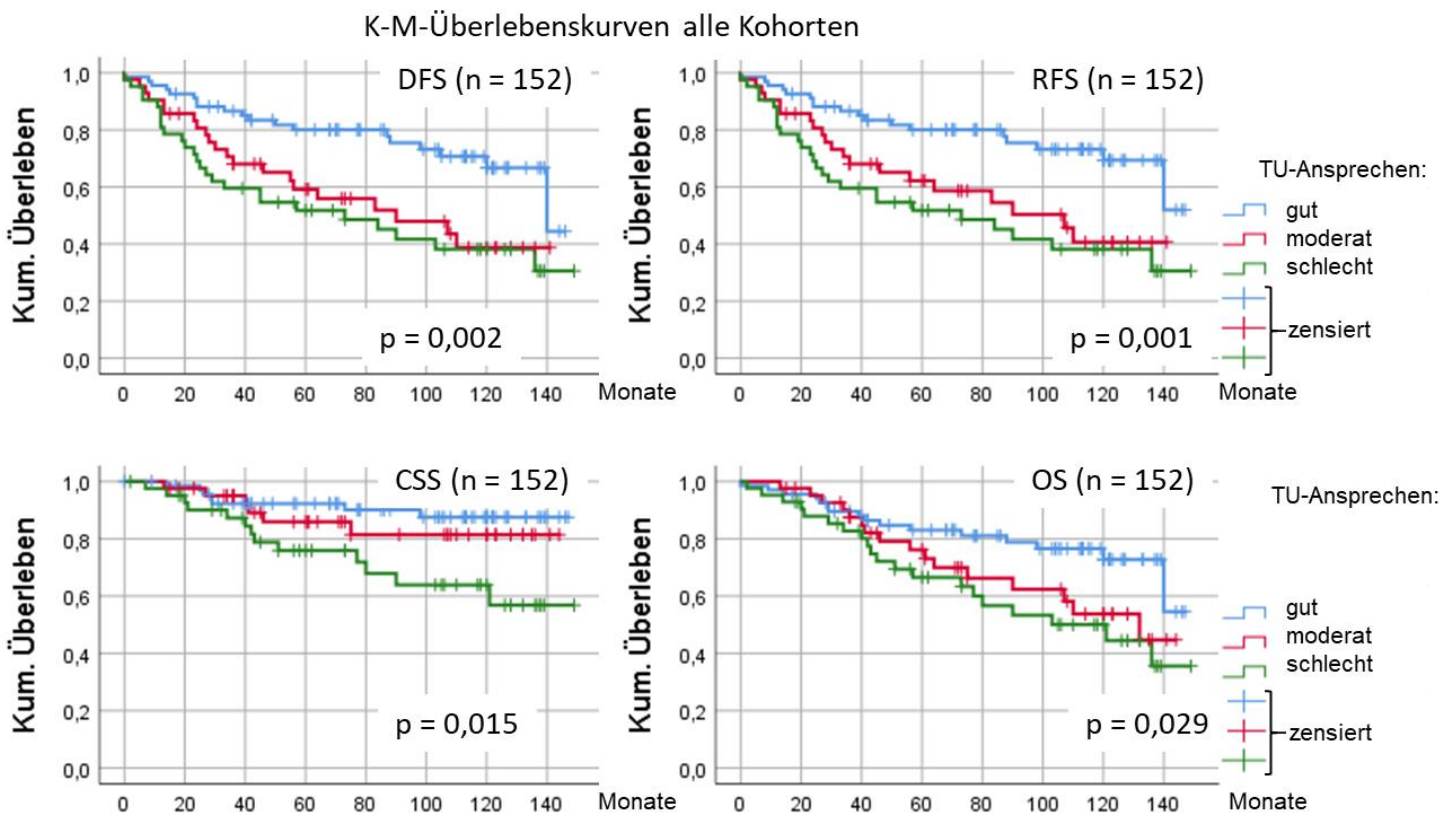

Abbildung 25: K-M-Überlebenskurven (I) nach Ansprechen auf die MMT

Dargestellt sind $n=152$ Patienten einschließlich der postoperativen Therapiewechsler (As-treated-Prinzip); ausgeschlossen wurden Patienten mit residuellen ypUICC-IV Stadien.

K-M-Überlebenskurven alle Kohorten
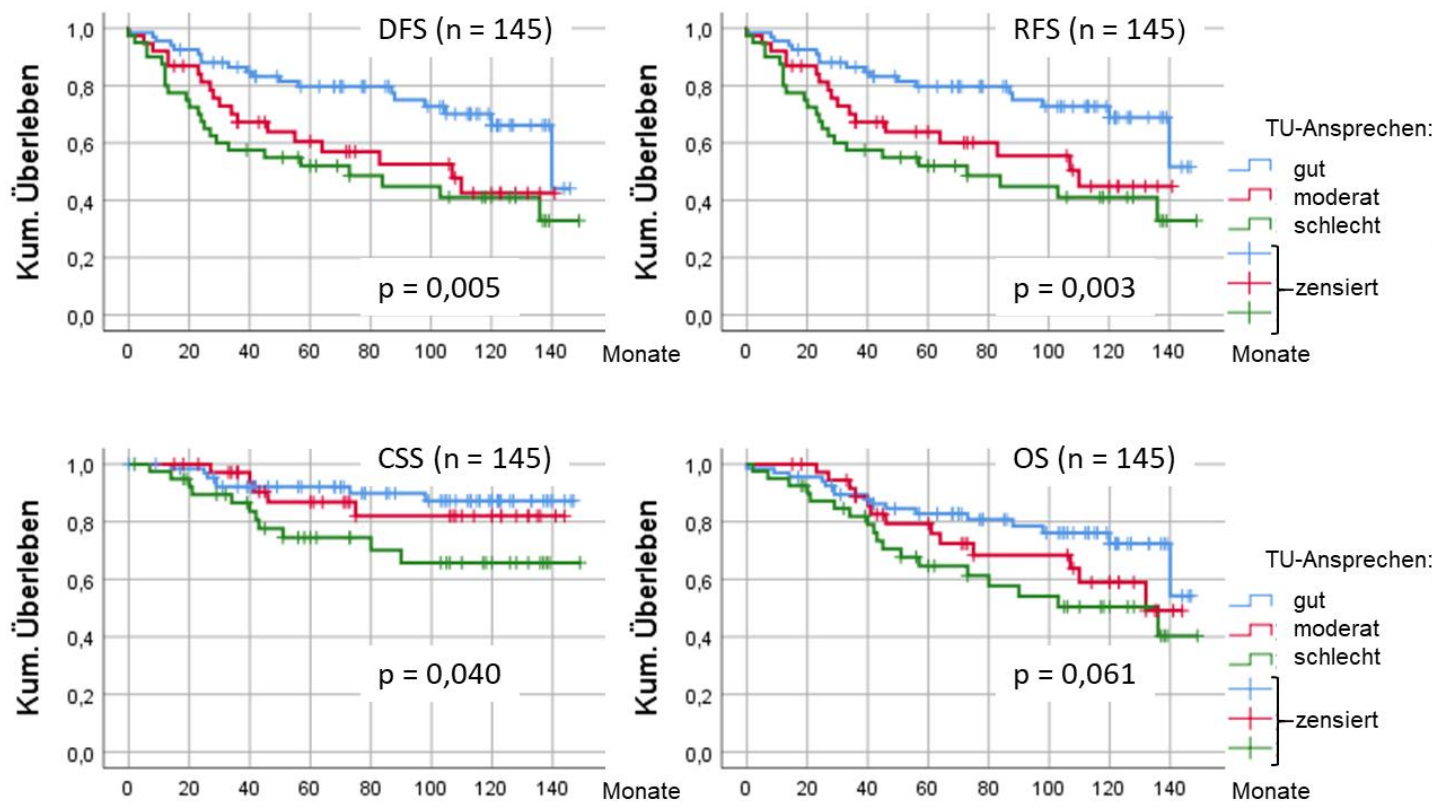

Abbildung 26: K-M-Überlebenskurven (II) nach Ansprechen auf die MMT Kaplan-Meier-Überlebenskurven von $n=145$ Patienten stellen das Tumoransprechen auf die MMT ohne postoperative Therapiewechsler und ohne ypUICC-IV Stadien dar. 
Unter Ausschluss der postoperativen Therapiewechsler und der ypUICC-IV Stadien zeigte sich bei den verbliebenen 145 Patienten ein sehr ähnliches Bild.

\subsubsection{Tumoransprechen - Subgruppen (as-treated)}

In den nachfolgenden Abbildungen ist das Ansprechen auf die perioperative Multimodaltherapie unter Ausschluss der Therapiewechsler und der residuellen ypUICC-IV Stadien Kohorten-spezifisch (as-treated) angegeben. Dargestellt werden die K-M-Überlebenskurven für das RFS und CSS (Abbildung 27) sowie für das DFS und OS (Abbildung 28). Im Beobachtungszeitraum der Kohorte $\mathrm{C}$ mit maximal 86 Monaten weisen die Patienten mit einem guten Tumoransprechen in den K-M-Überlebenskurven höhere Überlebenswahrscheinlichkeiten auf.
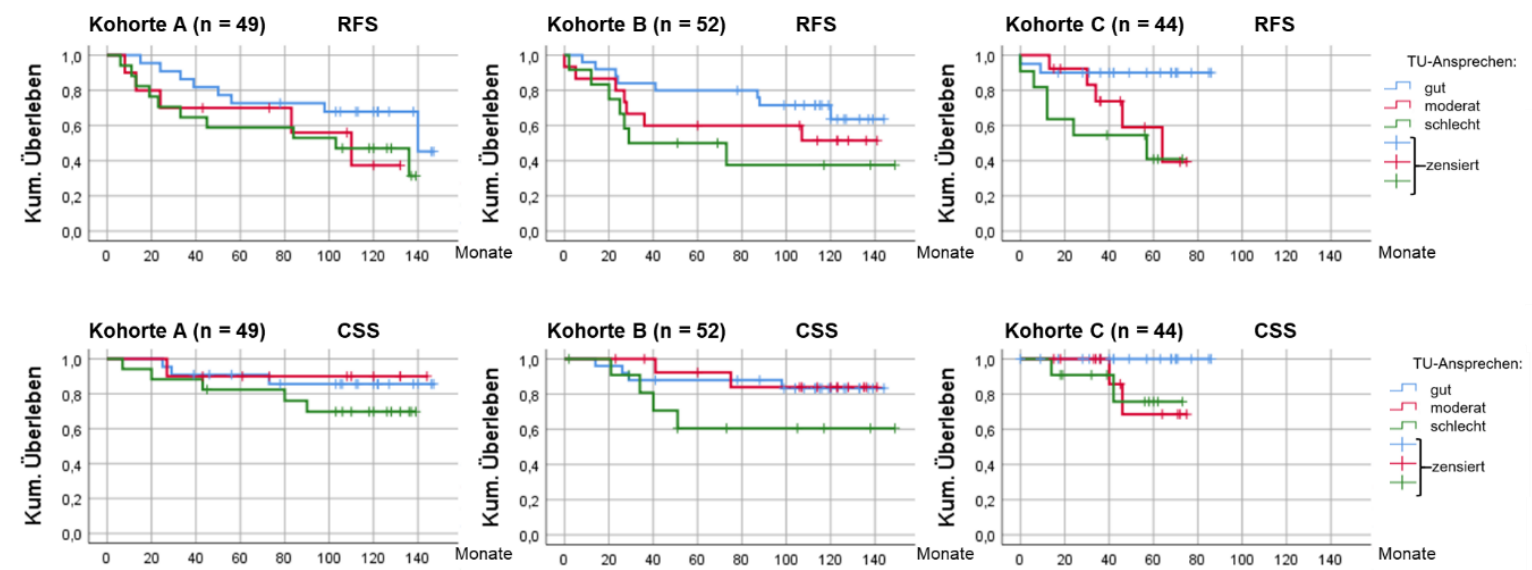

Abbildung 27: RFS und CSS nach Tumoransprechen auf die MMT

Die beobachtete Patientenpopulation $(n=145)$ setzt sich aus 49 Patienten der Kohorte A (adCTx mit 5-FU), 52 Patienten der Kohorte B (adCTx mit FOLFOX) und 44 Patienten der Kohorte C zusammen. Die Darstellung schließt Therapiewechsler (nach erfolgter TME) und Fälle mit ypUICC-IV Stadien aus (As-treated-Prinzip). 

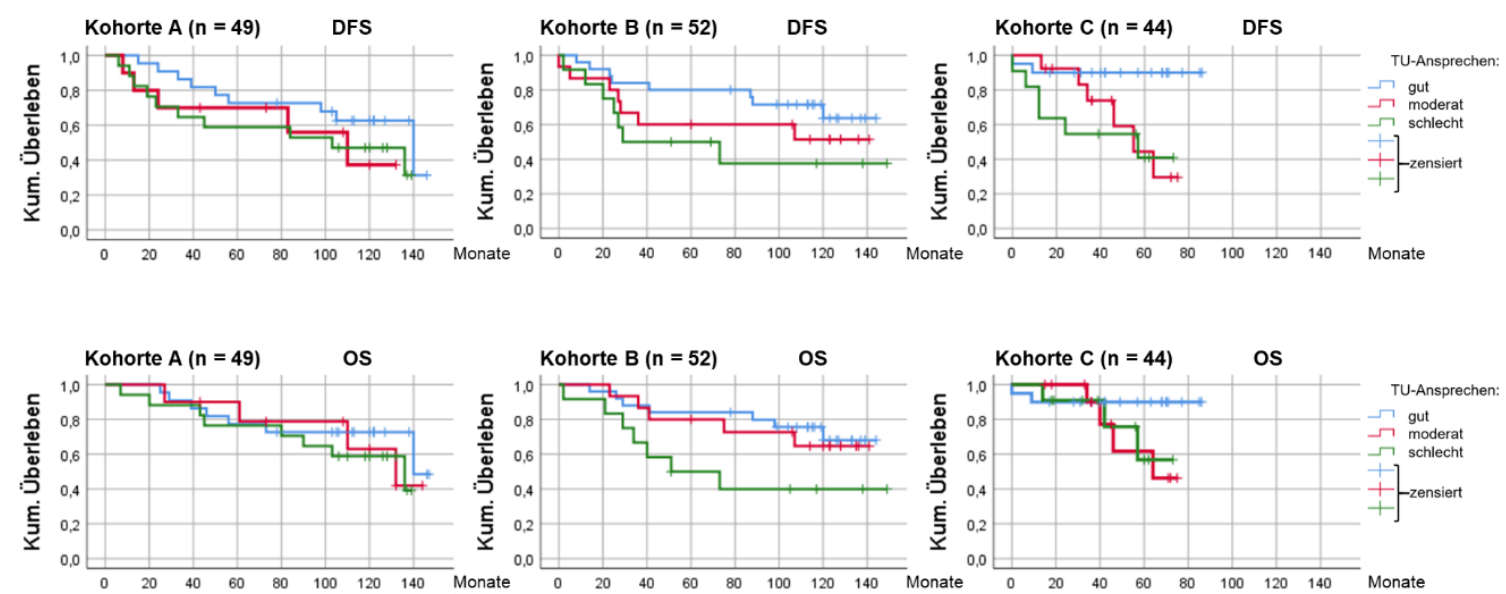

Abbildung 28: DFS und OS nach Tumoransprechen auf die MMT

Die beobachtete Patientenpopulation $(n=145)$ setzt sich aus 49 Patienten der Kohorte $A$ (adCTx mit 5-FU), 52 Patienten der Kohorte B (adCTx mit FOLFOX) und 44 Patienten der Kohorte $C$ zusammen. Die Darstellung schließt Therapiewechsler (nach erfolgter TME) und Fälle mit ypUICC-IV Stadien aus (As-treated-Prinzip).

\subsection{Multivariate Analysen - Überleben - Einfluss von Oxaliplatin}

Zur Klärung des Einflusses von OX in der perioperativen MMT von LARC wurde ein multiparametrisches Überlebenszeitmodell zur Bestimmung des CSS entwickelt. Dies erfolgte auf der Grundlage der Ergebnisse aus den univariaten Analysen (logrank-Test: $\mathrm{p}<0,05)$ und nach manueller Auswahl von Parametern nach klinischer Gewichtung (z. B. Tumoransprechen mit GR vs. PR, p =0,061). Die Variablen umfassten die Kohortenzugehörigkeit (Patientengruppen $A$ bis $C$ ), das Tumoransprechen (GR vs. PR), die residuelle Tumorlängsausdehnung (ypTE $\leq 25 \mathrm{~mm}$ ), den ypL-/V-/Pn-Status (positiv vs. negativ) und den ypUICC-Status. Der Parameter ypCRM wurde aufgrund der geringen Fall- und Ereigniszahl für Patienten mit ypCRM $<2 \mathrm{~mm}$ (Tabelle 37) nicht berücksichtigt. Im Forest-Plot (Abbildung 29) werden die ausgewählten Variablen, die Patienten- und Ereigniszahlen, die Hazard Ratio, die 95\%-Cl- und p-Werte aus dem Cox-Modell angegeben. 


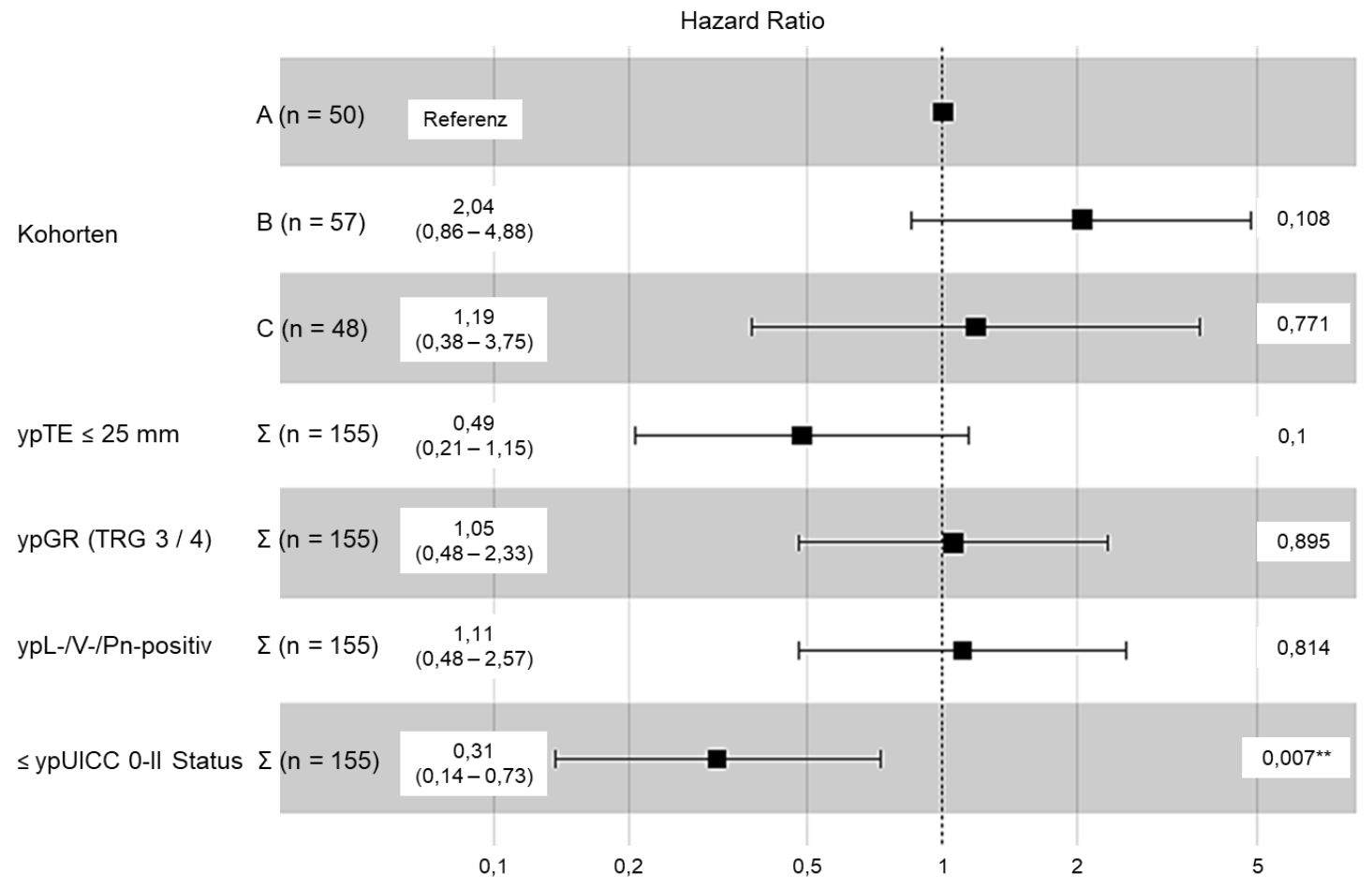

Ereignisse: $\mathrm{n}=28$; globaler $\mathrm{p}$-Wert (logrank): 0,0180; AIC: 262,21; Concordance Index: 0,71

Abbildung 29: Multivariate Analyse (Forest-Plot-Darstellung) zum CSS

Die Patientenkohorte A gilt als Referenzgruppe; das CSS-Modell ist nach manueller Auswahl von Einflussfaktoren (linke Spalte) aus den univariaten Analysen (Kohorten A-C, $n=155, p<0,05$ ) und unter Berücksichtigung klinischer Relevanz (GR vs. PR; $p<0,061$ ) angepasst worden. HR: das Hazard bezeichnet das instantane (augenblickliche) Risiko eines Ereignisses, also die Wahrscheinlichkeit für ein Ereignis im nächsten Zeitschritt, wenn das Ereignis noch nicht eingetreten ist. Die Hazard Ratio (HR) ist das Verhältnis der Hazards in den Kohorten. HR = 1: kein Unterschied zwischen den beiden Patientenkohorten; HR > 1: die Patienten, die mit OX behandelt wurden, haben im Beobachtungszeitraum ein höheres Hazard (instantanes Risiko) als die Referenzgruppe; $\mathrm{HR}<1$ : das Ereignisrisiko für die beobachteten Kohorten B und C (perioperative CTx mit OX) ist kleiner als das Risiko für die Referenzgruppe; 95\%-Cl: 95\%-Konfidenzintervall für die HR; **das vermehrte UICC-Downstaging zu $\leq \mathrm{ypUICC}$-II Stadien ist mit einem verlängerten CSS assoziiert ( $p=0,007$; HR: 0,31; 95\%-Cl: 0,14-0,73).

In der Cox-Regression wurden alle Patienten $(n=155)$ berücksichtigt, die ihre präoperative MMT sowie TME erhalten und - wie geplant - die postoperative Therapie angetreten hatten. Demzufolge sind in Kohorte A (Referenzgruppe) 50 Patienten, in Kohorte B 57 Patienten und in Kohorte C 48 Patienten ausgewertet worden. In der Kohorte $\mathrm{C}$ wurde Patient $\mathrm{H} 123$ aufgrund einer postoperativen R2b-Situation aus diesen Analysen ausgeschlossen. Ebenso wurden die Thera- 
piewechsler ( $n=9$ von Kohorte $A$ nach $B ; n=2$ von Kohorte $B$ nach $A$ ) nicht berücksichtigt.

Im Forest-Plot zeigt sich, dass für die Patienten der Kohorte $C$ ein annähernd gleiches instantanes Risiko besteht wie für die Patienten der Kohorte A (logrank-Test: $\mathrm{p}=0,771$; HR: 1,19; 95\%-Cl: 0,38-3,75). Demgegenüber ist das instantane Risiko für ein Tumorrezidiv in der Kohorte B deutlich, aber nicht signifikant erhöht (logrank-Test: $p=0,108$; HR: 2,04; 95\%-Cl: 0,86-4,88). Patienten mit einer residuellen Tumorausdehnung $\leq 25 \mathrm{~mm}$ profitieren im CSS (logrank-Test: $p=0,1$; HR: 0,49; 95\%-Cl: 0,21-1,15). Im Gegensatz zu den univariaten Analysen zeigt sich kein Einfluss des Tumoransprechens (GR; HR: 1,05; 95\%-Cl: 0,48-2,33) oder des positiven ypL-/V-/Pn-Status (HR: 1,11; 95\%-Cl: 0,48-2,57). Hochsignifikant ist das vermehrte UICC-Downstaging zu $\leq$ ypUICC-II Stadien (logrank-Test: $p=0,007$; HR: 0,31; 95\%-CI: 0,14-0,73) mit einem verlängerten CSS assoziiert. Anhand des Überlebenszeit-Risiko-Modells werden in Abbildung 30 die signifikant unterschiedlichen K-M-Überlebenskurven für das CSS (logrank-Test: $p=0,0002$ ) in den drei Patientenkohorten $\mathrm{A}$ bis $\mathrm{C}$ dargestellt. Dabei werden die Patientenkohorten mit initial geplanter und tatsächlich applizierter adCTx aufgeführt. Insbesondere die Patienten der Kohorten $\mathrm{A}$ und $\mathrm{C}$ zeigen gute CSS-Langzeitverläufe mit einer > 85 \%-igen Wahrscheinlichkeit, ein CSS von > 60 Monaten post operationem zu erreichen. 


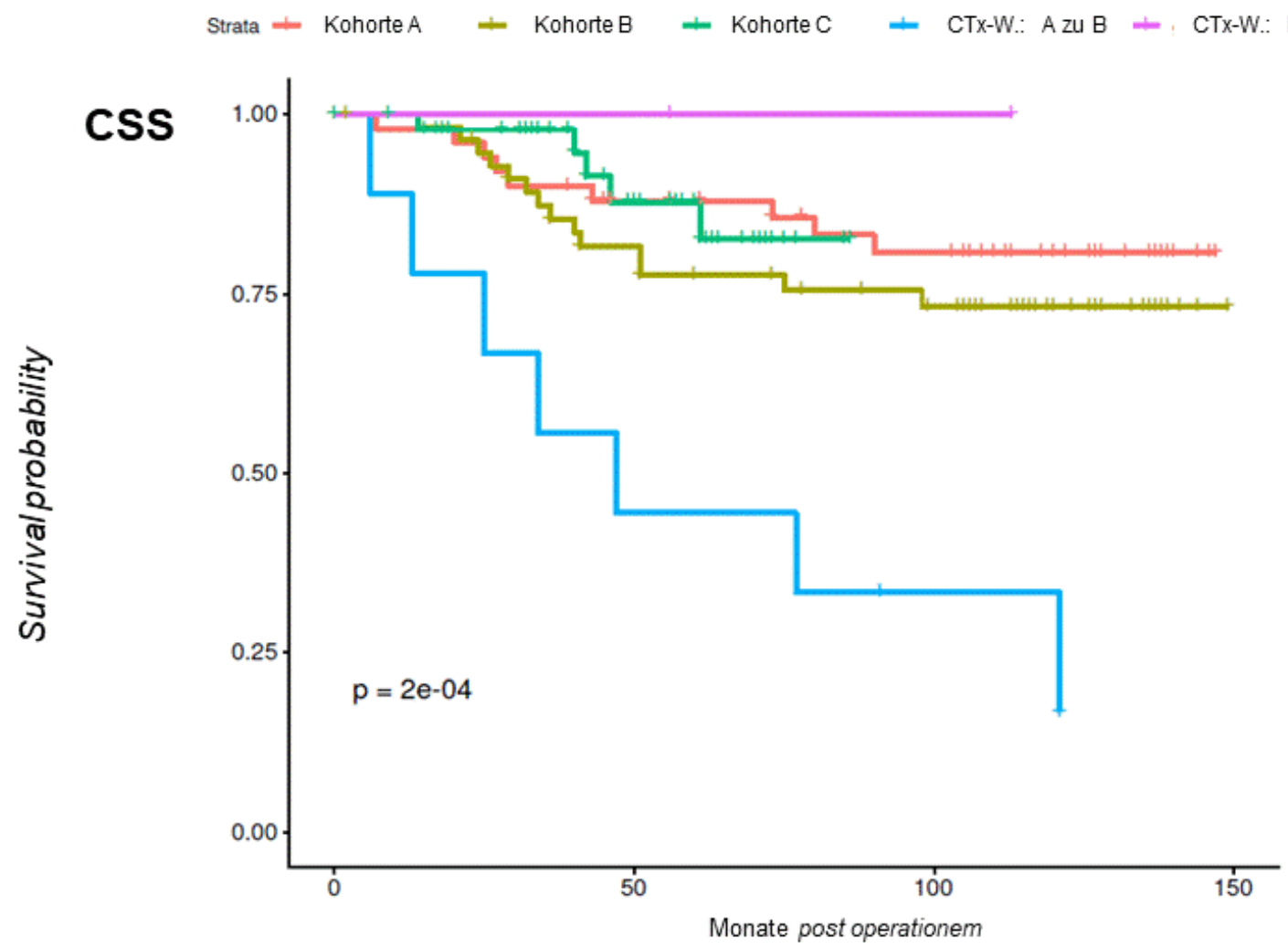

Kohorte A

Kohorte B

Kohorte C

CTx-Wechsler A zu B

CTx-Wechsler B zu A

\section{Number at risk}

$\left\{\begin{array}{cccc}50 & 40 & 33 & 0 \\ 57 & 41 & 31 & 0 \\ 48 & 23 & 0 & 0 \\ 9 & 4 & 2 & 0 \\ 2 & 2 & 1 & 0 \\ \hline 0 & 50 & 100 & 150\end{array}\right.$

Abbildung 30: CSS aller Patienten mit abgeschlossener perioperative MMT und Eintritt in die Nachsorgephase

Patienten $(n=166)$; Kohorte A: $n=50$, Referenzgruppe; Kohorte B: $n=57$; Kohorte C: $\mathrm{n}=48$ Patienten; CTx-W.: postoperative Therapiewechsler, d. h. neun Patienten von Kohorte A erhielten eine FOLFOX-CTx (nach Kohorte B) und zwei Patienten von Kohorte B erhielten eine 5-FU Monotherapie (nach Kohorte A); logrank-Test: $p=0,0002$

Unter den postoperativen Therapiewechslern zeigten die beiden Patienten der ehemaligen Kohorte B (Patient H63 und H83), die bei einem ypUICC-0 und ypUICC-I Status zur adCTx mit 5-FU (Kohorte A) wechselten, einen exzellenten Krankheitsverlauf ohne Hinweis auf ein Tumorrezidiv. Demgegenüber hatten die neun Therapiewechsler aus der ehemaligen Kohorte $A$ trotz der postoperativen FOLFOX-Therapie (nach Kohorte B) einen deutlich schlechteren Verlauf im CSS. 
Diese Patienten scheinen überhaupt nicht von der Hinzunahme von OX zur adCTx profitiert zu haben.

Es handelte sich dabei um neun männliche Patienten, die nach einer kompletten, 5-FU-basierten CRT ohne jegliche Dosisreduktion (Tabelle 40) und nach TME die Stadien ypUICC-I $(n=2)$, ypUICC-IIlla und -IIIc $(n=2)$, ypUICC-IVa $(n=3)$ sowie ypUICC-IVb $(n=2)$ aufwiesen. Eine PR lag in sieben Fällen mit einem Tumorstadium > ypUICC-II vor. Insbesondere Patienten mit einem positiven ypL-/V-/PnStatus schienen nicht von einer adCTx mit FOLFOX zu profitieren und verstarben nach weniger als 48 Monaten post operationem. Die Therapie-Compliance der Therapiewechsler entsprach der hohen Compliance der restlichen Studienpopulation.

\begin{tabular}{|c|c|c|c|c|c|c|c|c|c|c|c|c|}
\hline \multirow{2}{*}{$\begin{array}{c}\text { Patient, Alter, } \\
\text { Gender }\end{array}$} & \multicolumn{3}{|c|}{$\begin{array}{c}\text { Applikationen } \\
\text { von } \mathrm{RT} \text { und } \mathrm{CTx}\end{array}$} & \multirow{2}{*}{$\begin{array}{c}\text { TU-Status } \\
\text { (ypUICC) }\end{array}$} & \multirow{2}{*}{ TRG } & \multirow{2}{*}{$\begin{array}{c}\text { TE } \\
(\mathrm{mm})\end{array}$} & \multirow{2}{*}{$\begin{array}{c}\text { L-/V-/Pn- } \\
\text { positiv }\end{array}$} & \multirow{2}{*}{$\mathrm{R}$} & \multirow{2}{*}{ CRM } & \multirow{2}{*}{ FM } & \multirow{2}{*}{$\begin{array}{l}\text { CSS } \\
(\mathrm{m})\end{array}$} & \multirow{2}{*}{ Tod } \\
\hline & $\begin{array}{c}\text { präop. } \\
\text { RT }\end{array}$ & präop. & $\begin{array}{l}\text { postop. } \\
\text { CTx }\end{array}$ & & & & & & & & & \\
\hline $\mathrm{H} 20,53, \mathrm{~m}$ & $\mathrm{x}$ & $x$ & $\mathrm{x}$ & $3 a$ & 2 & 25 & 0 & 0 & 0 & 1 & 77 & TU \\
\hline $\mathrm{H} 30,63, w$ & $x$ & $x$ & $x$ & $3 c$ & 2 & 10 & 0 & 0 & 0 & 1 & 121 & TU \\
\hline $\mathrm{H} 35,59, \mathrm{~m}$ & $x$ & $x$ & $x$ & 1 & 1 & 25 & 0 & 0 & 0 & 0 & 90 & NTU \\
\hline $\mathrm{H} 41,71, \mathrm{~m}$ & $x$ & $x$ & $x$ & $4 a$ & 3 & 10 & 0 & 0 & 0 & 1 & 121 & lebt \\
\hline $\mathrm{H} 50,55, \mathrm{~m}$ & $x$ & $x$ & $x$ & $4 a$ & 2 & 50 & 0 & 0 & 0 & 1 & 25 & TU \\
\hline $\mathrm{H} 71,62, \mathrm{~m}$ & $x$ & $x$ & $x$ & $4 a$ & 2 & 35 & 1 & 0 & 0 & 1 & 47 & TU \\
\hline $\mathrm{H} 79,64, \mathrm{~m}$ & $x$ & $x$ & $(x)^{*}$ & 1 & 2 & 30 & 1 & 1 & 1 & 1 & 13 & TU \\
\hline $\mathrm{H} 88,65, \mathrm{~m}$ & $x$ & $x$ & $(\mathrm{x})^{* *}$ & $4 \mathrm{~b}$ & 3 & 35 & 1 & 0 & 0 & 1 & 6 & TU \\
\hline $\mathrm{H} 168,54, \mathrm{~m}$ & $x$ & $x$ & $x$ & $4 \mathrm{~b}$ & 1 & 70 & 1 & 2 & 1 & 1 & 34 & TU \\
\hline
\end{tabular}

Tabelle 40: Daten der Therapiewechsler, die FOLFOX als adCTx erhielten

$\mathrm{X}=$ vollständige Applikationen; $\mathrm{x}=$ inkomplette Applikationen ${ }^{*}(\mathrm{x})$ : bei diesem Patienten waren der zweite und dritte Zyklus der adCTx mit FOLFOX unter OX-Dosisreduktion auf $75 \%$ erfolgt; kein OX im vierten Zyklus, Abbruch der adCTx vor der achten Applikation; ${ }^{* *}(\mathrm{x})$ : der Patient hatte im zweiten Zyklus der adCTx eine Reduktion von FOLFOX auf $75 \%$ erhalten, im dritten Zyklus FOLFOX auf $50 \%$ und im vierten Zyklus FOLFOX auf $25 \%$; vor der achten Applikation erfolgte dann der Abbruch der adCTx; TRG: Tumorregressionsgrad (1 und 2 partial response, 3 good response); TE: Tumorausdehnung; R: Residualstatus; CRM: zirkumferentieller Resektionsrand $(0: \geq 2 \mathrm{~mm}, 1:<2 \mathrm{~mm})$; FM: Nachweis von Fernmetastasen ( $1=$ Nachweis, $0=$ kein Nachweis); CSS: krebsspezifisches Überleben in Monaten; TU: tumorbedingter Tod; NTU: nicht tumorbedingter Tod; der Patient verstarb aufgrund einer kardialen Ursache.

Der auch unter FOLFOX-CTx nicht zu beeinflussende Krankheitsverlauf der Therapiewechsler zeigt sich ebenso im ypUICC-Stadien-adaptierten Vergleich der 
K-M-Überlebenskurven (Abbildung 31). Im CSS haben die Patienten aus Kohorte A und $C$ mit ypUICC-0 und ypUICC-I Status exzellente Langzeitverläufe. Es scheinen zwischen den Patienten dieser beiden Kohorten keine relevanten Überlebenszeitunterschiede für die Tumorstadien ypUICC-0 bis -III zu bestehen.

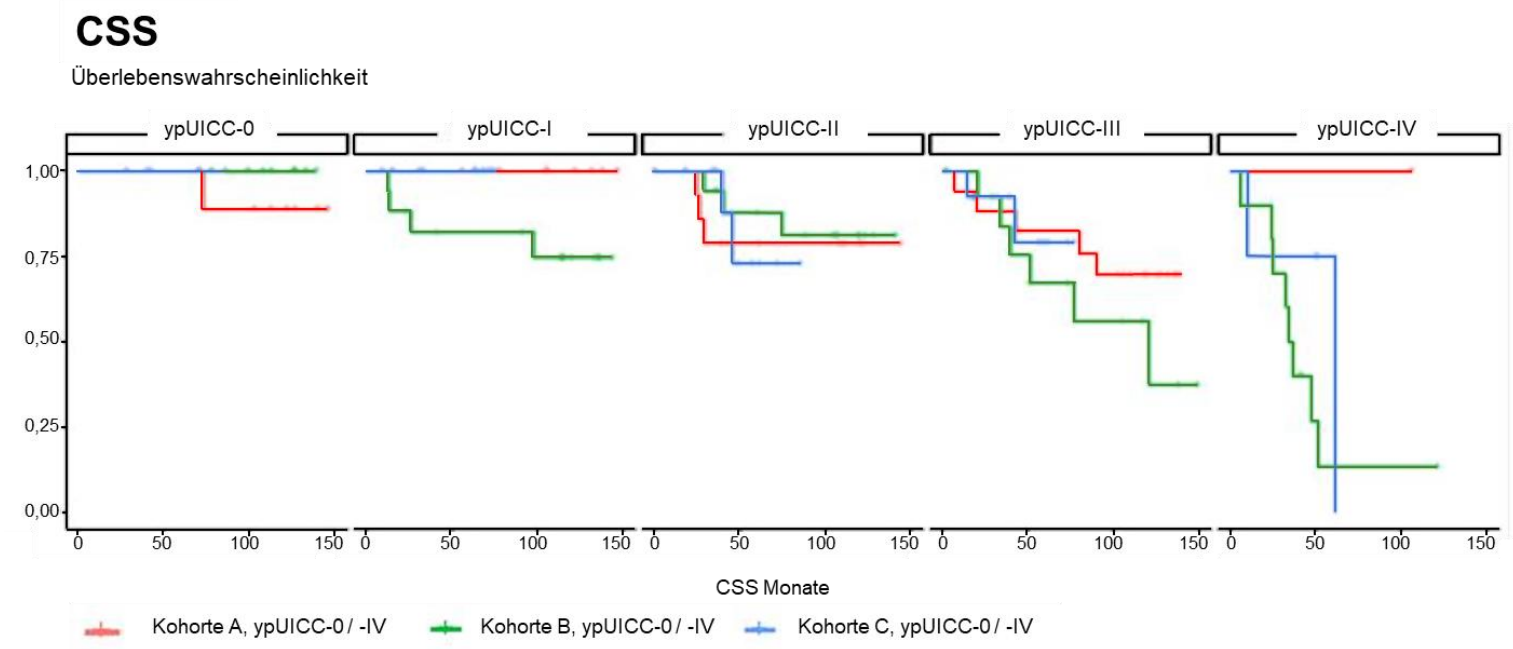

Abbildung 31: CSS in Korrelation zu den ypUICC-Stadien und Patienten-Kohorten Die Darstellung berücksichtigt 155 Patienten ohne die postoperativen Therapiewechsler; Kohorte A: $n=50$; Kohorte B: $n=57$; Kohorte C: $n=48$ Patienten.

Weiterhin auffällig ist beim Vergleich der K-M-Überlebenskurven zum CSS, dass Patienten der Kohorte B bereits früh im Stadium ypUICC-I ein schlechteres CSS haben als die Patienten der Kohorte A.

Es kann darüber spekuliert werden, ob dieser etwas überraschende Unterschied aus einem reversen Will-Rogers-Phänomen, einer frühzeitig erworbenen Therapieresistenz oder aus einer durch die MMT unbeeinflussbaren, genetisch festgelegten Malignombiologie resultiert (Abschnitt 4.1.4; Anmerkung: Das Will-RogersPhänomen (Stage-migration-Effekt) bezeichnet einen Effekt in der Bildung von Mittelwerten in Gruppen. Durch einen Wechsel eines Gruppenelements von der einen zur anderen Gruppe kann der Mittelwert in beiden Gruppen steigen oder absinken. So kann in einer statistischen Analyse über eine Malignom-bedingte Lebenserwartung allein durch eine verbesserte Diagnostik eine effektivere Therapie vorgetäuscht werden, wie z. B. bei der Früherkennung von (Tumor-) Erkrankungen (Feinstein et al. 1985)). 


\section{Diskussion}

Adenokarzinome des Kolons und des Rektums gehören weltweit zu den drei häufigsten Malignomerkrankungen. Abgesehen von der onkologischen Chirurgie, die im Frühstadium primärer Kolon- und Rektumkarzinome weiterhin die Therapie der Wahl darstellt, ist beim Kolonkarzinom im Stadium pUICC-III eine 5-FU-basierte adCTx Standard. Diese wird auch für sogenannte high-risk Fälle im Stadium pUICC-II empfohlen. Zur systemischen Behandlung wird vorzugsweise eine CTXKombination nach dem FOLFOX-Regime eingesetzt, deren bisher übliche Applikationsdauer über 6 Monate mittlerweile kontrovers diskutiert wird (Grothey et al. 2018, André et al. 2013).

\subsection{Allgemeiner Stellenwert der adCTx beim Rektumkarzinom}

Im Gegensatz zu den Kolonkarzinomen ist der Stellenwert einer adCTx bei fortgeschrittenen Rektumkarzinomen ( $\geq$ UICC-Stadium II) ungeklärt. Eine systematische Cochrane Analyse von 21 klinischen Studien (Zeitraum: 1975-2011; 11 Studien in westlichen Ländern, 10 Studien in Japan) mit 9785 Patienten (Petersen et al. 2012), die entweder zu einer 5-FU-basierten $\operatorname{adCTx}(n=4854)$ oder alleinigen Nachbeobachtung $(n=4367)$ randomisiert worden waren, ergab für die adCTx eine Reduktion des Sterberisikos um $17 \%$ (HR: 0,83; 95\%-Cl: 0,76-0,91), eine Senkung des Rezidiv-Risikos um $25 \%$ (HR: 0,75; 95\%-Cl: 0,68-0,83) und keinen zusätzlichen, signifikanten Einfluss auf das DFS und OS. Nach den o.g. Studien folgerten die Autoren, dass unter bestimmten Voraussetzungen eine 5-FU-basierte adCTx für lokal fortgeschrittene Rektumkarzinome von Vorteil sein könnte. Dabei legten die Autoren aber nicht fest, welche definierten UICC-Stadien von der adCTX profitieren könnten, sondern forderten allgemein zu einer Intensivierung der CTx durch Hinzunahme von OX auf. Außerdem empfahlen sie die Umsetzung der infusionalen 5-FU-CTx auf eine orale 5-FU-CTx mit Capecitabin (Xeloda®) in Analogie zur Therapie von Kolonkarzinomen.

In weiteren randomisierten Studien wurden die Ergebnisse der adCTx bei Rektum- 
karzinom-Patienten nach einer präoperativen RT bzw. CRT und TME geprüft (Breugom et al. 2015a; Bosset et al. 2014; Glynne-Jones et al. 2014; Sainato et al. 2014). In diesen klinischen Studien waren die positiven Auswirkungen einer adCTx auf das OS gering. Eine allgemeingültige Empfehlung zur Gabe der adCTx blieb in der Folge ebenfalls aus. Zudem bezweifelten die Autoren, dass mit einer adCTx nach präoperativer MMT ein ähnlich positiver OS-Effekt erreicht werden könnte wie bei pUICC-III Kolonkarzinomen (Gramont et al. 2000). Als Bestätigung wurden die Langzeitergebnisse ( $>5$ Jahre an Nachsorgezeiten) aus der EORTC22921-Studie gewertet, die im Gegensatz zu früheren Subgruppen-Analysen (Collette et al. 2007) keine Verbesserung des DFS und OS durch die adCTx gezeigt hatten (Bosset et al. 2014).

Die ADORE-Phase-II-Studie prüfte (ClinicalTrials.gov, No.: NCT00807911), inwiefern Patienten mit ypUICC-II oder -III Rektumkarzinomen nach einer Fluoropyrimidin-basierten CRT (RT mit 5-FU oder Capecitabin) und TME von einer adCTx mit vier Zyklen 5-FU + FS (5-FU: $380 \mathrm{mg} / \mathrm{m}^{2}$ und FS: $20 \mathrm{mg} / \mathrm{m}^{2}$ ) oder mit acht Zyklen FOLFOX (OX: $85 \mathrm{mg} / \mathrm{m}^{2}$ und FS: $200 \mathrm{mg} / \mathrm{m}^{2}$ und 5-FU: Bolus $400 \mathrm{mg} / \mathrm{m}^{2}$ an d 1; 5-FU: $2400 \mathrm{mg} / \mathrm{m}^{2}$ c.i.v. über $46 \mathrm{~h}$ ) profitieren könnten (Hong et al. 2014). Der primäre Endpunkt dieser koreanischen Multizenter-Studie war das 3-Jahre DFS (Intention-to-treat-Prinzip), das bei der Fallzahlplanung mit $70 \%$ für die Kontrollgruppe und mit $78 \%$ für die FOLFOX-Gruppe angenommen worden war. Zwischen 11/2008 und 12/2012 konnten 321 Patienten mit einem ypUICC-II oder -III Rektumkarzinom in die Studie aufgenommen und unabhängig von der präoperativen CRT in die adCTx-Arme randomisiert werden. Nach einem medianen Follow-up von 38,2 Monaten (interquartil range (IQR): 26,4-50,6 Monate) betrug das DFS bei 295 auswertbaren Patienten in der FOLFOX-Gruppe 71,6\% (95\%-Cl: 64,678,6\%) und in der Kontrollgruppe (adCTx: 5-FU + FS) 62,9\% (95\%-Cl: 55,470,4 \%). Die HR lag bei 0,657 (95\%-Cl: 0,434-0,994; $p=0,047$; Hong et al. 2014). In der aktualisierten Analyse der ADORE-Phase-II-Studie zeigte sich nach einem medianen Follow-up von 74,1 Monaten (IQR: 56,2-88,0 Monate) für die FOLFOXGruppe ein signifikant besseres 6-Jahre DFS mit 68,2 \% gegenüber 56,8 \% (Kontrollarm mit 5-FU + FS). Die stratifizierte HR betrug 0,63 (95\%-Cl: 0,43-0,93; 
$p=0,018)$ in der Intention-to-treat-Analyse. Die adCTx mit FOLFOX erbrachte jenen high-risk Patienten Vorteile im DFS, die postoperativ ein ypUICC-III Stadium, einen ypN1b-, ypN2-, ypG3-Status, eine geringe Tumorregression und/oder einen negativen ypL-/V-/Pn-Status gehabt hatten. Das 6-Jahre OS betrug 78,1\% in der FOLFOX-Gruppe und $76,4 \%$ in der Kontrolle. Die HR war 0,73 (95\%-Cl: $0,45-1,19 ; p=0,21)$. Auch in den weiteren Subgruppenanalysen zum OS war die adCTx mit FOLFOX vorteilhaft für Patienten mit einem ypN2-Status und minimaler, CRT-induzierter Tumorregression (Hong et al. 2019).

Die Ergebnisse der ADORE-Studie gaben erstmals Hinweise darauf, dass Patienten mit Rektumkarzinomen der Stadien ypUICC-II und -III nach Fluoropyrimidinbasierter präoperativer CRT und TME signifikant von einer adCTx mit FOLFOX im DFS profitieren können. Die ADORE-Studienresultate stehen z. T. im Einklang mit den eigenen Ergebnissen zum DFS, RFS und OS für Patienten der Kohorte B mit den Tumorstadien ypUICC-0 und -II. Abweichend zur ADORE-Studie zeigte sich in den eigenen multivariaten Analysen bei Patienten mit einem ypUICC-III Status nach OX-haltiger MMT kein Überlebensvorteil. Vielmehr muss bei Nachweis von > ypUICC-IIlb Tumorstadien von einem derart ungünstigen Verlauf (Kohorte $B$, Abbildung 29) ausgegangen werden, sodass eine Empfehlung für eine FOLFOX-CTx unzureichend ist. Es sollte für betroffene Patienten eine adCTx mit FOLFIRI oder besser mit FOLFOXIRI +/- moAk angestrebt werden.

Trotz der kleinen Fallzahlen bleibt aus dem multivariaten CSS-Überlebensmodell (Abbildungen 29 und 31) bemerkenswert, dass insbesondere Patienten mit einem residuellen positiven ypL-/V-/Pn-Status von einer erweiterten MMT profitieren können. Gerade diese Patienten sollten durch eine adCTx mit FOLFOXIRI Überlebensvorteile erreichen. Zur Klärung dieser Hypothese bedarf es jedoch der Analyse größerer Patientenpopulationen aus randomisierten klinischen Studien und der Initiierung neuer Studienprojekte. Diese Studien sollten nach einer ersten Randomisation in eine präoperative OX-haltige MMT +/- mFOLFOX (analog Kohorte C) postoperativ erneut einer zweiten Randomisation vor Applikation einer adCTx +/OX zugeführt werden. Bei dieser zweiten Randomisation sollte der postoperativ erhobene ypUICC-Status als Stratifikationsmerkmal eingesetzt werden. 


\subsubsection{Stellenwert einer vollständigen Applikation der MMT}

Die prognostische Bedeutung einer vollständig applizierten adCTx ist kürzlich bei 392 LARC-Patienten nach präoperativer CRT und TME gezeigt worden (SandraPetrescue et al. 2018). Die adCTx bestand aus Capecitabin $(n=197)$ oder infusionalem 5-FU $(n=195)$. Bei $64 \%(n=251)$ der Studienteilnehmer konnte die Zahl der geplanten CTx-Zyklen vollständig verabreicht werden. Das 5-Jahre OS dieser Patienten war signifikant höher gegenüber Patienten mit einer unvollständigen $\operatorname{adCTx}(76,0 \%$ vs. 60,6 \%, p < 0,0001). In der Post-hoc-Analyse hatten 31 Patienten der Studienpopulation $(n=392)$ ein $O S<6$ Monaten. Die dokumentierten Gründe für ein vorzeitiges Studienende waren der Tod (zehn Fälle, davon waren zwei Fälle tumorassoziiert und ein Fall aufgrund schwerer Toxizität), die Rücknahme der Einverständniserklärung (11 Patienten), ein Kontaktabbruch (ein Patient), schwere Protokollabweichungen (drei Patienten), schwere Toxizität (ein Patient) sowie sonstige Gründe (vier Patienten). Bei 361 Patienten, die ein OS von $\geq 6$ Monaten aufwiesen, wurde der Einfluss einer kompletten vs. inkompletten Applikation der adCTx-Zyklen auf das 5-Jahre OS und DFS geprüft. Das 5-Jahre OS war bei 251 Patienten (69,5\%), die alle adCTx-Zyklen erhalten hatten, signifikant verlängert $(76,0 \%$ vs. 66,4 \%, p =0,0073). Auch das 5-Jahre DFS war nach der adCTx tendenziell besser $(64,9 \%$ vs. 58,7\%, $p=0,065)$. Die HR für inkomplette adCTx-Zyklen vs. komplette adCTx-Zyklen betrug 1,42 (95\%-Cl: 0,98-2,07). Zudem zeigte die Cox-Regression ein tendenziell längeres OS $(p=0,061)$ und DFS ( $p=0,083$ ) für Patienten, die $\geq 6$ Monate post operationem überlebt und die adCTx komplett erhalten hatten.

In der eigenen Patientenpopulation betrug das mediane DFS 110 Monate (95\%-Cl: 64,60-155,40 Monate) nach vollständiger Applikation der adCTx. Demgegenüber hatten Patienten mit inkomplett applizierter adCTx ein medianes DFS von 107 Monaten (95\%-Cl: 5,88-208,12 Monate). Das mediane OS lag bei 140 Monaten gegenüber 120 Monaten für Patienten mit inkompletter adCTx. In Tabelle 41 sind die rMWs und Standardabweichungen angegeben. Es zeigt sich kein signifikanter Unterschied im DFS und OS der Patienten mit inkompletter vs. kompletter adCTx. 


\begin{tabular}{|c|c|c|c|c|c|c|c|}
\hline & \multicolumn{7}{|c|}{ Überleben (DFS, in Monaten) } \\
\hline & $\mathrm{N}$ & Ereignisse & $\mathrm{rMW}^{*}$ & SD & \multicolumn{2}{|c|}{$95 \%-\left.\mathrm{C}\right|^{* *}$} & $\log r a n k^{* * *}$ \\
\hline CTx komplett & 75 & 38 & 95,54 & 6,57 & 82,67 & 108,41 & \multirow{3}{*}{$p=0,374$} \\
\hline CTx inkomplett & 43 & 23 & 77,84 & 9,18 & 59,85 & 95,84 & \\
\hline $\boldsymbol{\Sigma}$ & 118 & 61 & 90,69 & 5,56 & 79,8 & 101,59 & \\
\hline & \multicolumn{7}{|c|}{ Überleben (OS, in Monaten) } \\
\hline & $\mathrm{N}$ & Ereignisse & rMW* & SD & \multicolumn{2}{|c|}{$95 \%-\left.\mathrm{Cl}\right|^{* *}$} & logrank*** \\
\hline CTx komplett & 75 & 31 & 110,54 & 5,69 & 99,38 & 121,7 & \multirow{3}{*}{$p=0,236$} \\
\hline CTx inkomplett & 43 & 23 & 91,69 & 7,78 & 76,44 & 106,94 & \\
\hline $\boldsymbol{\Sigma}$ & 118 & 52 & 105,37 & 4,82 & 95,92 & 114,81 & \\
\hline
\end{tabular}

Tabelle 41: DFS (rMW) und OS (rMW) der Patienten in Abhängigkeit zu Applikationen/Zyklen adCTx

Angegeben sind das krankheitsfreie (DFS) und gesamte Überleben (OS) während des Therapieverlaufs (prä- und postoperativ) für die Kohorten A und B; die Kohorte C wird nicht betrachtet, da keine adCTx vorgesehen war. rMW*: beschränktes erwartetes Überleben (restricted mean of survival) als Mittelwert für das DFS und OS, diese Schätzung ist auf die längste Überlebenszeit begrenzt, wenn sie zensiert wurde; SD: Standardabweichung; 95\%-Cl**: 95\%-Konfidenzintervall; für DFS: Chi-Quadrat 0,789, 1 Freiheitsgrad; logrank $^{* * *}$ : logrank-Test: $p=0,374$; für OS: Chi-Quadrat 1,404, 1 Freiheitsgrad, logrank-Test: $p=0,236$

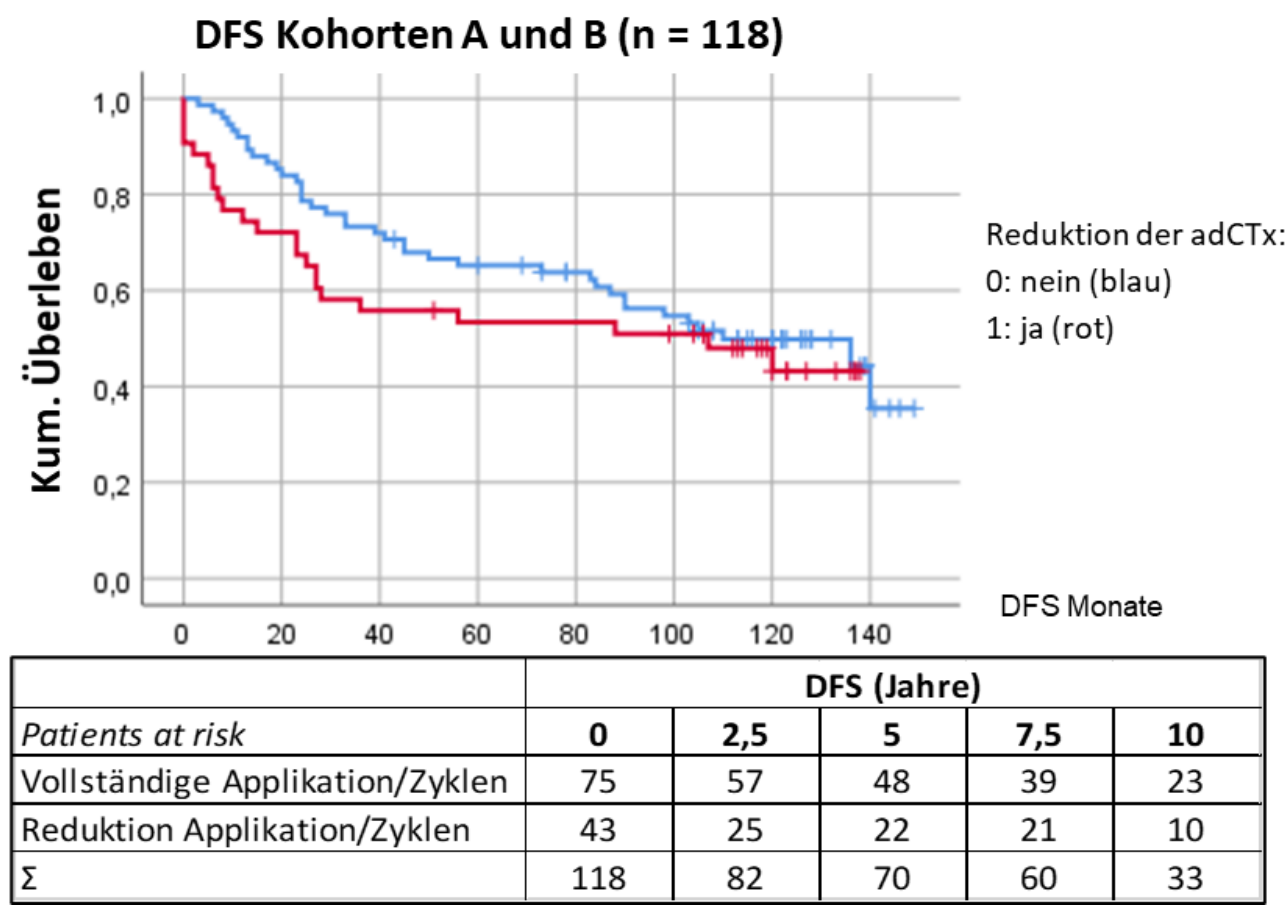

Abbildung 32: DFS in Abhängigkeit zu kompletten Applikationen/Zyklen der adCTx 


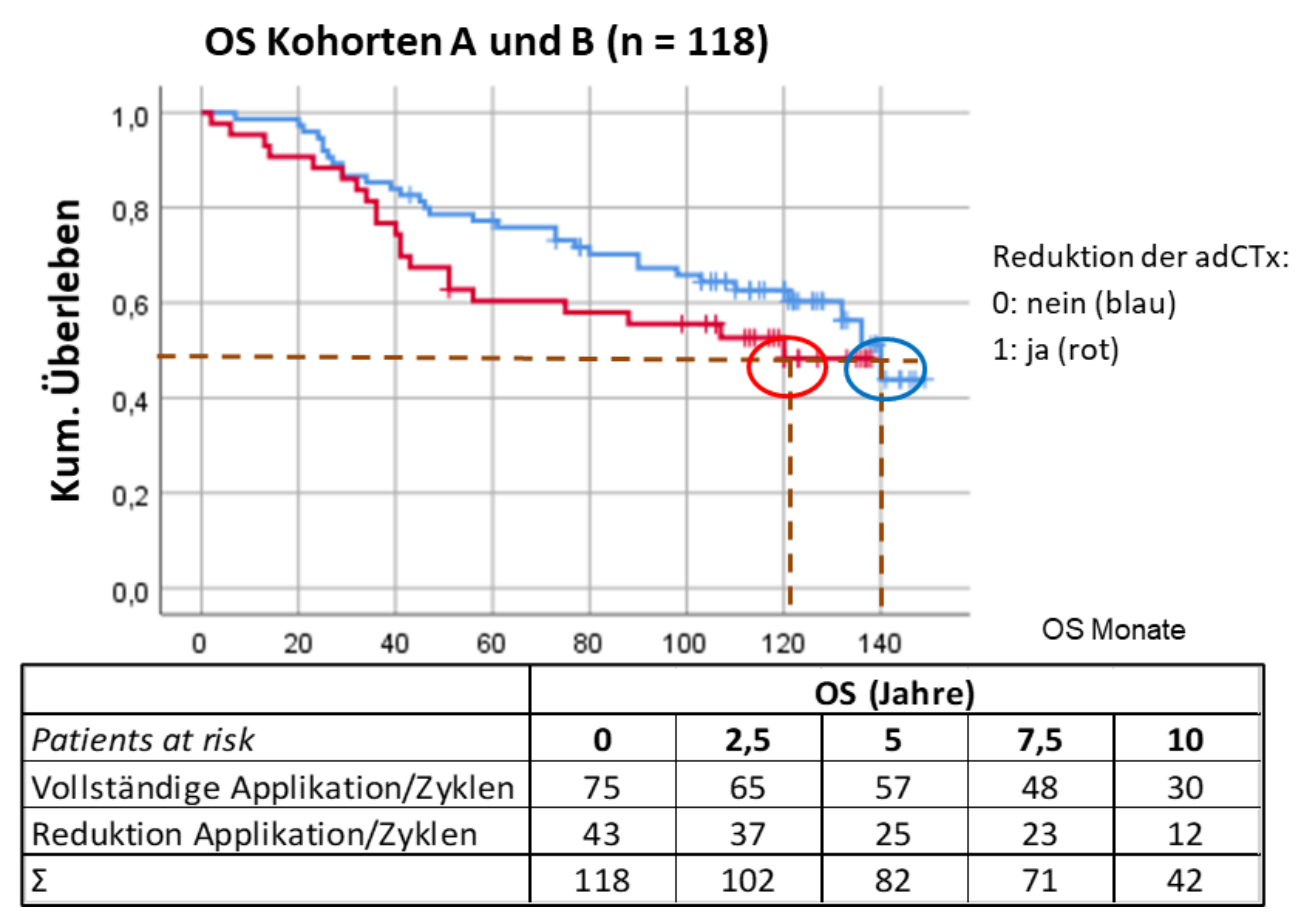

Abbildung 33: OS in Abhängigkeit zu kompletten Applikationen/Zyklen der adCTx

Angegeben sind die Ergebnisse für die Kohorten A und B; blauer Kreis: mediane Überlebenswahrscheinlichkeit der Patienten mit vollständigen Applikationen/Zyklen; roter Kreis: mediane Überlebenswahrscheinlichkeit der Patienten mit inkompletten Applikationen/Zyklen der adCTx.

\subsubsection{Einfluss des postoperativen LK-Status auf die adCTx}

Die Effizienz einer adCTx kann auch von der Anzahl prognostisch ungünstiger pUICC-III Stadien und den untersuchten Lymphknoten abhängen. So wurde z. B. bei insgesamt 939 Patienten mit Kolonkarzinomen, die zwischen 01/2000 und 12/2009 eine onkologische Kolonresektion erhalten hatten, der Einfluss der LKAusbeute bei 142 Patienten mit einem pUICC-III Stadium untersucht (Schiffmann et al. 2013). Dabei wurde das Verhältnis zwischen LKM zu tumorfreien Lymphknoten (LK-Ratio, medianer Cut-off: 0,2 LKM) bestimmt. Anschließend wurde die prognostische Relevanz einer Einteilung in niedrige (LK-Ratio: $<0,2$ ) und hohe Risikogruppen (LK-Ratio: > 0,2) gegenüber der pN1- und pN2-Kategorisierung aus der TNM-/UICC-Klassifikation geprüft. Sowohl die international anerkannte pNKategorisierung als auch die LK-Ratio-Gruppierung zeigten eine starke Korrelation mit dem DFS und CSS. Zwischen den beiden LK-Klassifikationssystemen bestand kein signifikanter Unterschied. 
In den Patientenkohorten A bis C (Tabelle 14) waren unabhängig von der MMT hohe Werte an untersuchten LK und LKM pro Rektumresektat erreicht worden. Ebenso wie die TME-Qualität spiegelt die hohe LK-Ausbeute eine sehr gute Behandlungsqualität im untersuchten Patientenkollektiv wider.

\subsubsection{Einfluss des präoperativen Stagings auf die adCTX}

Ein Grund für die anhaltend kontroverse Beurteilung der adCTx bei Rektumkarzinomen nach präoperativer CRT liegt darin, dass in vielen Studien keine standardisierte Ausbreitungsdiagnostik durchgeführt worden war (Carvalho und Glynne-Jones 2017). Hohe Overstaging-Raten (Bosset et al. 2014; Sainato et al. 2014) in den uneinheitlich eingesetzten Untersuchungsverfahren und eine damit verbundene unzureichende prätherapeutische Einschätzung der Malignomsituation veranlassten viele Kliniker, die Studienergebnisse zur Wirksamkeit einer adCTx anzuzweifeln. So war z. B. weder in der EORTC-Studie (Bosset et al. 2014) noch in der italienischen SNCNR-Studie (Sainato et al. 2014) ein pelvines MRT Voraussetzung für die Studienaufnahme und spätere Randomisation der Patienten. In der Folge konnten Imbalancen nicht ausgeschlossen werden, die den Nutzen einer adCTx möglicherweise verdeckten (Bosset et al. 2014; Sainato et al. 2014).

Interessanterweise weisen aktuellere Studien mit einer postoperativ erfolgten Randomisation, wie die bereits genannte ADORE-Studie, auf Überlebensvorteile durch eine an die pUICC-Stadien angepasste adCTx hin (Hong et al. 2019; Breugom et al. 2015b; Rödel et al. 2015; Glynne-Jones et al. 2014; Hong et al. 2014). Im vorliegenden Projekt bestand das Staging vor Einleitung der MMT aus der rektal-digitalen Untersuchung, einer starren Rektosigmoidoskopie und flexiblen Koloskopie, einer rES, einem CT des Abdomens und Beckens sowie einer pelvinen MRT. In Analogie zum Therapiearm A der CAO/ARO/AIO-94-Studie, bestehend aus einer Rektumresektion, postoperativen CRT und adCTx, muss ohne Einsatz der pelvinen MRT eine potentielle Overstaging-Rate von $18 \%$ angenommen werden (Sauer et al. 2004). Unter systematischer Nutzung der MRT kann von einer deutlich geringeren Overstaging-Rate ausgegangen werden. 


\subsubsection{UICC-Downstaging - offene Fragen}

In klinischen Studien konnte gezeigt werden, dass mit einer präoperativen CRT in bis zu $15 \%$ der Patienten eine komplette Remission (ypCR) des Rektumkarzinoms erreicht werden kann. Zudem konnte bei $>20 \%$ der Patienten durch die Vorbehandlung ein ypUICC-Stadium I erreicht werden (Rödel et al. 2015; Sauer et al. 2012; Maas et al. 2010). In der eigenen Untersuchung betrug die Rate an ypUICC-0, ypUICC-I und ypUICC-II Stadien 14,7\% $(n=26), 23,1 \%(n=41)$ und $27,7 \%(n=49)$. Bei Patienten mit einem ypUICC-II Status lag in 91,8 \% ein günstigeres ypUICC-Ila Tumorstadium vor.

Bereits beim präoperativen Re-Staging konnte ein Ansprechen auf die MMT anhand von 2,8\% $(n=5)$ ycUICC-0, 12,4\% $(n=22)$ ycUICC-I und 27,7\% $(n=49)$ ycUICC-II Stadien vermutet werden. Im Vergleich zur Ausgangsituation mit $4 \%$ cUICC-II und 90,9 \% cUICC-III Stadien war ein deutliches UICC-Downstaging erkennbar. Dabei zeichnete sich der größte Effekt zugunsten $\leq$ cUICC-II Stadien in der Kohorte C (Tabellen 12 und 14) ab. Ob dieser unter OX-basierter MMT (Kohorte B und C) frühzeitig erkennbare Downstaging-Effekt tatsächlich von prognostischer Bedeutung ist, muss an größeren Patientenzahlen, wie z. B. anhand der CAO/ARO/AIO-012-Studie (Fokas et al. 2019), überprüft werden (Abschnitt 4.1.7). Außerdem muss berücksichtigt werden, dass ein MMT-induziertes Downstaging nicht nur die UICC-Stadien abhängige Prognoseabschätzung, sondern auch den Effekt einer adCTx durch die bereits in Abschnitt 3.15 erwähnten Migrationseffekte aus einem reversen Will-Rogers-Phänomen (Fokas et al. 2015) verzerren kann. Es bleibt zudem spekulativ, ob die Ergebnisse des unterschiedlichen Tumoransprechens aus einer frühzeitig erworbenen Therapieresistenz oder aus einer durch die MMT unbeeinflussbaren, primär genetisch festgelegten Malignombiologie resultieren.

Vor diesem Hintergrund ist die Patientengruppe B (Tabelle 24) mit ihren häufiger aufgetretenen FM von besonderem Interesse. Unter Berücksichtigung der Therapiewechsler sollte bei diesen Patienten mit FM nach molekularpathologischen Gemeinsamkeiten (KRAS, BRAF etc.) und nach Unterschieden zu den Therapieansprechern gesucht werden. Eine frühzeitige Identifikation dieser Patienten und 
die Etablierung eines postoperativen Prognose-Scores für den Individualpatienten könnten dazu beitragen, a priori eine an das Rezidivrisiko angepasste MMTStrategie zu wählen. Konkret könnte z. B. einzelnen Patienten mit einem prädizierten ungünstigen Tumoransprechen frühzeitig eine adCTx mit FOLFOXIRI oder FOLFIRI +/- moAk angeboten werden.

Andererseits muss durch Langzeitbeobachtungen geklärt werden, ob jene Fälle mit einem frühzeitig ermittelten Therapieansprechen ( $\leq$ ypUICC-Ila Stadien) tatsächlich eine bessere Prognose besitzen. Dabei ist zu beachten, dass vor allem Patienten mit einer ypCR häufiger eine adCTx ablehnen als Patienten mit einem ypUICC-III Status (Maas et al. 2015). Die Ablehnung der adCTx erfolgt zumeist aufgrund des erwarteten niedrigen Rezidivrisikos (Khrizman et al. 2013). Interessanterweise hatten im eigenen Studienkollektiv sechs Patienten mit den Stadien ypUICC-0 (H49), ypUICC-I (H1, H100) und ypUICC-Ila (H42, H51, H70) keine postoperative adCTx erhalten (Tabelle A1). Bei den Patienten mit einem ypUICC-I Status traten nach 12 Monaten (H100, yG3-Status) und nach 61 Monaten $(\mathrm{H} 1)$ Fernmetastasen auf. Die Patienten verstarben 20 Monate und 79 Monate post operationem am Progress der Tumorerkrankung. Ein weiterer Patient (H51, ypUICC-Ila Stadium) starb nicht tumorbedingt 18 Monate nach TME. Die anderen drei Patienten hatten ein RFS von 91 Monaten (H70, ypUICC-Ila Status), 131 Monaten (H42, ypUICC-Ila Status) und 146 Monaten (H49, ypUICC-0 Status). Aus dieser begrenzten Anzahl an CR-Patienten ohne adCTx ergeben sich die nachfolgenden Fragen.

Kann tatsächlich gefolgert werden, dass je geringer das ypUICC-Stadium ist, desto länger das CSS sein wird?

Sind ypUICC- und pUICC-Stadien prognostisch gleich zu werten?

Profitieren Patienten mit ypUICC-0 und -I Status überhaupt von einer adCTx mit 5-FU oder FOLFOX (Fokas et al. 2015; Maas et al. 2010)?

Kann das Downstaging als unabhängiger Prognosefaktor genutzt werden?

In der ersten globalen Auswertung der CAO/ARO/AIO-94-Studie hatte sich für die präoperative CRT kein signifikanter Vorteil im DFS und OS abgezeichnet (Sauer et al. 2004). Die Langzeitbeobachtung nach > 10 Jahren ergab in Subgruppenana- 
lysen, dass Patienten mit ypUICC-III Stadien (nach präoperativer CRT) im Vergleich zur Kontrollgruppe mit pUICC-III Status ein schlechteres OS aufwiesen. Ebenso zeigten Studienteilnehmer mit einem residuellen ypUICC-II Status ein höheres FM-Risiko und ein geringeres DFS gegenüber jenen Patienten der Kontrollgruppe mit einem pUICC-II Status. Beim globalen Vergleich der Therapiearme ähnelt das UICC-Downstaging einem reversen Will-Rogers-Migrationsphänomen und kann nicht generell als unabhängiger, verlässlicher Endpunkt angesehen werden (Fokas et al. 2015). Allein Patienten mit ypCR- und ypUICC-I Status zeigten ein exzellentes DFS. Diese Beobachtungen entsprechen den eigenen Ergebnissen (Abbildung 13, 18, und 29).

\subsubsection{Einfluss der Therapie-induzierten Tumorregression}

Durch die Intensivierung der präoperativen MMT (z. B. durch Hinzunahme von OX +/- mFOLFOX) kann nicht nur die Rate der ypCR, sondern auch die Rate der nahezu CR ("near complete response", nCR) gesteigert werden (Liang et al. 2019; Rödel et al. 2019; Marco et al. 2018; Garcia-Aquilar et al. 2015). Der prognostische Stellenwert dieser nCR, im Allgemeinen einem ypUICC-I Status entsprechend, ist umstritten (Song et al. 2018; Fokas et al. 2017; Mace et al. 2015; Swellengrebel et al. 2014). Bisher wurde er in nur wenigen Studien unter Anwendung eines standardisierten TRG-Systems (wie z. B. des fünfstufigen TRG-Systems nach Dworak et al. 1997) untersucht (Karagkounis et al. 2019; Suzuki et al. 2017; Kim et al. 2016; Agarwal et al. 2013).

Ähnlich wie in der vorliegenden Dissertation wurde in einer retrospektiven Studie bei 331 LARC-Patienten die Bedeutung einer MMT-induzierten GR (TRG 3/ TRG 4) gegenüber PR (TRG 1/TRG 2) unter einer 7-jährigen Nachsorge geprüft (Song et al. 2018). In der multivariaten Analyse erwies sich die $G R$ als unabhängiger Prädiktor für ein verlängertes DFS und OS. Die Autoren folgerten, dass die GR ein unabhängiger positiver Prognosefaktor sei.

In der eigenen Studienpopulation wurde in univariaten Analysen zunächst festgestellt, dass das DFS (logrank-Test: $p=0,001$ ) und RFS (logrank-Test: $p=0,001$ ) 
der Patienten mit GR (unabhängig von der Tumorgröße und dem Tumorstadium) verlängert war gegenüber Patienten mit einer PR. Der in den univariaten K-MKurven in Tendenz sichtbare Überlebensvorteil bezüglich des CSS für Patienten mit GR (logrank-Test: $p=0,063$ ) ließ sich in der multivariaten Analyse nicht mehr nachvollziehen (Abbildung 29).

\subsection{6 ypL-/V-/Pn-, CRM-, R-Status in Bezug zum CSS}

Nach einer präoperativen CRT werden ein positiver ypL-/V-/Pn-Status, dem positiven EMVI-Status im MRT entsprechend, und eine ypCRM-Positivität als ungünstige Prognosefaktoren angesehen. Sie können mit einer erhöhten Rate an LR (LR: $43 \%$, Gosens et al. 2007) und FM (FM: $29 \%$ bei R0- und $75 \%$ bei R1-/R2Status, Mawdsley et al. 2005) einhergehen. Die klinische Bedeutung dieser vom UICC-Stadium unabhängigen Parameter zeigte sich auch in einer randomisierten Studie (Chand et al. 2014) mit Chirurgen $(n=142)$ und Onkologen $(n=99)$. Diese beiden in die Therapie des Rektumkarzinoms eingebundenen Arztgruppen waren zum Stellenwert einer adCTx für Patienten im Stadium UICC-II und einem weiteren Risikomerkmal, wie z. B. einer im MRT darstellbaren Gefäßinvasion (mrEMVI), befragt worden. Die Mehrheit der Ärzte in beiden Fachgruppen sprach sich für den Einsatz einer adCTx +/- OX aus. Ein Konsens über deren Nutzen konnte allerdings nicht gefunden werden.

In der eigenen Studienpopulation ( $n=177$ Patienten) hatten nur $69,5 \%(n=123)$ aller Studienteilnehmer einen negativen ypL-/V-/Pn-Status. Ein positiver ypL-/V-/ Pn-Status wurde bei jeweils vier Patienten mit einem ypUICC-II Status in den Kohorten A und B diagnostiziert. Zudem wurden bei acht Patienten der Kohorte $A$ und bei fünf Patienten der Kohorte B sowohl ypUICC-III Stadien wie auch positive ypL-/V-/Pn-Status (high-risk Patienten) festgestellt. In der multivariaten Analyse zum CSS zeigte sich entgegen der Einschätzung aus den univariaten Berechnungen, dass eine ypL-/V-/Pn-Positivität nach und unter MMT nicht als unabhängiger Prädiktor für das CSS angesehen werden kann (Abbildung 29).

Ein positiver ypCRM, d. h. eine tumorfreie Distanz von $<2 \mathrm{~mm}$, lag bei $8,5 \%$ $(n=15)$ der Patienten vor (Tabelle 14). In der multivariaten Analyse zeigte sich 
ebenfalls kein signifikanter Einfluss dieser ypCRM-Positivität auf das CSS der Patienten.

Bei allen Überlebenszeitanalysen wurden Patienten mit einem R0-/R1-Status unabhängig vom ypCRM-Status eingeschlossen. Die CHRONICLE-Studie hatte hingegen allein Patienten mit einem CRM von $>1 \mathrm{~mm}$ aufgenommen (Glynne-Jones et al. 2014). In der ADORE-Studie (Hong et al. 2014) waren Patienten mit einem R1-Status von der Randomisation in die adCTx ausgeschlossen worden.

In der PROCTOR-SCRIPT-Studie (Dutch Colorectal Cancer Group, CKTO 200316: ISRCTN36266738) (Breugom et al. 2015a) betrug die R1-Rate $4 \%$; diese lag in der eigenen Studienpopulation bei $2,8 \%$.

\subsubsection{Einfluss der Qualität der TME-Chirurgie}

Die Qualität der TME ist bekanntermaßen ein wichtiger Prognosefaktor (Quirke et al. 2009; Heald 1998; Heald et al 1998). Die Unversehrtheit der chirurgischen Resektatoberfläche und die vollständige Exzision des mesorektalen Weichgewebekompartiments sind Voraussetzung für eine niedrige LR-Rate, ein verbessertes Überleben (Kitz et al. 2018) und die Beurteilung der Effizienz einer adCTx. Insgesamt traten im eigenen untersuchten Patientenkollektiv $(n=177)$ neun $(5,1 \%)$ Lokalrezidive im Beobachtungszeitraum auf. Bei jeweils vier Patienten mit LR hatten die Pathologen initial eine gute $(n=139)$ oder moderate $(n=32)$ TME-Qualität der Rektumresektate festgestellt. Nur in einem Fall war ein LR mit einer schlechten ( $n=6)$ TME-Qualität verbunden.

In den bisher verfügbaren klinischen Studien zur adCTx hatte entweder keine oder eine unzureichende Qualitätsbeurteilung der TME stattgefunden. In der INCRStudie (Sainato et al. 2014) war überhaupt keine TME gefordert worden. In der EORTC-22921-Studie (Bosset et al. 2006) hatten nur $37 \%$ der Studienteilnehmer eine TME erhalten. In der QUASAR-Studie (Quasar 2007) waren keine Angaben zur Art der Operation, zur TME-Qualität und zum CRM gemacht worden. In der PROCTOR-SCRIPT-Studie (Breugom et al. 2015a; Sainato et al. 2014) war in $66,0 \%$ bis $82,7 \%$ der Patienten von einer TME berichtet worden.

In der vorliegenden Untersuchung hatten alle Patienten $(n=177)$ eine TME 
(TARR: $n=126,71,2 \%$; APR: $n=51,28,8 \%$ ) erhalten. Bei jeder TME war sowohl eine chirurgische als auch pathologische Resektatbeurteilung erfolgt. In der großen Mehrzahl wurden die Ergebnisse als optimal/gut (Chirurgie: 79,7 \%, Pathologie: 78,5\%) oder moderat (Chirurgie: 17,5\%; Pathologie: 18,1\%) eingestuft. Nur für wenige Fälle wurde die TME-Qualität als schlecht bewertet (Chirurgie: 2,8 \%; Pathologie: 3,4 \%) (Abschnitt 3.2 Tabelle 13).

\subsubsection{Intervall zwischen Rektumresektion und Start der adCTx}

Bei Kolonkarzinomen im Stadium pUICC-III soll eine adCTx innerhalb von vier bis acht Wochen nach Resektion eingeleitet werden. Eine Verzögerung der adCTx um weitere 4 Wochen kann zu einer signifikanten Reduktion des OS (HR: 1,14; 95\%Cl: 1,10-1,17) und des DFS (HR: 1,14; 95\%-Cl: 1,10-1,18) führen (Biagi et al. 2011). Bei Patienten mit LARC und erfolgter präoperativer CRT wird der Beginn einer adCTx innerhalb von sechs bis acht Wochen nach der Rektumresektion empfohlen (Gresham et al. 2015). Zu einer verzögerten Einleitung der adCTx und damit zu einer potentiellen Verschlechterung der Prognose kann es infolge postoperativer Komplikationen (z. B. aufgrund ausgeprägter Wundheilungsstörungen oder der allgemeinen Co-Morbidität) kommen (Sprenger et al. 2018; Cheung et al. 2009). In dem vorliegenden Projekt wurden die Studienteilnehmer innerhalb von vier bis sechs Wochen der geplanten postoperativen adCTx zugeführt.

\subsubsection{Patienten-Compliance gegenüber adCTx}

Klinische Studien bei Kolonkarzinomen haben eine Compliance der Patienten zwischen $70 \%$ bis $86 \%$ gegenüber einer adCTx gezeigt (Yothers et al. 2011; André et al. 2009; Moertel et al. 1990). Bei Patienten mit Rektumkarzinomen betrug die Compliance zur adCTx lediglich $43 \%$ bis 58 \% (Xu et al. 2017; Chand et al. 2014; Bosset et al. 2006). Selbst eine Reduktion der adCTx konnte die Therapieakzeptanz nicht steigern (Glynne-Jones et al. 2014). In der PROCTOR-SCRIPT-Studie wurde nach präoperativer CRT und TME zwar von einer Patientencompliance von 
74 \% gegenüber der adCTx mit 5-FU berichtet (Breugom et al. 2015a), allerdings führte sie zu keinem Überlebensvorteil in den ypUICC-II und -III Stadien. In der ADORE-Studie betrug die Compliance der Patienten gegenüber der adCTx $95 \%$ im Kontrollarm ( $n=141,5-F U$ + FS) und $97 \%$ in der FOLFOX-Gruppe $(n=141)$ (Hong et al. 2014). Die relative Dosisintensität lag für OX bei 93,2 \% und im Kontrollarm für 5-FU bei 95,0\%; für die kontinuierliche 5-FU-Gabe im FOLFOXRegime bei 87,6 \% und für die 5-FU Bolus-Applikation in der Kontrolle bei 86,6 \%. Während der 38-monatigen Beobachtung (Median; IQR: 26,4-50,6 Monate) traten bei den FOLFOX-Patienten häufiger Nebenwirkungen aller NCI-CTCAE-Grade auf. Es handelte sich in 69,9\% $(n=102)$ der Fälle um Neutropenien (vs. 45,6 \%, $n=68 ; p=0,001)$ und in 26,0\% $(n=38)$ um Thrombozytopenien (vs. 2,0\%, $n=3 ; p=0,001)$. Im FOLFOX-Arm wurden nicht-hämatologische Nebenwirkungen wie Fatigue in 28,3 \% ( $n=41)$ vs. $17,4 \%(n=26, p=0,037)$ der Fälle, Nausea in $53,4 \%(n=78)$ vs. $37,6 \%(n=56, p=0,007)$ und sensorische Neuropathien in $70,5 \%(n=103)$ vs. $5,4 \%(n=8, p=0,001)$ der Patienten dokumentiert. Zwischen beiden Therapiearmen gab es jedoch keine signifikanten Unterschiede in den Nebenwirkungen (CTC-Grade 3 und 4). Das 3-Jahre DFS lag in der ITTPopulation der ADORE-Studie unter FOLFOX bei 71,6\% und unter 5-FU bei 62,9 \% (HR: 0,63; 95\%-Cl: 0,41-0,96; $\mathrm{p}=0,03$ ). Den größten Nutzen hatten Patienten mit Rektumkarzinomen der Stadien ypUICC-IIlb und -IIIc (HR: 0,6; 95\%-Cl: 0,37-0,98; $p=0,03)$. Für ypUICC-II Fälle zeigte sich unter adCTx mit FOLFOX keine Verbesserung (HR: 0,74; 95\%-Cl: 0,33-1,66; $p=0,47$ ).

In der eigenen Studienpopulation betrug die Compliance zur adCTx allein bezogen auf die Patientenanzahl ohne Therapiereduktionen 71,2 \% (Tabelle 20). Bezogen auf die Gesamtzahl der postoperativen CTx-Applikationen lag die Compliance bei $77,3 \%$ (Tabelle 23). Die Compliance unterschied sich deutlich zwischen Kohorte A (5-FU + FS) mit 88,5 \% und Kohorte B (mFOLFOX) mit 57,6 \% (Tabelle 20). Ein anderes Bild zeigten die erfassten Toxizitäten. Die Kohorte B wies zwar absolut eine höhere Anzahl von Toxizitäten auf, aber der Anteil an NCl-CTC $\geq$ Grad 3 (intestinale NWK, Fieber, Leuko-/Thrombozytopenie, Thrombose) betrug lediglich $4,8 \%$ gegenüber $7,4 \%$ in der Kohorte $A$. Dies war als Ergebnis einer früheren und höheren Anzahl an Dosisanpassungen zu werten (Tabelle 23). Interessanter- 
weise zeigte sich für Kohorte $\mathrm{C}$ ein hoher Grad an Therapiecompliance (Tabelle 23) bei einem geringeren Anteil an CTC $\geq$ Grad 3 Nebenwirkungen (Tabellen 18 und 19, Summe: $n=13$ ) sowohl gegenüber der Kohorte A (Tabellen 16 und 21, Summe $n=19$ ) als auch der Kohorte B (Tabellen 17 und 22, Summe: $n=42$ ).

Diese eigenen Daten entsprechen den Ergebnissen aus dem Therapiearm B der CAO/ARO/AIO-12-Studie, in dem eine präoperative MMT mit mFOLFOX-CTx analog zur Kohorte C eingesetzt worden war (Fokas et al. 2019).

\subsubsection{Patientenalter - wer profitiert?}

In der CAO/ARO/AIO-04-Studie war mit Hilfe von STEPP-Analysen (subpopulation treatment effect pattern plot analyses) das Alter der 1.232 Patienten als kontinuierliche Variable für das Überleben und die Auswirkungen der OX-haltigen adCTx modelliert worden (Hofheinz et al. 2018c). Nach einer medianen Beobachtung von 50 Monaten zeigten die < 60-jährigen Patienten unter der OX-haltigen präoperativen CRT und adCTx geringere LR- und FM-Raten sowie ein besseres DFS und OS $(p=0,044)$ im Vergleich zu Patienten mit einer 5-FU Monotherapie. Zudem hatten mit OX behandelte Patienten zwischen dem 40. und 70. Lebensjahr ein besseres DFS gegenüber älteren Studienteilnehmern. Aus dieser Beobachtung folgerten die Autoren, dass zwar < 60-jährige Patienten signifikante Überlebensvorteile durch $\mathrm{OX}$ hätten, nicht jedoch Patienten > 70 Lebensjahre.

In der vergleichbaren Patientenpopulation der Kohorten A bis $C$ konnte der berichtete Überlebensvorteil für die Gruppe der $\leq 60$-Jährigen nach Behandlung mit OX nicht beobachtet werden (Tabelle A7). 


\subsection{Ergebnisse im Kontext zu publizierten klinischen Studien}

Die Ergebnisse der ADORE-Studie wiesen erstmals darauf hin, dass Rektumkarzinom-Patienten mit einem residuellen ypUICC-II oder ypUICC-III Status von einer adCTx mit FOLFOX Vorteile im DFS haben können. Weitere explorative Subgruppenanalysen ergaben, dass insbesondere Patienten mit ypUICC-IIlb- und IIlcStatus oder PR (TRG 1 und TRG 2) profitieren.

In den eigenen K-M-Überlebenskurven zeigte sich für Patienten mit ypUICC-0 bis -II Status ein sehr gutes DFS von 72 Monaten (ypUICC-0: $\geq 80 \%$, ypUICC-I und -II: $\geq 60 \%$ ) unabhängig von der durchgeführten perioperativen MMT. Demgegenüber lagen das DFS im ypUICC-III Stadium bei $50 \%$ und im ypUICC-IV Stadium bei unter $20 \%$. Im CSS erreichten die Patienten mit ypUICC-0 bis -II Status zu mindestens 80 \% ein CSS von 72 Monaten (Abschnitte 3.10.1 und 3.10.3).

\subsubsection{Stellenwert von OX in der adCTx beim Rektumkarzinom}

Die Bedeutung einer adCTx nach primärer Resektion lokal fortgeschrittener Rektumkarzinome ist durch klinische Studien belegt. In der QUASAR-Studie (ISRCTN 82375386) wurden z. B. 3.329 Patienten mit KRK nach der primären Resektion entweder in einen alleinigen Beobachtungsarm oder zu einer Fluoropyrimidin-haltigen adCTx randomisiert. Die adCTx erbrachte einen Überlebensvorteil für Patienten mit Rektumkarzinomen (HR: 0,68; 95\%-Cl: 0,48-0,96), nicht jedoch für Patienten mit Kolonkarzinomen (HR: 0,83; 95\%-Cl: 0,65-1,07) (Quasar 2007). In einer Metaanalyse von 9.785 Patienten mit Rektumkarzinomen zeigte sich (Petersen et al. 2012), dass eine Fluoropyrimidin (FP)-basierte adCTx das DFS (HR: 0,75; 95\%-Cl: 0,68-0,83) und OS (HR: 0,83; 95\%-Cl: 0,76-0,91) steigern kann im Vergleich zur alleinigen Nachbeobachtung.

Demgegenüber wird der Stellenwert einer adCTx nach präoperativer CRT und TME weiterhin sehr kontrovers diskutiert. Entgegen der früheren Zwischenergebnisse haben die 10-Jahresdaten der EORTC-22921-Studie keinen signifikanten Überlebensvorteil (DFS, OS) für Patienten nach einer adCTx bestätigen können 
(Bosset et al. 2014). In der PROCTOR-SCRIPT-Studie, die eine adCTx mit FP gegenüber einer alleinigen Nachbeobachtung bei Rektumkarzinom-Patienten mit (größtenteils) vorausgegangener RT und TME-Resektion untersuchte, konnte kein Vorteil für eine adCTx im 10-Jahre DFS und OS aufgezeigt werden (Breugom et al. 2015a). Eine Metaanalyse von vier randomisierten Studien mit insgesamt 1.196 Patienten, die eine präoperative Therapie vor der Rektumresektion erhalten hatten, ergab ebenfalls keinen Hinweis auf verbesserte Überlebensparameter durch die adCTx mit FP (Breugom et al. 2015b). Im Gegensatz dazu wies eine retrospektive, gepoolte Analyse aus fünf randomisierten Studien mit insgesamt 2.795 Patienten nach präoperativer CRT darauf hin, dass eine adCTx in der 10-jährigen Nachbeobachtung zwar die lokale Tumorkontrolle und das OS verbessern kann, nicht jedoch das CSS (Valentini et al. 2011).

In Anbetracht der anhaltenden Kontroverse um den Stellenwert einer adCTx +/OX müssen die oben genannten Ergebnisse mit aktuellen Phase-II/-III Studien zur perioperativen MMT +/- OX verglichen werden. So zeigte z. B. die CAO/ARO/AIO04-Studie, dass eine präoperative CRT mit OX und die adCTx mit FOLFOX gegenüber einer 5-FU Monotherapie im 3-Jahre DFS überlegen sind $(75,9 \%$ vs. 71,2 \%; HR: 0,79; 95\%-Cl: 0,64-0,98; $p=0,03$ ) (Rödel et al. 2015). Allerdings ist, wie im Abschnitt 4.1 beschrieben, die Aussagekraft zur adCTx limitiert und die Nachbeobachtungszeit noch zu kurz.

Die multizentrische PETACC-6-Studie (ClinicalTrials.gov identifier: NCT00766155) hatte zwar das gleiche Design wie die CAO/ARO/AIO-04-Studie, setzte jedoch Capecitabin anstatt von infusionalem 5-FU ein. Nach den bisher allein in Abstractform verfügbaren Ergebnissen konnte in dieser Studie kein Überlebensvorteil durch die Zugabe von OX in der perioperativen MMT erreicht werden (Schmoll et al. 2018). Auch in dieser Studie war die Randomisation prätherapeutisch erfolgt.

Andererseits setzten die CAO/ARO/AIO-04- und PETACC-6-Studie standardisierte Vorgehensweisen in der präoperativen CRT und Rektumchirurgie um. In der bereits genannten ADORE-Studie (Abschnitt 4.1.9) waren derartige Standards weder in der präoperativen RT/CRT noch in der Rektumchirurgie a priori festgelegt und überprüft worden. Der Fokus der ADORE-Studie lag allein im Vergleich von zwei 
adCTx-Regimen. Aufgrund dieser unterschiedlichen Therapieansätze und der damit verbundenen Einflussfaktoren ist eine abschließende Beurteilung der OXhaltigen adCTx z. Zt. nicht möglich.

\subsubsection{OX in der adCTx bei $\geq$ ypUICC-II Rektumkarzinomen}

In der oben genannten multizentrischen, randomisierten und kontrollierten ADORE-Phase-II-Studie erbrachte die adCTx mit FOLFOX ein besseres 6-Jahre DFS gegenüber 5-FU + FS bei Patienten mit potentiell kurativ resezierten ypUICC-II und ypUICC-III Rektumkarzinomen. Gegenüber früheren Analysen, die eine Steigerung der 3-Jahre OS-Rate unter FOLFOX ergeben hatten, konnte in der Langzeit-Nachbeobachtung kein signifikanter Unterschied in der 6-Jahre OS Rate bestätigt werden. Allerdings zeigte sich in der Subgruppenanalyse, dass Patienten mit ypN2-Status und schlechter Tumorregression von der adCTx mit FOLFOX im 6-Jahre OS profitierten (Hong et al. 2019; 2014). Sowohl die ADORE-Studie wie auch die o. g. CAO/ARO/AIO-04-Studie zeigten, dass durch eine adCTx mit OX das DFS gesteigert werden kann. Allerdings konnte bisher keine Verbesserung des OS nachgewiesen werden. Somit muss in weiteren Studien geprüft werden, welche Patienten mit welchen residuellen Tumorstadien oder mit welchem Grad an CRT-induzierter Tumorregression von einer adCTx profitieren könnten.

In der multivariaten Analyse der eigenen Patientenpopulation zeigte sich kein Vorteil im CSS für Patienten mit > ypUICC-II Tumorstadien (Abbildung 29).

\subsubsection{OX in der adCTx bei < ypUICC-II Rektumkarzinomen}

Bisher wird beim LARC nach präoperativer CRT eine adCTx mit 5-FU + FS als Standard angesehen, unabhängig vom erreichten ypUICC-Status (Rödel et al. 2015; Hofheinz et al. 2012; Sauer et al. 2012; Bosset et al. 2006; Gerard et al. 2003). Diese Sichtweise hat sich aus den bisherigen klinischen Phase-II/-III Studien ergeben.

In der multivariaten Analyse der eigenen Patientenpopulation zeigte sich ein signi- 
fikanter Vorteil im CSS für Patienten mit $\leq$ ypUICC-II Stadien unter MMT (Abbildung 29). Zudem ließ sich für Patienten mit < ypUICC-II Tumorstadien feststellen, dass gerade in Kohorte $\mathrm{C}$ eine hohe Compliance und ein mit Kohorte $\mathrm{A}$ vergleichbares, geringes Therapie-assoziiertes Nebenwirkungsspektrum vorlag (Abschnitt 4.1.9). Auch im CSS zeichnete sich ein Vorteil für das intensivierte Therapieregime der Kohorte C ab. Gegenüber der Kohorte B zeigten Patienten der Kohorte C eine höhere Therapiecompliance und geringere Anzahl an NCI-CTC Grad 3 und Grad 4 Toxizitäten. Insbesondere Patienten mit ypUICC-0 und -I Status profitierten im OS von der intensivierten MMT (präoperative CRT und mFOLFOX-CTx). Somit stellt sich die Frage nach einer adCTx für diese Patienten eigentlich nicht mehr.

In der Literatur ist der Stellenwert einer adCTx bei Patienten mit ypCR nach präopativer CRT weiterhin unklar geblieben. Zwei aktuelle retrospektive Kohortenstudien mit Patienten im ypCR-Status zeigten positive Auswirkungen einer adCTx mit Fluoropyrimidinen auf das OS (Chang 2018; Dossa et al. 2018; Polanco et al. 2018). In der Studie von Polanco et al. (2018) wurden 2.764 Patienten (Alter: MW $60,0 \pm 12,3$ Jahre; $60 \%$ m, $40 \%$ w) aus der US National Cancer Database (2006 bis 2012) mit cUICC-II/-III Rektumkarzinomen und einem ypCR-Status (ypTONOM0) nach präoperativer CRT analysiert. Unter Anwendung der Propensity-Score-Methode wurde das OS der Patienten mit adCTx $(n=741)$ gegenüber Patienten unter alleiniger Nachbeobachtung $(n=741)$ verglichen. Die Patientengruppe mit adCTx zeigte ein besseres OS (HR: 0,50; 95\%-Cl: 0,32-0,79) und die 1-, 3- und 5-Jahre OS-Raten betrugen 99,7\%, 97,1\% und 94,7\% (gegenüber 99,2\%, 93,6\% und 88,4\% der Gruppe ohne adCTx; $p=0,005)$. In Subgruppenanalysen hatten Patienten mit cT3/cT4-Tumoren und cN-positivem Status die größten Vorteile von der adCTx (HR: 0,47; 95\%-Cl: 0,25-0,91) (Polanco et al. 2018). Die Autoren folgerten, dass die adCTx bei Patienten mit ypCR Status das OS verbessern kann.

In einer weiteren, von kanadischen Chirurgen durchgeführten Kohortenstudie, wurde der Nutzen einer adCTx bei Patienten mit einem ypCR-Status (aus der National Cancer Database, 2006 bis 2012) unter Berücksichtigung des cN-Status untersucht (Dossa et al. 2018). In dieser Studie wurde ebenfalls die Propensity- 
Score-Methode eingesetzt und das OS mit Hilfe von K-M-Überlebenskurven und Cox-Hazard-Modellen in den Patientengruppen verglichen. Es wurden 2.455 Rektumkarzinom-Patienten (Alter: MW 59,5 Jahre; 59,8 \% m, 40,2\% w) mit einem ypCR Status nach präoperativer CRT und Rektumresektion in die Gruppe A mit adCTx ( $n=667$ Patienten) und $>8$ Wochen Nachbeobachtung gegenüber Gruppe $B(n=1788)$ ohne adCTx unterteilt. Während einer medianen Nachbeobachtung von 3,1 Jahren (IQR: 1,94-4,40 Jahre) hatten Patienten mit adCTx ein längeres OS (HR: 0,44; 95\%-Cl: 0,28-0,70). Unter Berücksichtigung des cN-Status ergab sich, dass allein Patienten mit einem im Staging positiven Nodalstatus durch die adCTx ein besseres OS erreicht hatten (HR: 0,24; 95\%-Cl: 0,10-0,58). Die Autoren folgerten, dass eine adCTx bei Patienten mit einer ypCR gerechtfertigt sei. Sie könne zu einem verbesserten OS führen. Insbesondere Patienten mit einem im Staging als Nodal-positiv eingeschätztem Rektumkarzinom scheinen zu profitieren. Zugleich räumten die Autoren Limitationen in ihrer retrospektiven, nichtrandomisierten Untersuchung ein, z. B. die fehlenden Angaben zu applizierten CTx-Zyklen, zur CTx-Dosierung und Adhärenz der Patienten, die fehlenden Informationen über FM, LR und Todesursachen sowie zur Ereignisanzahl innerhalb der bisherigen Nachbeobachtungszeit (Dossa et al. 2018).

Die klinische Bewertung der o. g. retrospektiven Studien wird erschwert durch das Fehlen einer postoperativen Randomisation in eine adCTx vs. alleinige Nachbeobachtung und durch die genutzte Propensity-Score-Methode (Chang 2018). Der tatsächliche Überlebensvorteil durch eine adCTx im ypCR-Status scheint deutlich geringer zu sein. Zudem müssen z. B. bei Einsatz einer OX-haltigen adCTx deren Nebenwirkungen (z. B. PNP) berücksichtigt werden (Chang 2018).

Die aktuelle S3-Leitlinie Kolorektales Karzinom (2019) formuliert (S. 179)

„[...] Eine Empfehlung für oder gegen eine adjuvante Chemotherapie nach erfolgter neoadjuvanter Radiochemotherapie kann auf Grundlage der vorhandenen Datenlage beim Rektumkarzinom nicht geben werden. [...]“.

Diese allgemeine Aussage ist für den Kliniker wenig hilfreich und Ausdruck der anhaltenden Kontroverse. Die indirekt gestellte Forderung nach eindeutigen Ergebnissen aus zahlenmäßig großen, randomisierten klinischen Therapiestudien 
setzt aber den unbedingten Willen von den Patienten, den behandelnden Ärzten und klinischen Institutionen zur konsequenten Teilnahme an derartigen Studien voraus. Insbesondere die Frage nach dem Stellenwert der adCTx im ypCR-Status scheint derzeit in Deutschland kaum realisierbar zu sein.

\subsection{Schlussfolgerung und Ausblick}

Die Ergebnisse an 177 Patienten mit Rektumkarzinomen der klinischen Stadien cUICC-II bis -IV rechtfertigen den Einsatz von OX in der perioperativen Multimodaltherapie. Die Patienten zeigten unter der MMT mit OX eine signifikant höhere Rate an UICC-Downstaging und ein signifikant verlängertes Überleben, sofern postoperativ ein $\leq$ ypUICC-II Status erreicht wurde. Die OX-assoziierten Nebenwirkungen und NCI-CTCAE-Toxizitäten waren durch frühzeitige Dosismodifikationen und geringfügige Verlängerungen der therapiefreien Intervalle klinisch gut beherrschbar.

In Bezug auf das Tumoransprechen und einzelne Überlebensparameter ist eine um OX erweiterte CRT +/- adCTx gegenüber einer MMT mit 5-FU-Monotherapie vorzuziehen. Die präoperative MMT mit CRT (RT + 5-FU + OX) und drei Applikationen mFOLFOX (Kohorte $C$ ) stellt ein gut verträgliches, hocheffizientes, mit einer hohen Tumorregression und sehr guten Patienten-Compliance einhergehendes innovatives Behandlungsregime zugunsten von $\leq$ ypUICC-II Tumorstadien dar. Diese eigenen Ergebnisse stimmen mit den aktuell publizierten Ergebnissen der CAO/ARO/AIO-012-Phase-2b-Studie der GRCSG überein (Fokas et al. 2019).

In der CAO/ARO/AIO-12-Studie wurden zwei sogenannte „totale neoadjuvante CRT-Regime“ (TNT, total neoadjuvant treatment) nach dem pick-the winnerPrinzip für zukünftige Phase-III Studien hinsichtlich ihrer Durchführbarkeit, Sicherheit und einer auf $25 \%$ postulierten ypCR-Rate (ypTONOMO Gx R0) geprüft. Patienten mit LARC der unteren Rektumdrittel erhielten im Therapiearm A präoperativ drei Zyklen mFOLFOX gefolgt von einer CRT (RT + 5-FU + OX) und TME. Patienten im Therapiearm B wurden (analog der Kohorte C der eigenen Patientenpopulation) zuerst mit der präoperativen CRT $(\mathrm{RT}+5-\mathrm{FU}+\mathrm{OX})$ und anschließend mit 
drei Zyklen mFOLFOX und TME behandelt. Aktuell waren 306 Patienten auswertbar (Gruppe A: $n=156$, Gruppe B: $n=150$ ). Die CRT-assoziierten CTC-Grad 3/4 Toxizitäten waren für die Patientengruppe $B(27 \%)$ niedriger gegenüber der Gruppe A (37\%). Die Compliance gegenüber der CRT war in Gruppe B (97\%, $87 \%$ und $93 \%$ ) höher als in der Gruppe A (91\%, $78 \%$ und $76 \%$ ). Die TNT konnte in der Patientengruppe $A$ in $92 \%$ der Fälle komplett verabreicht werden gegenüber $85 \%$ für Gruppe B. In der Intention-to-treat-Analyse wurde in der Patientengruppe B eine ypCR-Rate von $25 \%$ erreicht gegenüber $17 \%$ in der Gruppe A. Somit erfüllte allein die TNT der Gruppe B $(p=0,001)$ die vordefinierte Hypothese einer $25 \%$-CR-Rate (Fokas et al. 2019). Aufgrund einer noch unzureichenden Nachbeobachtungszeit konnten keine Aussagen gemacht werden, ob die erhöhte ypCR-Rate der Gruppe B auch tatsächlich mit einem längeren Überleben einhergeht.

In zukünftigen Studien sollte der frühzeitig erhebbare ypUICC-Status zur Applikation einer an das Rezidivrisiko des Patienten angepassten adCTx genutzt werden. Ob dabei Patienten mit einem $\leq$ ypUICC-I Stadium nach TNT (Kohorte $C$ ) noch zusätzlich von dieser adCTx profitieren, ist allerdings stark zu bezweifeln. Vielmehr muss geprüft werden, ob nicht Patienten mit einem GR- und einem ypUICC-II Status von einer adCTx mit FOLFOX oder FOLFIRI Überlebensvorteile haben können. Zudem bedürfen Patienten mit einem PR und/oder > ypUICC-II Status sicherlich einer intensiveren adCTx, z. B. mit FOLFOXIRI-CTx +/- moAk. In jedem Fall sollte in allen Studien zur adCTx auf der Basis des ypUICC-Status eine erneute postoperative Randomisation in Risiko-stratifizierte Therapiearme erfolgen.

Aufgrund der oben genannten Ergebnisse und in Kenntnis der bisher noch nicht publizierten Daten der TransValid-KFO-179/GRCSG-B-Studie sind in Deutschland bereits zwei neue Studien der GRCSG gestartet worden: In der randomisierten Phase-III Studie ACO/ARO/AIO-18.1 // AIO-KRK-0419 (,Preoperative oxaliplatinbased chemoradiotherapy and consolidation chemotherapy versus fluorouracilbased chemoradiotherapy for MRI-defined intermediate and high-risk rectal cancer 
patients: A randomized phase III trial of the German Rectal Cancer Study Group, EudraCT No.: 2018-000876-14") wird die TNT des Therapiearmes B der CAO/ARO/AIO-012-Studie (bzw. der Kohorte C) gegen den bisherigen Standard einer 5-FU-basierten CRT bei durch das pelvine MRT definierten Risikopatienten (intermediäres und hohes Rezidivrisiko) geprüft.

In der randomisierten ACO/ARO/AIO-18.2 // AIO-KRK-0319 Phase-III Studie („Preoperative FOLFOX versus postoperative risk-adapted chemotherapy in patient with locally advanced rectal cancer and low risk for local failure: $A$ randomized phase III trial of the German Rectal Cancer Rectal Study Group, EudraCT No.: 2018-001356-35") wird der Stellenwert der präoperativen FOLFOX-CTx gegenüber einer Risiko-adaptierten postoperativen adCTx bei LARC mit niedrigem Rezidivrisiko untersucht. 


\section{$5 \quad$ Zusammenfassung}

Die präoperative, 5-Fluorouracil(5-FU)-basierte Chemoradiotherapie (CRT) mit nachfolgender totaler mesorektaler Exzision (TME) und additiver Chemotherapie (adCTx) ist die Standardbehandlung lokal fortgeschrittener Rektumkarzinome (LARC). Die Auswirkungen von OX in der MMT sind unter anhaltender Diskussion. Zwischen 07/2006 und 06/2016 erhielten 177 Patienten (55 w, $122 \mathrm{~m}$; medianes Alter: 63 Jahre) mit den LARC-Stadien cUICC-II (4\%), -III (91\%) und -IV (5,1\%) eine präoperative MMT (Kohorte A: $n=64$, Standard-MMT; Kohorte B: $n=63$, MMT + OX; Kohorte C: $n=50$, MMT + OX + mFOLFOX). Die TME- und postoperative Resektatbeurteilung erfolgte nach aktuellen MERCURY- und TNM-/UICCKlassifikationskriterien. Die therapiebegleitende Toxizität wurde nach NCI-CTCAEKriterien (Version 4.03) erfasst. Das beschränkte, erwartete mittlere (restricted mean, rMW) krankheitsfreie (DFS) sowie rezidivfreie (RFS), das krebsspezifische (CSS) und gesamte (OS) Überleben wurden berechnet, durch logrank-Test analysiert und mit der Kaplan-Meier-Methode dargestellt. Für CSS wurde ein CoxProportional-Hazard-Regression-Modell mit ausgewählten Variablen entwickelt (Signifikanzniveau $\alpha=5 \%$ ).

In 98,3\% der Fälle konnte die geplante CRT mit 12,2\% Dosisreduktion verabreicht werden. Postoperativ wurden ein R0-, ein negativer ypCRM- ( $\geq 2 \mathrm{~mm})$ und ein negativer ypL-/V-/Pn-Status in 96,6 \%, 91,5\% und 69,5\% erreicht. Das pathologische Staging ergab yUICC-0 bis -IV Stadien in 14,5\%, 23,1 \%, 27,7 \%, 24,9\% und $9,6 \%$. Die TME-Qualität war in 78,5\% optimal, in $18,1 \%$ moderat und in $3,4 \%$ schlecht. In 54,8\% der Resektate betrug die Tumorlängsausdehnung (ypTE) $\leq 25 \mathrm{~mm}$ und ein gutes Tumoransprechen (GR; TRG 3/TRG 4) war in 53,9\% erreicht worden. Während der präoperativen MMT wurde eine CTC $\geq$ Grad 3 in $4,4 \%$ bei insgesamt 918 Ereignissen dokumentiert. In Kohorte $C$ trat eine CTC $\geq$ Grad 3 in 2,4 \% von 241 Ereignissen (21x Grad 2, 1x Grad 3) während der mFOLFOX-Gaben auf. Nach der TME wurden zehn Patienten ausgeschlossen. Zudem wechselten 11 Patienten die geplante adCTx (9x von Kohorte A zu B, 2x von Kohorte B zu A). 118 Patienten begannen die adCTx. In $100 \%, 98,3 \%$, $96,6 \%$ und $89,8 \%$ konnten alle vier CTx-Zyklen appliziert werden. Eine CTxDosisreduktion erfolgte in 28,8 \% (6x 5-FU, 28x FOLFOX) aller Gaben. Innerhalb von $<3$ Wochen nach 5-FU-CTx trat eine CTC $\geq$ Grad 3 in 7,4\% von 216 Ereig- 
nissen auf (68x Grad 2, 11x Grad 3, 5x Grad 4). Während der FOLFOX-CTx wurde eine CTC $\geq$ Grad 3 in 4,8 \% von 419 Ereignissen festgestellt (118x Grad 2, 18x Grad 3, 1x Grad 4, 1x Grad 5). Insgesamt war die MMT-Compliance der Patienten (Applikationen/Zyklen) mit 87,8\% hoch. Während der 73-monatigen Nachbeobachtung (Median) traten in 31,3\% Fernmetastasen und in 5,4\% Lokalrezidive auf. Das mittlere (rMW) DFS betrug 91,5 Monate (95\%-CI: 82,1-100,9 Monate), das CSS 121,7 Monate (95\%-Cl: 113,8-129,5 Monate) und das OS 105,9 Monate (95\%-Cl: 97,5-114,2 Monate). In univariaten Analysen zeigten sich für die ypUICC-Stadien $(p<0,0009)$, den GR- $(p=0,001)$, ypCRM- $(p=0,002)$ und ypL-/V-/Pn-Status $(p=0,003)$ sowie die ypTE $\leq 25 \mathrm{~mm} \quad(p=0,023)$ signifikante Unterschiede im DFS. In der multivariaten CSS-Analyse (Forest-Plot) ergaben sich keine Unterschiede für die Kohorten B ( $p=0,108$; HR: 2,04; 95\%-Cl: 0,86$4,88)$ und $C(p=0,771$; HR: 1,19; 95\%-Cl: 0,38-3,75) gegenüber der Kohorte $A$ (Referenz). Patienten mit $\leq y p U I C C-I I$ Stadien hatten ein längeres CSS ( $p=0,007$; HR: 0,31; 95\%-Cl: 0,14-0,73).

OX in der perioperativen MMT ist sicher, gut applizierbar und resultiert in einem stärkeren, frühzeitigen Tumoransprechen. Insbesondere die präoperative MMT mit mFOLFOX (Kohorte $\mathrm{C}$ ) ermöglicht eine hohe Tumorregression, gute Compliance und vielversprechende Überlebensergebnisse. Nicht-Ansprecher (> ypUICC-IIIc Stadien) auf die MMT benötigen eine intensivere adCTx, z. B. FOLFOXIRI. 


\section{Anhang}

Im Anhang werden Zusatzinformationen sowie Klassifikationssysteme angegeben. Sie dienen zum näheren Verständnis des Haupttextes.

\subsection{Erläuternde Tabellen}

\begin{tabular}{|c|c|c|c|c|c|c|}
\hline Nr. & $\begin{array}{l}\text { Pat.-Nr., } \\
\text { Kohorte }\end{array}$ & $\begin{array}{l}\text { Alter, } \\
\text { Genus }\end{array}$ & OP & Gründe für nicht-erfolgte adCTx & weiterer Verlauf (Nachsorge): & $\begin{array}{l}\text { ypUICC- } \\
\text { Stadium }\end{array}$ \\
\hline 1 & $\mathrm{H} 1, \mathrm{~A}$ & $58 \mathrm{~J}, \mathrm{~m}$ & APR & postoperative WHS & $\begin{array}{l}\text { RFS: } 61 \text { Monate, ED: pulmonale FM, Status: Tod tumorbedingt, } \\
\text { OS: } 79 \text { Monate }\end{array}$ & 1 \\
\hline 2 & $\mathrm{H} 42, \mathrm{~A}$ & $73 \mathrm{~J}, \mathrm{~m}$ & TARR & postoperative WHS & RFS: 131 Monate, Status: lebt, tumorfrei & lla \\
\hline 3 & $\mathrm{H} 49, \mathrm{~A}$ & $60 \mathrm{~J}, \mathrm{~m}$ & TARR & Patient lehnt adCTx ab & RFS: 146 Monate, Status: lebt, tumorfrei & 0 \\
\hline 4 & $\mathrm{H} 51, \mathrm{~B}$ & $81 \mathrm{~J}, \mathrm{w}$ & TARR & Patient lehnt adCTx ab & \begin{tabular}{|l} 
RFS: 18 Monate, Status: Tod nicht tumorbedingt; Tod nach \\
Herzversagen und cerebralem Insult, OS: 18 Monate
\end{tabular} & lla \\
\hline 5 & $\mathrm{H} 64, \mathrm{~A}$ & $54 \mathrm{~J}, \mathrm{~m}$ & TARR & $\begin{array}{l}2 \text { Tage postoperativ Platzbauch (Nahtbruch), Re-Laparotomie, } \\
\text { postoperative Lungenembolie (LE) }\end{array}$ & $\begin{array}{l}\text { RFS: } 13 \text { Tage, Status: Tod nicht tumorbedingt durch LE, OS: } 13 \\
\text { Tage }\end{array}$ & 1 \\
\hline 6 & $\mathrm{H} 70, \mathrm{~B}$ & $78 \mathrm{~J}, \mathrm{w}$ & TARR & Patient lehnt adCTx ab & RFS: 91 Monate, Status: Tod nicht tumorbedingt, OS: 91 Monate & lla \\
\hline 7 & H96, B & $60 \mathrm{~J}, \mathrm{~m}$ & APR & kein R0-Status, progrediente synchrone FM, R2b-Status bzgl. der FM & RFS: 0 Monate, Status: Tod tumorbedingt, OS: 5 Monate & $\mathrm{IVb}$ \\
\hline 8 & $\mathrm{H} 100, \mathrm{~B}$ & $75 \mathrm{~J}, \mathrm{~m}$ & APR & postoperative WHS, Patient lehnt adCTx ab, ypG3-Status & $\begin{array}{l}\text { RFS: } 12 \text { Monate, Status: Tod tumorbedingt, Progress der } \\
\text { Peritonealkarzinose, OS: } 20 \text { Monate }\end{array}$ & 1 \\
\hline 9 & $\mathrm{H} 113, \mathrm{C}$ & $69 \mathrm{~J}, \mathrm{w}$ & TARR & $\begin{array}{l}\text { synchrone hepatische und peritoneale FM, R2b-Status bzgl. FM, } \\
\text { palliative CTx mit } 3 \text { Zyklen FOLFIRI gestartet }\end{array}$ & $\begin{array}{l}\text { RFS: } 0 \text { Tage, Status: Tod tumorbedingt aufgrund Progress der FM, } \\
\text { OS: } 8 \text { Monate }\end{array}$ & $\mathrm{IVb}$ \\
\hline 10 & $\mathrm{H} 154, \mathrm{~A}$ & $79 \mathrm{~J}, \mathrm{~m}$ & APR & $\begin{array}{l}16 \text { Tage nach APR Kammerflimmern, kardiopulmonale Re- } \\
\text { Stabilisierung, MRSA und multiresistenter Pseudomonas }\end{array}$ & $\begin{array}{l}\text { RFS: } 53 \text { Tage, Status: Tod nicht tumorbedingt aufgrund ARDS und } \\
\text { Sepsis, OS: } 53 \text { Tage }\end{array}$ & lla \\
\hline
\end{tabular}

Tabelle A1: Patienten ohne additive Chemotherapie (adCTx)

*WHS: Wundheilungsstörung, LE: Lungenembolie, ARDS: akutes Atemnot-Syndrom, TARR: tiefe anteriore Rektumresektion, APR: abdominoperineale Rektumresektion; R0: kein Tumorrest, R2b: Resektionsstatus mit makroskopisch und mikroskopisch gesichertem Tumorrest, m: männlich, w: weiblich, UICC: Tumorstadium, ypUICC: histopathologisches Tumorstadium nach erfolgter Chemoradiotherapie/Chemotherapie (CRT/CTx), FM: Fernmetastasen, G: Grading; ED: Erstdiagnose; OS: Gesamtüberleben, RFS: rezidivfreies Überleben, MRSA: Methicillin resistenter Staphylococus aureus 


\begin{tabular}{|c|c|c|c|c|c|c|c|}
\hline Nr. & $\begin{array}{l}\text { Pat.-Nr., } \\
\text { Kohorte }\end{array}$ & $\begin{array}{l}\text { Alter, } \\
\text { Genus }\end{array}$ & "*RT Dosis (\%) & $\begin{array}{l}{ }^{*} \text { CTx Dosis während } \\
\text { CRT ( \%) }\end{array}$ & Grund für die Dosisreduktion & \begin{tabular}{|l} 
Postoperative adCTx, verabreichte Dosis und \\
Therapie, Dosisreduktionen und aufgetretene \\
Nebenwirkungen (NWK) \\
\end{tabular} & $\begin{array}{l}\text { ypUICC- } \\
\text { Stadium }\end{array}$ \\
\hline 1 & $\mathrm{H} 15, \mathrm{~B}$ & $63 \mathrm{~J}, \mathrm{~m}$ & $100 \%$ & 2. Zyklus: 5 -FU $75 \%$ & Portinfekt: CTC-Grad 3 & $\begin{array}{l}\text { adCTx: } 2 \text { Zyklen: mFOLFOX } 100 \% \text { \% } 2 \text { Zyklen: mFOLFOX } \\
50 \% \text {; persistierende Leukopenie: CTC-Grad 1, } \\
\text { HB -Abfall: CTC-Grad } 2\end{array}$ & 1 \\
\hline 2 & $\mathrm{H} 19, \mathrm{~B}$ & $72 \mathrm{~J}, \mathrm{~m}$ & $100 \%$ & 2. Zyklus: OX $75 \%$ & neurologische Symptome: CTC-Grad 2 & $\begin{array}{l}\text { adCTx: } 2 \text { Zyklen: mFOLFOX } 50 \% \text {, OX-PNP; IL-6 Syndrom: CTC- } \\
\text { Grad 2, Patient verw eigerte } 3 \text {. und 4. Zyklus }\end{array}$ & $\|$ \\
\hline 3 & $\mathrm{H} 25, \mathrm{~B}$ & $57 \mathrm{~J}, \mathrm{w}$ & $100 \%$ & 2. Zyklus: mFOLFOX $50 \%$ & Portinfektion: CTC-Grad 3 & adCTx: 4 Zyklen: mFOLFOX $100 \%$ & 1 \\
\hline 4 & $H 28, B$ & $74 \mathrm{~J}, \mathrm{w}$ & $91,7 \%$ & 2 Zyklen: mFOLFOX $100 \%$ & Entscheidung des Radioonkologen & adCTx: 4 Zyklen: mFOLFOX $100 \%$ & ॥I \\
\hline 5 & $\mathrm{H} 445, \mathrm{~B}$ & $50 \mathrm{~J}, \mathrm{w}$ & $100 \%$ & 2. Zyklus: mFOLFOX $50 \%$ & $\begin{array}{l}\text { Leukopenie: CTC-Grad 2, } \\
\text { Diarrhoe: CTC-Grad 2, WHS; } \\
\text { Hb-Abfall: CTC-Grad 2, } \\
\text { AZ: CTC-Grad 2 } \\
\end{array}$ & $\begin{array}{l}\text { adCTx: } 2 \text { Zyklen: mFOLFOX } 80 \text { \%, 3. Zyklus: 5-FU } 100 \% \text { + OX } \\
50 \% \text {, 4. Zyklus: 5-FU } 50 \% \text { und kein OX; } \\
\text { Leukopenie: CTC-Grad 2, Diarrhoe: CTC-Grad 3, } \\
\text { AZ-Abfall: CTC-Grad 3, PNP: CTC-Grad 2 }\end{array}$ & $\|$ \\
\hline 6 & H97, A & $77 \mathrm{~J}, \mathrm{~m}$ & $100 \%$ & $\begin{array}{l}\text { 1. Zyklus: } 5-\mathrm{FU} 40 \% \text {, } \\
\text { kein 2. Zyklus }\end{array}$ & $\begin{array}{l}\text { Koronarspasmen unter 5-FU, } \\
\text { initialer DPD-Test w ar negativ }\end{array}$ & $\begin{array}{l}\text { adCTx: } 2 \text { Zyklen: mit 5-FU } 50 \% \text {, im } 3 . \text { Zyklus: Abbruch der } \\
\text { 5-FU Gabe nach } 25 \% \text { aufgrund erneuter } \\
\text { Koronarspasmen: CTC-Grad } 4 \\
\end{array}$ & IIII \\
\hline 7 & $\mathrm{H} 100, \mathrm{~B}$ & $75 \mathrm{~J}, \mathrm{~m}$ & $100 \%$ & 2. Zyklus: $5-\mathrm{FU}+\mathrm{OX} 50 \%$ & WHS sakral & keine adCTx, protrahierte Wundheilungsstörung & 1 \\
\hline 8 & $\mathrm{H} 170, \mathrm{~B}$ & $56 \mathrm{~J}, \mathrm{w}$ & $57,1 \%$ & $\begin{array}{l}50 \% \text {, 2. Zyklus: mFOLFOX } \\
\text { nicht appliziert }\end{array}$ & $\begin{array}{l}\text { Schmerzen, Ebrechen, Diarrhoe: CTC-Grad } \\
\text { 3, Transaminasen: CTC-Grad 2, } \\
\text { Abbruch auf Entscheid des Radioonkologen }\end{array}$ & $\begin{array}{l}\text { adCTX: 1. und 2. Zyklus: mFOLFOX } 50 \% \text {, 3. Zyklus (1. Gabe): } \\
\text { mFOLFOX 50 \%, 3. Zyklus (2. Gabe): 5- FU } 100 \% \text { + OX } 50 \% \text {, } \\
\text { 4. Zyklus: 5- FU } 100 \% \text { + OX } 50 \% \text {, Strahlenenteritis: CTC- } \\
\text { Grad 3, Diarrhoe: CTC-Grad 2, OX-PNP: CTC-Grad 2, } \\
\text { Leukopenie: CTC-Grad } 1\end{array}$ & $\|$ \\
\hline 9 & $\mathrm{H} 177, \mathrm{~B}$ & $52 \mathrm{~J}, \mathrm{w}$ & $36 \%$ & $\begin{array}{l}50 \% \text {, 2. Zyklus: mFOLFOX } \\
\text { nicht appliziert }\end{array}$ & $\begin{array}{l}\text { Übelkeit, Erbrechen, Diarrhoe: CTC-Grad 3, } \\
\text { Körpergew ichtsreduktion: CTC-Grad 2, } \\
\text { Abbruch auf Entscheid des Radioonkologen }\end{array}$ & adCTx: 4 Zyklen: mFOLFOX $100 \%$ problemlos appliziert & 0 \\
\hline
\end{tabular}

Tabelle A2: Patienten der Kohorten A und B mit Dosisreduktionen in der präoperativen CRT

${ }^{*} \mathrm{RT}(\%)$ : prozentualer Anteil der applizierten RT von der geplanten Gesamtdosis; ${ }^{*} \mathrm{CTx}(\%)$ : prozentualer Anteil der verabreichten CTx während der Radiatio. AZ: Allgemeinzustand, DPD-Test: Dihydropyrimidindehydrogenase, Exon 14-Skipping-Mutation Test, WHS: Wundheilungsstörung, Hb: Hämoglobin; PNP: Polyneuropathie, IL-6: Interleukin-6 Syndrom, CTC-Grad: Common Toxicity Criteria, ab Grad 3: schwerwiegende unerwünschte Ereignisse 


\begin{tabular}{|c|c|c|c|c|c|c|c|c|}
\hline Nr. & $\begin{array}{l}\text { Pat.-Nr, } \\
\text { Kohorte }\end{array}$ & $\begin{array}{l}\text { Alter, } \\
\text { Genus }\end{array}$ & $\begin{array}{c}\text { *RT Dosis } \\
(\%)\end{array}$ & $\begin{array}{c}\text { **CTx Dosis } \\
\text { während } \\
\text { CRT (\%) }\end{array}$ & ${ }^{\star \star \star} \mathrm{CTX}$, nach CRT & Grund für die Dosisreduktion & \begin{tabular}{|l|} 
Postoperative adCTx, \\
verabreichte Dosis und \\
Therapie, Dosisreduktionen \\
und aufgetretene NWK \\
\end{tabular} & $\begin{array}{l}\text { ypUICC- } \\
\text { Stadium }\end{array}$ \\
\hline 1 & $\mathrm{H} 106, \mathrm{C}$ & $58 \mathrm{~J}, \mathrm{~m}$ & $100 \%$ & $100 \%$ & 3 Zyklen: mFOLFOX $75 \%$ & Nierenfunktionseinschränkung: CTC-Grad 1 & $\begin{array}{l}\text { keine adCTx, Krampfanfall, } \\
\text { Hyponatriämie, } \\
\text { Hb-Abfall: CTC-Grad 2; Suizid }\end{array}$ & 1 \\
\hline 2 & $\mathrm{H} 109, \mathrm{C}$ & $69 \mathrm{~J}, \mathrm{~m}$ & $100 \%$ & $\begin{array}{l}\text { 2. Zyklus: } \\
\text { OX } 75 \%\end{array}$ & 3 Zyklen: OX $75 \%+5-F U 100 \%$ & $\begin{array}{l}\text { OX-Reduktion, } \\
\text { Entscheidung durch den Radioonkologen, } \\
\text { Hautexanthem: CTC-Grad 2, } \\
\text { L-6-Syndrom }\end{array}$ & \begin{tabular}{|l|} 
adCTx: \\
3 Zyklen: mFOLFOX $100 \%$, keine \\
relevanten NWK
\end{tabular} & $\|$ \\
\hline 3 & $\mathrm{H} 115, \mathrm{C}$ & $37 \mathrm{~J}, \mathrm{w}$ & $100 \%$ & $100 \%$ & 2. Zyklus: OX $75 \%$ & OX: Leukopenie: CTC-Grad 2 & keine adCTx & 0 \\
\hline 4 & $\mathrm{H} 118, \mathrm{C}$ & $69 \mathrm{~J}, \mathrm{~m}$ & $100 \%$ & $100 \%$ & 3. Zyklus: 5-FU + OX $75 \%$ & OX-PNP: CTC-Grad 2 & keine adCTx & 0 \\
\hline 5 & $\mathrm{H} 134, \mathrm{C}$ & $51 \mathrm{~J}, \mathrm{~m}$ & $100 \%$ & $100 \%$ & 1. Zyklus: OX $75 \%$ & Kachexie: CTC-Grad 2. & keine adCTx & $\|$ \\
\hline 6 & $\mathrm{H} 136, \mathrm{C}$ & $65 \mathrm{~J}, \mathrm{w}$ & $100 \%$ & $100 \%$ & 1. Zyklus: 5 -FU $50 \%+$ OX $100 \%$ & Port-Dysfunktion & keine adCTx & 0 \\
\hline 7 & $\mathrm{H} 142, \mathrm{C}$ & $54 \mathrm{~J}, \mathrm{~m}$ & $100 \%$ & $100 \%$ & 1. bis 3. Zyklus: mFOLFOX $75 \%$ & $\begin{array}{l}\text { ST Hebung im EKG, } \\
\text { Leukopenie: CTC-Grad: } 2\end{array}$ & keine $\operatorname{adCTx}$ & 0 \\
\hline 8 & $\mathrm{H} 144, \mathrm{C}$ & $65 \mathrm{~J}, \mathrm{~m}$ & $100 \%$ & $100 \%$ & 2. und 3. Zyklus: OX $75 \%$ & $\begin{array}{l}\text { Al-Spätinsuffizienz, Intervallverlängerung bei } \\
\text { Leukopenie: CTC-Grad: } 2\end{array}$ & keine adCTx & । \\
\hline 9 & $\mathrm{H} 149, \mathrm{C}$ & $67 \mathrm{~J}, \mathrm{~m}$ & $100 \%$ & $100 \%$ & 1. bis 3. Zyklus: mFOLFOX $75 \%$ & WHS, Al, OX-PNP: CTC-Grad: 2 & keine adCTx & । \\
\hline
\end{tabular}

Tabelle A3: Patienten Kohorte $\mathrm{C}$ mit Dosisreduktion in der präoperativen MMT

${ }^{*} \mathrm{RT}(\%)$ : prozentualer Anteil der applizierten RT von der geplanten Gesamtdosis; ${ }^{*} \mathrm{CTx}(\%)$ : prozentualer Anteil der verabreichten CTx während der RT; ${ }^{* *}$ CTX nach CRT (\%): prozentualer Anteil der präoperativ verabreichten mFOLFOX-CTx in Patienten Kohorte C; die angegebenen Einzelbefunde werden z. T. im Ergebnis- und Diskussionsteil erörtert. 


\begin{tabular}{|c|c|c|c|c|c|c|c|}
\hline Nr. & $\begin{array}{l}\text { Pat.-Nr, } \\
\text { Kohorte }\end{array}$ & $\begin{array}{l}\text { Alter, } \\
\text { Genus }\end{array}$ & $\left|\begin{array}{c}\text { RT Dosis } \\
(\%)\end{array}\right|$ & $\begin{array}{l}{ }^{*} \text { *CTx Dosis } \\
\text { während CRT (\%) }\end{array}$ & $\begin{array}{l}\text { Grund für die Dosisreduktion } \\
\text { während präoperativer Therapie }\end{array}$ & $\begin{array}{l}\text { Postoperative adCTx mit Dosisreduktionen und } \\
\text { aufgetretenen Nebenwirkungen }\end{array}$ & $\begin{array}{l}\text { ypUICC } \\
\text { Stadium }\end{array}$ \\
\hline 1 & $\mathrm{H} 3, \mathrm{~B}$ & $76 \mathrm{~J}, \mathrm{w}$ & $100 \%$ & $100 \%$ & - & \begin{tabular}{|l|} 
adCTx: 3. und 4. Zyklus: OX 0 \%, Diarrhoe CTC-Grad 3, \\
Infektion: CTC-Grad 2, Leuko- und Thrombopenie: CTC-Grad 1, \\
OX-Allergie: CTC-Grad 2, Transaminasen: CTC-Grad 2 \\
\end{tabular} & III \\
\hline 2 & $\mathrm{H} 6, \mathrm{~B}$ & $48 \mathrm{~J}, \mathrm{~m}$ & $100 \%$ & $100 \%$ & - & $\begin{array}{l}\text { adCTx: 4. Zyklus: mFOLFOX Abbruch aufgrund } \\
\text { allergische Reaktion: CTC-Grad } 1\end{array}$ & $\|$ \\
\hline 3 & $\mathrm{H} 14, \mathrm{~A}$ & $74 \mathrm{~J}, \mathrm{w}$ & $100 \%$ & $100 \%$ & & \begin{tabular}{|l} 
adCTx: 4 Zyklen: 5-FU 80 \%, Dyspnoe: CTC-Grad 2, \\
Leukopenie: CTC-Grad 2, Mukositis: CTC-Grad 2 \\
\end{tabular} & $\|$ \\
\hline 4 & $\mathrm{H} 15, \mathrm{~B}$ & $63 \mathrm{~J}, \mathrm{~m}$ & $100 \%$ & $\begin{array}{l}\text { 2. Zyklus: } \\
\text { 5-FU } 75 \%\end{array}$ & Portinfektion: CTC-Grad 3 & $\begin{array}{l}\text { adCTx: 3. und 4. Zyklus: mFOLFOX } 50 \text { \%; } \\
\text { persistierende Leukopenie: CTC-Grad 1, } \\
\text { Diarrhoe: CTC-Grad 2, HB-Abfall: CTC-Grad 2, } \\
\text { Infektion: CTC-Grad 3, Kreatinin-Anstieg: CTC-Grad 2 } \\
\end{array}$ & 1 \\
\hline 5 & $\mathrm{H} 17, \mathrm{~B}$ & $65 \mathrm{~J}, \mathrm{~m}$ & $100 \%$ & $100 \%$ & - & adCTx: 4. Zyklus nicht gegeben, Portinfektion: CTC-Grad 3 & III \\
\hline 6 & $\mathrm{H} 19, \mathrm{~B}$ & $72 \mathrm{~J}, \mathrm{~m}$ & $100 \%$ & $\begin{array}{l}\text { 2. Zyklus: } \\
\text { OX } 75 \%\end{array}$ & $\begin{array}{l}\text { Portinfektion: CTC-Grad 2, } \\
\text { Diarrhoe: CTC-Grad } 3\end{array}$ & $\begin{array}{l}\text { adCTx: 1. und 2. Zyklus: mFOLFOX } 75 \text { \%, Kribbelparästhesien } \\
\text { (PNP); IL-6 Syndrom: Grad 3, Diarrhoe: Grad 2, } \\
\text { HB-Abfall: CTC-Grad 2; Patient verw eigerte 3. und 4. Zyklus }\end{array}$ & $\|$ \\
\hline 7 & H26, B & $54 \mathrm{~J}, \mathrm{~m}$ & $100 \%$ & $100 \%$ & & $\begin{array}{l}\text { adCTx: 2. Zyklus: mFOLFOX } 50 \% \text {, 3. und 4. Zyklus: mFOLFOX } \\
75 \text { \%, Diarrhoe: CTC-Grad 3, KG-Verlust: CTC-Grad 2, } \\
\text { Hb-Abfall: CTC-Grad } 2\end{array}$ & 1 \\
\hline 8 & $\mathrm{H} 33, \mathrm{~A}$ & $61 \mathrm{~J}, \mathrm{~m}$ & $100 \%$ & $100 \%$ & & $\begin{array}{l}\text { adCTx: 4. Zyklus: 5-FU } 80 \% \text {, } \\
\text { ansteigende Kreatininw erte: CTC-Grad } 1\end{array}$ & III \\
\hline 9 & $\mathrm{H} 40, \mathrm{~B}$ & $79 \mathrm{~J}, \mathrm{~m}$ & $100 \%$ & $100 \%$ & - & $\begin{array}{l}\text { adCTx: 3. und 4. Zyklus: mFOLFOX } 75 \text { \%, } \\
\text { Diarrhoe: CTC-Grad } 3\end{array}$ & 1 \\
\hline 10 & $\mathrm{H} 43, \mathrm{~B}$ & $62 \mathrm{~J}, \mathrm{~m}$ & $100 \%$ & $100 \%$ & & $\begin{array}{l}\text { adCTx: 1. und 2. Zyklus: mFOLFOX } 80 \% \text {, 3. Zyklus: mFOLFOX } \\
50 \% \text {, 4. Zyklus: 5-FU } 100 \%+\text { OX } 2 \% \text { bei Abbruch aufgrund } \\
\text { allergischer Reaktion: CTC-Grad 2, PNP: CTC-Grad } 2\end{array}$ & IV \\
\hline 11 & $\mathrm{H} 45, \mathrm{~B}$ & $50 \mathrm{~J}, \mathrm{w}$ & $100 \%$ & $\begin{array}{l}\text { 2. Zyklus: } \\
\text { mFOLFOX } 50 \%\end{array}$ & $\begin{array}{l}\text { Leukopenie: CTC-Grad 2, } \\
\text { Diarrhoe: CTC-Grad 2, WHS, } \\
\text { Hb-Abfall: CTC-Grad 2, } \\
\text { AZ-Abfall: CTC-Grad 2 }\end{array}$ & $\begin{array}{l}\text { adCTX: 1. und 2. Zyklus: mFOLFOX } 75 \text { \%, 3. und 4. Zyklus: } \\
50 \text { \%, Leukopenie: CTC-Grad 2, PNP: CTC-Grad 2, HB-Abfall: } \\
\text { CTC-Grad 2, Diarrhoe: CTC-Grad 3, } \\
\text { AZ-Abfall: CTC-Grad 3 }\end{array}$ & $\|$ \\
\hline
\end{tabular}

Tabelle A4 Teil 1/4 


\begin{tabular}{|c|c|c|c|c|c|c|c|}
\hline Nr. & $\begin{array}{l}\text { Pat.-Nr, } \\
\text { Kohorte }\end{array}$ & $\begin{array}{l}\text { Alter, } \\
\text { Genus }\end{array}$ & $\left|\begin{array}{c}\text { *RT Dosis } \\
(\%)\end{array}\right|$ & $\begin{array}{c}{ }^{*} \text { CTX Dosis } \\
\text { während CRT (\%) }\end{array}$ & $\begin{array}{l}\text { Grund für die Dosisreduktion } \\
\text { während präoperativer Therapie }\end{array}$ & $\begin{array}{l}\text { Postoperative adCTx mit Dosisreduktionen und } \\
\text { aufgetretenen Nebenwirkungen }\end{array}$ & $\begin{array}{l}\text { ypUICC- } \\
\text { Stadium }\end{array}$ \\
\hline 12 & $\mathrm{H} 47, \mathrm{~B}$ & $54 \mathrm{~J}, \mathrm{~m}$ & $100 \%$ & $\begin{array}{l}\text { 1. Zyklus: } \\
\text { 5-FU } 75 \% \\
+ \text { OX } 100 \%\end{array}$ & Defekt der Baxterpumpe & $\begin{array}{l}\text { adCTx: 2. Zyklus: OX } 50 \%+5 \text {-FU } 100 \% \text {, 4. Zyklus: mFOLFOX } \\
50 \% \text { (keine 8. Gabe, bei Lebermetastasen-Op.), } \\
\text { Übelkeit: CTC-Grad 3, Erbrechen: CTC-Grad 2, } \\
\text { Proktitis: CTC-Grad 2, OX-Allergie: CTC-Grad } 2\end{array}$ & $\|$ \\
\hline 13 & $\mathrm{H} 52, \mathrm{~B}$ & $67 \mathrm{~J}, \mathrm{w}$ & $100 \%$ & $100 \%$ & - & $\begin{array}{l}\text { adCTx: 1. Zyklus: OX } 50 \% \text { + 5-FU } 100 \% \text {, 2. und 3. Zyklus: } \\
\text { mFOLFOX 50 \%, 4. Zyklus: OX } 0 \% \text { + 5-FU 50 \%, Leukopenie: } \\
\text { CTC-Grad 2, OX-Allergie: CTC-Grad 2, PNP: CTC-Grad 2, } \\
\text { IL-6-Syndrom: CTC-Grad 3, kardiale Arrhythmie: CTC-Grad } 2\end{array}$ & 0 \\
\hline 14 & $\mathrm{H} 55, \mathrm{~B}$ & $65 \mathrm{~J}, \mathrm{w}$ & $100 \%$ & $100 \%$ & - & $\begin{array}{l}\text { adCTx: 1. bis 4. Zyklus: mFOLFOX 5-FU: } 100 \% \text { + OX } 50 \% \text {, } \\
\text { Strahlenkolitis: CTC-Grad } 3\end{array}$ & 0 \\
\hline 15 & $\mathrm{H} 58, \mathrm{~B}$ & $35 \mathrm{~J}, \mathrm{w}$ & $100 \%$ & $100 \%$ & - & $\begin{array}{l}\text { adCTx: 1. bis 4. Zyklus: mFOLFOX 5-FU } 100 \%+\text { OX } 75 \% \text {, } \\
\text { Diarrhoe und Erbrechen: CTC-Grad } 2\end{array}$ & I \\
\hline 16 & H62, B & $73 \mathrm{~J}, \mathrm{w}$ & $100 \%$ & $100 \%$ & - & adCTx: 2. bis 4. Zyklus: 5-FU+OX $75 \%$, PNP: CTC-Grad 2 & 1 \\
\hline 17 & $\mathrm{H} 63, \mathrm{~B}$ & $79 \mathrm{~J}, \mathrm{~m}$ & $100 \%$ & $100 \%$ & - & $\begin{array}{l}\text { adCTx: äquivalente Gabe von Capecitabin zu 5-FU, w egen } \\
\text { Portinfekt und Portexplantation, OX } 0 \text { \% bei V.a. allergische } \\
\text { Reaktion w ährend der präoperativen CRT: CTC-Grad: } 3, \\
\text { A-Insuffizienz }\end{array}$ & 1 \\
\hline 18 & H66, A & $73 \mathrm{~J}, \mathrm{w}$ & $100 \%$ & $100 \%$ & & $\begin{array}{l}\text { adCTx: 1. Zyklus: 5-FU } 100 \% \text {, CTx auf Wunsch der Pat. } \\
\text { beendet (w eg. Begleiterkrankungen), Leukopenie: CTC-Grad 2, } \\
\text { Hb-Abf all bei Hämorrhagie: CTC-Grad 2, Diarrhoe: CTC-Grad 2, } \\
\text { Thrombose: CTC-Grad 2, Exitus: Lokalrezidiv }\end{array}$ & III \\
\hline 19 & $H 72, B$ & $67 \mathrm{~J}, \mathrm{~m}$ & $100 \%$ & $100 \%$ & - & $\begin{array}{l}\text { adCTx: nach 1. Zyklus: mFOLFOX Änderung der Therapie (Op.) } \\
\text { bei Tumorprogress (Diagnose von LM), PNP: CTC-Grad } 2\end{array}$ & IV \\
\hline 20 & $H 74, B$ & $60 \mathrm{~J}, \mathrm{~m}$ & $100 \%$ & $100 \%$ & - & $\begin{array}{l}\text { adCTx: keine 2. Gabe im 4. Zyklus mFOLFOX auf Wunsch des } \\
\text { Patienten }\end{array}$ & $\|$ \\
\hline
\end{tabular}

Tabelle A4 Teil 2/4 


\begin{tabular}{|c|c|c|c|c|c|c|c|}
\hline $\mathrm{Nr}$. & $\begin{array}{l}\text { Pat.-Nr, } \\
\text { Kohorte }\end{array}$ & $\begin{array}{l}\text { Alter, } \\
\text { Genus }\end{array}$ & $\begin{array}{c}\text { "RT Dosis } \\
(\%)\end{array}$ & $\begin{array}{l}{ }^{* *} \text { CTx Dosis } \\
\text { während CRT (\%) }\end{array}$ & $\begin{array}{l}\text { Grund für die Dosisreduktion } \\
\text { während präoperativer Therapie }\end{array}$ & $\begin{array}{l}\text { Postoperative adCTx m it Dosisreduktionen und } \\
\text { aufgetretenen Nebenwirkungen }\end{array}$ & $\begin{array}{l}\text { ypUICC- } \\
\text { Stadium }\end{array}$ \\
\hline 21 & $H 79, A$ & $64 \mathrm{~J}, \mathrm{~m}$ & $100 \%$ & $100 \%$ & - & $\begin{array}{l}\text { mFOLFOX 1. Zyklus: } 100 \% \text {, 2. und 3. Zyklus: 5-FU } 100 \% \text {, OX } \\
75 \% \text {;. Zyklus: eine Applikation mit 5-FU } 100 \% \text {, kein OX, } \\
\text { Abbruch auf Wunsch des Patienten }\end{array}$ & III \\
\hline 22 & $\mathrm{H} 84, \mathrm{~B}$ & $62 \mathrm{~J}, \mathrm{~m}$ & $100 \%$ & $100 \%$ & - & $\begin{array}{l}\text { adCTx: 4. Zyklus: 5-FU } 100 \% \text { + OX } 0 \text { \% auf Wunsch des } \\
\text { Patienten }\end{array}$ & 1 \\
\hline 23 & H86, A & $72 \mathrm{~J}, \mathrm{w}$ & $100 \%$ & $100 \%$ & - & $\begin{array}{l}\text { adCTx: 1. und 2. Zyklus: 5-FU, } \\
\text { Abbruch w egen Granulozytopenie: CTC-Grad 4, } \\
\text { AZ-Verschlechterung: CTC-Grad } 3\end{array}$ & 1 \\
\hline 24 & $H 88, B$ & $65 \mathrm{~J}, \mathrm{~m}$ & $100 \%$ & $100 \%$ & - & $\begin{array}{l}\text { adCTx: 1. und 2. Zyklus: mFOLFOX } 75 \% \text {, 3. und 4. Zyklus: } \\
\text { mFOLFOX } 50 \% \text {, 2.Gabe im 4. Zyklus nicht appliziert, w eg. lleus } \\
\text { und Exitus }\end{array}$ & IV \\
\hline 25 & H91, B & $73 \mathrm{~J}, \mathrm{w}$ & $100 \%$ & $100 \%$ & - & adCTx: 1. bis 4. Zyklus: mFolf ox $75 \%$, Hypertonus: CTC-Grad 2 & $\|$ \\
\hline 26 & $\mathrm{H} 94, \mathrm{~B}$ & $66 \mathrm{~J}, \mathrm{~m}$ & $100 \%$ & $100 \%$ & - & $\begin{array}{l}\text { adCTx: mFOLFOX 1. bis 4. Zyklus: OX } 50 \text { \%, Diarrhoen: CTC- } \\
\text { Grad 3, AZ-Verschlechterung: CTC-Grad 3, } \\
\text { Gew ichtsreduktion: CTC-Grad 2, Diarrhoe: CTC-Grad } 3\end{array}$ & 1 \\
\hline 27 & $\mathrm{H} 97, \mathrm{~A}$ & $77 \mathrm{~J}, \mathrm{~m}$ & $100 \%$ & $\begin{array}{l}\text { 5-FU } \\
\text { 1. Zyklus: } 40 \% \text {, } \\
\text { 2. Zyklus: } 0 \%\end{array}$ & $\begin{array}{l}\text { Koronarspasmen unter 5-FU, } \\
\text { initialer DPD-Test: negativ }\end{array}$ & $\begin{array}{l}\text { adCTx: } 2 \text { Zyklen: 5-FU } 50 \% \text {, im 3. Zyklus: Abbruch der 5-FU } \\
\text { Gabe nach } 25 \% \text { aufgrund erneuter Koronarspasmen }\end{array}$ & III \\
\hline 28 & $\mathrm{H} 101, \mathrm{~B}$ & $53 \mathrm{~J}, \mathrm{~m}$ & $100 \%$ & $100 \%$ & - & $\begin{array}{l}\text { adCTx: } 4 \text { Zyklen: mFOLFOX } 75 \text { \%, Thrombozytopenie: CTC-Grad } \\
\text { 2, Strahlenproktitis: CTC-Grad 1, Soorösophagitis: CTC-Grad 3, } \\
\text { Hb-Abfall: CTC-Grad 3, Lebermetastasen -Progress und Exitus, } \\
\text { Nachsorge w urde vom Patienten nicht w ahrgenommen }\end{array}$ & III \\
\hline 29 & H161, B & $61 \mathrm{~J}, \mathrm{~m}$ & $100 \%$ & $\begin{array}{l}\text { mFOLFOX } \\
\text { 1. Zyklus: } 100 \% \\
\text { 2. Zyklus: OX } 0 \% \\
+5 \text {-FU } 50 \%\end{array}$ & $\begin{array}{l}\text { Erbrechen, Diarrhoe: CTC-Grad 3, } \\
\text { terminale Niereninsuffizienz, } \\
\text { Kreatinin-Anstieg: CTC-Grad 4, } \\
\text { DPD-Test: negativ }\end{array}$ & $\begin{array}{l}\text { adCTx: 1. Zyklus: OX } 50 \% \text { + } 5 \text {-FU } 100 \% \text {, 2. Zyklus (1 Gabe): } \\
\text { mFOLFOX } 50 \% \text { kardiale NW: CTC-Grad 3, 2 Wochen nach } \\
\text { Abbruch der adCTx plötzlicher Herztod: CTC-Grad 5, Obduktion: } \\
\text { Defekt im Hiss-Bündel, Sick-Sinus-Syndrom }\end{array}$ & III \\
\hline
\end{tabular}

Tabelle A4 Teil 3/4 


\begin{tabular}{|c|c|c|c|c|c|c|c|}
\hline Nr. & $\begin{array}{l}\text { Pat.-Nr, } \\
\text { Kohorte }\end{array}$ & $\begin{array}{l}\text { Alter, } \\
\text { Genus }\end{array}$ & $\mid \begin{array}{c}\text { *RT Dosis } \\
(\%)\end{array}$ & $\begin{array}{c}{ }^{* *} \text { CTx Dosis } \\
\text { während CRT (\%) }\end{array}$ & $\begin{array}{l}\text { Grund für die Dosisreduktion } \\
\text { während präoperativer Therapie }\end{array}$ & $\begin{array}{l}\text { Postoperative adCTx mit Dosisreduktionen und } \\
\text { aufgetretenen Nebenwirkungen }\end{array}$ & $\begin{array}{l}\text { ypUICC- } \\
\text { Stadium }\end{array}$ \\
\hline 30 & H166, B & $66 \mathrm{~J}, \mathrm{~m}$ & $100 \%$ & $100 \%$ & - & $\begin{array}{l}\text { adCTx: 3. und 4. Zyklus: OX } 0 \% \text {, Wundheilungsstörung, } \\
\text { Pelviner Abszess: CTC-Grad } 4\end{array}$ & $\|$ \\
\hline 31 & H169, B & $44 \mathrm{~J}, \mathrm{w}$ & $100 \%$ & $100 \%$ & - & $\begin{array}{l}\text { adCTx: 3. und 4. Zyklus: mFOLFOX } 75 \% \text {, } \\
\text { Übelkeit: CTC-Grad 1, Erbrechen: CTC-Grad 3, } \\
\text { PNP: CTC-Grad 2, Leukopenie: CTC-Grad } 1 \\
\end{array}$ & $\|$ \\
\hline 32 & $\mathrm{H} 170, \mathrm{~B}$ & $56 \mathrm{~J}, \mathrm{w}$ & $57,1 \%$ & $\begin{array}{l}\text { mFOLFOX } \\
\text { 1. Zyklus: } 50 \% \text {, } \\
\text { 2. Zykklus: nicht } \\
\text { appliziert }\end{array}$ & $\begin{array}{l}\text { Übelkeit, Erbrechen: CTC-Grad 3, } \\
\text { Schmerzen: CTC-Grad 3, } \\
\text { Transaminasenanstieg: CTC-Grad 2, } \\
\text { Abbruch der präoperativen CRT, } \\
\text { Entscheid des Radioonkologen }\end{array}$ & $\begin{array}{l}\text { adCTx: 1. und 2. Zyklen: mFOLFOX } 50 \text { \%, 3. Zyklen (1 Gabe): } \\
\text { mFOLFOX 50 \%, 3. Zyklus (2.Gabe): 5- FU } 100 \% \text { + OX } 50 \% \text {, } \\
\text { 4. Zyklus: 5- FU } 100 \% \text { + OX } 50 \% \text {, } \\
\text { Strahlenenteritis: CTC-Grad 3, Diarrhoe: CTC-Grad 2, } \\
\text { PNP: CTC-Grad 2, Leukopenie: CTC-Grad 1 }\end{array}$ & $\|$ \\
\hline 33 & $\mathrm{H} 172, \mathrm{~A}$ & $68 \mathrm{~J}, \mathrm{w}$ & $100 \%$ & $100 \%$ & - & $\begin{array}{l}\text { adCTx: 2. bis 4. Zyklus: 5-FU } 50 \% \text {, } \\
\text { Übelkeit und Erbrechen: CTC-Grad 2, Leukopenie: CTC-Grad 4, } \\
\text { Hb-Abf all: CTC-Grad 2, Mukositis: CTC-Grad } 2\end{array}$ & IV \\
\hline 34 & H173, B & $63 \mathrm{~J}, \mathrm{w}$ & $100 \%$ & $100 \%$ & - & $\begin{array}{l}\text { adCTx: 1. Zyklus: mFOLFOX } 100 \% \text {, 2. bis 4. Zyklus: OX } 50 \%+ \\
\text { 5-FU } 100 \% \text {, OX-Allergie: CTC-Grad 3, Übelkeit: CTC-Grad 2, } \\
\text { Fieber: CTC-Grad } 1\end{array}$ & $\|$ \\
\hline 35 & $\mathrm{H} 174, \mathrm{~B}$ & $69 \mathrm{~J}, \mathrm{~m}$ & $100 \%$ & $100 \%$ & - & $\begin{array}{l}\text { adCTx: 1. und 2. Zyklus: mFOLFOX } 100 \% \text {, Abbruch der CTx } \\
\text { auf Wunsch des Patienten, Diarrhoe: CTC-Grad 3, fieberhafter } \\
\text { bronchopulmonaler Infekt vom Patient verschleppt: CTC-Grad 3, } \\
\text { AZ-Verschlechterung: CTC-Grad } 3\end{array}$ & III \\
\hline
\end{tabular}

Tabelle A4: Patienten der Kohorten A und B mit Dosisreduktion der adCTx

Die verabreichte Dosis an postoperativer additiver Chemotherapie (adCTx) sowie der CTx während der präoperativen CRT (Einzelfälle) sind in Prozent (\%) der geplanten Dosis angegeben; (\%) *: Anteil der applizierten Radiatio von der geplanten Gesamtdosis; \%**: prozentualer Anteil der verabreichten CTx während der Radiatio 


\begin{tabular}{|c|c|c|c|c|c|c|c|c|c|}
\hline \multirow{2}{*}{$\begin{array}{l}\mathrm{n}=177 \text { Patienten, } \\
\text { Nebenwirkungen } \\
\text { in CTC-Grade } \geq 1\end{array}$} & \multicolumn{2}{|c|}{ Gesamt } & \multicolumn{7}{|c|}{$\begin{array}{c}\text { Kohorten A, B und C } \\
\text { Toxizitätsgrade (NCI-CTCAE-Version 4.03) }\end{array}$} \\
\hline & Ereignisse* & $\%$ & 1 & 2 & 3 & 4 & 5 & $\geq 2$ & $\geq 3$ \\
\hline \multicolumn{10}{|c|}{ Unter präop. CRT incl. neoadj.CTx } \\
\hline Geschmacksinn/-irritation & 8 & 0,9 & 8 & - & - & - & - & - & - \\
\hline Schmerz & 21 & 2,3 & 15 & 5 & 1 & - & - & 6 & 1 \\
\hline Fatigue & 22 & 2,4 & 20 & 2 & - & - & - & 2 & - \\
\hline Hämoglobin & 149 & 16,2 & 129 & 18 & 2 & - & - & 20 & 2 \\
\hline Leukozyten & 80 & 8,7 & 59 & 18 & 3 & - & - & 21 & 3 \\
\hline Thrombozyten & 41 & 4,5 & 40 & 1 & - & - & - & 1 & - \\
\hline konstitutionelle Symptome ${ }^{* *}$ & 25 & 2,7 & 22 & 3 & - & - & - & 3 & - \\
\hline Kreatinin & 4 & 0,4 & 4 & - & - & - & - & - & - \\
\hline Haut & 125 & 13,6 & 70 & 50 & 5 & - & - & 55 & 5 \\
\hline Stomatitis/Mukositis & 7 & 0,8 & 6 & - & 1 & - & - & 1 & 1 \\
\hline Zystitis & 68 & 7,4 & 48 & 17 & 2 & 1 & - & 20 & 3 \\
\hline Proktitis & 136 & 14,8 & 72 & 60 & 4 & - & - & 64 & 4 \\
\hline intestinale NWK & 103 & 11,2 & 64 & 29 & 10 & - & - & 39 & 10 \\
\hline gastrale NWK & 46 & 5,0 & 28 & 13 & 5 & - & - & 18 & 5 \\
\hline renale NWK & 3 & 0,3 & 3 & - & - & - & - & - & - \\
\hline neurologische NWK & 47 & 5,1 & 41 & 6 & - & - & - & 6 & - \\
\hline kardiale NWK & 1 & 0,1 & 1 & - & - & - & - & - & - \\
\hline Fieber/Infektion & 12 & 1,3 & 6 & 3 & 3 & - & - & 6 & 3 \\
\hline Alopezie & 2 & 0,2 & 2 & - & - & - & - & - & - \\
\hline Allergische Reaktion & 3 & 0,3 & 1 & 1 & 1 & - & - & 2 & 1 \\
\hline $\mathrm{AZ}$ & 2 & 0,2 & 2 & - & - & - & - & - & - \\
\hline Dyspnoe & - & - & - & - & - & - & - & - & - \\
\hline IL-6-Syndrom & 1 & 0,1 & - & 1 & - & - & - & 1 & - \\
\hline Neutrophile & - & - & - & - & - & - & - & - & - \\
\hline Thrombose (TVT) & - & - & - & - & - & - & - & - & - \\
\hline Hepar/Transaminasen, Bilirubin & - & - & - & - & - & - & - & - & - \\
\hline Hand-Fuß-Syndrom & 1 & 0,1 & - & 1 & - & - & - & 1 & - \\
\hline Sonstige & 11 & 1,2 & 5 & 4 & 2 & - & - & 6 & 2 \\
\hline Summe & 918 & 100,0 & 646 & 232 & 39 & 1 & - & 272 & 40 \\
\hline
\end{tabular}

Tabelle A5: Akute Nebenwirkungen unter präoperativer MMT (Kohorten A, B, C)

*Ereignisse: jedes Ereignis einer akuten hämatologischen und/oder Organ-Toxizität wurde mit dem höchsten CTC-Grad für die Gesamtpopulation ( $n=177$ Patienten) dokumentiert, die während der Behandlung und drei Wochen nach der präoperativen CRT aufgetreten waren; **Ernährungszustand und Kognition wurden unter „konstitutionelle Symptome" zusammengefasst. Farblich hervorgehoben werden in der Tabelle die prozentual häufigsten klinischen Symptome; zudem werden höhergradige Toxizitätsgrade zusammengefasst aufgeführt ( $\geq$ Grad 3 bzw. bei Leukozyten $\geq$ Grad 2), da sie häufig die Begründung für Dosisreduktionen darstellten. 


\begin{tabular}{|c|c|c|c|c|c|c|c|c|c|}
\hline \multirow{2}{*}{$\begin{array}{l}\mathrm{n}=118 \text { Patienten (as-treated) } \\
\text { Nebenwirkungen } \\
\text { in CTC-Grade } \geq 1\end{array}$} & \multicolumn{2}{|c|}{ Gesamt } & \multicolumn{7}{|c|}{$\begin{array}{l}\text { Kohorten A und B } \\
\text { Toxizitätsgrade (NCl-CTCAE Version 4.03) }\end{array}$} \\
\hline & Ereignisse* & $\%$ & 1 & 2 & 3 & 4 & 5 & $\geq 2$ & $\geq 3$ \\
\hline \multicolumn{10}{|l|}{ Unter postop. CTx } \\
\hline Geschmacksinn/-irritation & 3 & 0,5 & 1 & 2 & - & - & - & 2 & - \\
\hline Schmerz & 17 & 2,7 & 8 & 8 & 1 & - & - & 9 & 1 \\
\hline Fatigue & 7 & 1,1 & 6 & 1 & - & - & - & 1 & - \\
\hline Hämoglobin & 98 & 15,4 & 75 & 22 & 1 & - & - & 23 & 1 \\
\hline Leukozyten & 51 & 8,0 & 32 & 17 & - & 2 & - & 19 & 2 \\
\hline Thrombozyten & 14 & 2,2 & 13 & - & - & 1 & - & 1 & 1 \\
\hline konstitutionelle Symptome ${ }^{* *}$ & 30 & 4,7 & 22 & 7 & 1 & - & - & 8 & 1 \\
\hline Kreatinin & 35 & 5,5 & 28 & 6 & - & 1 & - & 7 & 1 \\
\hline Haut & 10 & 1,6 & 7 & 3 & - & - & - & 3 & - \\
\hline Stomatitis/Mukositis & 15 & 2,4 & 7 & 8 & - & - & - & 8 & - \\
\hline Zystitis & 2 & 0,3 & 1 & 1 & - & - & - & 1 & - \\
\hline Proktitis & 20 & 3,1 & 7 & 13 & - & - & - & 13 & - \\
\hline intestinale NWK & 46 & 7,2 & 18 & 21 & 7 & - & - & 28 & 7 \\
\hline gastrale NWK & 100 & 15,7 & 74 & 22 & 4 & - & - & 26 & 4 \\
\hline renale NWK & 15 & 2,4 & 7 & 8 & - & - & - & 8 & - \\
\hline neurologische NWK & 50 & 7,9 & 39 & 9 & 2 & - & - & 11 & 2 \\
\hline kardiale $\mathrm{NWK}^{\star * *}$ & 7 & 1,1 & 2 & 3 & - & 1 & 1 & 5 & 2 \\
\hline Fieber/Infektion & 28 & 4,4 & 14 & 7 & 7 & - & - & 14 & 7 \\
\hline Alopezie & 4 & 0,6 & 3 & 1 & - & - & - & 1 & - \\
\hline Allergische Reaktion & 8 & 1,3 & 3 & 5 & - & - & - & 5 & - \\
\hline $\mathrm{AZ}$ & 2 & 0,3 & - & 2 & - & - & - & 2 & - \\
\hline Dyspnoe & 9 & 1,4 & 6 & 2 & 1 & - & - & 3 & 1 \\
\hline IL-6-Syndrom & 3 & 0,5 & 1 & 2 & - & - & - & 2 & - \\
\hline Neutrophile & 1 & 0,2 & - & 1 & - & - & - & 1 & - \\
\hline Thrombose (TVT) & 7 & 1,1 & 1 & 3 & 3 & & & 6 & 3 \\
\hline Hepar/Transaminasen, Bilirubin & 38 & 6,0 & 33 & 5 & - & - & - & 5 & - \\
\hline Hand-Fuß-Syndrom & - & - & - & - & - & - & - & - & - \\
\hline Sonstige & 15 & 2,4 & 5 & 7 & 2 & 1 & - & 10 & 3 \\
\hline Summe & 635 & 100,0 & 413 & 186 & 29 & 6 & 1 & 222 & 36 \\
\hline
\end{tabular}

Tabelle A6: Akute Nebenwirkungen unter adCTx (Kohorten A und B)

Die Therapie erfolgte nach Komplettierung der präoperativen Multimodaltherapie für Patienten der Kohorten A und B $(n=118)$; ${ }^{*}$ Ereignisse: jedes Ereignis einer akuten hämatologischen und/oder Organ-Toxizität wurde mit dem höchsten CTC-Grad dokumentiert, die während der Behandlung und drei Wochen nach präoperativer CRT aufgetreten waren; **Ernährungszustand und Kognition wurden unter „konstitutionelle Symptome“ zusammengefasst. ${ }^{* * *}$ Der Patient mit dem Toxizitätsgrad 5 verstarb 14 Tage (Sick-Sinus-Syndrom) nach dem zweiten Zyklus der postoperativen adCTx mit mFOLFOX in $75 \%$ Dosierung. Die initiale DPD-Testung war bei diesem Patienten unauffällig gewesen. Farblich hervorgehoben werden die Toxizitätsgrade mit dem häufigsten klinischen Symptomkomplex (z. B. $\geq$ Grad 3 bzw. bei Leukozyten $\geq$ Grad 2 mit der möglichen Konsequenz einer Dosisreduktion). 


\begin{tabular}{|c|c|c|c|c|c|c|c|c|}
\hline Gesamt* & & \multicolumn{2}{|c|}{ Kohorte A } & \multicolumn{2}{|c|}{ Kohorte B } & \multicolumn{2}{|c|}{ Kohorte C } & \multirow[t]{2}{*}{ Altersgruppe } \\
\hline $\mathrm{n}$ & & $\mathrm{n}$ & $\%$ & $\mathrm{n}$ & $\%$ & $\mathrm{n}$ & $\%$ & \\
\hline 153 & Anzahl & 19 & 12,4 & 25 & 16,3 & 21 & 13,7 & \multirow{12}{*}{ bis 60 Jahre } \\
\hline 151 & Ro & 19 & 100,0 & 25 & 100,0 & 19 & 90,5 & \\
\hline 2 & R1 & - & - & - & - & 2 & 9,5 & \\
\hline 0 & $\mathrm{R} 2$ & - & - & - & - & - & 0 & \\
\hline 8 & TRG $0+1$ & 2 & 10,5 & 1 & 4,0 & 1 & 4,8 & \\
\hline 118 & TRG $2+3$ & 14 & 73,7 & 20 & 80,0 & 13 & 62,0 & \\
\hline 27 & TRG 4 & 3 & 15,8 & 4 & 16,0 & 7 & 33,3 & \\
\hline 25 & pCR (ypTONO) & 3 & 15,8 & 4 & 16,0 & 5 & 23,8 & \\
\hline 104 & DFS 3 Jahre & 15 & 78,9 & 16 & 64,0 & 13 & 61,9 & \\
\hline 124 & OS 3 Jahre & 17 & 89,5 & 21 & 84,0 & 14 & 66,7 & \\
\hline 149 & LR & 18 & 94,7 & 23 & 92,0 & 21 & 100,0 & \\
\hline 115 & FM & 15 & 78,9 & 16 & 64,0 & 16 & 76,2 & \\
\hline
\end{tabular}

\begin{tabular}{|c|c|c|c|c|c|c|c|}
\hline & \multicolumn{2}{|c|}{ Kohorte A } & \multicolumn{2}{|c|}{ Kohorte B } & \multicolumn{2}{|c|}{ Kohorte C } & \multirow[t]{2}{*}{ Altersgruppe } \\
\hline & $\mathrm{n}$ & $\%$ & $n$ & $\%$ & $n$ & $\%$ & \\
\hline Anzahl & 13 & 8,5 & 20 & 13,1 & 18 & 11,8 & \multirow{12}{*}{$\begin{array}{l}>60 \text { bis } \\
70 \text { Jahre }\end{array}$} \\
\hline Ro & 13 & 100,0 & 20 & 1000,0 & 18 & 1000,0 & \\
\hline R1 & - & - & - & - & - & 0,0 & \\
\hline R2 & - & - & - & - & - & - & \\
\hline TRG $0+1$ & 1 & 7,7 & 0 & 0,0 & 2 & 11,1 & \\
\hline TRG $2+3$ & 9 & 69,2 & 17 & 85,0 & 13 & 72,2 & \\
\hline TRG 4 & 3 & 23,1 & 3 & 15,0 & 3 & 16,7 & \\
\hline pCR (ypTONO) & 3 & 23,1 & 3 & 15,0 & 3 & 16,7 & \\
\hline DFS 3 Jahre & 10 & 76,9 & 13 & 65,0 & 11 & 61,1 & \\
\hline OS 3 Jahre & 10 & 76,9 & 16 & 80,0 & 12 & 66,7 & \\
\hline LR & 13 & 100,0 & 20 & 100,0 & 18 & 100,0 & \\
\hline FM & 10 & 76,9 & 16 & 80,0 & 15 & 83,3 & \\
\hline
\end{tabular}

\begin{tabular}{|c|c|c|c|c|c|c|c|}
\hline & \multicolumn{2}{|c|}{ Kohorte A } & \multicolumn{2}{|c|}{ Kohorte B } & \multicolumn{2}{|c|}{ Kohorte C } & \multirow[t]{2}{*}{ Altersgruppe } \\
\hline & $\mathrm{n}$ & $\%$ & $\mathrm{n}$ & $\%$ & $n$ & $\%$ & \\
\hline Anzahl & 18 & 11,8 & 12 & 7,8 & 7 & 4,6 & \multirow{12}{*}{ > 70 Jahre } \\
\hline Ro & 18 & 100,0 & 12 & 100,0 & 7 & 100,0 & \\
\hline R1 & - & - & - & - & - & - & \\
\hline $\mathrm{R} 2$ & - & - & - & - & - & - & \\
\hline TRG $0+1$ & 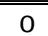 & 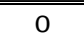 & 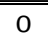 & 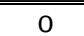 & 1 & 14,3 & \\
\hline TRG $2+3$ & 16 & 88,9 & 11 & 91,7 & 5 & 71,4 & \\
\hline TRG 4 & 2 & 11,1 & 1 & 8,3 & 1 & 14,3 & \\
\hline pCR (ypTONO) & 2 & $\overline{111,1}$ & 1 & 8,3 & 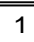 & 1414,3 & \\
\hline DFS 3 Jahre & 13 & 72,2 & 8 & "66,7 & 5 & 71,4 & \\
\hline OS 3 Jahre & 18 & 100,0 & 10 & 83,3 & 6 & 85,7 & \\
\hline LR & 17 & 94,4 & 12 & 100,0 & 7 & 100,0 & \\
\hline FM & 13 & 72,2 & 8 & 66,7 & 6 & 85,7 & \\
\hline
\end{tabular}

Tabelle A7: Residual-, TRG-Status, Rezidive, 3-Jahre DFS und OS nach Alter

Angegeben sind die Ergebnisse aus den Kohorten A, B und C nach Altersgruppen; einbezogen sind nur Patienten, bei denen die präoperative und postoperative MMT nach dem Intention-to-treat-Prinzip identisch waren. Es wurden Therapiewechsler der Kohorten A und B sowie die beiden Pat. mit postoperativer CTx aus Kohorte $\mathrm{C}$ nicht berücksichtigt; so wurde der Einfluss von Veränderungen der Therapieregime ausgeschlossen. R: Residualstatus; TRG: diese Einteilung der Tumorregression nach Dworak et al. (1997) erfolgte zur besseren Vergleichbarkeit mit den Daten der CAO/ARO/AIO-04-Studie analog der Publikation (Hofheinz et al. 2018c); pCR: (histo-)pathologisch komplette Remission; LR: ohne Lokalrezidiv, FM: ohne Fernmetastase. ${ }^{*}$ Gesamt: Anzahl der Fälle (n) aller Kohorten und Altersgruppen 


\subsection{Tumorgenese der KRK}

Sporadische kolorektale Karzinome entstehen nach der „Adenom-KarzinomSequenz" Hypothese (Fearon und Vogelstein 1990) aus Dickdarmpolypen (Adenomen) der Darmschleimhaut. Eine mehrstufige Abfolge von Mutationen führt zur Entartung von normaler Mukosa zu Adenomen und schließlich zu einem unkontrollierten, invasiven und verdrängendem Zellwachstum (Karzinom). Die genetischen und chromosomalen Veränderungen werden durch Mutationen im Onkogen KRAS (kirsten rat sarcoma viral oncogene) und in den Tumorsupressorgenen APC (Adenomatous-polyposis-coll), DCC (deleted in colorectal carcinoma) und Tumorprotein p53 (TP53) ausgelöst. Diese Mutationen erklären jedoch nur z. T. die KRKTumorgenese; es wird mittlerweile von einer multifaktoriellen Tumorgenese ausgegangen (Li und Lai 2009).

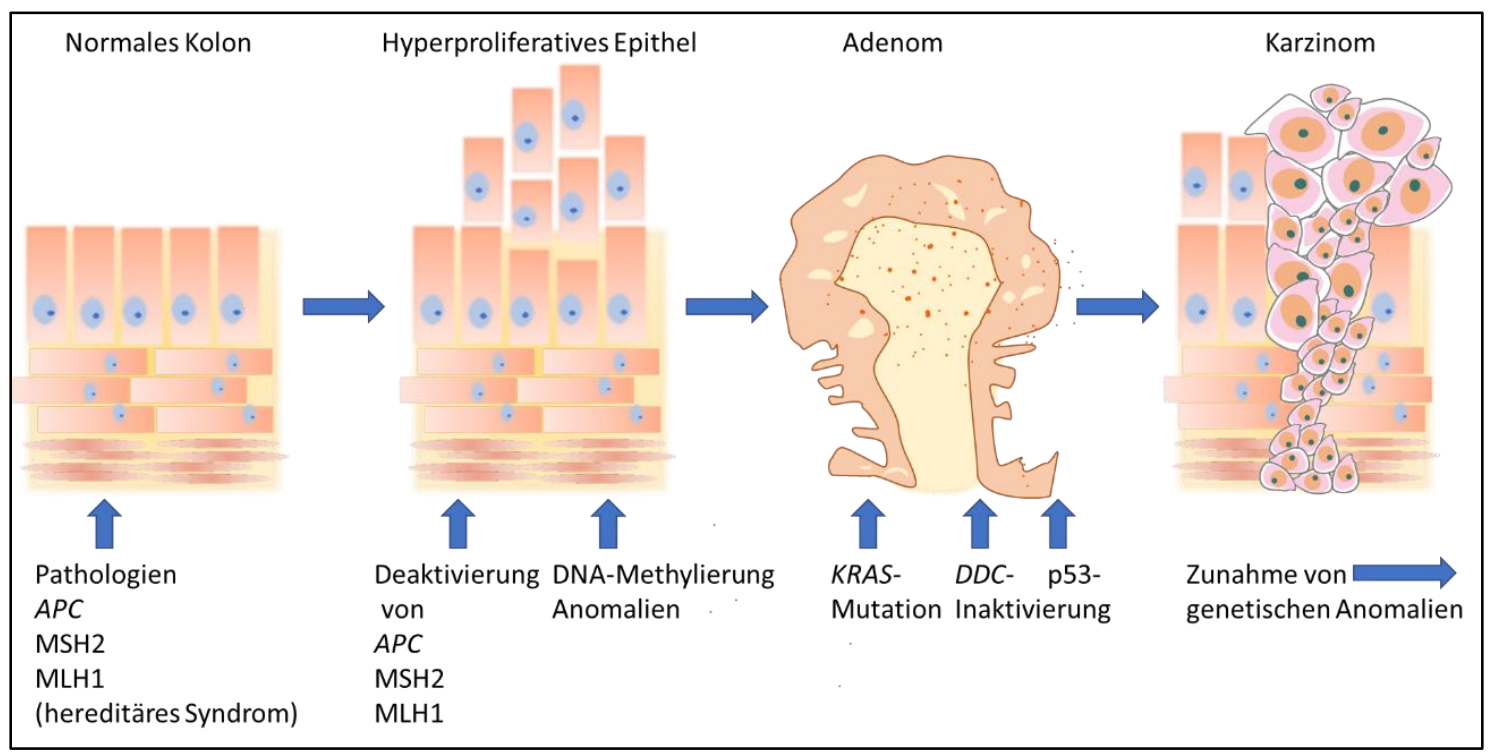

Abbildung A1: Adenom-Karzinom-Sequenz

Eigene Darstellung nach Vogelstein et al. 1988, modifiziert aus Toribara und Sleisenger 1995 


\subsection{Erbliche Pathogenese und weitere Risikofaktoren der KRK}

Eine erbliche (hereditäre) Pathogenese liegt bei etwa $5 \%$ der KRK vor, wie z. B. beim HNPCC-Syndrom (hereditary nonpolyposis colon cancer), das auch LynchSyndrom genannt wird. Molekulargenetische Untersuchungen an diesen polypösen, hereditären KRK und an Patienten mit familiärer adenomatöser Polyposis (FAP, 0,5-1 \%) zeigten (Munding und Tannapfel 2009), dass neben der o. g. Adenom-Karzinom-Sequenz auch andere Metabolisierungswege relevant sind.

Bei $12 \%$ aller sporadischen KRK liegt eine Mikrosatelliten-Instabilität (MSI) vor. Bei HNPCC-Patienten ist die MSI in 80 bis $90 \%$ der Fälle im Tumor nachweisbar. Mikrosatelliten sind DNA-Sequenzen, die zahlreich und repetitiv (10 bis 60x) über das gesamte Genom verteilt sind. Bei der DNA-Amplifikation können in repetitiven DNA-Sequenzen Replikationsfehler auftreten, die üblicherweise von MismatchRepair(MMR)-Genen repariert werden. Liegen allerdings Funktionsstörungen der MMR-Gene vor, so ergeben sich Replikationsfehler mit einer erhöhten Mutationsrate von Genen, die eine Malignomentstehung fördern. Gegenüber Kolonkarzinomen scheinen derartige Metabolisierungswege bei der Tumorgenese von Rektumkarzinomen von geringerer Bedeutung zu sein. Es ist bekannt, dass Karzinome des distalen Sigmas, des rektosigmoidalen Übergangs und des Rektums eine 10fach niedrigere MSI-Inzidenz aufweisen im Vergleich zu höher gelegenen Kolonkarzinomen (Miyakura et al. 2001; Thibodeau et al. 1993).

Karzinome, die auf dem Boden eines Adenoms entstehen, weisen häufig eine chromosomale Instabilität auf. Durch Insertion und Deletion kommt es zum Verlust der Heterozygotie (loss of heterozygosity, kurz: LOH). Die Elimination der Wildtypkopie eines Gens kann dabei sowohl eine Inaktivierung als auch eine Aktivierung des Genprodukts bewirken. Bei der Tumorgenese von KRK sind sowohl Tumorsuppressorgene auf Chromosom 5q, 17q und $18 q$ als auch die Onkogene KRAS und $M Y C$ beteiligt. Ist $M Y C$ mutiert führt es durch die Verstärkung bestehender Genexpressionsprogramme zu Tumorbildung. Der Name MYC bezieht sich auf die Krankheit „Myelocytomatose“ und bezeichnet sowohl einen Transkriptionsfaktor, als auch das dazugehörige Proto-Onkogen, das sich auf Chromosom 8, Genlokus 8q24.21, befindet. Etwa $60 \%-75 \%$ aller sporadischen KRK weisen eine chromo- 
somale Instabilität (CIN) auf (Gervaz et al. 2001). Beim Rektumkarzinom wurde schon vor 20 Jahren eine hohe Inzidenz an CIN beschrieben (Aaltonen 1998).

In Tabelle A8 sind weitere Risikofaktoren für das Auftreten von KRK angegeben.

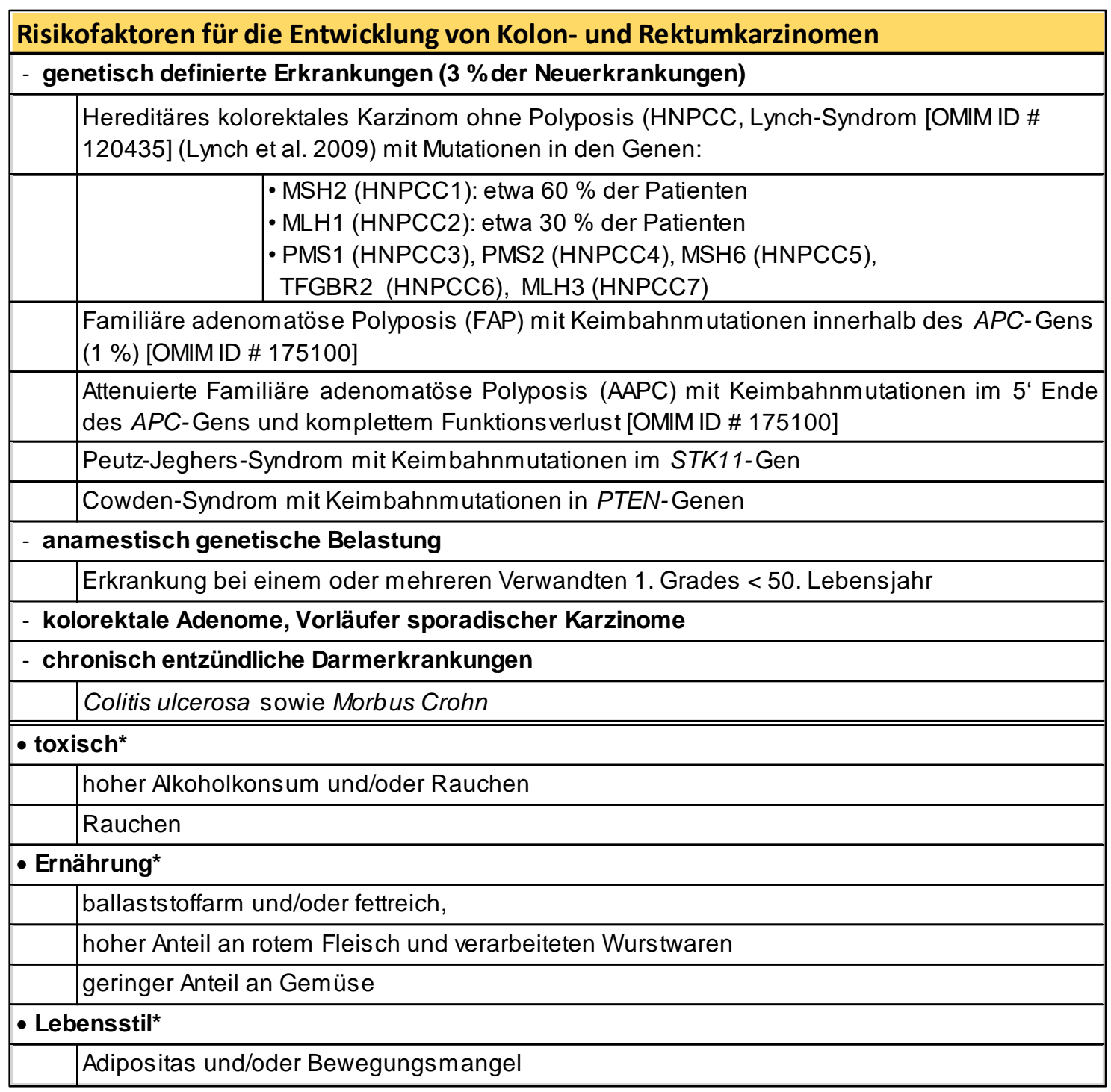

Tabelle A8: Risikofaktoren für das Auftreten von Kolon- und Rektumkarzinomen

*Aufgrund von unterschiedlichen Konzeptionen klinischer Studien sowie vielfältigen Kulturkreisen, Lebensformen und Selbstbeurteilungen der Studienteilnehmer haben die Ergebnisse zu den Ernährungs- und Lebensstil-assoziierten Risikofaktoren nicht dieselbe Wertigkeit wie die anderen genannten Risikofaktoren (nach Wörmann 2014).

\subsection{KRK - Karzinome unterschiedlicher Organsysteme}

Patienten mit HNPCC- und FAP-Syndromen entwickeln Karzinome überwiegend im rechten bzw. im linken Hemikolon. Das Ansprechen auf die jeweilige Tumorthe- 
rapie korreliert mit der Tumorlokalisation. Aufgrund klinischer Beobachtungen und molekularbiologischer Forschung können KRK heutzutage nicht mehr als eine Tumorentität angesehen werden (Holch et al. 2017). Es handelt sich insbesondere im Organsystem Kolon um molekulargenetisch/-pathologisch und prognostisch sehr unterschiedliche proximale oder distale sowie links- oder rechtsseitige Karzinomerkrankungen. Ebenso müssen die Adenokarzinome des oberen, mittleren und unteren Rektumdrittels molekularpathologisch vom Organsystem Kolon abgegrenzt werden (Holch et al. 2017; lacopetta 2002; Gervaz et al. 2001).

Mittlerweile beeinflussen die molekularpathologischen Erkenntnisse das therapeutische Vorgehen, wie es z. B. an der aktuellen sidedness-Debatte für das Kolonkarzinom im Stadium pUICC-IV zu erkennen ist. In diesem UICC-IV Stadium ist die infusionale Applikation von 5-FU + FS bzw. die orale Gabe von Capecitabin (Xeloda $\left.{ }^{\circledR}\right)$ Grundlage aller CTx-Regime. Beide Zytostatika werden wahlweise mit OX (FOLFOX-) und Irinotecan (FOLFIRI-) allein oder zusammen (FOLFOXIRIRegime) verabreicht. Zusätzlich zur CTx kann auf der Basis des RAS-GenMutationsstatus entweder der Einsatz von EGFR-Ak (z. B. Cetuximab, Panitumumab bei mKRK KRAS-Wildtyp) oder VEGFR-Ak (z. B. Bevacizumab, Ramucirumab, Afli-bercept bei Mutationsnachweis) erwogen werden. Zusätzlich zu dieser Standardempfehlung für eine CTx mit moAk (als Erstlinientherapie) kann eine weitere Stratifizierung der mKRK-Behandlung erfolgen (Arnold et al. 2017; AIO 2017; AIO 2016a; 2016 b).

Linksseitige Kolonkarzinome mit RAS-Wildtyp erhalten eine CTx mit EGFRInhibitoren während rechtsseitige Kolonkarzinome unabhängig vom RAS-Status einer kombinierten, Bevacizumab-haltigen Anti-VEGFR-Therapie zugeführt werden sollen. Eine Erweiterung der oralen CTx-Kombinationstherapie ist mit Trifluridin und Tipiracil (TAS-102) möglich (Mayer et al. 2015). Auch Immunmodulatoren, wie die Checkpoint-Inhibitoren (PD-1-Blocker) Nivolumab und Pembrolizumab, scheinen in der Therapie des Lynch-Syndroms bzw. bei Vorliegen einer MSI sinnvoll zu sein (Le et al. 2015). 


\subsection{Tumor-Klassifikation - Basissystematik}

Voraussetzung für die Umsetzung einer onkologisch effektiven Therapie ist eine umfassende klinische (c) Einschätzung des Tumorstadiums. Dieses ergibt sich beim Rektumkarzinom aus der Infiltrationstiefe des Tumors in die Rektumwand (T-Status), dem lokalen Nodal(N)-Status und einer ggf. vorliegenden Fernmetastasierung (M-Status) (Tabellen A9 und A10). Die Kategorisierung erfolgt nach der TNM-/UICC-Klassifikation des American Joint Committee of Cancer (AJCC,

TNM) und der Union Internationale Contre le Cancer (UICC) (Sobin et al. 2011).

\begin{tabular}{|c|c|l|}
\hline Kategorie & Code & \multicolumn{1}{|c|}{ Bedeutung } \\
\hline \multirow{4}{*}{ TNM-Klassifikation } & $\mathrm{T}$ & Primärtumor Ausdehnung \\
\cline { 2 - 3 } & $\mathrm{N}$ & regionärer Lymphknoten (vorhanden / fehlend) \\
\cline { 2 - 3 } & $\mathrm{M}$ & Fernmetastasen (vorhanden / fehlend) \\
\cline { 2 - 3 } & $\mathrm{C}$ & Befundsicherung durch Biopsie \\
\hline \multirow{4}{*}{ pTNM-Klassifikation } & $\mathrm{G}$ & Malignitätsgrading \\
\cline { 2 - 3 } & $\mathrm{L}$ & Lymphgefäßinvasion \\
\cline { 2 - 3 } & $\mathrm{V}$ & Veneninvasion \\
\cline { 2 - 3 } & $\mathrm{Pn}$ & Invasion der Perineuralscheiden \\
\cline { 2 - 3 } & $\mathrm{pT}$ & Primärtumor nach histologischer Untersuchung \\
\cline { 2 - 3 } & $\mathrm{pN}$ & regionärer Lymphknoten nach histologischer Untersuchung \\
\cline { 2 - 3 } & $\mathrm{pM}$ & Fernmetastasen nach histologischer Untersuchung \\
\hline R-Klassifikation & $\mathrm{R}$ & Residualtumore \\
\hline \multirow{3}{*}{$\begin{array}{c}\text { sonstige zusätzliche } \\
\text { Kennzeichen }\end{array}$} & $\mathrm{m}$ & multiple Tumore \\
\cline { 2 - 3 } & $\mathrm{r}$ & Rezidivtumor nach krankheitsfreiem Intervall \\
\cline { 2 - 3 } & $\mathrm{y}$ & Klassifikation nach initial erfolgter multimodaler Therapie \\
\cline { 2 - 3 } & $\mathrm{a}$ & Klassifikation bei Autopsie \\
\hline
\end{tabular}

Tabelle A9: Basissystematik der TNM-Klassifikation

Modifiziert nach 7. Auflage und mit Modifikation Wittekind und Meyer 2013

\subsubsection{Spezielle Aspekte der TNM-/UICC-Klassifikation}

Während der Laufzeit des Promotionsprojekts wurde die TNM-/UICC-Klassifikation mehrfach aktualisiert. Die TNM-/UICC-Klassifikation von 2010 (7. Auflage mit Modifikation von 2013) war Grundlage für die präsentierten Daten. In dieser 7. Auflage wurde erstmals eine Unterscheidung des M-Status eingeführt (Merkel et al. 2016; Wittekind und Meyer 2013) und in der gegenwärtigen 8. Auflage (gültig seit 01/2017) weiter subklassifiziert (z. B. M1c: Nachweis einer Peritonealkarzinose). 


\begin{tabular}{|c|c|}
\hline \multicolumn{2}{|r|}{ TNM Klassifikation } \\
\hline \multicolumn{2}{|c|}{ T - Primärtumor } \\
\hline T0 & kein Anhalt für Primärtumor \\
\hline Tis & Carcinoma in situ: intraepithelial oder Infiltration der Lamina propria \\
\hline T1 & Tumor infiltriert die Submukosa \\
\hline T2 & Tumor infiltriert Lamina muscularis propria (LMP) \\
\hline $\mathrm{T}^{* *}$ & $\begin{array}{l}\text { Tumor bricht } \underline{\text { durch }} \underline{\text { die }} \underline{\text { LMP }} \text { in die Subserosa oder in das (nicht peritonealisierte) perikolische oder } \\
\text { perirektale Gewebe }\end{array}$ \\
\hline- ТЗа & Infiltration $\leq 1 \mathrm{~mm}$ \\
\hline$-\mathrm{T3b}$ & Infiltration $>1-5 \mathrm{~mm}$ \\
\hline$-\mathrm{T} 3 \mathrm{c}$ & Infiltration > $5-15 \mathrm{~mm}$ \\
\hline$-\mathrm{T} 3 \mathrm{~d}$ & Infiltration > $15 \mathrm{~mm}$ \\
\hline T4 & Tumor infiltriert direkt in andere Organe oder Strukturen und/oder durchbricht das viszerale Peritoneum \\
\hline$-\mathrm{T} 4 \mathrm{a}$ & Tumorperforation in das viszerale Peritoneum \\
\hline$-\mathrm{T} 4 \mathrm{~b}$ & Tumorinfiltration in andere Organe oder Strukturen \\
\hline \multicolumn{2}{|c|}{ N - Lymphknoten } \\
\hline No & keine regionären Lymphknoten (Lk) - Metastasen \\
\hline N1 & Metastase $(n)$ in 1 bis 3 regionären Lk \\
\hline$-\mathrm{N} 1 \mathrm{a}$ & Metastase $(n)$ in 1 regionärem Lk \\
\hline$-\mathrm{N} 1 \mathrm{~b}$ & Metastase(n) in 2 bis 3 regionären Lk \\
\hline$-\mathrm{N} 1 \mathrm{c}$ & \begin{tabular}{|llllll}
$\begin{array}{l}\text { Karzinomknötchen bzw. Satellitenmetastasen* im Fettgewebe der Subserosa oder im nicht } \\
\text { peritonealisierten perikolischen/perirektalen (Fett-) Gewebe }\end{array}$ & & \\
\end{tabular} \\
\hline N2 & Metastasen in $\geq 4$ regionären Lk \\
\hline$-\mathrm{N} 2 \mathrm{a}$ & Metastasen in $4-6$ regionären Lk \\
\hline$-\mathrm{N} 2 \mathrm{~b}$ & Metastasen in $\geq 7$ regionären Lk \\
\hline \multicolumn{2}{|c|}{ M - Fernmetastasen } \\
\hline MO & keine Fernmetastasen \\
\hline M1 & Fernmetastasen \\
\hline$-\mathrm{M} 1 \mathrm{a}$ & Metastasen auf 1 Organ beschränkt (z. B. Leber, Lunge, Ovar) oder 1 Lokalisation \\
\hline$-\mathrm{M} 1 \mathrm{~b}$ & Metastasen in > 1 Organ oder Lokalisationen \\
\hline$-\mathrm{M} 1 \mathrm{c}$ & Peritonealbefall +/- Organmetastasen \\
\hline
\end{tabular}

Tabelle A10: Einteilung der KRK nach 8. Auflage der TNM-/UICC-Klassifikation

T: Tumor-Status, gibt die Tumor-Infiltrationstiefe in der Rektumwand und in das mesorektale Weichgewebe an; N: Nodal(N)-/Lymphknoten(LK)-Status weist auf eine Karzinominfiltration in lokoregionären LK hin oder auf Satellitenmetastasen ohne regionäre LK-Metastasen; M: M-Status beschreibt Fernmetastasen (mit Angabe des betroffenen Organs) (nach Wittekind und Meyer 2013); " als Satelliten werden makroskopisch oder mikroskopisch kleine Karzinomknötchen im Fettgewebe ohne histologisch erkennbare Reste eines LK angesehen;* die Subklassifikation der T3-Rektumkarzinome mit Infiltration jenseits der Lamina muscularis propria (LMP) entspricht der jeweiligen Tiefeninfiltration (in $\mathrm{mm}$ ) in das mesorektale/perirektale Weichgewebe. 
Grundlage für die Erweiterung der Klassifikation war u. a. eine Studie an 814 KRKPatienten im UICC-Stadium IV (Merkel et al. 2016). In dieser Studie hatte sich gezeigt, dass sowohl ein fortgeschrittenes Erkrankungsalter der Patienten als auch eine ubiquitäre Tumormetastasierung, eine inkomplette Tumorresektion wie auch frühzeitig einsetzende Therapiemaßnahmen voneinander unabhängige Prognosefaktoren waren. Patienten mit pulmonalen Metastasen hatten beispielsweise ein besseres OS gegenüber Patienten mit peritonealen und/oder lymphogenen FM. Patienten im M1a Stadium (d. h. FM in einem Organ, z. B. in der Leber oder in der Lunge) erreichten ein OS von zwei Jahren in 51,6 \% der Fälle. Patienten in einem M1b Stadium (FM in Leber und Lunge oder im Peritoneum oder in distanten Lymphknoten) hatten ein 2-Jahre OS nur in 39,4 \% der Fälle. Lag etwa ein M1c Status vor, d. h. waren Metastasen in einem anderen Organ (außer in der Leber und Lunge) oder in mehr als einem Organ mit peritonealem Befall nachweisbar, so erreichten nur 21,6 \% der Patienten ein 2-Jahre OS. Im klinischen Alltag wird beim UICC-IV Stadium die lokoregionäre, pelvine Tumorsituation nach erfolgter TME gesondert vom fernmetastastischen Status dokumentiert. D. h. bei synchron vorliegenden, nicht primär resektablen Lebermetastasen könnte ein RektumkarzinomBefund wie folgt lauten: pT2 pN0 (0/25 LK) M1 (hepar) R0 (lokal) R2 (hepar).

\begin{tabular}{|c|l|}
\hline UICC-Stadium & \multicolumn{1}{|c|}{ TNM-Klassifikation } \\
\hline I & T1 und T2, N0 M0 \\
\hline Ila & T3 N0 M0 \\
\hline Ilb & T4a N0 M0 \\
\hline Ilc & T4b N0 M0 \\
\hline Illa & T1 N2a und T1/2 N1a, M0 \\
\hline Illb & T1/2 N2b und T2/3 N2a und T3/4a N1, M0 \\
\hline Illc & T4b N1/2 und T3/4b N2b und T4a N2a, M0 \\
\hline IVa & jedes T und jedes N M1a \\
\hline IVb & jedes T und jedes N M1b \\
\hline IVc & jedes T und jedes N M1c \\
\hline
\end{tabular}

Tabelle A11: TNM-/UICC-Stadieneinteilung z. B. beim Rektumkarzinom Nach Wittekind 2017 


\subsubsection{Definition des postoperativen Residualstatus}

Die Einschätzung des Residualtumors $(\mathrm{R})$ beschreibt den Status der Malignomerkrankung nach abgeschlossener Behandlung bzw. erfolgter Rektumresektion (Tabelle A12). Der R-Status ist nicht mit dem Resektionsstatus gleich zu setzen.

\begin{tabular}{|c|l|}
\hline R-Klassifikation & \multicolumn{1}{|c|}{ Definition } \\
\hline RX & Vorhandensein von Residualtumor kann nicht beurteilt werden \\
\hline R0 & kein Residualtumor \\
\hline R1 & mikroskopisch nachgewiesener Residualtumor \\
\hline R2 & makroskopischer Residualtumor \\
\hline R2a & makroskopischer Residualtumor, mikroskopisch nicht bestätigt \\
\hline R2b & makroskopischer Residualtumor, mikroskopisch bestätigt \\
\hline
\end{tabular}

Tabelle A12: Residual(R)-Klassifikation

Nach Wittekind 2017

\subsubsection{Tumorzell-Grading}

In Anbetracht einer präoperativen MMT erfolgt das Malignitätsgrading beim Rektumkarzinom prätherapeutisch aus der diagnostischen Tumorbiopsie (Tabelle A13). In Zweifelsfällen wird immer das ungünstigere Grading berücksichtigt.

\begin{tabular}{|l|c|l|}
\hline Grading & Abkürzung & \multicolumn{1}{|c|}{ Graduierung der Malignität von Rektumkarzinomen } \\
\hline Grad x & Gx & Differenzierungsgrad nicht bestimmbar \\
\hline Grad 1 & G1 & $\begin{array}{l}\text { gut differenziertes malignes Gewebe (synonym: low-grade): } \\
\text { Die Zellen zeigen eine hohe Übereinstimmung mit dem } \\
\text { ursprünglichen Gewebe. }\end{array}$ \\
\hline Grad 2 & G2 & mäßig gut differenziertes malignes Gewebe \\
\hline Grad 3 & G3 & schlecht differenziertes malignes Gewebe \\
\hline Grad 4 & G4 & $\begin{array}{l}\text { undifferenziertes malignes Gewebe (synonym: high-grade): } \\
\text { Der Tumor zeigt keine Verwandtschaft zum ursprünglichen } \\
\text { Gewebe. Eine Zuordnung ist oft nur mittels } \\
\text { immunhistochemischer Spezialuntersuchungen möglich. }\end{array}$ \\
\hline
\end{tabular}

Tabelle A13: Tumorzell-Grading

Nach Wittekind 2017 


\subsubsection{Tumorregression-Grading nach präoperativer MMT}

In dieser Dissertation wurde folgendes Tumor-Regressionsgrading-System genutzt:

\begin{tabular}{|c|c|l|}
\hline $\begin{array}{c}\text { Tumor-Ansprechen auf } \\
\text { präoperative MMT }\end{array}$ & $\begin{array}{c}\text { Tumorregression } \\
\text { (TRG) }\end{array}$ & \multicolumn{1}{|c|}{ Kennzeichen } \\
\hline \multirow{2}{*}{ gutes Ansprechen (GR) } & TRG 4 & $\begin{array}{l}\text { keine Tumorzellen, nur fibrotische } \\
\text { Masse (totale Regression oder } \\
\text { Remission) }\end{array}$ \\
\cline { 2 - 3 } & TRG 3 & $\begin{array}{l}\text { sehr wenige (mikroskopisch schwer } \\
\text { zu findende) Tumorzellen im } \\
\text { fibrotischen Gewebe mit oder ohne } \\
\text { Schleimbildung }\end{array}$ \\
\hline \multirow{2}{*}{ partielles Ansprechen (PR) } & TRG 2 & $\begin{array}{l}\text { vorwiegend fibrotische Veränderung } \\
\text { mit wenigen Tumorzellen oder } \\
\text {-gruppen (leicht zu finden) }\end{array}$ \\
\cline { 2 - 3 } & TRG 1 & $\begin{array}{l}\text { dominante Tumormasse mit } \\
\text { offensichtlicher Fibrose und/oder } \\
\text { Vaskulopathie }\end{array}$ \\
\hline kein Ansprechen (Resistenz) & TRG 0 & \begin{tabular}{l} 
keine Regression \\
\hline
\end{tabular}
\end{tabular}

Tabelle A14: Tumor-Ansprechen auf eine präoperative Multimodaltherapie gruppiert nach TRG

Modifiziert nach Song et al. 2018 und Dworak et al. 1997

\subsection{Prinzipien der MMT und Chirurgie beim Rektumkarzinom}

Unabhängig von den präoperativen Maßnahmen stellt die onkologische Resektion nach wie vor die Therapie der Wahl beim lokal fortgeschrittenen Rektumkarzinom dar. Dabei besteht das Ziel der Rektumresektion im Erreichen eines histopathologisch bestätigten R0-Status (Compton 2002). Die während der Laufzeit des Promotionsprojekts durchgeführten onkologischen Operationen umfassten (Becker et al. 2012; Liersch et al. 2007a; 2007b) die kontinenzerhaltende, tiefe anteriore Rektumresektion (TARR) mit totaler mesorektaler Exzision (TME) bei Rektumkarzinomen, die $>1 \mathrm{~cm}$ oberhalb des Schließmuskeloberrandes bis zu $12 \mathrm{~cm}$ ab Anokutanlinie lagen und die kontinenzerhaltende, ultratiefe anteriore Rektumresektion (utTARR) unter Teilresektion des Schließmuskelapparates und mit Anlage einer koloanalen Anastomose (Cavaliere et al. 1995) bei Rektumkarzinomen, die entweder $<1 \mathrm{~cm}$ oberhalb des Schließmuskeloberrandes lagen oder diesen infiltrier- 
ten. Ebenso wurde die kontinenzunterbrechende Operation nach Hartmann (Sanderson 1980) mit TME unter Erhalt des Schließmuskelapparates und der Beckenbodenmuskulatur mit Anlage eines endständigen Kolostomas angewandt. Dieses OP-Verfahren ermöglichte bei intakter Schließmuskelfunktion eine spätere, die Kontinenz wiederherstellende Descendorektostomie. Bei bestehender muskulärer Sphinkter- und Beckenbodeninsuffizienz verblieb das endständige Kolostoma und von einer chirurgischen Kontinenzwiederherstellung wurde Abstand genommen (S3-Leitlinie Kolorektales Karzinom 2019; Perry und Connaughton 2007). Bei Tumoren, die im unteren Rektumdrittel lokalisiert waren und großflächig den Sphinkter infiltrierten wurde eine abdominoperineale Rektumexstirpation (APE) mit TME durchgeführt (Heald et al. 1997; Smith et al. 1988). Die APE umfasste die Resektion des Schließmuskelapparates mit partieller oder kompletter Resektion der Levatormuskulatur und des Beckenbodens sowie ggf. ossärer Nachbarstrukturen (z. B. Steißbeinresektion) (West et al. 2010; 2008; Holm et al. 2007; Nagtegaal et al. 2005).

Alle transabdominellen OP-Verfahren, unabhängig davon, ob diese laparoskopisch assistiert oder konventionell offen (als mediane Laparotomie) durchgeführt wurden, waren onkologisch radikale Resektionsverfahren. Sie erfolgten als en-bloc Resektion des tumortragenden Kolon-/Rektumabschnitts und des Lymphabstromgebietes (Schmiegel et al. 2008; Nagtegaal et al. 2005; Martling et al. 2002).

\subsubsection{Rektumresektat-Beurteilung durch Chirurgen}

Die Rektumresektionen erfolgten unter Einhaltung qualitätssichernder Maßnahmen. Diese umfassten eine perioperative Überprüfung der TME-Qualität unmittelbar nach der Bergung des Resektates aus dem Situs durch einen vom OP-Team unabhängigen Chirurgen nach den Kategorien komplette, moderate oder inkomplette Mesorektumexzision (Liersch et al. 2009). Bei der kompletten Mesorektumexzision war das Mesorektum intakt, mit nur geringen Unregelmäßigkeiten der glatten, lipomähnlichen Mesorektumoberfläche (intakte Hülfaszie), kein Defekt 
war > $5 \mathrm{~mm}$; bei der queren Lamellierung des Resektats zur Darmlängsachse zeigte sich ein glatter zirkumferentieller Resektionsrand (CRM). Die moderate Mesorektumexzision zeigte eine unregelmäßige Oberfläche des Mesorektums; an keiner Stelle war die LMP sichtbar (außer am Ansatz der Levatormuskulatur); bei querer Lamellierung bestand eine mäßiggradige Unregelmäßigkeit am CRM. In der Kategorie inkomplette Mesorektumexzision lagen nur geringe Mengen an Mesorektum vor; an der Oberfläche waren Defekte bis zur LMP nachweisbar; bei querer Lamellierung zeigte sich ein sehr unregelmäßiger CRM.

\subsubsection{Rektumresektat-Beurteilung durch Pathologen}

Im Anschluss an die perioperative Beurteilung wurde das Rektumresektat nach Faden-Markierungen in allen Resektionsebenen an das ärztliche Befundungsteam des Instituts für Pathologie an der UMG weitergeleitet. In der PräparateAnnahmestelle wurde das Ausmaß der Mesorektumentfernung dokumentiert, makroskopisch die TME-Resektatoberfläche beurteilt, fotodokumentiert und anschließend das Rektumresektat für die weiteren histopathologischen Begutachtungen aufgearbeitet. Im Fall einer erweiterten (extralevatorischen) APE wurden die resezierten Anteile der Beckenbodenmuskulatur (M. levator ani) ebenso dokumentiert, wie die Komplettheit des entfernten Sphinkterapparates und ggf. der Nachbarorgane (z. B. von Prostata, Samenblasen, Harnblasenwand oder der vaginalen Hinterwand). Zur Befunderhebung am Rektumresektat: umfasste nach Liersch et al. (2007a; 2006b) die Resektataufarbeitung das makroskopische Ausmessen des Abstands zwischen dem Tumorrand (oral, aboral, lateral) und der Resektionsebene am frischen Resektat ohne Zug und Ausspannen (Goldstein et al. 1999) sowie das Vermessen der Präparatlänge mit Angabe der longitudinalen und transversalen Tumorausdehnung; Beschreibung der Wuchsformen: polypoidgestielt, -tailliert, -sessil; plattenartig, ulzerös, szirrhös (Hermanek et al. 2003; Hermanek 1999a). Weiterhin erfolgte die Dokumentation der Lage des Tumors zur peritonealen Umschlagsfalte mit assoziierten Läsionen (z. B. Polypen). Durch die Eröffnung des Präparates oberhalb und unterhalb des Tumors wurde sicherge- 
stellt, dass das tumortragende Darmsegment und der Tumor nicht durchschnitten wurden. Zur Resektataufarbeitung gehörten außerdem das Einbringen einer von endoluminal stabilisierenden Tamponade in die Tumorregion mitsamt den angrenzenden distalen und proximalen Darmabschnitten, ein zugfreies Aufspannen des Resektates und eine Formalinfixierung über 48 bis 72 h, die Anfärbung der Mesorektumoberfläche nach Fixation und Beurteilung sowie lamellierende Querschnitte durch das tumortragende Darmsegment und das umgebende Mesorektum mit Einbettung relevanter Präparatescheiben zur Beurteilung der perirektalen Karzinominfiltration und der minimalen Entfernung des Tumors zum CRM (Hermanek et al 2015; 2003; Liersch et al. 2006a; Hermanek 1999b).

Sämtliche Resektataufarbeitungen mit den anschließenden histopathologischen Untersuchungen wurden von erfahrenen Pathologen (initial: Frau OÄ Dr. med. H. Rothe; später: Frau OÄ Dr. med. J. Kitz) und weiteren Mitarbeitern im Institut für Pathologie der UMG durchgeführt.

Die Basisdokumentation beinhaltete Angaben zur Tumorklassifikation nach dem TNM-/UICC-System (Schmiegel et al. 2008) und beschreibt die Lokalisation des Tumors im Enddarm, den Tumortyp nach WHO-Klassifikation sowie die ausgemessene Tumorinvasionstiefe (pT-Status). Die Festlegung des regionären Lymphknotenstatus (pN-Status), wobei > 12 LK im Resektat untersucht sein mussten (empfohlen waren $\geq 12$ bis 17 untersuchte LK im Rektumresektat) (Scheel et al. 2015; Gehoff et al. 2012; Hida et al. 1997) und die Anzahl der LK-Metastasen mit morphologischer Beschreibung waren ebenfalls Bestandteil der Dokumentation. Sowohl der Differenzierungsgrad der Karzinomzellen (G1 bis G4) als auch der Residualstatus (R0-, R1- und R2-Einteilung) mit Darstellung einer Lymphgefäßinvasion (L1) oder Blutgefäßinvasion (V1) wurden dokumentiert. Die tumorfreien Resektionsränder (oral, aboral und lateral) wurden mikroskopisch und immunhistochemisch untersucht, der karzinomfreie CRM in $\mathrm{mm}$ gemessen, wobei ein CRM $\leq 1 \mathrm{~mm}$ als CRM-positiv bewertet wurde. In Fällen, in denen präoperativ eine neoadjuvante RCT oder CTx durchgeführt worden war, wurde die Festlegung der Tumorregression (TRG) nach Dworak et al. (1997) getroffen. Die Qualitätsbeurteilung der Mesorektumresektion erfolgte nach MERCURY-Kriterien. 


\subsection{Fachinformationen zu 5-FU, FS und OX}

Die nachfolgenden Informationen zu den in den Studien verwendeten CTx Medikationen wurden am 07.01.2020 über die angegebenen Links eingesehen.

\section{5-FU}

https://imedikament.de/fluorouracil-hexal-50-mg-ml/fachinformation

Handelsname: $\quad$ Fluorouracil HEXAL® $50 \mathrm{mg} / \mathrm{ml}$ Injektionslösung

Zulassungsnummer: $\quad 84261.00 .00$

Inhaber der Zulassung: Hexal AG

Stand der Information: Februar 2014

\section{Folinsäure (FS)}

http://fachinformation.srz.de/pdf/pfizerpfe/leucovorin10mgmll\%C3\%B6sung.pdf

Handelsname: $\quad$ Leucovorin $10 \mathrm{mg} / \mathrm{ml}$ Lösung zur Injektion/Infusion

Zulassungsnummer: $\quad 15034.00 .00$

Inhaber der Zulassung: Pfizer Pharma PEE GmbH

Stand der Information: März 2019

\section{Oxaliplatin (OX)}

https://mein.sanofi.de/produkte/Eloxatin/Downloads?id=dafcc9b3-4397-4205aeb3-581a3fa90edd

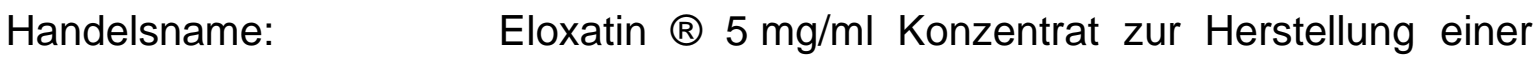
Infusionslösung

Zulassungsnummer: $\quad 63264.00 .00$

Inhaber der Zulassung: Sanofi-Aventis Deutschland GmbH

Stand der Information: Februar 2019 


\subsection{Therapiepläne der Kohorten A, B und C}

Die nachfolgenden Abbildungen zeigen Muster der verwendeten Therapiepläne. Die enthaltenen Daten zu Gewicht, Größe und Therapietagen des ersten CTx-Zyklus dienen nur zur Veranschaulichung und haben keine Übereinstimmung mit den in dieser Dissertation genutzten Patientendaten.

Ergänzung zur supportiven Therapie: Der Granulozyten-Kolonie-stimulierende Faktor (G-CSF) musste bei keinem Patienten im Therapieverlauf eingesetzt werden, da den auftretenden Leukopenien/Neutropenien durch rechtzeitige Dosisreduktionen der CTx bzw. durch Therapiepausen adäquat begegnet werden konnte. 


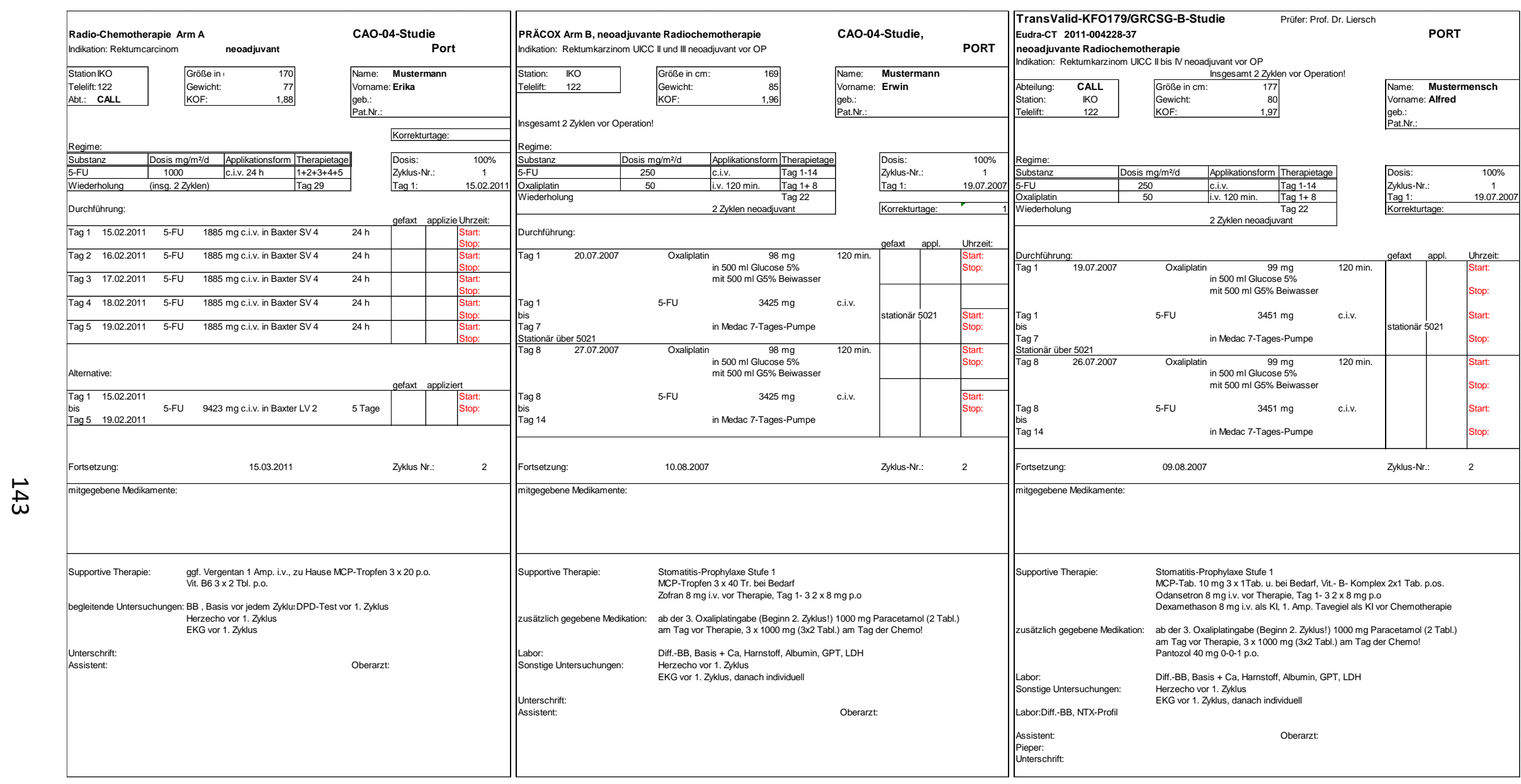

Abbildung A2: Therapieplan der Kohorten A, B und C für präoperative CTx 


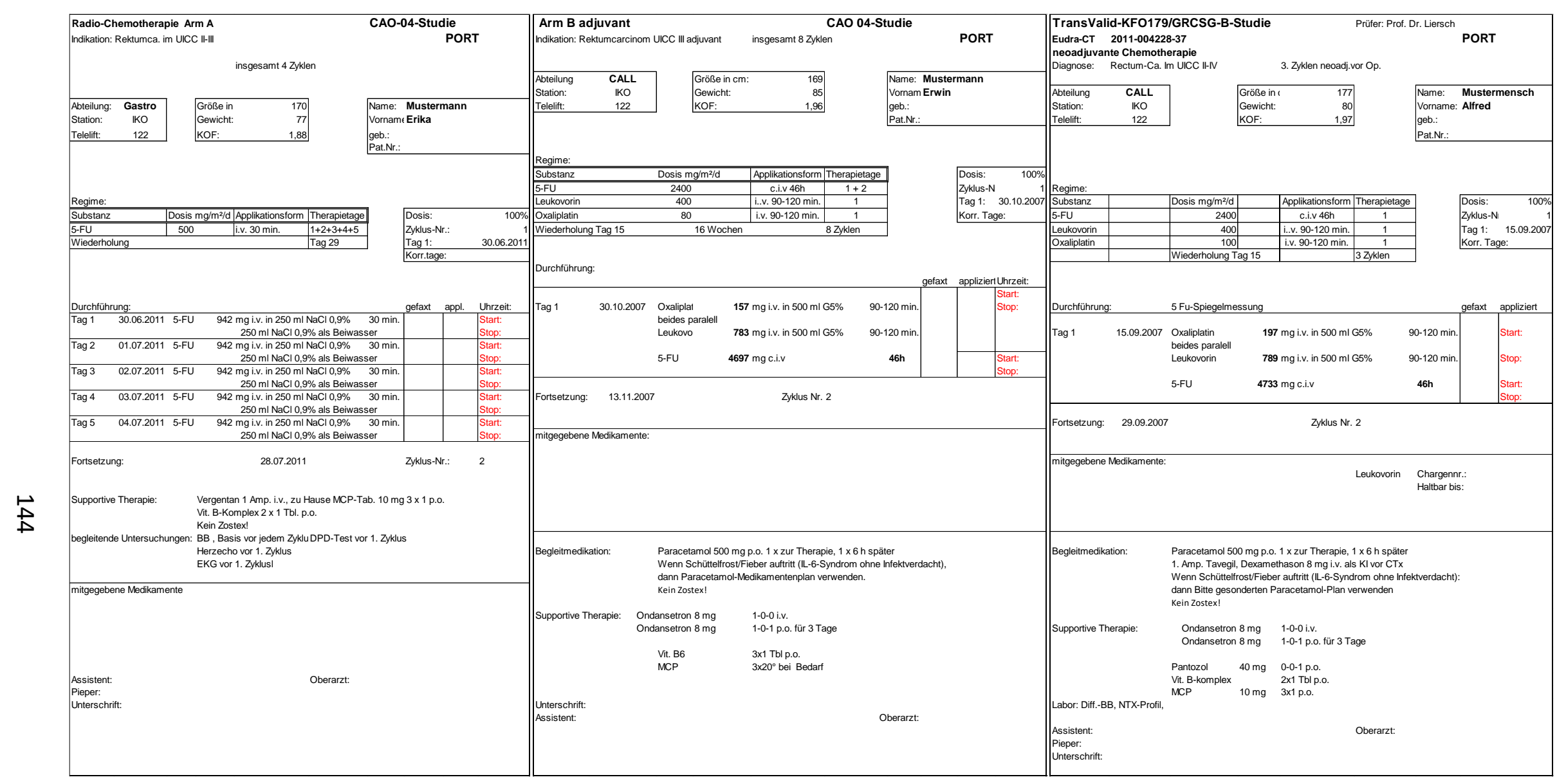

Abbildung A3: Therapiepläne der Kohorten A und B für postoperative CTx, Kohorte C präoperative CTx nach CRT 


\section{$7 \quad$ Literaturverzeichnis}

Aaltonen LA (1998): Molecular epidemiology of hereditary nonpolyposis colorectal cancer in Finland. Recent Results Cancer Res 154, 306-311

Adjei AA, Reid JM, Diasio RB, Sloan JA, Smith DA, Rubin J, Pitot HC, Alberts SR, Goldberg RM, Hanson LJ et al. (2002): Comparative pharmacokinetic study of continuous venous infusion fluorouracil and oral fluorouracil with eniluracil in patients with advanced solid tumors. J Clin Oncol 20, 1683-1691

Agarwal A, Chang GJ, Hu CY, Taggart M, Rashid A, Park IJ, You YN, Das P, Krishnan S, Crane CH et al. (2013): Quantified pathologic response assessed as residual tumor burden is a predictor of recurrence-free survival in patients with rectal cancer who undergo resection after neoadjuvant chemoradiotherapy. Cancer $\underline{119}, 4231-4241$

A'Hern RP (2016): Restricted mean survival time: an obligatory endpoint for time-to-event analysis in cancer trials? J Clin Oncol $\underline{34}$, 3474-3476

AIO (2016a): Statement der AIO-KRK-Leitgruppe zur Wahl der Erstlinientherapie bei Patienten mit metastasiertem Kolorektalkarzinom ohne Nachweis einer RAS Mutation unter Berücksichtigung der primären Tumorlokalisation: Update 31. Oktober: Zusammenfassung der aktuellen Datenlage. https://www.aioportal.de/tl_files/aio/stellungnahmen/Statement\%20der\%20AIO_update\%20Oktober\%202016.pdf; abgerufen am: $13.01 . \overline{2} 020$

AIO (2016b): AIO-Jahresbericht: Kolon-/Rektum-/Dünndarmkarzinom. Seiten 17-18. https://www.aioportal.de/tl_files/aio/aktuell/Mitgliederrundschreiben\%202016\%20NEU.pdf; abgerufen am: 13.01.2020

AIO, Arbeitsgemeinschaft Internistische Onkologie in der Deutschen Krebsgesellschaft e.V. (AIO) (2017): Statement der AIO-KRK Leitgruppe zur adjuvanten Therapie im Stadium III (IDEA-Studien). https://www.aioportal.de/tl_files/aio/stellungnahmen/Statement\%20der\%20AIO-KRK\%20Leitgruppe_14_06_2017.pdf; abgerufen am: 13.01.2020

Aktories K, Forth W, Allgaier C (Hrsg.): Allgemeine und spezielle Pharmakologie und Toxikologie: Für Studenten der Medizin, Veterinärmedizin, Pharmazie, Chemie und Biologie sowie für Ärzte, Tierärzte und Apotheker; mit 305 Tabellen. 10. Auflage; Elsevier Urban \& Fischer, München 2010

Amin MB, Greene FL, Edge SB, Compton CC, Gershenwald JE, Brookland RK, Meyer L, Gress DM, Byrd DR, Winchester DP (2017): The eighth edition AJCC cancer staging manual: continuing to build a bridge from a population-based to a more "personalized" approach to cancer staging. CA Cancer J Clin 67, 93-99

André T, Boni C, Mounedji-Boudiaf L, Navarro M, Tabernero J, Hickish T, Topham C, Zaninelli M, Clingan P, Bridgewater $\mathrm{J}$ et al. (2004): Oxaliplatin, fluorouracil, and leucovorin as adjuvant treatment for colon cancer. $\mathrm{N}$ Engl J Med 350, 2343-2351

André T, Boni C, Navarro M, Tabernero J, Hickish T, Topham C, Bonetti A, Clingan P, Bridgewater J, Rivera F et al. (2009): Improved overall survival with oxaliplatin, fluorouracil, and leucovorin as adjuvant treatment in stage II or III colon cancer in the MOSAIC trial. J Clin Oncol 27, 3109-3116

André T, Iveson T, Labianca R, Meyerhardt JA, Souglakos I, Yoshino T, Paul J, Sobrero A, Taieb J, Shields AF et al. (2013): The IDEA (International Duration Evaluation of Adjuvant Chemotherapy) Collaboration: prospective combined analysis of phase III trials investigating duration of adjuvant therapy with the FOLFOX (FOLFOX4 or Modified FOLFOX6) or XELOX (3 versus 6 months) regimen for patients with stage III colon cancer: Trial design and current status. Curr colorectal cancer Rep $\underline{9}, 261-269$

Arellano M, Malet-Martino M, Martino R, Gires P (1998): The anti-cancer drug 5-fluorouracil is metabolized by the isolated perfused rat liver and in rats into highly toxic fluoroacetate. Br J Cancer $\underline{77}, 79-86$

Arnold D, Lueza B, Douillard JY, Peeters M, Lenz HJ, Venook A, Heinemann V, van Cutsem E, Pignon JP, Tabernero J et al. (2017): Prognostic and predictive value of primary tumour side in patients with RAS wildtype metastatic colorectal cancer treated with chemotherapy and EGFR directed antibodies in six randomized trials. Ann Oncol $\underline{28}, 1713-1729$

Babaei M, Balavarca Y, Jansen L, Gondos A, Lemmens V, Sjövall A, Brge Johannesen T, Moreau M, Gabriel L, Gonçalves AF et al. (2016): Minimally invasive colorectal cancer surgery in europe: implementation and outcomes. Medicine (Baltimore) $\underline{95}$, e3812 
Barillari P, Ramacciato G, de Angelis R, Gozzo P, Indinnimeo M, Valabrega S, Aurello P, Fegiz G (1990): Effect of preoperative colonoscopy on the incidence of synchronous and metachronous neoplasms. Acta Chir Scand $\underline{156}, 163-166$

Becker H, Homayounfar K, Ghandimi M (Hrsg.): Open total mesorectal excision (TME) for rectal cancer (Operation Primer. Band 10). 1. Auflage; Springer, Stuttgart 2012

Becker H, Liersch T: Kolonkarzinom, Rektumkarzinom. In: Becker H, Encke A, Röhr HD (Hrsg.): Viszeralchirurgie. 2. Auflage; Elsevier Urban \& Fischer, München 2006, 579-631

Beets-Tan RG, Beets GL, Vliegen RF, Kessels AG, van Boven H, de Bruine A, von Meyenfeldt MF, Baeten CG, van Engelshoven JM (2001): Accuracy of magnetic resonance imaging in prediction of tumour-free resection margin in rectal cancer surgery. Lancet $\underline{357}$, 497-504

Beets-Tan RGH, Beets GL (2004): Rectal cancer: review with emphasis on MR imaging. Radiology 232, 335346

Beets-Tan RGH, Lambregts DMJ, Maas M, Bipat S, Barbaro B, Curvo-Semedo L, Fenlon HM, Gollub MJ, Gourtsoyianni S, Halligan S et al. (2018): Magnetic resonance imaging for clinical management of rectal cancer: updated recommendations from the 2016 european society of gastrointestinal and abdominal radiology (ESGAR) consensus meeting. Eur Radiol 28, 1465-1475

Bertelsen CA, Neuenschwander AU, Jansen JE, Wilhelmsen M, Kirkegaard-Klitbo A, Tenma JR, Bols B, Ingeholm P, Rasmussen LA, Jepsen LV et al. (2015): Disease-free survival after complete mesocolic excision compared with conventional colon cancer surgery: a retrospective, population-based study. Lancet Oncol $\underline{16}$, 161-168

Biagi JJ, Raphael MJ, Mackillop WJ, Kong W, King WD, Booth CM (2011): Association between time to initiation of adjuvant chemotherapy and survival in colorectal cancer: a systematic review and meta-analysis. JAMA $305,2335-2342$

Birgisson H, Wallin U, Holmberg L, Glimelius B (2011): Survival endpoints in colorectal cancer and the effect of second primary other cancer on disease free survival. BMC Cancer $\underline{11}, 438$

BMG (2018): Beschluss des Gemeinsamen Bundesausschusses über eine Richtlinie für organisierte Krebsfrüherkennungsprogramme und eine Änderung der Krebsfrüherkennungs-Richtlinie; In: Bundesanzeiger $\underline{2018}$, 1-25;

https://www.bundesanzeiger.de/ebanzwww/contentloader?state.action=genericsearch loadpublicationpdf\&session.sessioni

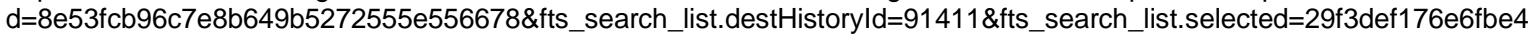
\&state.filename=BAnz\%20AT\%2018.10.2018\%20B3; abgerufen am 31.01.2020

Boisdron-Celle M, Remaud G, Traore S, Poirier, AL, Morel, A, Gamelin E (2007): 5-fluorouracil-related severe toxicity: a comparison of different methods for the pretherapeutic detection of dihydropyrimidine dehydrogenase deficiency. Cancer Letters $\underline{249}, 271-282$

Bonnetain F, Borg C, Adams RR, Ajani JA, Benson A, Bleiberg H, Chibaudel B, Diaz-Rubio E, Douillard JY, Fuchs CS et al. (2017): How health-related quality of life assessment should be used in advanced colorectal cancer clinical trials. Ann Oncol 28, 2077-2085

Bosset JF, Collette L, Calais G, Mineur L, Maingon P, Radosevic-Jelic L, Daban A, Bardet E, Beny A, Ollier JC (2006): Chemotherapy with preoperative radiotherapy in rectal cancer. N Engl J Med $\underline{355}, 1114-1123$

Bosset JF, Calais G, Mineur L, Maingon P, Stojanovic-Rundic S, Bensadoun RJ, Bardet E, Beny A, Ollier JC, Bolla $\mathrm{M}$ et al. (2014): Fluorouracil-based adjuvant chemotherapy after preoperative chemoradiotherapy in rectal cancer: long-term results of the EORTC 22921 randomised study. Lancet Oncol 15, 184-190

Bray F, Ferlay J, Soerjomataram I, Siegel RL, Torre LA, Jemal A (2018): Global cancer statistics 2018: GLOBOCAN estimates of incidence and mortality worldwide for 36 cancers in 185 countries. CA Cancer J Clin $\underline{68}$ 394-424

Brenner H, Kloor M, Pox CP (2014a): Colorectal cancer. The Lancet $\underline{383}$, 1490-1502

Brenner H, Stock C, Hoffmeister M (2014b): Effect of screening sigmoidoscopy and screening colonoscopy on colorectal cancer incidence and mortality: systematic review and meta-analysis of randomised controlled trials and observational studies. BMJ $\underline{348}, \mathrm{~g} 2467$

Brenner H, Schrotz-King P, Holleczek B, Katalinic A, Hoffmeister M (2016): Declining bowel cancer incidence and mortality in Germany. Dtsch Arztebl Int 113, 101-106

Brenner H, Zwink N, Ludwig L, Hoffmeister M (2017): Should screening colonoscopy be offered from age 50 ? Dtsch Arztebl Int 114, 94-100 
Breugom AJ, van Gijn W, Muller EW, Berglund Å, van den Broek CBM, Fokstuen T, Gelderblom H, Kapiteijn E, Leer JWH, Marijnen CAM et al. (2015a): Adjuvant chemotherapy for rectal cancer patients treated with preoperative (chemo)radiotherapy and total mesorectal excision: a Dutch Colorectal Cancer Group (DCCG) randomized phase III trial. Ann Oncol 26 6 , 696-701

Breugom AJ, Swets M, Bosset JF, Collette L, Sainato A, Cionini L, Glynne-Jones R, Counsell N, Bastiaannet E, van den Broek CBM et al. (2015b): Adjuvant chemotherapy after preoperative (chemo)radiotherapy and surgery for patients with rectal cancer: a systematic review and meta-analysis of individual patient data. Lancet Oncol 16, 200-207

Bruck MA, Bau R, Noji M, Inagaki K, Kidani Y (1984): The crystal structures and absolute configurations of the anti-tumor complexes $\mathrm{Pt}$ (oxalato)(1R,2R-cyclohexanediamine) and $\mathrm{Pt}$ (malonato)(1R,2R-cyclohexanediamine). Inorganica Chimica Acta $\underline{92}, 279-284$

Buyse M, Molenberghs G, Paoletti X, Oba K, Alonso A, van der Elst W, Burzykowski T (2016): Statistical evaluation of surrogate endpoints with examples from cancer clinical trials. Biom J $\underline{58}, 104-132$

Capitain O, Boisdron-Celle M, Poirier AL, Abadie-Lacourtoisie S, Morel A, Gamelin E (2008): The influence of fluorouracil outcome parameters on tolerance and efficacy in patients with advanced colorectal cancer. Pharmacogenomics $\mathrm{J} \underline{8}, 256-267$

Carvalho C, Glynne-Jones R (2017): Challenges behind proving efficacy of adjuvant chemotherapy after preoperative chemoradiation for rectal cancer. Lancet Oncol 18, e354-e363

Cavaliere F, Pemberton JH, Cosimelli M, Fazio VW, Beart RW (1995): Coloanal anastomosis for rectal cancer. Long-term results at the Mayo and Cleveland Clinics. Dis Colon Rectum $\underline{38}, 807-812$

Chand M, Swift RI, Chau I, Heald RJ, Tekkis PP, Brown G (2014): Adjuvant therapy decisions based on magnetic resonance imaging of extramural venous invasion and other prognostic factors in colorectal cancer. Ann R Coll Surg Engl 96, 543-546

Chaney S (1995): The chemistry and biology of platinum complexes with the 1,2-diaminocyclohexane carrier ligand (review). Int J Oncol $\underline{6}, 1291-1305$

Chang GJ (2018): Is there validity in propensity score-matched estimates of adjuvant chemotherapy effects for patients with rectal cancer? JAMA Oncol $\underline{4}$, 921-923

Chen C, Stock C, Hoffmeister M, Brenner H (2018): Public health impact of colonoscopy use on colorectal cancer mortality in Germany and the United States. Gastrointest Endosc 87, 213-221

Cheung WY, Neville BA, Earle CC (2009): Etiology of delays in the initiation of adjuvant chemotherapy and their impact on outcomes for stage II and III rectal cancer. Dis Colon Rectum 52, 1054-1064

Collette L, Bosset JF, den Dulk M, Nguyen F, Mineur L, Maingon P, Radosevic-Jelic L, Piérart M, Calais G (2007): Patients with curative resection of cT3-4 rectal cancer after preoperative radiotherapy or radiochemotherapy: does anybody benefit from adjuvant fluorouracil-based chemotherapy? A trial of the european organisation for research and treatment of cancer radiation oncology group. J Clin Oncol $\underline{25}$, 4379-4386

Compton CC (2002): Pathologic prognostic factors in the recurrence of rectal cancer. Clin Colorectal Cancer 2, $149-160$

Cox DR (1972): Regression models and life-tables. J Roy Statist Soc Series B (Methodological) 34 (2), $187-$ 220

DGHO (2013): Stellungnahme zur Nutzenbewertung gemäß § 35a SGB V Aflibercept.

https://www.dgho.de/publikationen/stellungnahmen/fruehe-nutzenbewertung/aflibercept/aflibercept-dghostellungnahme-20130624.pdf; abgerufen am: 12.01.2020

DGHO (2019): Deutschlandweite Prognose der bevölkerungsbezogenen Morbiditätserwartung für häufige Krebserkrankungen: Auswirkungen auf die Versorgung (Gesundheitspolitische Schriftenreihe der DGHO 14); DGHO, Berlin 2019

Di Francesco AM, Ruggiero A, Riccardi R (2002): Cellular and molecular aspects of drugs of the future: oxaliplatin. Cell Mol Life Sci $\underline{59}, 1914-1927$ 
Díaz Rubio E, Tabernero J, van Cutsem E, Cervantes A, André T, Humblet Y, Soulié P, Corretgé S, Kisker O, Gramont A de (2005): Cetuximab in combination with oxaliplatin/5-fluorouracil (5-FU)/folinic acid (FA) (FOLFOX-4) in the first-line treatment of patients with epidermal growth factor receptor (EGFR)-expressing metastatic colorectal cancer: an international phase II study. J Clin Oncol $\underline{23}, 3535$

DIMDI Deutsches Institut für Medizinsche Dokumentation und Information (DIMDI) im Auftrag des Bundesministerium für Gesundheit (BMG) unter Beteiligung der Arbeitsgruppe ICD des Kuratoriums für Fragen der Klassifikation im Gesundheitswesen (KKG) (2019): ICD-10-GM Version 2019, Systematisches Verzeichnis, Internationale statistische Klassifikation der Krankheiten und verwandter Gesundheitsprobleme, 10. Revision, Stand: 21.September 2018: Köln 2018 S. 75, Köln. www.dimidi.de - Klassifikation - Downloads - ICD-10-GM Version 2019; abgerufen am 13.01.2020

Dossa F, Acuna SA, Rickles AS, Berho M, Wexner SD, Quereshy FA, Baxter NN, Chadi SA (2018): Association between adjuvant chemotherapy and overall survival in patients with rectal cancer and pathological complete response after neoadjuvant chemotherapy and resection. JAMA Oncol $\underline{4}, 930-937$

Du Bois D, Du Bois EF (1989): A formula to estimate the approximate surface area if height and weight be known. 1916. Nutrition $\underline{5}, 303-313$

Duffy MJ (2001): Carcinoembryonic antigen as a marker for colorectal cancer: is it clinically useful? Clin Chem $\underline{47}, 624-630$

Dworak O, Keilholz L, Hoffmann A (1997): Pathological features of rectal cancer after preoperative radiochemotherapy. Int J Colorectal Dis $\underline{12}, 19-23$

Elliort K, Birch J: Symposium on carbon-fluorine compounds: chemistry, biochemistry and biological activities (Ciba Foundation symposium). Associated Scientific Publishers, Amsterdam, New York 1972

Elliot AH, Martling A, Glimelius B, Johansson H, Nilsson PJ (2016): Impact of pre-treatment patient-related selection parameters on outcome in rectal cancer. Eur J Surg Oncol 42, 1667-1673

Enker WE (1997): Total mesorectal excision - the new golden standard of surgery for rectal cancer. Ann Med $\underline{29}, 127-133$

Extermann M, Bonetti M, Sledge GW, O'Dwyer PJ, Bonomi P, Benson AB (2004): MAX2 - a convenient index to estimate the average per patient risk for chemotherapy toxicity; validation in ECOG trials. Eur J Cancer $\underline{40}$, 1193-1198

Fang F, Hoskins J, Butler JS (2004): 5-fluorouracil enhances exosome-dependent accumulation of polyadenylated rRNAs. Mol Cell Biol 24, 10766-10776

Fearon ER, Vogelstein B (1990): A genetic model for colorectal tumorigenesis. Cell $\underline{61}$, 759-767

Feinstein AR, Sosin DM, Wells CK (1985): The Will Rogers phenomenon. Stage migration and new diagnostic techniques as a source of misleading statistics for survival in cancer. N Engl J Med 312, 1604-1608

Fibbe C: Sequenzielle Therapie beim kolorektalen Karzinom - eine Orientierung für den Nicht-Onkologen. In: Arzneimittelkommission der deutschen Ärzteschaft (Hrsg.): Arzneiverordnung in der Praxis Jahrgang 45 Ausgabe 1, Berlin 2018, 13-22

https://www.akdae.de/Arzneimitteltherapie/AVP/Ausgaben/ab2015/201801.pdf; abgerufen am 12.01.2020

Floriani I, Torri V, Rulli E, Garavaglia D, Compagnoni A, Salvolini L, Giovagnoni A (2010): Performance of imaging modalities in diagnosis of liver metastases from colorectal cancer: a systematic review and metaanalysis. J Magn Reson Imaging 31, 19-31

Fokas E, Liersch T, Fietkau R, Hohenberger W, Beissbarth T, Hess C, Becker H, Ghadimi M, Mrak K, Merkel $S$ et al. (2014): Tumor regression grading after preoperative chemoradiotherapy for locally advanced rectal carcinoma revisited: updated results of the CAO/ARO/AIO-94 trial. J Clin Oncol $\underline{32}, 1554-1562$

Fokas E, Liersch T, Fietkau R, Hohenberger W, Hess C, Becker H, Sauer R, Wittekind C, Rödel C (2015): Downstage migration after neoadjuvant chemoradiotherapy for rectal cancer: the reverse of the Will Rogers phenomenon? Cancer $\underline{121}$, 1724-1727

Fokas E, Ströbel P, Fietkau R, Ghadimi M, Liersch T, Grabenbauer GG, Hartmann A, Kaufmann M, Sauer R, Graeven $U$ et al. (2017): Tumor regression grading after preoperative chemoradiotherapy as a prognostic factor and individual-level surrogate for disease-free survival in rectal cancer. J Natl Cancer Inst 109

Fokas E, Fietkau R, Hartmann A, Hohenberger W, Grützmann R, Ghadimi M, Liersch T, Ströbel P, Grabenbauer GG, Graeven U et al. (2018): Neoadjuvant rectal score as individual-level surrogate for disease-free survival in rectal cancer in the CAO/ARO/AIO-04 randomized phase III trial. Ann Oncol 29, 1521-1527 
Fokas E, Allgäuer M, Polat B, Klautke G, Grabenbauer GG, Fietkau R, Kuhnt T, Staib L, Brunner T, Grosu AL et al. (2019): Randomized phase II trial of chemoradiotherapy plus induction or consolidation chemotherapy as total neoadjuvant therapy for locally advanced rectal cancer: CAO/ARO/AIO-12. J Clin Oncol 37, 3212-3222

Folprecht G, Köhne CH (2004): The role of new agents in the treatment of colorectal cancer. Oncology $\underline{66}, 1-$ 17

Folprecht G, Lutz MP, Schöffski P, Seufferlein T, Nolting A, Pollert P, Köhne CH (2006): Cetuximab and irinotecan/5-fluorouracil/folinic acid is a safe combination for the first-line treatment of patients with epidermal growth factor receptor expressing metastatic colorectal carcinoma. Ann Oncol 17, 450-456

Frickhofen N, Beck FJ, Jung B, Fuhr HG, Andrasch H, Sigmund M (2002): Capecitabine can induce acute coronary syndrome similar to 5-fluorouracil. Ann Oncol 13, 797-801

Gains A, Whiting J: Robert Weinberg and the search for the cause of cancer. Mitchell Lane, Bear, Del. 2002

Gamelin E, Boisdron-Celle M, Morel A (2007): Dépistage des patients déficitaires en dihydropyrimidine déhydrogénase avant traitement par fluoropyrimidines. Therapie $\underline{62}, 99-103$

Gamelin L, Boisdron-Celle M, Delva R, Guérin-Meyer V, Ifrah N, Morel A, Gamelin E (2004): Prevention of oxaliplatin-related neurotoxicity by calcium and magnesium infusions: a retrospective study of 161 patients receiving oxaliplatin combined with 5-fluorouracil and leucovorin for advanced colorectal cancer. Clin Cancer Res $\underline{10}$, 4055-4061

García-Aguilar J, Hernandez de Anda E, Sirivongs P, Lee SH, Madoff RD, Rothenberger DA (2003): A pathologic complete response to preoperative chemoradiation is associated with lower local recurrence and improved survival in rectal cancer patients treated by mesorectal excision. Dis Colon Rectum 46, 298-304

Garcia-Aguilar J, Chow OS, Smith DD, Marcet JE, Cataldo PA, Varma MG, Kumar AS, Oommen S, Coutsoftides T, Hunt SR et al. (2015): Effect of adding mFOLFOX6 after neoadjuvant chemoradiation in locally advanced rectal cancer: a multicentre, phase 2 trial. Lancet Oncol 16, 957-966

Gehoff A, Basten O, Sprenger T, Conradi LC, Bismarck C, Bandorski D, Merkelbach-Bruse S, SchneiderStock R, Stoehr R, Wirtz RM et al. (2012): Optimal lymph node harvest in rectal cancer (UICC stages II and III) after preoperative 5-FU-based radiochemotherapy. Acetone compression is a new and highly efficient method. Am J Surg Pathol 36, 202-213

Gerard JP, Glynne-Jones R, Blackstock W, Aschele C, van de Velde C (2003): Radiochemotherapy in rectal

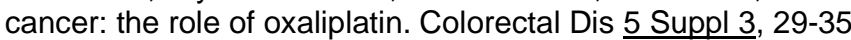

Gervaz P, Bouzourene H, Cerottini JP, Chaubert P, Benhattar J, Secic M, Wexner S, Givel JC, Belin B (2001): Dukes $\mathrm{B}$ colorectal cancer: distinct genetic categories and clinical outcome based on proximal or distal tumor location. Dis Colon Rectum 44, 364-373

Glynne-Jones R, Mawdsley S, Novell JR (2006): The clinical significance of the circumferential resection margin following preoperative pelvic chemo-radiotherapy in rectal cancer: why we need a common language. Colorectal Dis $\underline{8}, 800-807$

Glynne-Jones R, Counsell N, Quirke P, Mortensen N, Maraveyas A, Meadows HM, Ledermann J, SebagMontefiore D (2014): Chronicle: results of a randomised phase III trial in locally advanced rectal cancer after neoadjuvant chemoradiation randomising postoperative adjuvant capecitabine plus oxaliplatin (XELOX) versus control. Ann Oncol 25, 1356-1362

Goldberg RM, Sargent DJ, Morton RF, Fuchs CS, Ramanathan RK, Williamson SK, Findlay BP, Pitot HC, Alberts SR (2004): A randomized controlled trial of fluorouracil plus leucovorin, irinotecan, and oxaliplatin combinations in patients with previously untreated metastatic colorectal cancer. J Clin Oncol 22, 23-30

Goldstein NS, Soman A, Sacksner J (1999): Disparate surgical margin lengths of colorectal resection specimens between in vivo and in vitro measurements. The effects of surgical resection and formalin fixation on organ shrinkage. Am J Clin Pathol 111, 349-351

Gosens MJEM, Klaassen RA, Tan-Go I, Rutten HJT, Martijn H, van den Brule AJC, Nieuwenhuijzen GAP, van Krieken JHJM, Nagtegaal ID (2007): Circumferential margin involvement is the crucial prognostic factor after multimodality treatment in patients with locally advanced rectal carcinoma. Clin Cancer Res $\underline{13}$, 6617-6623

Grade M, Beham AW, Schüler P, Kneist W, Ghadimi BM (2016): Pelvic intraoperative neuromonitoring during robotic-assisted low anterior resection for rectal cancer. J Robot Surg 10, 157-160

Graeven U, Andre N, Schmiegel W (2004): Aktuelle Therapiemöglichkeiten des kolorektalen Karzinoms. Z Gastroenterol 느, 1399-1407 
Graham MA, Lockwood GF, Greenslade D, Brienza S, Bayssas M, Gamelin E (2000): Clinical pharmacokinetics of oxaliplatin: a critical review. Clin Cancer Res $\underline{6}, 1205-1218$

Gramont A de, Bosset JF, Milan C, Rougier P, Bouché O, Etienne PL, Morvan F, Louvet C, Guillot T, François E et al. (1997): Randomized trial comparing monthly low-dose leucovorin and fluorouracil bolus with bimonthly high-dose leucovorin and fluorouracil bolus plus continuous infusion for advanced colorectal cancer: a French intergroup study. J Clin Oncol $\underline{15}, 808-815$

Gramont A de, Figer A, Seymour M, Homerin M, Hmissi A, Cassidy J, Boni C, Cortes-Funes H, Cervantes A, Freyer $\mathrm{G}$ et al. (2000): Leucovorin and fluorouracil with or without oxaliplatin as first-line treatment in advanced colorectal cancer. J Clin Oncol $\underline{18}, 2938-2947$

Greene FL, Sobin LH (2002): The TNM system: our language for cancer care. J Surg Oncol $\underline{80}, 119-120$

Gresham G, Cheung WY, Speers C, Woods R, Kennecke H (2015): Time to adjuvant chemotherapy and survival outcomes among patients with stage 2 to 3 rectal cancer treated with preoperative chemoradiation. Clin Colorectal Cancer 14, 41-45

Grothey A, Sobrero AF, Shields AF, Yoshino T, Paul J, Taieb J, Souglakos J, Shi Q, Kerr R, Labianca R et al. (2018): Duration of adjuvant chemotherapy for stage III colon cancer. N Engl J Med $\underline{378}, 1177-1188$

Haller DG, Tabernero J, Maroun J, de Braud F, Price T, van Cutsem E, Hill M, Gilberg F, Rittweger K, Schmoll HJ (2011): Capecitabine plus oxaliplatin compared with fluorouracil and folinic acid as adjuvant therapy for stage III colon cancer. J Clin Oncol 29, 1465-1471

Han R, Yang YM, Dietrich J, Luebke A, Mayer-Pröschel M, Noble M (2008): Systemic 5-fluorouracil treatment causes a syndrome of delayed myelin destruction in the central nervous system. J Biol $\underline{7}, 12$

Harewood GC, Wiersema MJ, Nelson H, Maccarty RL, Olson JE, Clain JE, Ahlquist DA, Jondal ML (2002): A prospective, blinded assessment of the impact of preoperative staging on the management of rectal cancer. Gastroenterology $\underline{123}, 24-32$

Heald RJ (1998): Total mesorectal excision. The new european gold standard. G Chir $\underline{19}$, 253-255

Heald RJ, Husband EM, Ryall RD (1982): The mesorectum in rectal cancer surgery - the clue to pelvic recurrence? Br J Surg $\underline{69}, 613-616$

Heald RJ, Smedh RK, Kald A, Sexton R, Moran BJ (1997): Abdominoperineal excision of the rectum - an endangered operation. Norman Nigro Lectureship. Dis Colon Rectum $\underline{40}$, 747-751

Heald RJ, Moran BJ, Ryall RD, Sexton R, MacFarlane JK (1998): Rectal cancer: the Basingstoke experience of total mesorectal excision, 1978-1997. Arch Surg $\underline{133}$, 894-899

Heidelberger C (1975): Fluorinated Pyrimidines and Their Nucleosides; In: Antineoplastic and Immunosuppressive Agents; hrsg. v. Sartorelli AC, Johns DG: Springer Berlin Heidelberg. Berlin, Heidelberg 1975, $193-$ 231

Heidelberger C, Chaudhuri NK, Danneberg P, Mooren D, Griesbach L, Duschinsky R, Schnitzer RJ, Pleven E, Scheiner J (1957): Fluorinated pyrimidines, a new class of tumour-inhibitory compounds. Nature 179, 663-666

Hermanek P (1999a): Impact of surgeon's technique on outcome after treatment of rectal carcinoma. Dis CoIon Rectum $\underline{42}$, 559-562

Hermanek P (1999b): Entwicklung von Standards und Leitlinien durch die Deutsche Krebsgesellschaft. Z Arztl Fortbild Qualitatssich $\underline{93}$, 9-13

Hermanek P, Hohenberger W, Klimpfinger M, Köckerling F, Papadopoulos T (2003): The pathological assessment of mesorectal excision: implications for further treatment and quality management. Int $\mathrm{J}$ Colorectal Dis $\underline{18}, 335-341$

Hermanek P, Merkel S, Ptok H, Hohenberger W (2015): Qualität der Mesorektumexzision ("plane of surgery") - Welche Qualitätsziele sind angemessen? Zentralbl Chir 140, 627-632

Hida J, Yasutomi M, Maruyama T, Fujimoto K, Uchida T, Okuno K (1997): Lymph node metastases detected in the mesorectum distal to carcinoma of the rectum by the clearing method: justification of total mesorectal excision. J Am Coll Surg 184, 584-588

Hofheinz R-D, Wenz F, Post S, Matzdorff A, Laechelt S, Hartmann JT, Müller L, Link H, Moehler M, Kettner E et al. (2012): Chemoradiotherapy with capecitabine versus fluorouracil for locally advanced rectal cancer: a randomised, multicentre, non-inferiority, phase 3 trial. Lancet Oncol $\underline{13}, 579-588$ 
Hofheinz RD, Arnold, Dirk, Borner, Markus, Folprecht G, Graeven U, Hebart H, Hegewisch-Becker S, Heinemann V, Meybier T, Pritzkuleit R et al. (2018a): Kolonkarzinom.

https://www.onkopedia.com/de/onkopedia/guidelines/kolonkarzinom/@@guideline/html/index.html\#litlD0EO5 Al; abgerufen am: 13.01.2020

Hofheinz RD, Arnold, Dirk, Borner, Markus, Folprecht G, Graeven U, Hebart H, Hegewisch-Becker S, Heinemann V, Meybier T, Pritzkuleit R et al. (2018b): Rektumkarzinom.

https://www.onkopedia.com/de/onkopedia/guidelines/rektumkarzinom/@@guideline/html/index.html; abgerufen am: 13.01.2020

Hofheinz RD, Arnold D, Fokas E, Kaufmann M, Hothorn T, Folprecht G, Fietkau R, Hohenberger W, Ghadimi $M$, Liersch T et al. (2018c): Impact of age on the efficacy of oxaliplatin in the preoperative chemoradiotherapy and adjuvant chemotherapy of rectal cancer: a post hoc analysis of the CAO/ARO/AIO-04 phase III trial. Ann Oncol 29, 1793-1799

Hohenberger W, Lahmer G, Fietkau R, Croner RS, Merkel S, Göhl J, Sauer R (2009): Neoadjuvante Radiochemotherapie des Rektumkarzinoms. Chirurg 80, 294-302

Holch JW, Ricard I, Stintzing S, Modest DP, Heinemann V (2017): The relevance of primary tumour location in patients with metastatic colorectal cancer: A meta-analysis of first-line clinical trials. Eur J Cancer $\underline{70}, 87-98$

Holm T, Ljung A, Häggmark T, Jurell G, Lagergren J (2007): Extended abdominoperineal resection with gluteus maximus flap reconstruction of the pelvic floor for rectal cancer. $\mathrm{Br} \mathrm{J}$ Surg $\underline{94}, 232-238$

Hong YS, Nam BH, Kim KP, Kim JE, Park SJ, Park YS, Park JO, Kim SY, Kim TY, Kim JH et al. (2014): Oxaliplatin, fluorouracil, and leucovorin versus fluorouracil and leucovorin as adjuvant chemotherapy for locally advanced rectal cancer after preoperative chemoradiotherapy (ADORE): an open-label, multicentre, phase 2, randomised controlled trial. Lancet Oncol 15, 1245-1253

Hong YS, Kim SY, Lee JS, Nam BH, Kim KP, Kim JE, Park YS, Park JO, Baek JY, Kim TY et al. (2019): Oxaliplatin-based adjuvant chemotherapy for rectal cancer after preoperative chemoradiotherapy (ADORE): Long-term results of a randomized controlled trial. J Clin Oncol 37, 3111-3123

lacopetta B (2002): Are there two sides to colorectal cancer? Int J Cancer 101, 403-408

IARC, International Agency for Research on Cancer (IARC) (2018a): Germany: Source: Globocan 2018. https://gco.iarc.fr/today/data/factsheets/populations/276-germany-fact-sheets.pdf; abgerufen am: 31.01.2020

IARC, International Agency for Research on Cancer (IARC) (2018b): World: Source: Globocan 2018. https://gco.iarc.fr/today/data/factsheets/populations/900-world-fact-sheets.pdf; abgerufen am: 31.01.2020

Jass JR, Sobin LH (1989): Histological Typing of Intestinal Tumours (World Health Organization), Second edition; Springer-Verlag, Berlin Heidelberg 1989

Kaatsch P, Spix C, Katalinic A, Hentschel S, Luttmann S, Stegmaier C, Caspritz S, Christ M, Ernst A, Folkerts $\mathrm{J}$ et al (2015): Beiträge zur Gesundheitsberichterstattung des Bundes - Krebs in Deutschland 2011/2012 (Gesundheitsberichterstattung für Deutschland), 10. Ausgabe; Robert Koch-Institut, Berlin 2015

Kaldate RR, Haregewoin A, Grier CE, Hamilton SA, McLeod HL (2012): Modeling the 5-fluorouracil area under the curve versus dose relationship to develop a pharmacokinetic dosing algorithm for colorectal cancer patients receiving FOLFOX6. Oncologist 17, 296-302

Karagkounis G, Thai L, Mace AG, Wiland H, Pai RK, Steele SR, Church JM, Kalady MF (2019): Prognostic Implications of Pathological Response to Neoadjuvant Chemoradiation in Pathologic Stage III Rectal Cancer. Ann Surg 269, 1117-1123

Khrizman P, Niland JC, ter Veer A, Milne D, Bullard Dunn K, Carson WE, Engstrom PF, Shibata S, Skibber JM, Weiser MR et al. (2013): Postoperative adjuvant chemotherapy use in patients with stage II/III rectal cancer treated with neoadjuvant therapy: a national comprehensive cancer network analysis. J Clin Oncol $\underline{31}, 30$ 38

Kidani Y, Inagaki K, ligo M, Hoshi A, Kuretani K (1978a): Antitumor activity of 1,2-diaminocyclohexaneplatinum complexes against sarcoma-180 ascites form. J Med Chem 21, 1315-1318

Kidani Y, Okamoto K, Noji M, Tashiro T (1978b): Antitumor activity of platinum (II) complexes of 1-amino-2aminomethylcyclohexane isomers. Gan $\underline{69}, 863-864$

Kim DW, Kim DY, Kim TH, Jung KH, Chang HJ, Sohn DK, Lim SB, Choi HS, Jeong SY, Park JG (2006): Is T classification still correlated with lymph node status after preoperative chemoradiotherapy for rectal cancer? Cancer $\underline{106}$, 1694-1700 
Kim SH, Chang HJ, Kim DY, Park JW, Baek JY, Kim SY, Park SC, Oh JH, Yu A, Nam BH (2016): What is the ideal tumor regression grading system in rectal cancer patients after preoperative chemoradiotherapy? Cancer Res Treat $\underline{48}, 998-1009$

Kim YW, Cha SW, Pyo J, Kim NK, Min BS, Kim MJ, Kim H (2009): Factors related to preoperative assessment of the circumferential resection margin and the extent of mesorectal invasion by magnetic resonance imaging in rectal cancer: a prospective comparison study. World J Surg $\underline{33}, 1952-1960$

Kitz J, Fokas E, Beissbarth T, Ströbel P, Wittekind C, Hartmann A, Rüschoff J, Papadopoulos T, Rösler E, Ortloff-Kittredge P et al. (2018): Association of plane of total mesorectal excision with prognosis of rectal cancer: Secondary analysis of the CAO/ARO/AIO-04 Phase 3 randomized clinical trial. JAMA Surg 153, e181607

Koenig H, Patel A (1970a): Biochemical basis for fluorouracil neurotoxicity. The role of krebs cycle inhibition by fluoroacetate. Arch Neurol $\underline{23}, 155-160$

Koenig H, Patel A (1970b): The acute cerebellar syndrome in 5-fluorouracil chemotherapy: a manifestation of fluoroacetate intoxication. Neurology 20, 416

Köhne $\mathrm{CH}$, van Cutsem E, Wils J, Bokemeyer C, El-Serafi M, Lutz MP, Lorenz M, Reichardt P, Rückle-Lanz $\mathrm{H}$, Frickhofen $\mathrm{N}$ et al. (2005): Phase III study of weekly high-dose infusional fluorouracil plus folinic acid with or without irinotecan in patients with metastatic colorectal cancer: European Organisation for Research and Treatment of Cancer Gastrointestinal Group Study 40986. J Clin Oncol 23, 4856-4865

Kopetz S, Chang GJ, Overman MJ, Eng C, Sargent DJ, Larson DW, Grothey A, Vauthey JN, Nagorney DM, McWilliams RR (2009): Improved survival in metastatic colorectal cancer is associated with adoption of hepatic resection and improved chemotherapy. J Clin Oncol 27, 3677-3683

Krebsfrüherkennungsrichtlinie: siehe Richtlinien des Bundesausschusses der Ärzte und Krankenkassen über die Früherkennung von Krebserkrankungen 2002

Krook JE, Moertel CG, Gunderson LL, Wieand HS, Collins RT, Beart RW, Kubista TP, Poon MA, Meyers WC, Mailliard JA (1991): Effective surgical adjuvant therapy for high-risk rectal carcinoma. N Engl J Med $\underline{324}$, 709715

Kwok H, Bissett IP, Hill GL (2000): Preoperative staging of rectal cancer. Int J Colorectal Dis $\underline{15}$, 9-20

Labianca R, Beretta G, Clerici M, Fraschini P, Luporini G (1982): Cardiac toxicity of 5-fluorouracil: a study on 1083 patients. Tumori $\underline{68}, 505-510$

Le DT, Uram JN, Wang H, Bartlett BR, Kemberling H, Eyring AD, Skora AD, Luber BS, Azad NS, Laheru D et al. (2015): PD-1 blockade in tumors with mismatch-repair deficiency. N Engl J Med 372, 2509-2520

Le Voyer TE, Sigurdson ER, Hanlon AL, Mayer RJ, Macdonald JS, Catalano PJ, Haller DG (2003): Colon cancer survival is associated with increasing number of lymph nodes analyzed: a secondary survey of intergroup trial INT-0089. J Clin Oncol 21, 2912-2919

Leitlinienprogramm Onkologie (Deutsche Krebsgesellschaft, Deutsche Krebshilfe, AWMF) (2019): S3-Leitlinie Kolorektales Karzinom, Langversion 2.1, Januar 2019, AWMF Registrierungsnummer: 021/007OL. http://www.leitlinienprogramm-onkologie.de/leitlinien/kolorektales-karzinom/; abgerufen am: 16.01.2020

Li FY, Lai MD (2009): Colorectal cancer, one entity or three. J Zhejiang Univ Sci B 10, 219-229

Liang HQ, Dong ZY, Liu ZJ, Luo J, Zeng Q, Liao PY, Wu DH (2019): Efficacy and safety of consolidation chemotherapy during the resting period in patients with local advanced rectal cancer. Oncol Lett $\underline{17}, 1655-$ 1663

Liao ZY, Sordet O, Zhang HL, Kohlhagen G, Antony S, Gmeiner WH, Pommier Y (2005): A novel polypyrimidine antitumor agent FdUMP10 induces thymineless death with topoisomerase I-DNA complexes. Cancer Res $\underline{65}, 4844-4851$

Liersch T, Langer C, Jakob C, Müller D, Ghadimi BM, Siemer A, Markus PM, Füzesi L, Becker H (2003): Präoperative Diagnostik beim lokal fortgeschrittenen Rektumkarzinom (or $=\mathrm{T} 3$ oder $\mathrm{N}+$ ). Was leistet die Endosonographie gegenüber der Computertomographie im Staging und Restaging (nach neoadjuvanter Radio/Chemotherapie)? Chirurg $\underline{74}, 224-234$

Liersch T, Langer C, Ghadimi BM, Becker H (2005): Aktuelle Behandlungsstrategien beim Rektumkarzinom. Chirurg $\underline{76}$, 309-32; quiz 333-4

Liersch T, Langer C, Ghadimi B, Rothe H, Hess C, Becker H (2006a): Technik der mesorektaler Exzision beim Rektumkarzinom - immer noch ein aktuelles Thema? Viszeralchirurgie 41, 313-323 
Liersch T, Rothe H, Ghadimi B, Langer C, Becker H (2006b): Chirurgische und pathologische Qualitätssicherung beim Rektumkarzinom - eine besondere Herausforderung mit Einfluss auf die Therapie? Viszeralchirurgie $\underline{41}, 324-332$

Liersch T, Rothe H, Rödel C, Ghadimi B, Becker H, Langer C (2007a): Chirurgie des Rektumkarzinoms Qualitätssicherung. Viszeralchirurgie $\underline{42}, 66-72$

Liersch T, Becker H, Langer C (2007b): Rektumkarzinom. AVCup 1, 41-69

Liersch T, Rothe H, Ghadimi BM, Becker H (2009): Therapie beim lokal fortgeschrittenen Rektumkarzinom. Ansätze zur Individualisierung. Chirurg $\underline{80}, 281-293$

Locker GY, Hamilton S, Harris J, Jessup JM, Kemeny N, Macdonald JS, Somerfield MR, Hayes DF, Bast RC (2006): ASCO 2006 update of recommendations for the use of tumor markers in gastrointestinal cancer. J Clin Oncol 24, 5313-5327

Lokich JJ, Ahlgren JD, Gullo JJ, Philips JA, Fryer JG (1989): A prospective randomized comparison of continuous infusion fluorouracil with a conventional bolus schedule in metastatic colorectal carcinoma: a Mid-Atlantic Oncology Program Study. J Clin Oncol ㄱ, 425-432

Longley DB, Harkin DP, Johnston PG (2003): 5-fluorouracil: mechanisms of action and clinical strategies. Nat Rev Cancer $\underline{3}$, 330-338

Lönn U, Lönn S (1984): Interaction between 5-fluorouracil and DNA of human colon adenocarcinoma. Cancer Res $\underline{44}, 3414-3418$

Luengo-Fernandez R, Leal J, Gray A, Sullivan R (2013): Economic burden of cancer across the european union: a population-based cost analysis. The Lancet Oncology 14, 1165-1174

Luo FR, Wyrick SD, Chaney SG (1999): Biotransformations of oxaliplatin in rat blood in vitro. J. Biochem. Mol. Toxicol. 13, 159-169

Lynch HT, Lynch PM, Lanspa SJ, Snyder CL, Lynch JF, Boland CR (2009): Review of the Lynch syndrome: history, molecular genetics, screening, differential diagnosis, and medicolegal ramifications. Clin Genet $\underline{76}, 1-$ 18

Maas M, Nelemans PJ, Valentini V, Das P, Rödel C, Kuo LJ, Calvo FA, García-Aguilar J, Glynne-Jones R, Haustermans $\mathrm{K}$ et al. (2010): Long-term outcome in patients with a pathological complete response after chemoradiation for rectal cancer: a pooled analysis of individual patient data. The Lancet Oncology 11, 835844

Maas M, Nelemans PJ, Valentini V, Crane CH, Capirci C, Rödel C, Nash GM, Kuo LJ, Glynne-Jones R, García-Aguilar $\mathrm{J}$ et al. (2015): Adjuvant chemotherapy in rectal cancer: defining subgroups who may benefit after neoadjuvant chemoradiation and resection: a pooled analysis of 3,313 patients. Int J Cancer $137,212-220$

Mace AG, Pai RK, Stocchi L, Kalady MF (2015): American Joint Committee on Cancer and College of American Pathologists regression grade: a new prognostic factor in rectal cancer. Dis Colon Rectum $\underline{58}, 32-44$

Mackay SG, Pager CK, Joseph D, Stewart PJ, Solomon MJ (2003): Assessment of the accuracy of transrectal ultrasonography in anorectal neoplasia. Br J Surg $\underline{90}, 346-350$

Mandel HG (1981): The target cell determinants of the antitumor actions of 5-FU: does FU incorporation into RNA play a role? Cancer Treat Rep 65 Suppl 3, 63-71

Mani S, Graham MA, Bregman DB, Ivy P, Chaney SG (2002): Oxaliplatin: a review of evolving concepts. Cancer Invest 20, 246-263

Marco MR, Zhou L, Patil S, Marcet JE, Varma MG, Oommen S, Cataldo PA, Hunt SR, Kumar A, Herzig DO et al. (2018): Consolidation mFOLFOX6 chemotherapy after chemoradiotherapy improves survival in patients with locally advanced rectal cancer: Final results of a multicenter phase II trial. Dis Colon Rectum $61,1146-$ 1155

Mari GM, Maggioni D, Crippa J, Costanzi ATM, Scotti MA, Giardini V, Garancini M, Cocozza E, Borroni G, Benzoni I et al. (2019): Compliance to adjuvant chemotherapy of patients who underwent surgery for rectal cancer: Report from a multi-institutional research network. World J Surg $\underline{43}, 2544-2551$

Martijnse IS, Dudink RL, Kusters M, Vermeer TA, West NP, Nieuwenhuijzen GA, van Lijnschoten I, Martijn H, Creemers GJ, Lemmens VE et al. (2012): T3 and T4 rectal cancer patients seem to benefit from the addition of oxaliplatin to the neoadjuvant chemoradiation regimen. Ann Surg Oncol $\underline{19}$, 392-401 
Martling A, Cedermark B, Johansson H, Rutqvist LE, Holm T (2002): The surgeon as a prognostic factor after the introduction of total mesorectal excision in the treatment of rectal cancer. Br J Surg $\underline{89}, 1008-1013$

Mason AY (1976a): President's address. Rectal cancer: the spectrum of selective surgery. Proc R Soc Med $\underline{69}, 237-244$

Mason AY (1976b): Selective surgery for carcinoma of the rectum. Aust N Z J Surg $\underline{46}, 322-329$

Maurer U, Härle M, Jungius KP (1996): 5-fluorouracil: Auslöser eines Herzinfarkts als Todesursache bei kombinierter Radiochemotherapie? Strahlenther Onkol 172, 257-259

Mawdsley S, Glynne-Jones R, Grainger J, Richman P, Makris A, Harrison M, Ashford R, Harrison RA, Osborne M, Livingstone $\mathrm{Jl}$ et al. (2005): Can histopathologic assessment of circumferential margin after preoperative pelvic chemoradiotherapy for T3-T4 rectal cancer predict for 3-year disease-free survival? Int J Radiat Oncol Biol Phys $\underline{63}, 745-752$

Mayer RJ, van Cutsem E, Falcone A, Yoshino T, Garcia-Carbonero R, Mizunuma N, Yamazaki K, Shimada Y, Tabernero J, Komatsu Y et al. (2015): Randomized trial of TAS-102 for refractory metastatic colorectal cancer. N Engl J Med 372, 1909-1919

MERCURY Study Group (2007): Extramural depth of tumor invasion at thin-section MR in patients with rectal cancer: results of the MERCURY study. Radiology $\underline{243}, 132-139$

Merkel S, Mansmann U, Siassi M, Papadopoulos T, Hohenberger W, Hermanek P (2001): The prognostic inhomogeneity in pT3 rectal carcinomas. Int J Colorectal Dis $\underline{16}, 298-304$

Merkel S, Weber K, Croner RS, Golcher H, Göhl J, Agaimy A, Semrau S, Siebler J, Wein A, Hohenberger W et al. (2016): Distant metastases in colorectal carcinoma: A proposal for a new M1 subclassification. Eur J Surg Oncol $\underline{42}, 1337-1342$

Meyer CC, Calis KA, Burke LB, Walawander CA, Grasela TH (1997): Symptomatic cardiotoxicity associated with 5-fluorouracil. Pharmacotherapy $\underline{17}, 729-736$

Milano G, Etienne MC, Cassuto-Viguier E, Renée N, Bousselet M, Guillot T, Lecompte D (1992): Long-term stability of 5-fluorouracil and folinic acid admixtures. Eur J Cancer 29A, 129-132

Miyakura Y, Sugano K, Konishi F, Ichikawa A, Maekawa M, Shitoh K, Igarashi S, Kotake K, Koyama Y, Nagai $\mathrm{H}$ (2001): Extensive methylation of hMLH1 promoter region predominates in proximal colon cancer with microsatellite instability. Gastroenterology 121, 1300-1309

Moertel CG, Fleming TR, Macdonald JS, Haller DG, Laurie JA, Goodman PJ, Ungerleider JS, Emerson WA, Tormey DC, Glick JH (1990): Levamisole and fluorouracil for adjuvant therapy of resected colon carcinoma. N Engl J Med $\underline{322}, 352-358$

Monga DK, O'Connell MJ (2006): Surgical adjuvant therapy for colorectal cancer: current approaches and future directions. Ann Surg Oncol 13, 1021-1034

Munding J, Tannapfel A (2009): Pathogenese des sporadischen und hereditären Kolonkarzinoms. Onkologe $15,1182-1192$

Mutschler E: Arzneimittelwirkungen: Lehrbuch der Pharmakologie und Toxikologie; mit einführenden Kapiteln in die Anatomie, Physiologie und Pathophysiologie, 8. völlig neu bearb. und erw. Aufl; Wiss. Verl. -Ges, Stuttgart 2001

Mutschler E, Geisslinger, G, Kroemer, HK, Ruth, P, Schäfer-Korting, M: Arzneimittelwirkungen: Lehrbuch der Pharmakologie und Toxikologie, 9. vollständig neu bearbeitete und erweiterte Auflage; Wissenschaftliche Verlagsgesellschaft, Stuttgart 2008

Nagtegaal ID, Marijnen CAM, Kranenbarg EK, van de Velde CJH, van Krieken JHJM (2002a): Circumferential margin involvement is still an important predictor of local recurrence in rectal carcinoma: not one millimeter but two millimeters is the limit. Am J Surg Pathol 26, 350-357

Nagtegaal ID, van de Velde CJH, van der Worp E, Kapiteijn E, Quirke P, van Krieken JHJM (2002b): Macroscopic evaluation of rectal cancer resection specimen: clinical significance of the pathologist in quality control. J Clin Oncol 20, 1729-1734

Nagtegaal ID, van de Velde CJH, Marijnen CAM, van Krieken JHJM, Quirke P (2005): Low rectal cancer: a call for a change of approach in abdominoperineal resection. J Clin Oncol $\underline{23}, 9257-9264$ 
Nagtegaal ID, Knijn N, Hugen N, Marshall HC, Sugihara K, Tot T, Ueno H, Quirke P (2017): Tumor deposits in colorectal cancer: improving the value of modern staging-A systematic review and meta-analysis. J Clin Oncol $\underline{35}, 1119-1127$

$\mathrm{NCl}$, National Cancer Institute: (2010) Common Terminology Criteria for Adverse Events (CTCAE) https://ctep.cancer.gov/protocolDevelopment/electronic_applications/ctc.htm; abgerufen am: 27.10.2019

Neri E, Giusti P, Battolla L, Vagli P, Boraschi P, Lencioni R, Caramella D, Bartolozzi C (2002): Colorectal cancer: role of CT colonography in preoperative evaluation after incomplete colonoscopy. Radiology $\underline{223}$, 615619

Niekel MC, Bipat S, Stoker J (2010): Diagnostic imaging of colorectal liver metastases with CT, MR imaging, FDG PET, and/or FDG PET/CT: a meta-analysis of prospective studies including patients who have not previously undergone treatment. Radiology $\underline{257}, 674-684$

NGTATG, Nordic Gastrointestinal Tumor Adjuvant Therapy Group (NGTATG) (1992): Expectancy or primary chemotherapy in patients with advanced asymptomatic colorectal cancer: a randomized trial. J Clin Oncol $\underline{10}$, 904-911

Online Mendelian Inheritance in Man $®$ (OMIM): \# 120435 Lynch Syndrome I.

https://www.omim.org/entry/120435?search=\%23\%20120435\&highlight=120435; abgerufen am: 13.01 .2020

Online Mendelian Inheritance in Man ${ }^{\circledR}$ (OMIM): \# 175100 Familal Adenomatous Polyposis1, FAP1. https://www.omim.org/entry/175100; abgerufen am: 13.01.2020

Patel B, Kloner RA, Ensley J, Al-Sarraf M, Kish J, Wynne J (1987): 5-Fluorouracil cardiotoxicity: left ventricular dysfunction and effect of coronary vasodilators. Am J Med Sci 294, 238-243

Peeters KCMJ, Marijnen CAM, Nagtegaal ID, Kranenbarg EK, Putter H, Wiggers T, Rutten H, Pahlman L, Glimelius B, Leer JW et al. (2007): The TME trial after a median follow-up of 6 years: increased local control but no survival benefit in irradiated patients with resectable rectal carcinoma. Ann Surg 246, 693-701

Perry WB, Connaughton JC (2007): Abdominoperineal resection: how is it done and what are the results? Clin Colon Rectal Surg 20, 213-220

Petersen SH, Harling H, Kirkeby LT, Wille-Jørgensen P, Mocellin S (2012): Postoperative adjuvant chemotherapy in rectal cancer operated for cure. Cochrane Database Syst Rev, CD004078

Pinedo HM, Peters GF (1988): Fluorouracil: biochemistry and pharmacology. J Clin Oncol $\underline{6}, 1653-1664$

Pizzorno G, Handschumacher RE, Cheng YC: Pyrimidine and purine antimetabolites. In: Holland JF, Frei E, Bast RC, Kufe DW, Morton DL and Weichselbaum RR (Hrsg): Cancer Medicine. 4. Auflage; Lea \& Febiger, Philadelphia 1997, 924-948

Polanco PM, Mokdad AA, Zhu H, Choti MA, Huerta S (2018): Association of adjuvant chemotherapy with overall survival in patients with rectal cancer and pathologic complete response following neoadjuvant chemotherapy and resection. JAMA Oncol $\underline{4}, 938-943$

Polk A, Vaage-Nilsen M, Vistisen K, Nielsen DL (2013): Cardiotoxicity in cancer patients treated with 5fluorouracil or capecitabine: a systematic review of incidence, manifestations and predisposing factors. Cancer Treat Rev $\underline{39}$, 974-984

Polk A, Vistisen K, Vaage-Nilsen M, Nielsen DL (2014): A systematic review of the pathophysiology of 5fluorouracil-induced cardiotoxicity. BMC Pharmacol Toxicol $\underline{15}, 47$

Prentice RL (1989): Surrogate endpoints in clinical trials: definition and operational criteria. Stat Med $\underline{8}$, 431440

PubChem, National Center for Biotechnology Information. PubChem Database: Leucovorin: compound summary. https://pubchem.ncbi.nlm.nih.gov/compound/Leucovorin; abgerufen am: 12.01.2020

Puli SR, Reddy JBK, Bechtold ML, Choudhary A, Antillon MR, Brugge WR (2009): Accuracy of endoscopic ultrasound to diagnose nodal invasion by rectal cancers: a meta-analysis and systematic review. Ann Surg Oncol 16, 1255-1265

Punt CJA, Buyse M, Köhne CH, Hohenberger P, Labianca R, Schmoll HJ, Påhlman L, Sobrero A, Douillard JY (2007): Endpoints in adjuvant treatment trials: a systematic review of the literature in colon cancer and proposed definitions for future trials. J Natl Cancer Inst $\underline{99}$, 998-1003 
Quack H: 5-Flourouracil-Spiegelbestimmung unter neoadjuvanter Radiochemotherapie und adjuvanter Chemotherapie beim lokal fortgeschrittenen Rektumkarzinom: Analyse der pharmakologischen Variabilität von 5Fluorouracil mittels Nanopartikelimmunoassays. Dissertation Georg-August-Universität Göttingen 2015

Quack H, Erpenbeck L, Wolff HA, Sprenger T, Seitz CS, Schön MP, Neumann S, Stanek K, Ghadimi BM, Michels B et al. (2013): Oxaliplatin-Induced leukocytoclastic vasculitis under adjuvant chemotherapy for colorectal cancer: two cases of a rare adverse event. Case Rep Oncol $\underline{6}, 609-615$

Quasar CG, Gray R, Barnwell J, McConkey C, Hills RK, Williams NS, Kerr DJ (2007): Adjuvant chemotherapy versus observation in patients with colorectal cancer: a randomised study. Lancet $\underline{370}, 2020-2029$

Quirke P (2003): Training and quality assurance for rectal cancer: 20 years of data is enough. Lancet Oncol $\underline{4}$, 695-702

Quirke P, Dixon MF, Durdey P, Williams NS (1986): Local recurrence of rectal adenocarcinoma due to inadequate surgical resection. The Lancet $\underline{328}, 996-999$

Quirke P, Steele R, Monson J, Grieve R, Khanna S, Couture J, O'Callaghan C, Myint AS, Bessell E, Thompson LC et al. (2009): Effect of the plane of surgery achieved on local recurrence in patients with operable rectal cancer: a prospective study using data from the MRC CR07 and NCIC-CTG CO16 randomised clinical trial. Lancet Oncol $\underline{373}, 821-828$

Raida M, Schwabe W, Häusler P, van Kuilenburg AB, van Gennip AH, Behnke D, Höffken K (2001): Prevalence of a common point mutation in the dihydropyrimidine dehydrogenase (DPD) gene within the 5-splice donor site of intron 14 in patients with severe 5 -fluorouracil (5-FU)- related toxicity compared with controls. Clin Cancer Res ㄱ, 2832-2839

Rau B, Brandl A, Pascher A, Raue W, Sugarbaker P (2017): Oligometastatic disease in the peritoneal space with gastrointestinal cancer. Visc Med $\underline{33}, 42-46$

Raymond E, Chaney SG, Taamma A, Cvitkovic E (1998): Oxaliplatin: a review of preclinical and clinical studies. Ann Oncol $\underline{9}, 1053-1071$

Raymond E, Faivre S, Chaney S, Woynarowski J, Cvitkovic E (2002): Cellular and molecular pharmacology of oxaliplatin. Mol Cancer Ther 1 , 227-235

Reich B (2006): Zielgerichtete Therapie mit Cetuximab: Metastaiertes kolorektales Karzinom. SatellitensymposiumsberichtGemeinsame Jahrestagung der Deutschen, Österreichischen und Schweizerischen Gesellschaften für Hämatologie und Onkologie, Hannover, 1. bis 5. Oktober 2005.

https://www.rosenfluh.ch/media/onkologie/2006/01/Metastasiertes-kolorektales-Karzinom.pdf; abgerufen am: 12.01.2020

Renzulli P, Laffer UT (2005): Learning curve: the surgeon as a prognostic factor in colorectal cancer surgery. Recent Results Cancer Res $\underline{165}$, 86-104

Renzulli P, Lowy A, Maibach R, Egeli RA, Metzger U, Laffer UT (2006): The influence of the surgeon's and the hospital's caseload on survival and local recurrence after colorectal cancer surgery. Surgery $\underline{139}$, 296-304

Richtlinien des Bundesausschusses der Ärzte und Krankenkassen über die Früherkennung von Krebserkrankungen ("Krebsfrüherkennungs-Richtlinien); In: Deutsches Ärzteblatt 2002, 2650-2653 https://www.aerzteblatt.de/archiv/34420/Bekanntmachungen-Richtlinien-des-

Bundes\%C2\%ADaus\%C2\%ADschusses-der-Aerzte-und-Krankenkassen-ueber-die-Frueherkennung-vonKrebserkrankungen-(-Krebsfrueherkennungs-Richtlinien-); abgerufen am 31.01.2020

RKI (2016): Bericht zum Krebsgeschehen in Deutschland 2016; RKI-Bib1 (Robert Koch-Institut) 28; https://edoc.rki.de/bitstream/handle/176904/3264/28oaKVmifOwDk.pdf?sequence=1\&isAllowed=y; abgerufen am 13.01.2020

Rödel C, Liersch T, Becker H, Fietkau R, Hohenberger W, Hothorn T, Graeven U, Arnold D, LangWelzenbach M, Raab HR et al. (2012): Preoperative chemoradiotherapy and postoperative chemotherapy with fluorouracil and oxaliplatin versus fluorouracil alone in locally advanced rectal cancer: initial results of the German CAO/ARO/AIO-04 randomised phase 3 trial. Lancet Oncol 13, 679-687

Rödel C, Graeven U, Fietkau R, Hohenberger W, Hothorn T, Arnold D, Hofheinz RD, Ghadimi M, Wolff HA, Lang-Welzenbach $\mathrm{M}$ et al. (2015): Oxaliplatin added to fluorouracil-based preoperative chemoradiotherapy and postoperative chemotherapy of locally advanced rectal cancer (the German CAO/ARO/AIO-04 study): final results of the multicentre, open-label, randomised, phase 3 trial. Lancet Oncol 16, 979-989 
Rödel F, Martin D, Balermpas P, Wieland U, Winkelmann R, Riekmann T, Falk S, Rödel C, Fokas E (2019): Modulation of radiation sensitivity and antitumor immunity by viral pathogenic factors: Implications for radioimmunotherapy. Biochim Biophys Acta Rev Cancer 1871, 126-137

Royston P, Parmar MKB (2013): Restricted mean survival time: an alternative to the hazard ratio for the design and analysis of randomized trials with a time-to-event outcome. BMC Med Res Methodol 13, 152

S3-Leitlinie Kolorektales Karzinom: siehe Leitlinienprogramm Onkologie 2019

Sahlmann CO, Homayounfar K, Niessner M, Dyczkowski J, Conradi LC, Braulke F, Meller B, Beißbarth T, Ghadimi BM, Meller J et al. (2017): Repeated adjuvant anti-CEA radioimmunotherapy after resection of colorectal liver metastases: safety, feasibility, and long-term efficacy results of a prospective phase 2 study. Cancer $123,638-649$

Saif MW, Choma A, Salamone SJ, Chu E (2009): Pharmacokinetically guided dose adjustment of 5 fluorouracil: a rational approach to improving therapeutic outcomes. J Natl Cancer Inst 101, 1543-1552

Sainato A, Cernusco Luna Nunzia V, Valentini V, Paoli A de, Maurizi ER, Lupattelli M, Aristei C, Vidali C, Conti M, Galardi A et al. (2014): No benefit of adjuvant fluorouracil leucovorin chemotherapy after neoadjuvant chemoradiotherapy in locally advanced cancer of the rectum (LARC): Long term results of a randomized trial (I-CNR-RT). Radiother Oncol 113, 223-229

Salzberg M, Herrmann R (2000): Das Hand-Fuss-Syndrom (palmar-plantare Erythrodysästhesie). Schweiz Med Wochenschr 130, 1413-1416

Sanderson ER (1980): Henri Hartmann and the Hartmann operation. Arch Surg $\underline{115}$, 792-793

Sandra-Petrescu F, Herrle F, Burkholder I, Kienle P, Hofheinz RD (2018): Influence of complete administration of adjuvant chemotherapy cycles on overall and disease-free survival in locally advanced rectal cancer: post hoc analysis of a randomized, multicenter, non-inferiority, phase 3 trial. BMC Cancer $\underline{18}, 369$

Sara JD, Kaur J, Khodadadi R, Rehman M, Lobo R, Chakrabarti S, Herrmann J, Lerman A, Grothey A (2018): 5-fluorouracil and cardiotoxicity: a review. Ther Adv Med Oncol 10, 1758835918780140

Saris CP, van de Vaart PJ, Rietbroek RC, Blommaert FA (1996): In vitro formation of DNA adducts by cisplatin, lobaplatin and oxaliplatin in calf thymus DNA in solution and in cultured human cells. Carcinogenesis $\underline{17}$, 2763-2769

Sauer R, Becker H, Hohenberger W, Rödel C, Wittekind C, Fietkau R, Martus P, Tschmelitsch J, Hager E, Hess CF et al. (2004): Preoperative versus postoperative chemoradiotherapy for rectal cancer. N Engl J Med $\underline{351}, 1731-1740$

Sauer R, Liersch T, Merkel S, Fietkau R, Hohenberger W, Hess C, Becker H, Raab HR, Villanueva MT, Witzigmann $\mathrm{H}$ et al. (2012): Preoperative versus postoperative chemoradiotherapy for locally advanced rectal cancer: results of the German CAO/ARO/AIO-94 randomized phase III trial after a median follow-up of 11 years. J Clin Oncol 30, 1926-1933

Scheeff ED, Briggs JM, Howell SB (1999): Molecular modeling of the intrastrand guanine-guanine DNA adducts produced by cisplatin and oxaliplatin. Mol Pharmacol $\underline{56}, 633-643$

Scheel AH, Reineke RA, Sprenger T, Lokka S, Kitz J, Ghadimi BM, Rüschoff J, Liersch T, Middel P (2015): Comprehensive lymph node morphometry in rectal cancer using acetone compression. J Clin Pathol $\underline{68}$, 458464

Scheffold N, Sucker C, Stockschläder M, Cyran J (2005): Kardiotoxizität von 5-Flurouracil. Onkologe 11, 223230

Schiffmann L, Eiken AK, Gock M, Klar E (2013): Is the lymph node ratio superior to the Union for International Cancer Control (UICC) TNM system in prognosis of colon cancer? World J Surg Oncol 11, 79

Schlitt A, Jordan K, Vordermark D, Schwamborn J, Langer T, Thomssen C (2014): Cardiotoxicity and oncological treatments. Dtsch Arztebl Int 111, 161-168

Schmiegel W, Reinacher-Schick A, Arnold D, Graeven U, Heinemann V, Porschen R, Riemann J, Rödel C, Sauer R, Wieser M et al. (2008): S3-Leitlinie "Kolorektales Karzinom" - Aktualisierung 2008. Z Gastroenterol $\underline{46}, 799-840$

Schmoll HJ, van Cutsem E, Stein A, Valentini V, Glimelius B, Haustermans K, Nordlinger B, van de Velde CJ, Balmana J, Regula J et al. (2012): ESMO Consensus Guidelines for management of patients with colon and rectal cancer. a personalized approach to clinical decision making. Ann Oncol 23, 2479-2516 
Schmoll HJ, Tabernero J, Maroun J, de Braud F, Price T, van Cutsem E, Hill M, Hoersch S, Rittweger K, Haller DG (2015): Capecitabine plus oxaliplatin compared with fluorouracil/folinicacid as adjuvant therapy for stage III colon cancer: final results of the NO16968 randomized controlled phase III trial. J Clin Oncol $\underline{33}$, 3733-3740

Schmoll HJ, Haustermans K, Price TJ, Nordlinger B, Hofheinz R, Daisne JF, Janssens J, Brenner B, Schmidt $\mathrm{P}$, Reinel $\mathrm{H}$ et al. (2018): Preoperative chemoradiotherapy and postoperative chemotherapy with capecitabine +/- oxaliplatin in locally advanced rectal cancer: final results of PETACC-6. J Clin Oncol $\underline{36}, 3500$

Simmonds PC (2000): Palliative chemotherapy for advanced colorectal cancer: systematic review and metaanalysis. Colorectal Cancer Collaborative Group. BMJ $\underline{321}$, 531-535

Smith NJ, Barbachano Y, Norman AR, Swift RI, Abulafi AM, Brown G (2008): Prognostic significance of magnetic resonance imaging-detected extramural vascular invasion in rectal cancer. Br J Surg 95, 229-236

Smith SR, Swift I, Gompertz H, Baker WN (1988): Abdominoperineal and anterior resection of the rectum with retrocolic omentoplasty and no drainage. Br J Surg $\underline{75}$, 1012-1015

Sobin LH, Gospodarowicz MK, Wittekind C: TNM Classification of Malignant Tumours, 7th ed; Wiley, Somerset 2011

Song C, Chung JH, Kang SB, Kim DW, Oh HK, Lee HS, Kim JW, Lee K-W, Kim JH, Kim JS (2018): Impact of tumor regression grade as a major prognostic factor in locally advanced rectal cancer after neoadjuvant chemoradiotherapy: a proposal for a modified staging system. Cancers (Basel) $\underline{10}, 319$

Sprenger T, Rothe H, Beissbarth T, Conradi L-C, Kauffels A, Homayounfar K, Behnes CL, Rödel C, Liersch T, Ghadimi M (2016): Lymphknotenmetastasen beim ypT1/2-Rektumkarzinom nach neoadjuvanter Radiochemotherapie. Die Achillesferse organerhaltender Operationsverfahren? Chirurg 87, 593-601

Sprenger T, Beißbarth T, Sauer R, Tschmelitsch J, Fietkau R, Liersch T, Hohenberger W, Staib L, Gaedcke J, Raab HR et al. (2018): Long-term prognostic impact of surgical complications in the german rectal cancer trial CAO/ARO/AIO-94. Br J Surg 105, 1510-1518

Starker A, Buttmann-Schweiger N, Krause L, Barnes B, Kraywinkel K, Holmberg C (2018): Krebsfrüherkennungsuntersuchungen in Deutschland: Angebot und Inanspruchnahme. Bundesgesundheitsblatt Gesundheitsforschung Gesundheitsschutz $\underline{61}, 1491-1499$

Statistische Bundesamt (Destatis) (Hrsg): Bevölkerung im Wandel, Annahmen und Ergebnisse der 14. koordinierten Bevölkerungsvorausberechnung; Statistisches Bundesamt, Wiesbaden 2019; https://www.destatis.de/DE/Presse/Pressekonferenzen/2019/Bevoelkerung/pressebroschuerebevoelkerung.pdf?__blob=publicationFile; abgeufen am 12.01.2020

Steliarova-Foucher E, O'Callaghan M, Ferlay J, Masuyer E, Rosso S, Forman D, Bray F, Comber H (2015): The European Cancer Observatory: A new data resource. Eur J Cancer 51, 1131-1143

Stintzing S, Heinemann V, Moosmann N, Hiddemann W, Jung A, Kirchner T (2009): The treatment of colorectal carcinoma with monoclonal antibodies: the importance of KRAS mutation analysis and EGFR status. Dtsch Arztebl Int 106, 202-206

Suzuki T, Sadahiro S, Tanaka A, Okada K, Saito G, Miyakita H, Akiba T, Yamamuro H (2017): A modified classification of prognostic factors based on pathological stage and tumor regression grade in patients with rectal cancer who receive preoperative chemoradiotherapy. Oncology 93, 287-294

Swellengrebel HAM, Bosch SL, Cats A, Vincent AD, Dewit LGH, Verwaal VJ, Nagtegaal ID, Marijnen CAM (2014): Tumour regression grading after chemoradiotherapy for locally advanced rectal cancer: a near pathologic complete response does not translate into good clinical outcome. Radiother Oncol 112, 44-51

Tang PA, Bentzen SM, Chen EX, Siu LL (2007): Surrogate endpoints for median overall survival in metastatic colorectal cancer: literature-based analysis from 39 randomized controlled trials of first-line chemotherapy. $J$ Clin Oncol 25, 4562-4568

Thibodeau SN, Bren G, Schaid D (1993): Microsatellite instability in cancer of the proximal colon. Science 260, 816-819

Toribara NW, Sleisenger MH (1995): Screening for colorectal cancer. N Engl J Med $\underline{332}, 861-867$

Tournigand C, André T, Achille E, Lledo G, Flesh M, Mery-Mignard D, Quinaux E, Couteau C, Buyse M, Ganem G et al. (2004): FOLFIRI followed by FOLFOX6 or the reverse sequence in advanced colorectal cancer: a randomized GERCOR study. J Clin Oncol 22, 229-237 
Trinquart L, Jacot J, Conner SC, Porcher R (2016): Comparison of treatment effects measured by the hazard ratio and by the ratio of restricted mean survival times in oncology randomized controlled trials. J Clin Oncol $\underline{34}, 1813-1819$

Upshaw JN, O'Neill A, Carver JR, Dimond EP, Denlinger CS, Kircher SM, Wagner LI, Ky B, Brell JM (2019): Fluoropyrimidine cardiotoxicity: time for a contemporaneous appraisal. Clin Colorectal Cancer 18, 44-51

Valentini V, van Stiphout RGPM, Lammering G, Gambacorta MA, Barba MC, Bebenek M, Bonnetain F, Bosset JF, Bujko K, Cionini L et al. (2011): Nomograms for predicting local recurrence, distant metastases, and overall survival for patients with locally advanced rectal cancer on the basis of european randomized clinical trials. J Clin Oncol 29, 3163-3172

van Kuilenburg ABP (2004): Dihydropyrimidine dehydrogenase and the efficacy and toxicity of 5-fluorouracil. Eur J Cancer $\underline{40}$, 939-950

van Kuilenburg AB, Vreken P, Abeling NG, Bakker HD, Meinsma R, van Lenthe H, Abreu RA de, Smeitink JA, Kayserili H, Apak MY et al. (1999): Genotype and phenotype in patients with dihydropyrimidine dehydrogenase deficiency. Hum Genet 104, 1-9

van Kuilenburg AB, Haasjes J, Richel DJ, Zoetekouw L, van Lenthe H, Abreu RA de, Maring JG, Vreken P, van Gennip AH (2000): Clinical implications of dihydropyrimidine dehydrogenase (DPD) deficiency in patients with severe 5-fluorouracil-associated toxicity: identification of new mutations in the DPD gene. Clin Cancer Res $\underline{6}, 4705-4712$

Verwaal VJ, Bruin S, Boot H, van Slooten G, van Tinteren H (2008): 8-year follow-up of randomized trial: cytoreduction and hyperthermic intraperitoneal chemotherapy versus systemic chemotherapy in patients with peritoneal carcinomatosis of colorectal cancer. Ann Surg Oncol 15, 2426-2432

Very N, Lefebvre T, El Yazidi-Belkoura I (2018): Drug resistance related to aberrant glycosylation in colorectal cancer. Oncotarget $\underline{9}, 1380-1402$

Vogelstein B, Fearon ER, Hamilton SR, Kern SE, Preisinger AC, Leppert M, Nakamura Y, White R, Smits AM, Bos JL (1988): Genetic alterations during colorectal-tumor development. N Engl J Med 319, 525-532

Vorwerk H, Liersch T, Rothe H, Ghadimi M, Christiansen H, Hess CF, Hermann RM (2009): Gold markers for tumor localization and target volume delineation in radiotherapy for rectal cancer. Strahlenther Onkol $\underline{185}$, 127-133

Walther A, Johnstone E, Swanton C, Midgley R, Tomlinson I, Kerr D (2009): Genetic prognostic and predictive markers in colorectal cancer. Nat Rev Cancer $\underline{9}$, 489-499

Wei X, McLeod HL, McMurrough J, Gonzalez FJ, Fernandez-Salguero P (1996): Molecular basis of the human dihydropyrimidine dehydrogenase deficiency and 5-fluorouracil toxicity. J Clin Invest $\underline{98}, 610-615$

West NP, Finan PJ, Anderin C, Lindholm J, Holm T, Quirke P (2008): Evidence of the oncologic superiority of cylindrical abdominoperineal excision for low rectal cancer. J Clin Oncol 26, $3517-3522$

West NP, Anderin C, Smith KJE, Holm T, Quirke P (2010): Multicentre experience with extralevator abdominoperineal excision for low rectal cancer. Br J Surg $\underline{97}, 588-599$

Wiesing U, Parsa-Parsi RW (2009): Deklaration von Helsinki: Neuste Revision. Deutsches Ärzteblatt $\underline{106}$, 503-506

Wittekind, C. (Hrsg.) (2017): TNM-Klassifikation maligner Tumoren, 8. Auflage; Wiley-VCH Verlag GmbH \& Co. KGaA, Weinheim 2017

Wittekind C, Meyer HJ, (Hrsg.): TNM-Klassifikation maligner Tumoren, 7. Auflage; Wiley-Blackwell, Weinheim 2010

Wittekind, C., Meyer, HJ. (Hrsg.): TNM-Klassifikation maligner Tumoren, 7. Auflage; Wiley-VCH, Weinheim 2013

Wolf M, Zehentmayr F, Belka C (2012): Strahlentherapie des Rektumkarzinoms. Radiologe $\underline{52}, 545-549$

Wong GY, Michalak JC, Sloan JA, Mailliard JA, Nikcevich DA, Novotny PJ, Warner DO, Kutteh L, Dakhil SR, Loprinzi CL (2005): A phase III double blinded, placebo controlled, randomized trial of gabapentin in patients with chemotherapy-induced peripheral neuropathy: a north central cancer treatment group study. J Clin Oncol $\underline{23}, 8001$ 
Wörmann B: Krebsfrüherkennung in Deutschland 2014: Evidenz - aktueller Stand - Perspektiven (Gesundheitspolitische Schriftenreihe der DGHO Band 4), Stand: Mai 2014; Vorstand der DGHO Deutsche Gesellschaft für Hämatologie und Medizinische Onkologie e. V, Berlin 2014;

https://www.dgho.de/publikationen/schriftenreihen/krebsfrueherkennung/140507-

Krebsfrueerkennung_in_Deutschland_2014-WEB-FINAL.pdf; abgerufen am 13.01.2020

Xu Z, Mohile SG, Tejani MA, Becerra AZ, Probst CP, Aquina CT, Hensley BJ, Arsalanizadeh R, Noyes K, Monson JRT et al. (2017): Poor compliance with adjuvant chemotherapy use associated with poorer survival in patients with rectal cancer: an NCDB analysis. Cancer $\underline{123}, 52-61$

Yamashita K, Yada H, Ariyoshi T (2004): Neurotoxic effects of alpha-fluoro-beta-alanine (FBAL) and fluoroacetic acid (FA) on dogs. J Toxicol Sci $\underline{29}, 155-166$

Yothers G, O'Connell MJ, Allegra CJ, Kuebler JP, Colangelo LH, Petrelli NJ, Wolmark N (2011): Oxaliplatin as adjuvant therapy for colon cancer: updated results of NSABP C-07 trial, including survival and subset analyses. J Clin Oncol $\underline{29}$, 3768-3774 


\section{Danksagung}

Zunächst gebührt mein herzlichster und außerordentlicher Dank Herrn Prof. Dr. med. Torsten Liersch. Er hat das Promotionsprojekt konzipiert und durch seine motivierende Art, seine zahlreichen und hilfreichen Anregungen sehr unterstützt, sodass diese Dissertation durch seine stets konstruktive Kritik und freundliche, persönliche Betreuung zum Abschluss gelangen konnte. Ich bedanke mich bei Ihm für das in mich gesetzte Vertrauen.

Ich danke meiner Kobetreuerin Frau PD Dr. med. Friederike Braulke (Klinik für Hämatologie und Medizinische Onkologie der UMG) für Ihre konstruktiven Anregungen und Diskussionen während der beiden Progressreporte.

Dank gilt Herrn Univ.-Prof. Dr. med. Heinz Becker (posthum; ehemaliger Direktor der Klinik für Allgemein-, Viszeral- und Transplantationschirurgie) und Herrn Univ.Prof. Dr. med. Michael Ghadimi (Direktor der Klinik für Allgemein-, Viszeral- und Kinderchirurgie der UMG) für die Möglichkeit, in ihrer Klinik unsere Patienten in den entsprechenden Studien als Prüfärztin zu behandeln, wissenschaftlich zu arbeiten und dieses Promotionsprojekt abschließen zu können.

Bei Herrn Dr. rer nat. Andreas Leha (Institut für medizinische Statistik der UMG) bedanke ich mich für die Beratung und Betreuung bei den multivariaten Analysen der Patientenkohorten.

Herzlich danke ich auch dem Team des Chirurgischen Studiensekretariates mit Frau Elke Stauffer, Frau Susanne Klie, Frau Larissa Geier und Frau Birgit Jünemann für ihren Einsatz während meines Promotionsprojekts in der Patientenbetreuung, Datenerhebung, Asservation von Gewebeproben und für das Korrekturlesen. Ein besonderer Dank geht an Frau Johanna Kreutzer für die Koordination der diesem Projekt zugrunde liegenden Studien und Ihren Einsatz in der finalen Korrektur dieser Arbeit.

Herrn Dirk Weinert danke ich für seinen umfassenden IT-Support 